UNIVERSIDADE DE SÃO PAULO

FACULDADE DE ECONOMIA, ADMINISTRAÇÃO E CONTABILIDADE DEPARTAMENTO DE ADMINISTRAÇÃO PROGRAMA DE PÓS-GRADUÇÃO EM ADMINISTRAÇÃO

\title{
MODELAGEM DE SÉRIES TEMPORAIS FINANCEIRAS MULTIDIMENSIONAIS VIA PROCESSOS ESTOCÁSTICOS E CÓPULAS DE LÉVY
}

\author{
Edson Bastos e Santos \\ Orientador: Prof. Dr. José de Oliveira Siqueira \\ Área de Concentração: Métodos Quantitativos e Informática
}

São Paulo

Dezembro de 2005 
Prof. Dra. Suely Vilela

Reitora da Universidade de São Paulo

Profa. Dra. Maria Tereza Leme Fleury

Diretora da Faculdade de Economia, Administração e Contabilidade

Prof. Dr. Eduardo Pinheiro Gondim de Vasconcellos

Chefe do Departamento de Administração

Prof. Dr. Isak Kuglianskas

Coordenador do Programa de Pós-Graduação em Administração 
UNIVERSIDADE DE SÃO PAULO

FACULDADE DE ECONOMIA, ADMINISTRAÇÃO E CONTABILIDADE DEPARTAMENTO DE ADMINISTRAÇÃO

PROGRAMA DE PÓS-GRADUÇÃO EM ADMINISTRAÇÃO

\title{
MODELAGEM DE SÉRIES TEMPORAIS FINANCEIRAS MULTIDIMENSIONAIS VIA PROCESSOS ESTOCÁSTICOS E CÓPULAS DE LÉVY
}

\author{
Edson Bastos e Santos \\ Orientador: Prof. Dr. José de Oliveira Siqueira \\ Área de Concentração: Métodos Quantitativos e Informática
}

Dissertação apresentada ao Departamento de Administração da Faculdade de Economia, Administração e Contabilidade da Universidade de São Paulo como requisito parcial para a obtenção do título de Mestre em Administração.

São Paulo

Dezembro de 2005 
@ 2005 Edson Bastos e Santos

Texto elaborado em ${ }^{\mathrm{A}} \mathrm{T}_{\mathrm{E}} \mathrm{X}$ via interface Scientific Workplace 5.0.

Quadros e figuras elaborados em Mathematica 5.1. 


\section{MODELAGEM DE SÉRIES TEMPORAIS \\ FINANCEIRAS MULTIDIMENSIONAIS \\ VIA PROCESSOS ESTOCÁSTICOS \\ E CÓPULAS DE LÉVY}

por

Edson Bastos e Santos

Dissertação aprovada, como requisito parcial para a obtenção do título de Mestre em Administração, pelo Departamento de Administração da Faculdade de Economia, Administração e Contabilidade da Universidade de São Paulo, pela Comissão Examinadora formada pelos docentes:

Prof. Dr. José de Oliveira Siqueira

Doutor pela Universidade de São Paulo

Professor do Departamento de Administração da FEA/USP

Presidente da Comissão Examinadora

Prof. Dr. Nelson Ithiro Tanaka

Doutor pela Cornell University

Professor do Departamento de Estatística do IME/USP

Dr. Christian Johannes Zimmer

Doutor pela Universidade de São Paulo

Membro da Superintendência de Análise Quantitativa do Banco Itaú 

A minha amada esposa que sempre apoiou meus sonhos e, por me fazer feliz, faz de mim um ser humano melhor. 



\section{Agradecimentos}

À Deus, por ter me revelado os caminhos e me guiado sempre para o Bem.

A minha amada esposa Lucia, pela motivação constante em me apoiar nos momentos mais desafiadores deste trabalho, por acreditar nos meus esforços e por retribuir com tanta dedicação e carinho todas as ausências que tive em nome deste projeto.

Ao meu orientador Prof. Dr. Siqueira, por ter me aceito quando eu ainda nada poderia provar, pela confiança que depositou em mim, pelo otimismo e respeito com que tratou as minhas idéias e, pela sua amizade e compreensão durante todos os momentos.

Aos docentes da USP, principalmente aos que participaram da Comissão de Exame de Qualificação, Profs. Drs. Canton e Alessandra, aos que participaram da Comissão Examinadora, Profs. Drs. Tanaka e Zimmer, que apresentaram recomendações pertinentes relativas a este estudo e, ao Prof. Dr. Securato que me motivou a iniciar os meus estudos na Pós-graduação.

Aos meus queridos pais Roberto e Neusa, que sempre incentivaram os meus estudos, ofereceram todas as oportunidades de ensino que estavam ao alcance e contribuíram para a pessoa que sou hoje. Devo aos meus pais o exemplo familiar de dedicação e trabalho em prol da academia.

Ao meu querido avô Elon, cuja vontade de viver, generosidade e sabedoria colhidas nestes mais de oitenta anos, servem de exemplo para a minha vida.

Aos meus sogros Sebastião e Edelazil, que acompanharam as minhas pesquisas e souberam entender a minha dedicação aos estudos.

A minha irmã Taís e seu noivo Marco, a minha irmã Simone e meu cunhado Walter e aos meus sobrinhos Isabella e Giancarlo, cujo convívio familiar acalentou e sublimou tantos dos meus esforços.

Ao Banco Central do Brasil, por ter permitido e incentivado este projeto, em especial, aos meus estimados colegas, Agnaldo, Andréa, Armando, Carlos, Cláudio, Hilton, Simão e Walter, cuja compreensão e amizade em muito me ajudaram nestes últimos anos.

Aos meus colegas de Pós-graduação Carlos, Daniel, Danilo, Fábio, Henrique, Marco, Mauri e Paiva, companheiros de disciplinas e de importantes discussões, que acompanharam e auxiliaram no desenvolvimento do tema pesquisado.

À Lícia do Departamento de Métodos Quantitativos e Informática, Elaine do COMUT (Programa de Comutação Bibliográfica), à Margarida e Dulcinéia do 
SBD/FEA (Serviço de Biblioteca e Documentação da FEA), à Valéria da Secretaria de Pós-graduação da FEA, à Eloisa do PPGA (Programa de Pós-graduação em Administração da FEA), à Giseli da Seção de Processamento Técnico do SBD/FEA, à Leila do CCE (Centro de Computação Eletrônica), ao Francisco do LCCA (Laboratório de Computação Científica Avançada), às bibliotecárias do IME (Instituto de Matemática e Estatística) e da POLI (Escola Politécnica) por terem se disponibilizado a me informar e assessorar ao longo dos meus estudos. 
"Though this be madness, yet there is method in it."

Willian Shakespeare (1564-1616)

"It is a truth very certain that when it is not in our power to determine what is true we ought to follow what is most probable."

René Descartes (1596-1650)

"If I have seen further it is by standing upon the shoulders of giants."

Isaac Newton (1643-1727)

"The art of discovering the causes of phenomena, or true hypothesis, is like the art of decyphering, in which an ingenious conjecture greatly shortens the road."

Gottfreid Wilhelm Von Leibniz (1646-1716)

"Science is the great antidote to the poison of enthusiasm and superstition."

Adam Smith (1723-1790)

"Such is the advantage of a well constructed language that its simplified notation often becomes the source of profound theories."

Pierre-Simon de Laplace (1749-1827)

"A mathematician who is not also something of a poet will never be a complete mathematician." Karl Theodor Wilhelm Weierstrass (1815-1897)

"A theory is something nobody believes, except the person who made it. An experiment is something everybody believes, except the person who made it."

Albert Einstein (1879-1955)

"A scientist worthy of his name, above all a mathematician, experiences in his work the same impression as an artist; his pleasure is as great and of the same nature."

Jules Henri Poincaré (1854-1912)

"The traveler often has the choice between clibing a peak or using a cable car."

William Feller (1906-1970) 



\section{Resumo}

O principal objetivo deste estudo é descrever modelos para séries temporais de ativos financeiros que sejam robustos às tradicionais hipóteses: distribuição gaussiana e continuidade. O primeiro capítulo está preocupado em apresentar, de uma maneira geral, os conceitos matemáticos mais importantes relacionadas a processos estocásticos e difusões. O segundo capítulo trata de processos de incrementos independentes e estacionários, i.e., processos de Lévy, suas trajetórias estocásticas, propriedades distribucionais e, a relação entre processos markovianos e martingales. Alguns dos resultados apresentados neste capítulo são a estrutura e as propriedades dos processos compostos de Poisson, medida de Lévy, decomposição de Lévy-Itô e representação de Lévy-Khinchin. O terceiro capítulo mostra como construir processos de Lévy por meio de transformações lineares, inclinação da medida de Lévy e subordinação. Uma atenção especial é dada aos processos subordinados, tais como os modelos variância gama, normal gaussiana invertida e hiperbólico generalizado. Neste capítulo também é apresentado um exemplo pragmático com dados brasileiros de estimação de parâmetros por meio do método de máxima verossimilhança. O quarto capítulo é devotado aos modelos multidimensionais e, introduz os conceitos de cópulas ordinária e de Lévy. Mostra-se que é possível caracterizar a dependência entre os componentes de um processo de Lévy multidimensional por meio da cópula de Lévy. Entre os resultados apresentados estão as generalizações do teorema de Sklar e a família de cópulas de Arquimedes aos processos de Lévy. Este capítulo também apresenta alguns exemplos que utilizam métodos de Monte Carlo, para simular processos de Lévy bidimensionais.

Palavras-Chave: análise de séries temporais, processos estocásticos, processos de difusão, processos de Lévy, processos de Poisson, processos descontínuos, processos não gaussianos estáveis, subordinação, risco, movimento browniano, cópulas, distribuições hiperbólicas, mercados incompletos. 



\section{Abstract}

The main objective of this study is to describe models for financial assets time series that are robust to the traditional hypothesis: gaussian distributed and continuity. The first chapter are devoted to introduce the most important mathematical tools related to difusions and stochastic processes in general. The second chapter is concerned in the study of independent and stationary increments, i.e., Lévy processes, their sample paths behavior, distributional properties, and the relation to Markov and martingales processes. Some of the results presented are the structure and properties of a compound Poisson processes, Lévy measure, Lévy-Itô decomposition and Lévy-Khinchin representation. The third chapter demonstrates how to construct Lévy processes via linear transformation, tempering the Lévy measure and subordination. A special attention is given to several types of subordinated processes, comprising the variance gamma, the normal inverse gaussian and the generalized hyperbolic models. An pragmatic example of parameter estimation for brazilian data using the method of maximum likelihood is also given. Chapter four is devoted to multidimensional models, which introduces the notion of ordinary and Lévy copulas. It is shown that modelling via Lévy copula it is possible to characterize the dependence among components of multidimensional Lévy processes. Some of the results presented are generalizations of the Sklar's theorem and the Archmedian family of copulas for Lévy processes. This chapter also presents some examples using Monte Carlo methods for simulating bidimensional Lévy processes.

Keywords: time series analysis, stochastic processes, difusion processes, Lévy processes, Poisson processes, discontinuous processes, non-gaussian stable processes, subordination, risk, brownian motion, copulas, hyperbolic distributions, incomplete makets. 



\section{Sumário}

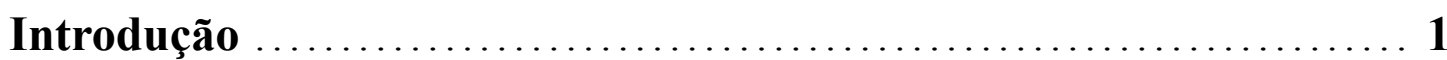

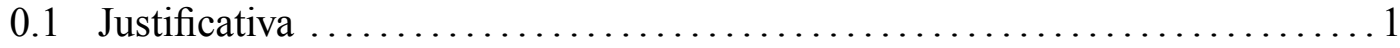

0.1 .1 Modelos e Fatos Estilizados ...........................6 6

0.1 .2 Hedge e Gerenciamento de Riscos ...................... 8

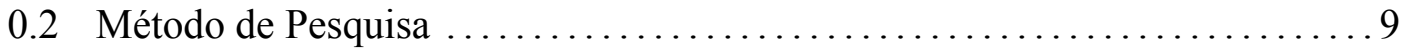

0.3 Revisão Bibliográfica .................................... 10

1 Processos e Medidas Estocásticas .......................... 13

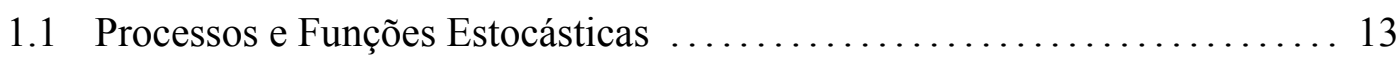

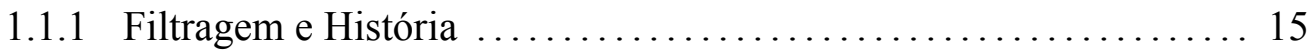

1.1.2 Tempo Estocástico .............................. 17

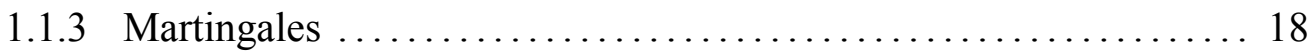

1.1.4 Processo Previsível ................................... 20

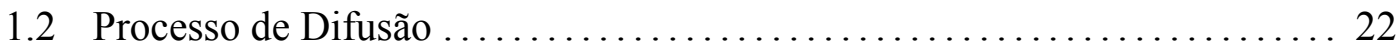

1.2.1 Propriedades Markovianas ......................... 22

1.2.2 Movimento Browniano ................................... 23

1.2.3 Processo de Difusão de Itô ........................... 24

1.3 Processo de Poisson ....................................... 26

1.3.1 Variáveis Estocásticas Exponenciais .................. 26

1.3.2 A Distribuição de Poisson............................ 29

1.3.3 Propriedades do Processo de Poisson .................... 30

1.3.4 Processo de Poisson Compensado ....................... 36

1.3.5 Processo de Contagem ............................ 37

1.4 Medidas Estocásticas e Processos Pontuais ....................... 40

1.4.1 Medida Estocástica de Poisson ou Processos Pontuais .......... 41

1.4.2 Medida Estocástica Compensada de Poisson ................ 44

1.4.3 Construindo Processos com Saltos .................... 44

1.4.4 Processo Pontual Registrado ....................... 46

2 Processos Estocásticos de Lévy ........................ 50

2.1 Processo Composto de Poisson ......................... 53

2.2 Medida de Salto do Processo Composto de Poisson ................ 59

2.3 Atividade Infinita do Processo de Lévy ....................... 64

2.4 Trajetórias de um Processo de Lévy $\ldots \ldots \ldots \ldots \ldots \ldots \ldots \ldots \ldots \ldots \ldots \ldots \ldots \ldots \ldots \ldots \ldots \ldots$ 


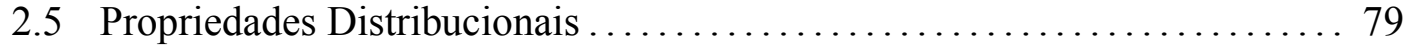

2.6 Distribuições e Processos Estáveis $\ldots \ldots \ldots \ldots \ldots \ldots \ldots \ldots \ldots \ldots \ldots \ldots \ldots . \ldots \ldots$

2.7 Processos de Lévy e Markov ............................. 86

2.8 Martingales e Processos de Lévy . .......................... 89

3 Modelagem Unidimensional de Processos Estocásticos de Lévy .. 91

3.1 Salto-Difusão versus Processo de Lévy de Atividade Infinita........... 91

3.2 Transformando os Processos de Lévy ....................... 93

3.2.1 Transformação Linear . ........................... 93

3.2.2 Subordinação.................................. 96

3.2.3 Inclinando e Temperando a Medida de Lévy ................ 99

3.3 Modelos do Tipo Salto-Difusão $\ldots \ldots \ldots \ldots \ldots \ldots \ldots \ldots \ldots \ldots \ldots \ldots \ldots \ldots \ldots \ldots$

3.4 Processos de Lévy via Subordinação Browniana .................. 101

3.4.1 Subordinação do Movimento Browniano.................. 105

3.4.2 Modelos Baseados na Subordinação Browniana .............. 107

3.5 Processo Estável Temperado ............................... 109

3.6 Modelo Hiperbólico Generalizado .......................... 117

\section{Modelagem Multidimensional de Processos Estocásticos de}

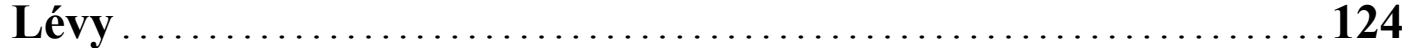

4.1 Modelagem via Subordinação Browniana ...................... 126

4.2 Modelagem via Processos Compostos de Poisson .................... 129

4.3 Cópulas para Variáveis Estocásticas .......................... 131

4.3.1 Exemplos de Cópulas .................................. 135

4.3.2 Definições Estendidas aos Casos Multivariados ............... 138

4.4 Conceitos de Dependência para Processos de Lévy ................... 139

4.5 Cópulas para Processos de Lévy com Saltos Positivos . . . . . . . . . . . . . 142

4.5.1 Integral Caudal e Medidas de Lévy ...................... 145

4.5.2 Exemplos de Cópulas de Lévy Positivas ................... 147

4.5.3 Dependência de Processos Compostos de Poisson ............. 150

4.5.4 Construção de Cópulas Positivas de Lévy .................. 152

4.5.5 Interpretação Probabilística ........................... 153

4.5.6 Definições Estendidas aos Casos Multivariados .............. 154

4.6 Cópulas de Processos de Lévy Generalizados .................... 155

4.6.1 Medidas de Lévy com Densidades ..................... 156 
4.6.2 Cópulas de Lévy Generalizada $\ldots \ldots \ldots \ldots \ldots \ldots \ldots \ldots \ldots \ldots \ldots$

4.6.3 Construção de Cópulas de Lévy ....................... 160

4.6.4 Definições Estendidas aos Casos Multivariados .............. 161

4.7 Construindo Modelos Multivariados por Meio de Cópulas de Lévy..... . 162

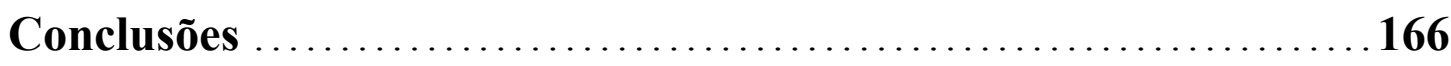

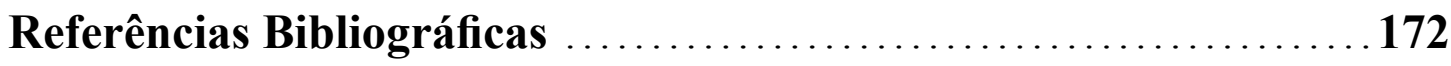

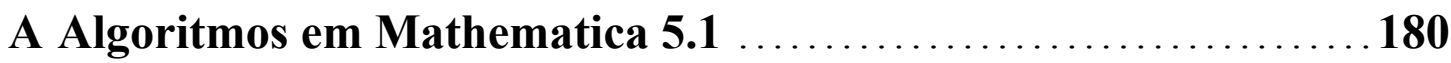

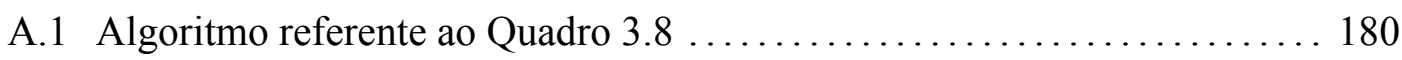

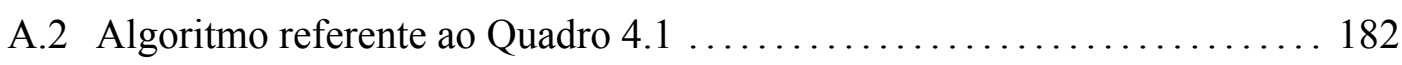

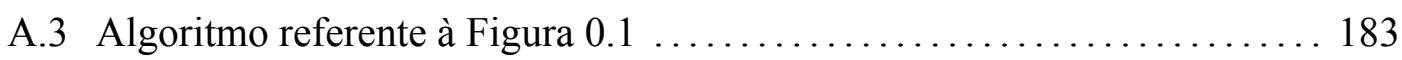

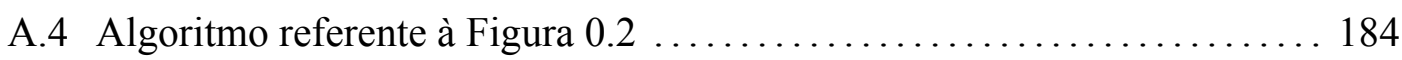

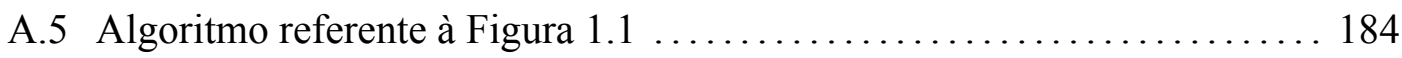

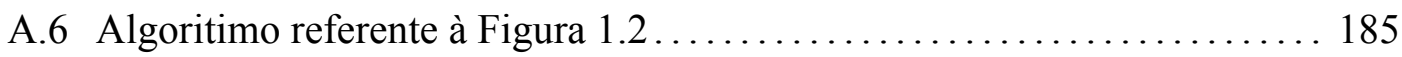

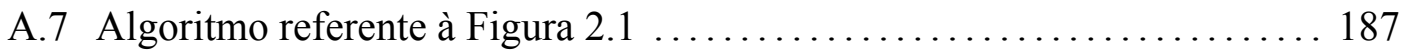

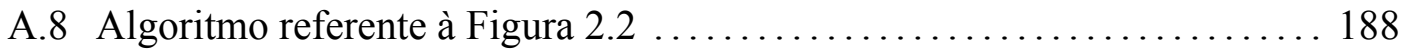

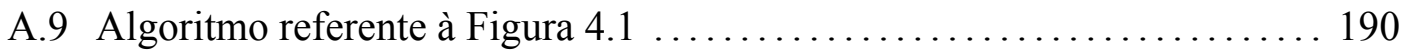

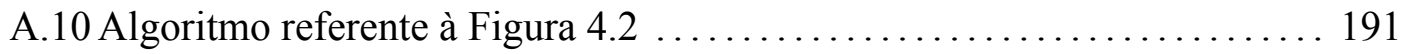

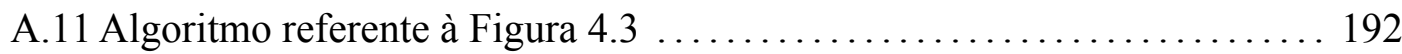

B Teoria da Medida e Integração ............................ 194

B.1 $\sigma$-álgebra e Medida....................................... 194

B.2 Medidas e Funções: Integração $\ldots \ldots \ldots \ldots \ldots \ldots \ldots \ldots \ldots \ldots \ldots \ldots \ldots \ldots \ldots$

B.3 Continuidade Absoluta e Densidade ........................... 200

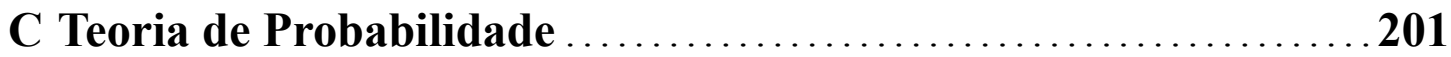

C.1 Variável Estocástica e Espaço Probabilístico ...................... 201

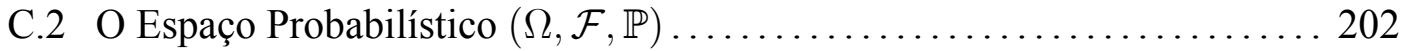

C.3 Função Característica .................................... 204

C.4 Função Geratriz de Momentos ........................... 206

C.5 Função Geratriz de Cumulantes .............................. 207

D Convergência de Variáveis Estocásticas ................... 209

D.1 Convergência Quase-Certa .................................. 209

D.2 Convergência em Probabilidade .......................... 210 
D.3 Convergência em Distribuição . ................................ 210

E Funções Bessel Modificadas ........................... 213

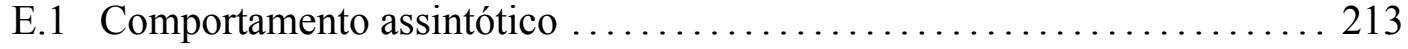

E.2 Expressões analíticas de funções Bessel modificadas definidas nos

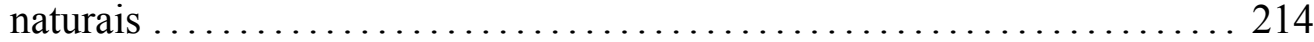

E.3 Representação por integral .............................. 214

E.4 Relação entre as funções Bessel e o argumento real ............... 215 


\section{Lista de Acrônimos}

BSM Modelo de Black-Scholes-Merton

c.g.f. função geratriz de cumulantes (cumulant generating function)

e.g. $\quad$ por exemplo (exempli gratia)

et al. e outros(as) [et alii (masc.), et aliae (fem.) ou et alia (neutro)]

GIG gaussiana invertida generalizada (generalized inverse gaussian)

GH hiperbólica generalizada (generalized hyperbolic)

g.l. graus de liberdade

Ibovespa Índice da Bolsa de Valores de São Paulo

i.d. independentemente distribuída

i.e. isto é (id est)

i.i.d. identicamente e independentemente distribuída

$\log$-retorno $\ln \left(S_{t} / S_{t-\delta}\right)$, para $\delta>0$

m.g.f. função geratriz de momentos (moment generating function)

MB movimento browniano

MBG movimento browniano geométrico

NIG normal gaussiana invertida (normal inverse gaussian)

S.l. $\quad$ local indeterminado (Sine loco)

t.d. tendência determinística (drift)

p.g.d. processo gerador de dados

q.c. quase-certa (almost sure)

q.l. quase em todos os locais (almost everywhere)

Q.E.D. como quer-se-ia demonstrar (Quod Erat Demonstrandum)

v.e. variável estocástica

VG variância gama (variance gamma) 



\section{Lista de Símbolos}

$\mathbb{R}^{d} \quad$ Espaço Euclidiano de dimensão $d ; \mathbb{R}^{1} \triangleq \mathbb{R}$.

$\overline{\mathbb{R}}^{d} \quad$ Espaço Euclidiano estendido de dimensão $d . \overline{\mathbb{R}}^{1} \triangleq \mathbb{R} \cup\{-\infty\} \cup\{+\infty\}$.

$\mathbb{R}_{+} \quad$ Numeros reais não negativos.

$\sigma(\mathcal{A}) \quad$ A menor $\sigma$-algebra mensurável em relação a $\mathcal{A}$. $\mathcal{A}$ pode ser uma coleção de subconjuntos ou uma coleção de funções.

$\# A \quad$ Numero de elemento do conjunto $A$.

$\mathbb{P}(A) \quad$ Probabilidade de ocorrência do evento $A$.

$\mathbb{E}[X] \quad$ Esperança da v.e. $X$.

$\mathbb{E}[X \mid Y] \quad$ Esperança da v.e. $X$ condicionada a v.e. $Y$.

$\mathbb{V}[X] \quad$ Variância da v.e. $X$.

$\mathbb{C}[X ; Y] \quad$ Covariância entre a v.e. $X$ e a v.e. $Y$.

$\Phi_{X} \quad$ Função característica da v.e. $X$.

$\Psi_{X} \quad$ Função geratriz de cumulantes de $X$.

$\phi_{t}(\cdot) \quad$ Função característica de um processo de Lévy.

$\psi(\cdot) \quad$ Expoente característico de um processo de Lévy.

$M_{X} \quad$ Função geratriz de momentos de $X$.

$J_{X} \quad$ Medida de salto de um processo cadlag $\left\{X_{t}\right\}_{t \geq 0}$.

$X \stackrel{d}{=} Y \quad X$ e $Y$ possuem a mesma distribuição.

$X_{n} \stackrel{d}{\longrightarrow} X\left\{X_{n}\right\}$ converge em distribuição para $X$.

$\mu_{n} \Longrightarrow \mu \quad\left\{\mu_{n}\right\}$ converge fracamente para $\mu$.

$X_{n} \stackrel{\mathbb{P}}{\longrightarrow} X\left\{X_{n}\right\}$ converge em probabilidade para $X$.

$\mathcal{B}(E) \quad \sigma$-algebra de Borel do conjunto $E$.

$\omega \in \Omega \quad$ Cenários estocástico.

$\left\{B_{t}\right\}_{t \geq 0} \quad$ Movimento Browniano.

$\left\{W_{t}\right\}_{t \geq 0} \quad$ Processo de Wiener.

$a . b \quad$ Produto escalar entre $a$ e $b$.

$\mathcal{D}(f) \quad$ Domínio da função $f$.

$\mathcal{I}(f) \quad$ Imagem da função $f$. 
$x \rightarrow f(x) \quad f$ é função de $x$.

$f: A \mapsto B$ O $\mathcal{D}(f)=A$ mapeia $\mathcal{I}(f)=B$.

$a \approx b \quad a$ é aproximadamente igual a $a$.

$\mathbb{P} \sim \mathbb{Q} \quad$ A medida de probabilidade $\mathbb{P}$ é equivalente a medida de probabilidade $\mathbb{Q}$.

$f \sim g \quad$ A função $f$ comporta-se como a função $g$.

$X \stackrel{d}{=} \mathcal{N}(\cdot)$ A variável estocástica $X$ possui distribuição gaussiana.

$a \downarrow b \quad a$ se aproxima de $b$ pela direita.

$a \uparrow b \quad a$ se aproxima de $b$ pela equerda.

$a \triangleq b \quad a$ é definido como $b$.

$a \wedge b \quad \min \{a ; b\}$.

$a \vee b \quad \max \{a ; b\}$.

$\mathcal{N}(\alpha ; \beta) \quad$ Distribuição gaussiana com esperança $\alpha$ e variância $\beta$.

$\mathcal{C}^{n} \quad$ Função diferenciável até a ordem $n$.

$a^{T} \quad$ Transposta do vetor coluna $a$.

$|x| \quad$ Norma do vetor $x:|x| \triangleq \sqrt{x^{T} x}$.

sup Supremo (menor limite superior).

inf Ínfimo (maior limite inferior).

$\operatorname{Re}\{x\} \quad$ Argumento real do número $x$.

$\Gamma[\cdot] \quad$ Função gama.

$\exp \{\cdot\} \quad$ Exponencial.

$\ln \{\cdot\} \quad$ logaritmo natural. 


\section{Lista de Quadros}

Quadro 0.1 Modelando os movimentos de mercado: modelos de

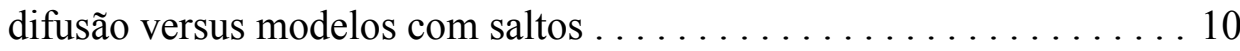

Quadro 2.1 Exemplos de Distribuições e Funções Características . . . . . . . . . 74

Quadro 3.1 Comparação da modelagem por meio de processos compostos de Poisson versus de atividade infinita. . . . . . . . . 92

Quadro 3.2 Dois modelos de salto-difusão: Merton e Kou. . . . . . . . . . . . . 101

Quadro 3.3 Subordinador Gama versus Subordinador Gaussiana Invertida . . . . . 106

Quadro 3.4 Dois modelos baseados na subordinação browniana: processo variância gama e processo normal invertido gaussiano. ................................. 109

Quadro 3.5 Propriedades do modelo hiperbólico generalizado. . . . . . . . . . . . 120

Quadro 3.6 Casos particulares da distribuição hiperbólica generalizada. . . . . . . 121

Quadro 3.7 Características das séries temporais de 11 ativos financeiros

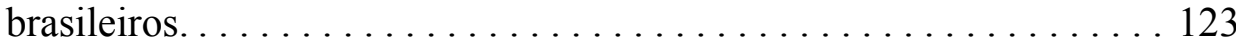

Quadro 3.8 Estimação dos parâmetros de 11 ativos financeiros brasileiros pelo método de máxima verossimilhança para a classe de distribuições hiperbólicas $(\lambda=1)$. A esquerda o resultado ótimo da função de verssimilhança $\mathcal{L}$ dado. $\mathrm{E}$ as

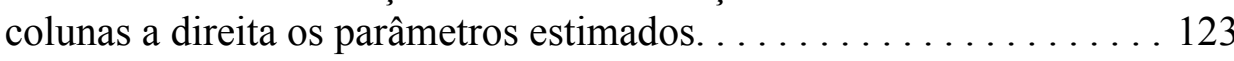

Quadro 4.1 Independência versus decorrelação: processos independentes não podem ser modelados por meio de MB multidimensional utilizando um subordinador unidimensional. 



\section{Lista de Figuras}

Figura 0.1 Ibovespa (curva preta) versus simulação do MBG (curva vermelha) no período de 01.01 .2000 até $01.01 .2005 \ldots \ldots \ldots \ldots \ldots 2$

Figura 0.2 Série intra-diária do Ibovespa em intervalos de 1 minuto no período de $22.07 .2002(10: 00 h)$ até $18.09 .2002(17: 13 \mathrm{~h}) \ldots \ldots \ldots \ldots 3$

Figura 1.1 Simulação de dois processos de Poisson pelo mesmo p.g.d.

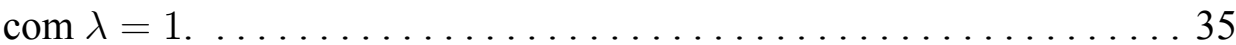

Figura 1.2 Processos de Poisson simulados na Figura 1.1 sem a tendência determinística........................... 37

Figura 2.1 Duas trajetórias de um processo composto de Poisson, com tamanho dos saltos distribuídos por $\mathcal{N}(0 ; 1) \ldots \ldots \ldots \ldots \ldots \ldots 4$

Figura 2.2 A figura a acima mostra uma trajetória típica de um movimento browniano com tendência determinística e, a figura abaixo mostra uma trajetória típica de um processo de salto-difusão.

Figura 2.3 A relação entre processos de Lévy, processos auto-similares e processos gaussianos.

Figura 3.1 Histograma e p.d.f. da distribuição gaussiana e hiperbólica com parâmetros obtidos pelo método de máxima verossimilhança de 11 ativos financeiros brasileiros.

Figura 4.1 Simulação de Monte Carlo de duas marginais normais padronizadas com correlação igual a $\rho=0,8$. Direita superior: simulação da cópula de Clayton. Esquerda superior: simulação da cópula gaussiana. Direita e esquerda inferior quantis $99 \%, 95 \%, 90 \%, 75 \%, 50 \%$ e $25 \%$ das cópulas acima.

Figura 4.2 Trajetória de um processo variância gama bidimensional com estruturas de dependência diferentes para os movimentos positivos e negativos. 164

Figura 4.3 Trajetórias de dois processos variância gama simétricos sendo os saltos positivos copulados com os negativos e vice-versa. 



\section{Introdução}

\subsection{Justificativa}

Na modelagem das variações nos preços por meio de processos estocásticos, o movimento browniano é, sem dúvida, o instrumento mais utilizado. O movimento browniano é um processo estocástico $B=\left\{B_{t}\right\}_{t \geq 0}$ com incrementos independentes e estacionários que seguem uma distribuição gaussiana. O movimento browniano (MB) é, certamente, a origem da análise estocástica de hoje [Definição 1.10].

A modelagem em finanças e o MB já caminham em conjunto desde o século passado, quando Bachelier (1900) propôs e Einstein (1905) formulou o MB, o preço de um ativo financeiro $S_{t}$ foi modelado da seguinte maneira:

$$
S_{t}=s_{0}+\sigma W_{t}
$$

sendo que $W=\left\{W_{t}\right\}_{t \geq 0}$ é um processo de Wiener (caso particular de um MB). Uma versão multiplicativa do modelo proposto por Bachalier permitiu o desenvolvimento do modelo desenvolvido por Black \& Scholes (1973) e Merton (1973), sendo que a variação relativa do preço $S_{t}$ segue um movimento browniano geométrico (MBG):

$$
S_{t}=s_{0} \exp \left(\mu t+\sigma W_{t}\right)
$$

que em forma diferencial é

$$
\frac{d S_{t}}{S_{t}}=\left(\mu+\frac{\sigma^{2}}{2}\right) d t+\sigma d W_{t} .
$$

A relação dada entre $d S_{t} / S_{t}$ e $d \ln S_{t}$ é apresentada por Siqueira (1999), p.150:

$$
\frac{d S_{t}}{S_{t}}=d \ln S_{t}+\frac{\sigma^{2}}{2} \text {. }
$$

A Figura 0.1 representa duas curvas: a evolução do Ibovespa durante o período de 01.01.2000 até 01.01.2005 (fonte dos dados: Reuters), e um caminho observado de um MB, com a mesma tendência determinística (t.d.) e volatilidade do Ibovespa. A primeira vista, não é possível distinguir entre um e outro, pois a evolução do Ibovespa aparentemente é igual ao MB. 


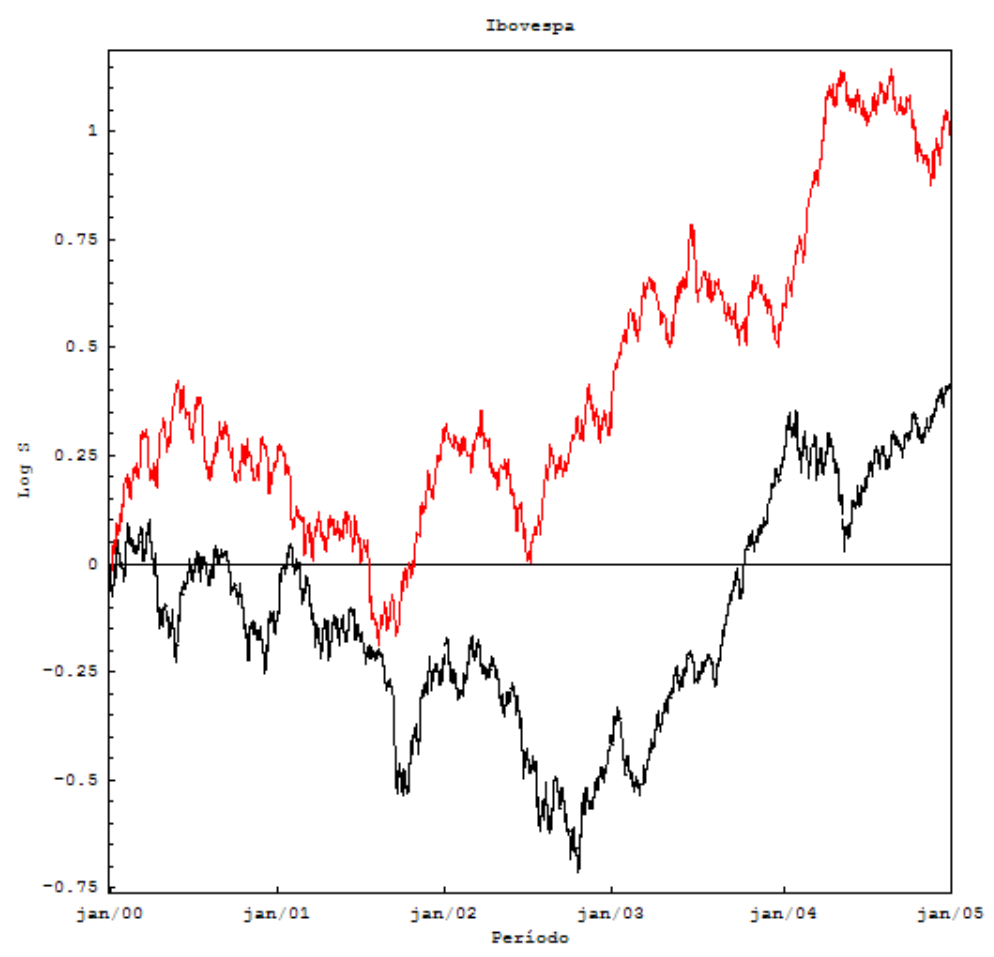

Figura 0.1. Ibovespa (curva preta) versus simulação do MBG (curva vermelha) no período de 01.01.2000 até 01.01.2005. 


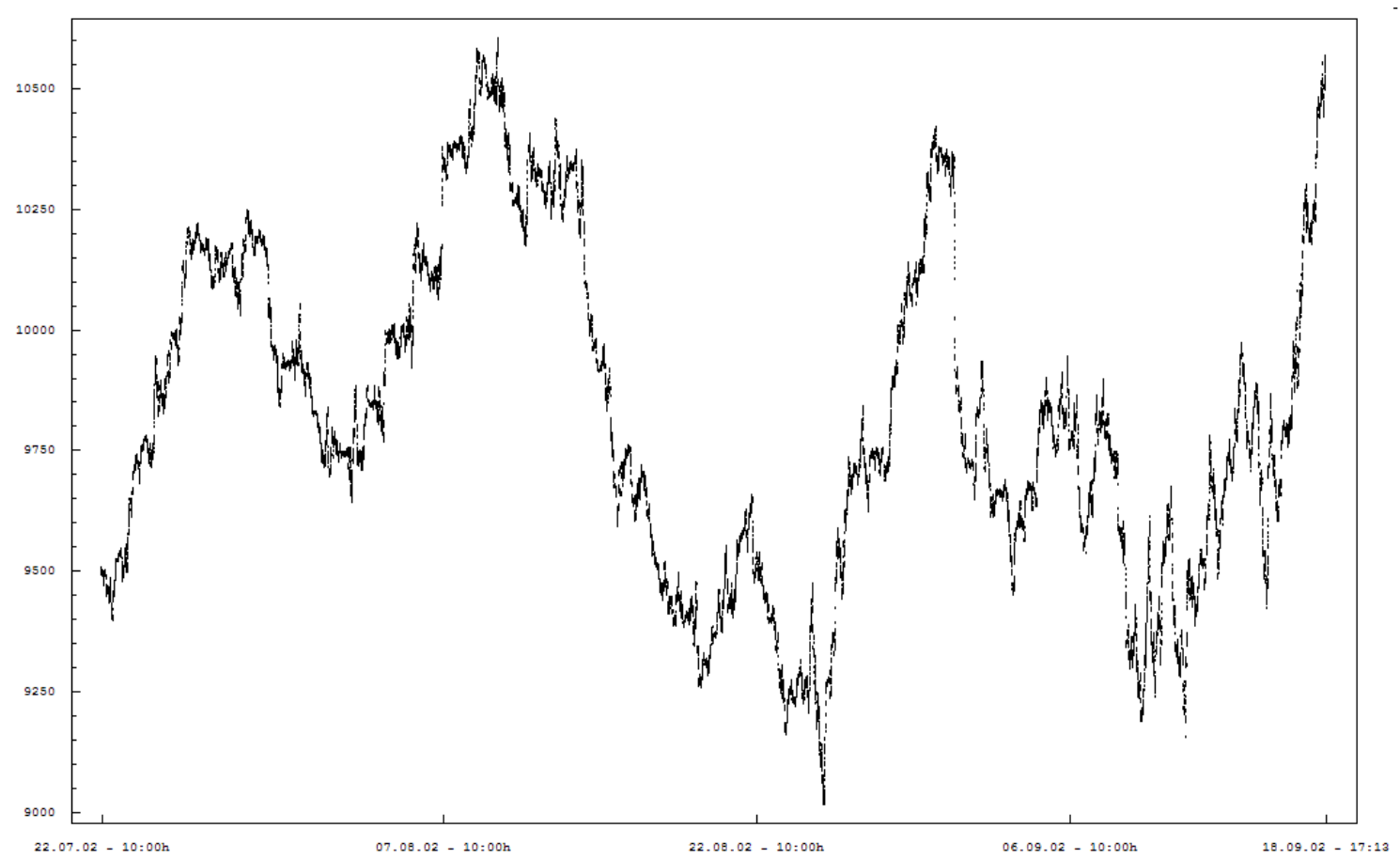

Figura 0.2. Série intra-diária do Ibovespa em intervalos de 1 minuto no período de 22.07.2002 (10:00h) até 18.09.2002 (17:13h).

Uma propriedade importante do $\mathrm{MB}$ é que as trajetórias estocásticas são contínuas, i.e., um caminho estocástico é uma função contínua do tempo $t \longmapsto B_{t}$. Cabe ressaltar que, esta propriedade já é suficiente para diferenciar um MB da evolução do Ibovespa, pois o Ibovespa move-se por saltos, que implicam na existência de descontinuidades na trajetória observada do índice. A Figura 0.2 apresenta a série intra-diária do Ibovespa. O Ibovespa, como pode ser observado, não apresenta uma propriedade importante do MB, que é a invariância de escala, ou seja, as propriedades estatísticas do Ibovespa parecem ser distintas para escalas diferentes de tempo adotadas. Ao reduzir a escala da evolução do Ibovespa torna-se mais fácil identificar as descontinuidades do processo e verificar que as propriedades do processo que orienta o Ibovespa distanciamse bastante das propriedades do MB. No caso de escalas intra-diárias, o que se observa é que o Ibovespa se move essencialmente por saltos.

Assim, o MB não consegue distinguir diferenças de escala no comportamento do preço. Visto que os preços se movem essencialmente por saltos em escalas intra-diárias, em escalas mensais, também se verificará a presença de saltos, enquanto que apenas em 
escalas relativamente grandes, como anos, os preços se comportarão de forma semelhante ao MB. Apesar do modelo Black-Scholes-Merton (BSM) ser adaptável à escala de tempo desejada, ele não se comporta adequadamente em relação à propriedade de agregação do tempo, i.e., mudanças de escalas de tempo. Assim, é difícil modelar igualmente o comportamento dos log-retornos de ativos financeiros em escalas de tempo distintas, que podem variar de poucos minutos até vários anos, o que significa que é crucial especificar a escala adequada para as aplicações do modelo. A perspectiva deste estudo é buscar entender como modelar adequadamente o comportamto dos preços através de processos de Lévy, e estimar os parâmetros do processo gerador de dados (p.g.d.) deste processo para ativos financeiros, e quando possível de ativos financeiros brasileiros.

É claro, que o BSM não é o único modelo construído através de movimentos brownianos. Existem modelos baseados em difusões de Markov não-lineares, cuja volatilidade instantânea depende do preço e do tempo, por meio de uma função de volatilidade local, como foi proposto por Dupire (1994) e Derman \& Kani (1994):

$$
\frac{d S_{t}}{S_{t}}=\sigma\left(t, S_{t}\right) d W_{t}+\nu d t
$$

sendo que $\nu=\mu+\frac{1}{2} \sigma^{2}$. Outra possibilidade de modelo estocástico foi desenvolvido por Hull \& White (1987), no qual o preço $S_{t}$ é um componente de uma difusão bivariada $\left(S_{t}, \sigma_{t}\right)$ orientado por um MB bidimensional $\left(W_{t}^{1}, W_{t}^{2}\right)$, sendo que $W_{t}^{1}$ e $W_{t}^{2}$ não são necessariamente independentes:

$$
\begin{aligned}
\frac{d S_{t}}{S_{t}} & =\sigma_{t} d W_{t}^{1}+\mu d t, \\
\sigma_{t} & =f\left(Y_{t}\right) \\
d Y_{t} & =\alpha_{t} d t+\gamma_{t} d W_{t}^{2} .
\end{aligned}
$$

Enquanto estes modelos parecem ter propriedades estatísticas mais flexíveis, eles compartilham a propriedade de continuidade do $\mathrm{MB}$, que não é compatível com as observações reais dos preços em escalas de tempo diferentes. Ao assumir que os preços movem-se de maneira contínua implica negligenciar os movimentos abruptos nos quais estão concentrados a maior parte do risco econômico destes ativos.

Dado que a continuidade do processo realiza um papel importante na determinação das propriedades dos modelos de processos de difusão [propriedade 1 da 
Definição 1.8; Definição B.3], é de interesse saber se os resultados obtidos por estes modelos são robustos ao excluir a hipótese de continuidade.

Este estudo, inicialmente, buscará relacionar a precificação de opções com a modelagem de processos descontínuos, e justificar, desta maneira, a importância do conceito por meio de exemplos em finanças, para que as definições e proposições apresentadas sejam melhor entendidas.

Os modelos que serão propostos neste estudo, nos quais os preços comportam-se de maneira descontínua, são invariantes para todas as escalas de tempo de interesse, o que demonstra a importância do estudo. Ao examinar os principais assuntos que vêm sendo estudados pela área de finanças quantitativas em relação os modelos com saltos, é possível identificar que os resultados dos modelos de difusão não são robustos à exclusão da hipótese de continuidade no processo dos preços. Assim é necessário considerar modelos que permitam a inclusão de saltos no processo do preço.

Uma abordagem comum para promover a utilização de modelos com saltos é comparar estes modelos sistematicamente com o BSM, e concluir que o modelo alternativo é superior ao descrever as observações reais e possui maior flexibilidade na modelagem dos eventos financeiros. Desta maneira, a maior parte dos modelos com saltos incluem o modelo de Black-Scholes como um caso particular. O universo de modelos de difusão, incluindo os modelos de volatitilidade local e volatilidade estocástica, têm que ser analisados de maneira comparativa aos modelos que incorporam a possibilidade da hipótese de descontinuidade, para que se possa entender as vantagens e desvantagens destes modelos. Esta comparação permite identificar os pontos fortes e fracos dos modelos de difusão e quais propriedades empíricas dos log-retornos dos ativos financeiros são capturados por estes modelos, o que possibilita estabelecer estratégias adequadas de hedge e gerenciamento de risco, que visam mitigar adequadamente, dentro do possível, o risco envolvido com os instrumentos financeiros. Cabe ressaltar que, em certas circunstâncias, os modelos de difusão podem ser calibrados de maneira que capturem boa parte das propriedades genéricas dos modelos com saltos. 


\subsubsection{Modelos e Fatos Estilizados}

Mais interessante do que comparar as trajetórias de movimentos brownianos com as de preços de ativos financeiros, é comparar com o log-retorno do ativo financeiro, i.e., os incrementos nos preços logarítmicos, que são quantidades relevantes para qualquer investidor. Ao estabelecer que a variância do $\mathrm{MB}$ é igual a variância estimada para o processo do log-retorno do ativo financeiro, o que se observa é que o MB atinge esta variância gerando log-retornos que aparentemente parecem ser de mesma amplitude, enquanto que o Ibovespa, em certos momentos, apresenta maiores amplitudes em alguns períodos e menores em outros. Quando a amplitude se eleva de forma considerável, o que se verifica é a presença de saltos nos preços. Esta volatilidade elevada é freqüentemente observada no log-retorno de ativos financeiros. Em termos estatísticos, esta característica resulta em leptocurtose na distribuição empírica dos log-retornos, que no jargão do mercado financeiro é conhecida como caudas grossas, i.e., a cauda da distribuição decai lentamente quando o valor da v.e. tende ao infinito, o que implica em movimentos abruptos nos preços com probabilidade significativa de ocorrência. Este fato, amplamente conhecido, leva a uma desconfiança sobre a hipótese de distribuição gaussiana dos log-retornos, que é uma das propriedades no MB. A distribuição gaussiana atribui às observações, que distam seis desvios-padrão da esperança, probabilidade menor que $10^{-8}$, i.e. praticamente zero. Em um modelo gaussiano de log-retorno diário, uma observação de tal magnitude deveria ocorrer apenas uma vez a cada um milhão de anos, o que não ocorre. Isto implica em um modelo que subestima bastante o risco relacionado aos ativos financeiros.

Os modelos de difusão não-lineares, tais como os apresentados nas equações (0.5) e (0.6), não são processos gaussianos, apesar de serem orientados por ruídos gaussianos. Como é apontado por Nelson (1990) e Sorensen et al. (2005), a escolha apropriada do coeficiente de difusão não-linear combinado com uma tendência determinística (drift) linear pode gerar arbitrariamente um processo de difusão leptocúrtico. Estas observações descartam a possibilidade de rejeitar os modelos de difusão simplesmente pela existência de leptocurtose na distribuição empírica dos log-retonos de ativos financeiros. Entretanto, o único grau de liberdade existente para ajustar o comportamento local de um processo de difusão é o coeficiente de difusão, sendo que a inclusão de leptocur- 
tose é produzida ou pela alta variabilidade nos coeficientes de difusão dos modelos de volatilidade local, ou por valores não condizentes com a realidade da volatilidade da volatilidade dos log-retornos nos modelos de difusão baseados em volatilidade estocástica.

Em contrapartida, o modelo markoviano com saltos mais simples (processos de Lévy), de uma maneira geral, permite alta variabilidade nos log-retornos dos ativos com leptocurtoses similares às das distribuições empíricas, sem a necessidade de incorporar não-estacionariedade, ao escolher valores extremos para os parâmetros do modelo ou adicionar características que não são efetivamente observadas nas séries temporais financeiras. A justificativa para escolha do processo de Lévy, entre todas as possibilidades de processos estocásticos descontínuos para modelar as séries temporais financeiras, é que os processos de Lévy são imprevisíveis (cadlag) e possuem a propriedade de Markov.

Apesar de tudo, o argumento mais forte para adotar modelos descontínuos não é, simplesmente, a presença de saltos nos log-retornos. Enquanto que os modelos de difusão podem gerar leptocurtose nos log-retornos, eles não permitem gerar movimentos descontínuos inesperados no processo dos log-retornos. Em um modelo de difusão, os eventos extremos são resultados da acumulação de vários movimentos pequenos. No caso dos modelos de difusão baseados em volatilidade estocástica, nos quais a volatilidade de mercado varia de forma autônoma, não há mudanças inesperadas, o que implica que os movimentos de curto-prazo no mercado são aproximadamente gaussianas com dimensão do movimento previsível. Um contra-argumento seria que não é possível afirmar que um movimento abrupto é necessariamente uma descontinuidade na trajetória do preço, ou seja, o p.g.d. gera trajetórias contínuas que são observadas de maneira discreta no tempo. Apesar deste argumento ser verdadeiro, deve se levar em consideração que a questão não é se a trajetória dos preços é realmente descontínua ou não, mas sim, propor modelos que reproduzem da maneira mais realista possível as propriedades dos comportamentos dos preços em escalas de tempo de interesse de maneira generalizada, sem a necessidade de estabelecer valores extremos para os parâmetros do processo. Enquanto movimentos abruptos inesperados são propriedades genéricas dos modelos com saltos, eles só podem ser obtidos nos processos de difusão a um custo alto. Em um modelo de difusão o conceito de inesperado, movimento de mercado não-previsível, 
que corresponde à percepção de risco, é difícil de ser obtido, e é neste contexto que os saltos são importantes.

\subsubsection{Hedge e Gerenciamento de Riscos}

Em finanças, modelos de difusão unidimensional (modelos de volatilidade local e estocástica) são exemplos de mercados completos, i.e., qualquer opção pode ser perfeitamente replicada por estratégias auto-financiáveis envolvendo o ativo-objeto e um ativo livre de risco. Em tais mercados, opções são redundantes, pois podem ser substituídas por estratégias de negociação que envolvem o ativo-objeto. Assim, a existência de opções não faz nenhum sentido. Em mercados reais, no entanto, não é possível realizar hedges perfeitos. Desta maneira, as opções permitem aos participantes do mercado hedgear riscos que não poderiam ser hedgeados por estratégias de negociação com o ativo-objeto, que é o propósito que motivou a criação do mercado de derivativos [Ross (1976)].

Enquanto que estes argumentos são reconhecidos por muitos dos operadores de opções que trabalham nas tesourarias de grandes bancos no Brasil, o usual é a aplicação de modelos de difusão, dentro de um contexto de mercados completos, com certas adaptações para as realidades de mercado. O que ocorre, na prática, é um complemento do delta-hedge (aproximação linear) com um gama-hedge (aproximação quadrática). Conforme Arditti (1996), p.76, e Luenberger (1997), p.359, de uma maneira geral, é possível aproximar as variações no preço $f(S, t, r, \sigma)$ de uma opção via expansão de Taylor em torno dos pontos $\left(S_{t}, t, r_{t}, \sigma_{t}\right)$, da seguinte maneira,

$$
d f\left(S_{t}, t, r_{t}, \sigma_{t}\right) \approx \Delta d S_{t}+\frac{1}{2} \Gamma d S_{t}^{2}+\Theta d t+\rho d r_{t}+\Lambda d \sigma_{t}
$$

sendo que $S$ é o preço do ativo objeto, $t$ é o instante de tempo, $r$ é o log-retorno utilizado para descontar o fluxo terminal, $\sigma$ é a volatilidade do ativo objeto $S$, $\left.\Delta \triangleq \frac{\partial f}{\partial S}(S, t, r, \sigma)\right|_{S=S_{t}},\left.\quad \Gamma \triangleq \frac{\partial^{2} f}{\partial S^{2}}(S, t, r, \sigma)\right|_{S=S_{t}},\left.\Theta \triangleq \frac{\partial f}{\partial t}(S, t, r, \sigma)\right|_{t=t}$, $\left.\rho \triangleq \frac{\partial f}{\partial r}(S, t, r, \sigma)\right|_{r=r_{t}} \mathrm{e},\left.\Lambda \triangleq \frac{\partial f}{\partial \sigma}(S, t, r, \sigma)\right|_{\sigma=\sigma_{t}}$. Estas estratégias, que obviamente melhoram o desempenho da replicação dos fluxos de pagamentos terminais, entram em conflito com o próprio modelo, pois a utilização de um modelo de difusão tendo como hipótese o mercado completo, implica reconhecer que as estratégias de hedges gama $(\Gamma)$, vega $(\Lambda)$, pho $(\rho)$ e theta $(\Theta)$ são redundantes às estratégias delta-hedge 
[Cont \& Tankov (2004b), p.12]. Assim, as evidências sugerem que a única forma de hedgear uma opção de compra em relação aos saltos que ocorrem nos preços do ativo-objeto é comprando o próprio ativo-objeto. Desta maneira, não faz nenhum sentido o conceito de replicação do fluxo de pagamentos terminais nos modelos com saltos, pois ele não proporciona a ferramenta adequada para hedgear e gerenciar riscos em mercados incompletos.

Modelos em mercados completos, no qual qualquer ativo contingente pode ser perfeitamente hedgeado por meio de carteiras compostas por posições no ativo-objeto e numerários, não conseguem explicar de maneira plausível a prática usual de hedgear opções exóticas por meio de posições em opções vanilla. Esta prática é facilmente explicada pelos modelos com saltos, nos quais os mercados são incompletos e as opções vanilla não são instrumentos financeiros redundantes. Nestes mercados, as estratégias de hedge não-lineares, tal como o vega-hedge, que permite mitigar parcialmente o risco relacionado aos possíveis saltos que podem ocorrer nos preços dos ativos-objeto. Assim, os modelos de preços descontínuos, de forma geral, não reconhecem a existência de hedges perfeitos, o que implica a existência de hedges com riscos residuais diferentes de zero e que devem ser levados em consideração no gerenciamento de riscos destes investimentos. O Quadro 0.1 resume um pouco do que vem sendo discutido na área de modelagem em finanças de mercado e gerenciamento de risco, e compara os fatos empíricos com as propriedades dos modelos de difusão e os modelos com saltos.

Esta comparação evidencia o problema de pesquisa do estudo: a discrepância entre as hipóteses relacionadas aos modelos utilizados para gerenciamento de risco e precificação de instrumentos financeiros e os fatos empíricos observados nos mercados financeiros.

\subsection{Método de Pesquisa}

O estudo adota um método de pesquisa quantitativa, dispondo definições dos conceitos relevantes e as proposições derivadas destes. Quando for de interesse, serão realizadas as demonstrações matemáticas e estatísticas destes últimos. Adicionalmente, como o estudo é quantitativo e ligado a um problema econômico, i.e, processos dos log- 
Quadro 0.1. Modelando os movimentos de mercado: modelos de difusão versus modelos com saltos

\begin{tabular}{|l|l|l|}
\hline Fatos empíricos & Modelos de difusão & Modelos com saltos \\
\hline $\begin{array}{l}\text { Movimentos abruptos } \\
\text { grandes nos preços }\end{array}$ & $\begin{array}{l}\text { Difícil: necessidade de } \\
\text { volatilidades muito elevadas }\end{array}$ & Propriedade genérica \\
\hline Leptocurtose & $\begin{array}{l}\text { Possível: necessidade de } \\
\text { escolha de estruturas de } \\
\text { volatilidade não-lineares }\end{array}$ & Propriedade genérica \\
\hline $\begin{array}{l}\text { Opções são investimen- } \\
\text { tos arriscados }\end{array}$ & $\begin{array}{l}\text { Opções são hedgeadas de } \\
\text { maneira livre de risco }\end{array}$ & $\begin{array}{l}\text { Hedges perfeitos não } \\
\text { existem }\end{array}$ \\
\hline $\begin{array}{l}\text { Mercados são incom- } \\
\text { pletos, alguns riscos não } \\
\text { podem ser hedgeados }\end{array}$ & Mercados são completos & $\begin{array}{l}\text { Mercados são incom- } \\
\text { pletos }\end{array}$ \\
\hline $\begin{array}{l}\text { Concentração: perdas } \\
\text { são concetradas em } \\
\text { poucos movimentos } \\
\text { grandes negativos }\end{array}$ & $\begin{array}{l}\text { Continuidade: os movimen- } \\
\text { tos dos preços são condicio- } \\
\text { nalmente normais, movi- } \\
\text { mentos abruptos grandes } \\
\text { nào ocorrem }\end{array}$ & $\begin{array}{l}\text { Discontinuidade: saltos } \\
\text { e discontinuidades nos } \\
\text { preços podem gerar } \\
\text { grandes perdas }\end{array}$ \\
\hline $\begin{array}{l}\text { Algumas estratégias de } \\
\text { hedging são melhores } \\
\text { que outras }\end{array}$ & $\begin{array}{l}\text { Todas as estratégias de hedge } \\
\text { resultam em risco residual } \\
\text { zero, independente- } \\
\text { mente da forma que o risco } \\
\text { é mensurado }\end{array}$ & $\begin{array}{l}\text { Estratégias de hedge } \\
\text { são obtidas pela solu- } \\
\text { ção de problemas de } \\
\text { otimização de cartei- } \\
\text { ras }\end{array}$ \\
\hline $\begin{array}{l}\text { Opções exóticas são } \\
\text { hedgeadas através de } \\
\text { opções vanilla (compra } \\
\text { ou venda) }\end{array}$ & $\begin{array}{l}\text { Opços são irredundantes: } \\
\text { qualquer fluxo de pagamen- } \\
\text { tos pode ser replicado por } \\
\text { hedging dinâmicos utilizan- } \\
\text { do o ativo-objeto }\end{array}$ & $\begin{array}{l}\text { Opções não são redun- } \\
\text { dantes: usando opções } \\
\text { vanilla é possível re- } \\
\text { duzir o erro de estra- } \\
\text { tégia de hedge }\end{array}$ \\
\hline
\end{tabular}

retornos de ativos financeiros, o estudo segue uma abordagem econométrica, pois visa unificar de maneira quantitativa aspectos teóricos e empíricos de problemas econômicos [Siqueira (1999), p.12-13]. Visando tornar o texto o mais científico possível, todas as definições, teoremas, proposições, lemas, demonstrações, entre outras, serão indicados claramente no início do parágrafo, permitindo uma fácil referência cruzada, quando estes forem mencionados posteriormente. Pelo mesmo motivo, todas as equações serão devidamente numeradas. Assim, a abordagem adotada no estudo é quantitativa, e sempre que possível serão utilizados métodos estatísticos e matemáticos para apresentar o conteúdo proposto.

\subsection{Revisão Bibliográfica}

As aplicações dos processos de Lévy em finanças já vêm sendo estudadas por alguns autores. Na verdade, o mais antigo processo de Lévy é o movimento browniano, 
que foi inicialmente estudado por Bachelier (1900) e formalizado por Einstein (1905). O trabalho destes dois pesquisadores foi refinado por Osborne (1959), que propôs o exponencial $\exp \left(B_{t}\right)$ do MB para modelar o preço de uma ação. Ele justificou a sua abordagem com argumentos psicológicos, astronômicos e fisiológicos baseados na lei Weber-Fechner, que sugere que os seres humanos percebem a intensidade dos estímulos por meio de escalas logarítmicas, ao contrário de escalas lineares. De uma maneira mais sistemática, este mesmo processo $\exp \left(B_{t}\right)$, denominado $\mathrm{MBG}$, foi apresentado por Samuelson (1965).

Um dos primeiros autores a propor um processo de Lévy exponencial não gaussiana foi Mandelbrot (1963). Ele observou que o logaritmo das variações relativas do preços nos mercados financeiros e de commodites exibiam leptocurtose em suas distribuições. A sua conclusão foi de que o MBG deveria ser substituído por movimentos de Lévy $\alpha$-estáveis, com índice $\alpha<2$. Esta distribuição resultava em processos construídos por saltos para os preços de ações. A distribuição gaussiana é um caso particular da distribuição $\alpha$-estável, quando $\alpha=2$. Assim, o modelo de Mandelbrot era complementar ao modelo desenvolvido por Osborne (1959) ou Samuelson (1965). Alguns anos depois, um processo exponencial de Lévy com distribuição não-estável foi proposto por Press (1967). O modelo proposto por Press considerava o processo do logretorno como a sobreposição do $\mathrm{MB}$ ao processo independente de Poisson com saltos distribuídos gaussianamente. A motivação deste era achar um modelo que se ajustasse melhor às distribuições empíricas observadas nas variações do log-retorno de ações (taxa de retorno diária e intra-diária composta continuamente). Praetz (1972) também contribui para o estudo do processo do log-retorno ao mostrar por meio de dados empíricos que a distribuição do log-retorno é leptocúrtica.

No final da década de 80 , Madan \& Seneta (1987) propuseram um processo de Lévy para o log dos preços de ativos financeiros, cujos incrementos possuíam distribuição variância gama ${ }^{1}$. Esta escolha era justificada pelo estudo estatístico de dados de mercado da Bolsa de Valores da Austrália. De forma análoga ao movimento de

1 A função característica [Definição C.1] que define a distribuição variância gama $V G(\sigma, \theta, \nu, \mu)$ é

$$
\Phi_{V G(\sigma, \theta, \nu, \mu)}(u)=\exp (i \mu u)\left(\frac{1}{1-i \theta \nu u+\left(\sigma^{2} \nu / 2\right) u^{2}}\right)^{1 / \nu} .
$$


Lévy $\alpha$-estavél, os processos de Lévy variância gama são essencialmente compostos puramente por saltos. Entretanto, este último possui função geratriz de momentos (m.g.f.) [Definição C.2], o que é conveniente para fins de modelagem em finanças. Em particular, ao escolher parâmetros adequados para a distribuição variância gama, a esperança dos preços dos ativos existem [Madan \& Seneta (1987)]. Distribuições variância gama são casos particulares (limites) das distribuições da família de distribuições hiperbólica generalizada. Esta família de distribuições foi inicialmente introduzida por Barndorff-Nielsen (1977) para modelar a distribuição de tamanhos de grãos de areia soprados pelo vento. Duas classes de distribuições hiperbólicas generalizadas se mostraram bastante eficientes para modelar as distribuições empíricas dos log-retornos diários e intra-diários de ativos financeiros. A primeira classe foi apresentada por Eberlein \& Keller (1995), que introduziu o movimento hiperbólico exponencial de Lévy para modelar o preço de ativos financeiros e, a segunda classe foi apresentada por Barndorff-Nielsen (1997), que propôs um processo de Lévy exponencial invertido gaussiano. Nos anos seguintes, Prause (1999) e Eberlein \& Prause (2000) efetuaram um estudo completo da família hiperbólica generalizada de processos de Lévy. No Brasil pouco foi feito, apenas Fajardo et al. (2001) buscou entender a magnitude e importância do trabalho de Prause (1999).

Por outro lado, a pesquisa mais aprofundada de fatos empíricos das séries temporais financeiras permeia sobre boa parte da literatura moderna de finanças e econometria financeira, sendo que as principais sínteses foram realizadas por Pagan (1996), Campbell et al. (1997), Cont (2001) e Gourieroux (2001). 


\section{Capítulo 1 Processos e Medidas Estocásticas}

Um processo estocástico é uma família de variáveis estocásticas $\left\{X_{t}\right\}_{t \in[0 ; T]}$ indexadas no tempo. O parâmetro $t$ pode ser discreto ou contínuo. Para cada realização de um cenário estocástico $\omega$, a trajetória $X(\omega): t \longrightarrow X_{t}(\omega)$ define uma função do tempo.

Para interpretar o indexador $t$ (tempo) é necessário levar em consideração, que quanto maior for a quantidade de informação disponível ao longo do tempo, menor a incerteza em relação a um determinado evento futuro. Assim, parece intuitivo a importância com que a informação é revelada no tempo. Esta intuição está relacionada com a idéia de filtragem.

\subsection{Processos e Funções Estocásticas}

Um processo estocástico pode ser entendido como uma função $X:[0 ; T] \times \Omega \longmapsto$ $E$ que dependente tanto do tempo $t$, como do cenário estocático $\omega$. Assim, o processo estocástico pode ser entendido como uma função estocástica, i.e., variáveis estocásticas assumindo valores nos espaços em que a função é definida. A escolha mais simples de função espacial para um processo estocástico é aquela definida em $\mathbb{R}^{d}$, ou seja, $f:[0 ; T] \longrightarrow \mathbb{R}^{d}$. O conjunto $\mathbb{R}^{d}$ parece ser um pouco grande e contém funções problemáticas que podem ser difíceis de serem mensuradas. Processos estocásticos podem ser construídos através de funções estocásticas definidas no espaço de funções contínuas $\mathcal{C}\left([0 ; T], \mathbb{R}^{d}\right)$. A topologia usual deste espaço é definida como

$$
\|f\|_{\infty} \triangleq \sup _{t \in[0 ; T]}\|f(t)\|
$$

que por sua vez pode ser utilizado para construir uma $\sigma$-álgebra de Borel, nos quais as medidas podem ser definidas. Um exemplo interessante é a medida de Wiener, i.e., a medida gaussiana definida em $\mathcal{C}\left([0 ; T], \mathbb{R}^{d}\right)$ que descreve o processo de Wiener. Apesar disto, muitos processos de interesse não serão contínuos, pois apresentarão saltos 
(jumps), durante o caminho observado do processo. A classe de funções cadlag $^{2}$ parece ser a classe de funções descontínuas mais apropriada para a modelagem em finanças.

Definição 1.1 Função Cadlag A função $f:[0 ; T] \longrightarrow \mathbb{R}^{d}$ é uma função cadlag se ela for contínua à direita com limites à esquerda. Para qualquer $t \in[0 ; T]$ os limites

$$
f(t-)=\lim _{s \uparrow t} f(s) \quad f(t+)=\lim _{s \downarrow t} f(s)
$$

existem, e $f(t)=f(t+)$.

Qualquer função contínua é uma função cadlag, mas nem todas as funções cadlag são contínuas. Se $t$ é um um ponto de descontinuidade, então,

$$
\Delta f(t)=f(t)-f(t-)
$$

é um salto de $f$ em $t$. Uma função cadlag não pode ter descontinuidades desenfreadamente. $\mathrm{O}$ número de descontinuidades grandes deve ser finito, i.e., $\{t \in[0 ; T], f(t) \neq$ $f(t-)\}$ é finito. Assim, para qualquer $\epsilon>0$, o número de descontinuidades (saltos) em $[0 ; T]$ maior que $\epsilon$ deve ser finito. Desta maneira, uma função cadlag em $[0 ; T]$ tem uma quantidade finita de saltos grandes (maiores que $\epsilon$ ), e possivelmente uma quantidade infinita de saltos pequenos (menores ou iguais a $\epsilon$ ). Esta propriedade é decorrente da singulariedade que ocorre quando o tamanho dos saltos tendem a zero, como será observado mais adiante.

Um exemplo de função cadlag é a função salto que apresenta um salto em um ponto qualquer $T_{0}$. O valor da função em $T_{0}$ é definido pelo valor da função após o salto, i.e., $f=1_{\left[T_{0} ; T[\right.}(t)$. Neste caso, $f\left(T_{0}-\right)=0, f\left(T_{0}+\right)=f\left(T_{0}\right)=1$ e $\Delta f\left(T_{0}\right)=1$. De uma forma geral, dada uma função contínua $g:[0 ; T] \longrightarrow \mathbb{R}$ e constantes $f_{i}$, para $i=0,1, \ldots, n-1$ e $0<t_{1}<t_{2}, \ldots,<t_{n}=T$, a função a seguir é uma função cadlag:

$$
f(t)=g(t)+\sum_{i=0}^{n-1} f_{i} 1_{\left[t_{i} ; t_{i+1}[\right.}(t) .
$$

A função $g$ pode ser interpretada como a componente contínua de $f$ aos quais os saltos são adicionados, i.e., os saltos de $f$ ocorrem em $t_{i}, i \geq 1 \operatorname{com} \Delta f\left(t_{i}\right)=f_{i}-f_{i-1}$. Cabe ressaltar que, nem toda função cadlag possui a clara decomposição em componentes

2 A palavra "cadlag" tem oridem do acrônimo francês continu à droite, limite à gauch. Outra notação equivalente é "rcll" que é o acrônimo, proveniente do inglês, right-continuous with left limits. 
contínuos e saltos. As funções cadlag são trajetórias naturais de processos estocásticos com saltos (jump). Neste exemplo, a função é contínua à direita e apresenta saltos em $t_{i}$, simplesmente porque foi convencionado que o valor da função será o valor após o salto, i.e., $f\left(t_{i}\right):=f\left(t_{i}+\right)$. Se, por acaso, for estabelecido que o valor da função será o valor antes do salto, i.e., $f\left(t_{i}\right):=f\left(t_{i}-\right)$, então, a função será contínua à esquerda com limites à direita (caglad). Esta diferença, é significante no sentido que $t$ é entendida como a variável tempo, e o termo "à direita " significa depois, enquanto "à esquerda" significa antes. Se a função for contínua à direita com saltos em $t$, então, o valor da função $f(t)$ não é previsível até o instante de tempo $t$. Por outro lado, se a função for contínua à esquerda, ao se aproximar de $t$ durante a trajetória da função, é possível prever qual o valor em $t$. No contexto de modelagem em finanças, saltos representam eventos que ocorrem inesperadamente e que não podem ser previstos, o que implica na escolha de funções contínuas à direita. Entretanto, se o intuito for a modelagem de processos descontínuos, cujos os valores são previsíveis, então, a escolha certa seria funções caglad.

É possível definir uma topologia e conceito de convergência para os espaço das funções cadlag. Para maior detalhes vide Jacod \& Shiryaev (2002) e Shiryaev \& Prokhorov (1989) Ao equipar os processos com esta topologia e a correspondente $\sigma$-álgebra de Borel, o espaço das funções cadlag é denominado por Espaço de Skorokhod e será representado por $\boldsymbol{D}\left([0 ; T], \mathbb{R}^{d}\right)$, ou simplesmente $\boldsymbol{D}([0 ; T])$, onde $\mathcal{C}([0 ; T]) \subset \boldsymbol{D}([0 ; T])$. Uma v.e. com valores definido em $\boldsymbol{D}([0 ; T])$ será um processo cadlag. Assim, ao trabalhar com processos cadlag, fica subentendido que o espaço das trajetórias do processo é $\boldsymbol{D}([0 ; T])$.

\subsubsection{Filtragem e História}

O entendimento do índice $t$, como a variável tempo, introduz a dinâmica que deve ser levada em consideração para definir os conceitos de informação, casualidade e previsibilidade, no contexto dos processos estocásticos.

À medida que o tempo passa, uma maior quantidade de informação é revelada ao observador do processo. Assim, o status desta informação, que em $t=0$, quando a informação é revelada, é considerado um evento estocástico, muda em $t>0$. Desta 
maneira, o espaço probabilístico $(\Omega, \mathcal{F}, \mathbb{P})$ deve incorporar a dependência temporal do processo. Esta característica é denominada filtragem.

Definição 1.2 Filtragem Uma filtragem ou fluxo de informação em $(\Omega, \mathcal{F}, \mathbb{P})$ é uma família crescente de $\sigma$-álgebras $\left\{\mathcal{F}_{t}\right\}_{t \in[0 ; T]}: \forall t \geq s \geq 0, \mathcal{F}_{s} \subseteq \mathcal{F}_{t} \subseteq \mathcal{F}$

$\mathcal{F}_{t}$ deve ser interpretado como a informação conhecida até o momento $t$, sendo crescente ao longo do tempo. Um espaço probabilístico $(\Omega, \mathcal{F}, \mathbb{P})$ equipado de uma filtragem é chamado de espaço probabilístico filtrado. Parece intuitivo que a probabilidade de ocorrência de um evento estocástico muda à medida que uma maior quantidade de informação está disponível. Entretanto, em vez de mudar com o tempo a medida probabilística $\mathbb{P}, \mathbb{P}$ fica inalterada, e o impacto da informação revelada ao longo do tempo é capturada pelo condicionamento da filtragem ao tempo: $\mathcal{F}_{t}$.

O fluxo de informação descrito pela filtragem $\mathcal{F}_{t}$ distingue a quantidade de informação que já foi revelada até o instante $t$, da quantidade de informação que ainda será revelada após o instante $t$, ou seja, da informação que é considerada como eventos estocásticos no instante $t$. Um evento $A \in \mathcal{F}_{t}$ é um evento, que dada a informação em $\mathcal{F}_{t}$ no instante $t$, já ocorreu. De maneira análoga, uma v.e. $\mathcal{F}_{t}$-mensurável é uma v.e. cujo valor será revelado no instante $t$. Um processo cujo valor no instante $t$ é revelado pela informação contida em $\mathcal{F}_{t}$, é um processo não-antecipável.

Definição 1.3 Processo Não-Antecipável Um processo estocástico $\left\{X_{t}\right\}_{t \in[0 ; T]}$ é não-antecipável em relação a uma estrutura de informação $\left\{\mathcal{F}_{t}\right\}_{t \in[0 ; T]}$ ou $\mathcal{F}_{t}$-adaptável se, para qualquer $t \in[0 ; T]$, o valor de $X_{t}$ é revelado no instante $t: a$ v.e. $X_{t}$ é $\mathcal{F}_{t}$-mensurável.

Um processo não-atencipável é um processo adaptável, i.e., $\left\{X_{t}\right\}_{t \in[0 ; T]}$ é $\mathcal{F}_{t}$-adaptável. O termo adaptável é menos intuitivo que o termo não-antecipável, apesar de ser mais usual nos livros de cálculo estocástico. Se a única observação disponível são os valores passados do processo estocástico $X$, então, a informação representa a história de $X$. 
Definição 1.4 História do Processo A história do processo $X$ é filtragem $\left\{\mathcal{F}_{t}^{X}\right\}_{t \in[0 ; T]}$, onde $\mathcal{F}_{t}^{X}$ é a $\sigma$-álgebra gerada pelos valores passados do processo, $e$ completado pelos conjuntos nulos $(\mathbf{n})$ :

$$
\mathcal{F}_{t}^{X}=\sigma\left(X_{s}, s \in[0 ; t]\right) \vee \mathbf{n} .
$$

$\mathcal{F}_{t}^{X}$ deve ser entendido como o objeto que contém toda a informação que se pode extrair do caminho observado de $X$, no intervalo de tempo $[0 ; t]$. No caso da modelagem em finanças, é possível construir a história do conjunto de preços de ativos de interesse, baseado na filtragem do preço destes ativos. Cabe ressaltar que, $\mathcal{F}_{t}^{X}$ é completa pois foram adicionados todos os conjuntos nulos $\mathbf{n}$, i.e., todos os conjuntos nulos estão embutidos em $\mathcal{F}_{0}$. Isto significa que, se um percurso de $X$ em $t \in[0 ; T]$ é impossível, então, este percurso já conhecido a priori em $t=0$.

\subsubsection{Tempo Estocástico}

Ao modelar eventos financeiros, haverá a necessidade de modelar eventos onde o tempo é estocástico. Um tempo estocástico é uma v.e., que assume valores positivos, $T \geq 0$, e representa o instante em que o evento ocorreu (ou ocorrerá). Dado uma filtragem $\left\{\mathcal{F}_{t}\right\}$, é possível verificar se um evento está contido em $\mathcal{F}_{t}$, i.e., se o evento ocorreu $(\tau \leq t)$ ou ainda irá ocorrer $(\tau>t)$. Se o evento já tiver ocorrido, o tempo estocástico $\tau$ é uma v.e. não-antecipável, que também é denominada tempo de parada. Em outras palavras, $T$ é um tempo estocástico não-atencipável $\left(\left\{\mathcal{F}_{t}\right\}\right.$-tempo de parada) se

$$
\forall t \geq 0, \quad\{T \leq t\} \in \mathcal{F}_{t}
$$

Se $T_{1}$ e $T_{2}$ são tempos de parada, então, $T_{1} \wedge T_{2}=\inf \left\{T_{1}, T_{2}\right\}$ também será um tempo de parada. O termo tempo de parada parece indicar que algo irá parar no instante $\tau$, i.e., dado um tempo de parada $\tau$ e um processo não-antecipável $\left\{X_{t}\right\}$ é possível estabelecer um outro processo $X_{\tau \wedge t}$, que é o processo $X$ com parada no instante $\tau$ :

$$
\left\{\begin{array}{l}
X_{\tau \wedge t}=X_{t}, \text { se } t<\tau \\
X_{\tau \wedge t}=X_{\tau}, \text { se } t \geq \tau .
\end{array}\right.
$$

Um exemplo de tempo de parada é o processo cadlag não-antecipável $T_{A}$, onde o tempo de parada deste processo ocorre quando $X$ atinge pela primeira vez algum valor 
do conjunto aberto $A$, i.e.,

$$
T_{A}=\inf \left\{t \geq 0, X_{t} \in A\right\} \text {. }
$$

A qualquer instante $t$, é possível verificar qual os valores realizados por $X_{t}$ e saber se o conjunto $A$ foi alcançado $\left(T_{A} \leq t\right)$ ou não $\left(T_{A}>t\right)$, este exemplo é conhecido como tempo de alcance (hitting times). Outro exemplo, conhecido como tempo de saída (exit time): $X$ é um processo, de valor definido nos reais, começando em $t=0$, e $T_{a}>0$ é o tempo de saída de $X$ do intervalo ] $-\infty ; a]$, i.e.,

$$
T_{a}=\inf \left\{t>0, X_{t}>a\right\} \text {. }
$$

Este tempo de parada é igual ao exemplo do tempo de alcance para um $T_{A}$ associado à um intervalo aberto $A=] a ; \infty[$. Um exemplo de tempo estocástico interessante, que não é considerado como um tempo de parada é o primeiro instante de tempo $t \in[0 ; T]$ que $X$ atinge o máximo:

$$
T_{\max }=\inf \left\{t \in[0 ; T], X_{t}=\sup _{s \in[0 ; T]} X_{s}\right\} .
$$

Para que o máximo ocorra, é necessário saber a priori qual o valor máximo que o processo pode assumir no intervalo de tempo $t \in[0 ; T]$. Assim, dada a informação contida em $\mathcal{F}_{t}$ no instante $t<T$ não é possível saber se $T_{\max }$ ocorreu ou não, isto só é possível em $t=T$.

Dado uma filtragem $\mathcal{F}_{t}$, e um tempo estocástico não-antecipável $\tau$, o conjunto informacional $\mathcal{F}_{\tau}$ pode ser entendido como a informação obtida do processo cadlag nãoantecipável $X$ observado no instante $\tau$, i.e., a $\sigma$-álgebra gerada pelo processo observado $\mathcal{F}_{\tau}=\sigma\left(X_{\tau}, X\right.$ cadlag não-antecipável $)$. Esta definição é equivalente à apresentada por Protter (1990), p.3:

$$
\mathcal{F}_{\tau}=\left\{A \in \mathcal{F}, \forall t \in[0 ; T], A \cap\{t \leq \tau\} \in \mathcal{F}_{t}\right\} .
$$

\subsubsection{Martingales}

Ao considerar um espaço probabilístico $(\Omega, \mathcal{F}, \mathbb{P})$ equipado com uma filtragem $\mathcal{F}_{t}$. 
Definição 1.5 Martingale Um processo cadlag $\left\{X_{t}\right\}_{t \in[0 ; T]}$ é uma martingale se $X$ é $\mathcal{F}_{t}$-adaptável (não-antecipável), $\mathbb{E}\left[\left|X_{t}\right|\right]<\infty$ para qualquer $t \in[0 ; T]$, e

$$
\forall s>t, \quad \mathbb{E}\left[X_{s} \mid \mathcal{F}_{t}\right]=X_{t} . \quad \text { q.c. }
$$

Em outras palavras, a melhor previsão do valor futuro de uma martingale é o seu valor presente. Um exemplo conhecido de martingale é o processo de Wiener $\left\{W_{t}\right\}$. Cabe ressaltar que, a definição de martingale faz sentido apenas no contexto da filtragem $\left\{\mathcal{F}_{t}\right\}_{t \in[0 ; T]}$ e da existência de uma medida probabilística $\mathbb{P}$. Para evitar qualquer confusão, podemos escrever estes elementos como $\left(\mathcal{F}_{t}, \mathbb{P}\right)$-martingale. Quando várias medidas probabilísticas estão sendo analisadas, podemos utilizar o termo $\mathbb{P}$-martingale para enfatizar que o conceito de martingale depende da medida probabilística $\mathbb{P}$. Considerere a existência de um v.e. $H$, revelada no instante $T$, i.e., $\mathcal{F}_{T}$-mensurável, com $\mathbb{E}[|H|]<\infty$, o processo $\left\{M_{t}\right\}_{t \in[0 ; T]}$, será uma martingale se $M_{t}=\mathbb{E}\left[H \mid \mathcal{F}_{t}\right]$ é uma martingale. De forma análoga, qualquer martingale $\left\{M_{t}\right\}_{t \in[0 ; T]}$ pode ser escrito desta forma, se $H=M_{T}$ (valor terminal) ${ }^{3}$. Uma conseqüência do resultado (1.11) é que uma martingale possui esperança constante, i.e., $\forall t \in[0 ; T], \mathbb{E}\left[X_{t}\right]=\mathbb{E}\left[X_{0}\right]$. Nem todos os processos que apresentam tendências determinísticas [p. 167, Enders (2003)] iguais a zero (driftless) são martingales. Um exemplo é o processo de Wiener $W=\left\{W_{t}\right\}$. Neste processo, $\left\{W_{t}^{3}\right\}$ possui esperança constante $\mathbb{E}\left[W_{t}^{3}\right]=0$, mas não é uma martingale. Os dois primeiros momentos condicionados do processo de Wiener são, respectivamente, $\mathbb{E}\left[W_{s}-W_{t} \mid \mathcal{F}_{t}\right]=0$ e $\mathbb{E}\left[\left(W_{s}-W_{t}\right)^{2} \mid \mathcal{F}_{t}\right]=s-t$, para $s>t$. Assim, se $s>t$, então,

$$
\begin{aligned}
\mathbb{E}\left[W_{s}^{3} \mid \mathcal{F}_{t}\right]= & \mathbb{E}\left[\left(W_{s}-W_{t}+W_{t}\right)^{3} \mid \mathcal{F}_{t}\right] \\
= & \mathbb{E}\left[\left(W_{s}-W_{t}\right)^{3}+W_{t}^{3}+3\left(W_{s}-W_{t}\right) W_{t}^{2}+3\left(W_{s}-W_{t}\right)^{2} W_{t} \mid \mathcal{F}_{t}\right] \\
= & \mathbb{E}\left[\left(W_{s}-W_{t}\right)^{3} \mid \mathcal{F}_{t}\right]+W_{t}^{3}+3 W_{t}^{2}\left(\mathbb{E}\left[W_{s} \mid \mathcal{F}_{t}\right]-W_{t}\right) \\
& +3 W_{t} \mathbb{E}\left[\left(W_{s}-W_{t}\right)^{2} \mid \mathcal{F}_{t}\right] \\
= & 0+W_{t}^{3}+0+3 W_{t}(s-t) \neq W_{t}^{3} . \quad \text { q.c. }
\end{aligned}
$$

3 Cabe ressaltar que, os processos e martingales são definidos em intervalos de tempo finito $t \in[0 ; T]$. Os resultados apresentados neste estudo não se extendem para $t \in[0 ; \infty[$. 
Entretanto, ao analisar um processo sem t.d. quando o tempo é estocástico, esta característica é suficiente para caracterizar uma martingale [Protter (2001)], i.e., se $\mathbb{E}\left[X_{\tau}\right]=$ $\mathbb{E}\left[X_{0}\right]$ para qualquer tempo de parada $\tau$, então, $X$ é uma martingale.

Uma propriedade fundamental das martingales é a propriedade de amostragem. Esta propriedade refere-se à validade do resultado (1.11), ao substituir $t$ e $s$ por tempos estocásticos não-antecipáveis.

Proposição 1.1 Teorema da Amostragem $S e\left\{M_{t}\right\}_{t \in[0 ; T]}$ é uma martingale, e, $T_{1}$ e $T_{2}$ são tempos estocásticos (tempos de parada) não-antecipáveis, com $T \geq T_{2} \geq$ $T_{1} \geq 0$ q.c., então,

$$
\mathbb{E}\left[M_{T_{2}} \mid \mathcal{F}_{T_{1}}\right]=M_{T_{1}} . \quad \text { q.c. }
$$

Esta proposição é também apresentada por alguns pesquisadores na forma de teorema [Protter (1990), p.10]. Desta maneira, uma martingale com tempo de parada (tempo estocástico) não-antecipável também é uma martingale.

O processo $\left\{X_{t}\right\}_{t \in[0 ; T]}$ é denominado martingale local, se existir uma seqüência de tempos de parada $\left\{T_{n}\right\}_{n \in \mathbb{N}}$ para $T_{n} \longrightarrow \infty$ q.c., tal que $\left\{X_{t \wedge T_{n}}\right\}_{t \in[0 ; T]}$ é uma martingale. Assim sendo, uma martingale local se comporta como uma martingale até o tempo de parada $T_{n}$, que pode ser tão grande quanto for desejável. Como conseqüência, tem-se que qualquer martingale é uma martingale local, entretanto, existem martingales locais que não são martingales. Esta sutileza é de grande importância para a Teoria Martingale aplicada à modelagem em finanças.

\subsubsection{Processo Previsível}

Os processos $\left\{X_{t}\right\}_{t \in[0 ; T]}$ já discutidos neste estudo são, ou função do tempo para um determinado $\omega$, i.e., o caminho estocástico observado $t \longmapsto X_{t}(\omega)$, ou função de $\omega$ para um instante de tempo determinado $t$, i.e., a v.e. $\omega \longmapsto X_{t}(\omega)$. Parece interessante analisar estes dois aspectos ( $\omega$ e $t$ ) em conjunto. Para isto, é preciso considerar $X$ como sendo uma função definida em $[0 ; T] \times \Omega$. Esta representação exige que a $\sigma$-álgebra gerada por este processo seja definida como uma função mensurável em $[0 ; T] \times \Omega$, conforme Definição B.3. Inicialmente, é possível considerar que a $\sigma$-álgebra, gerada pelo 
produto $B \times A$, no qual $A \subset \mathcal{F}$ e $B \subset[0 ; T]$ é mensurável. Dada a filtragem $\left\{\mathcal{F}_{t}\right\}_{t \in[0 ; T]}$ é de grande interesse que a classe de processos cadlag adaptáveis (não-antecipáveis) sejam funções mensuráveis em $[0 ; T]$.

Definição 1.6 Processos Opcionais ${ }^{4} \quad A$-álgebra $\mathcal{O}$ em $[0 ; T] \times \Omega$ gerada por processos cadlag adaptáveis é uma $\sigma$-álgebra opcional. Um processo $X:[0 ; T] \times$ $\Omega \longmapsto \mathbb{R}^{d}$, que é mensurável em relação a $\sigma$-álgebra $\mathcal{O}$, é um processo opcional.

Dada a Definição 1.6, qualquer processo cadlag adaptável é um processo opcional. Entretanto, podem existir outros processos que não são cadlag que geram processos opcionais. Assim, processos cadlag adaptáveis geram processos opcionais da mesma maneira que, funções contínuas geram funções mensuráveis. Dado a distinção entre funções contínuas à direita e contínuas à esquerda, é necessário definir um outro tipo de $\sigma$-álgebra:

\section{Definição 1.7 Processos Previsíveis $\quad A$-álgebra $\mathcal{P}$ em $[0 ; T] \times \Omega$ gerada por} processos caglad adaptáveis é uma $\sigma$-álgebra previsível. Um processo $X:[0 ; T] \times$ $\Omega \longmapsto \mathbb{R}^{d}$, que é mensurável em relação a $\sigma$-álgebra $\mathcal{P}$, é um processo previsível.

O termo previsível é justificável pela própria definição de funções contínuas à esquerda. Qualquer processo contínuo à esquerda é, portanto, previsível, i.e., intuitivamente, se $\lim _{s \uparrow t} X_{s}=X_{t}$, então, o valor $X_{t}$ é divulgado pelos valores instantâneos precedentes. No sentido da Definição 1.7, todos os processos gerados por processos contínuos à esquerda são previsíveis (da mesma forma que os conjuntos Borel são gerados pelos intervalos abertos). Apesar disto, podem existir outros processos que não são contínuos à esquerda que geram processos previsíveis. É possível sintetizar estas duas últimas definições da seguinte maneira:

1. Cadlag e Contínuo à direita + adaptável $\Longrightarrow$ Opcional

2. Caglad e Contínuo à esquerda + adaptável $\Longrightarrow$ Previsível

4 Cabe ressaltar que o termo opcional não tem nenhuma relação com os instrumentos financeiros denominados opções. 
A diferença entre processos opcionais e previsíveis é importante no contexto da modelagem em finanças, pois as variáveis que indicam estados da natureza, tais como os preços de mercado serão modelados como processos opcionais, e as decisões financeiras, tais como estratégias de hedge e de investimentos em carteiras, serão modelados por processos previsíveis.

\subsection{Processo de Difusão}

\subsubsection{Propriedades Markovianas}

Processo de difusão, ou simplesmente difusão, está relacionado com o estudo da existência e unicidade de soluções para equações diferenciais estocásticas (SDE). De uma maneira geral, o termo difusão é atribuído à processos de Markov com trajetórias contínuas que podem ser caracterizados pelo seu gerador infinitesimal. O estudo de difusões introduz a necessidade das seguintes definições:

Definição 1.8 Processo de Markov Seja d um inteiro positivo e $\mathbb{P}$ uma medida probabilística definida no espaço mensurável $\left(\mathbb{R}^{d}, \mathcal{B}\left(\mathbb{R}^{d}\right)\right)$ [Subseção C.1]. O processo $X=\left\{X_{t}, \mathcal{F}_{t}\right\}_{t \geq 0}, \mathcal{F}_{t}$-adaptável, definido no espaço probabilístico $(\Omega, \mathcal{F}, \mathbb{P})$ é um processo de Markov se e somente se:

1. Para qualquer $A \in \mathcal{F}$, a função

$$
\begin{aligned}
\mathbb{P}: \mathcal{F} & \longrightarrow[0 ; 1] \\
A & \longmapsto \mathbb{P}(A)
\end{aligned}
$$

é mensurável;

2. $\mathbb{P}\left(X_{0}=x\right)=1, \forall x \in \mathbb{R}^{d}$.

3. $\operatorname{paras}, t \geq 0$ e $A \in \mathcal{B}\left(\mathbb{R}^{d}\right)$,

$$
\mathbb{P}\left[X_{t+s} \in A \mid \mathcal{F}_{s}\right]=\mathbb{P}\left[X_{t+s} \in A \mid X_{s}\right], \mathbb{P} \text {-q.c. }
$$


A propriedade 3 da Definição 1.8, dada a sua importância na definição, é denominada propriedade de Markov. Intuitivamente, um processo de Markov é aquele cuja previsão de sua trajetória futura condicionada a um conjunto informacional, que inclui toda informação passada e presente $\mathcal{F}_{s}$, dependa apenas da informação presente $X_{s}$. Outra definição de interesse para o estudo de difusões é a de um processo forte de Markov:

Definição 1.9 Processo Forte de Markov O processo $X=\left\{X_{t, \tau}, \mathcal{F}_{t}\right\}_{t \geq 0}$ de dimensão d, $\mathcal{F}_{t}$-adaptável, definido no espaço probabilístico $(\Omega, \mathcal{F}, \mathbb{P})$ é um processo forte de Markov se e somente se:

1. For um processo de Markov.

2. $\quad$ para qualquer tempo de parada finito $\tau<\infty$ adaptado em $\left\{\mathcal{F}_{t}\right\}_{t \geq 0}, t \geq 0 e$ $A \in \mathcal{B}\left(\mathbb{R}^{d}\right)$,

$$
\mathbb{P}\left[X_{\tau+t} \in A \mid \mathcal{F}_{\tau}\right]=\mathbb{P}\left[X_{\tau+t} \in A \mid X_{\tau}\right], \mathbb{P}-q . c .
$$

O conceito subjacente ao processo forte de Markov é semelhante à propriedade de Markov, só que garantida quando o tempo é estocástico, i.e., $t$ é substituído por $\tau$. A propriedade forte de Markov [propriedade 2 da Definição 1.9] implica na propriedade tempo homogêneo, que pode ser escrita como:

$$
\mathbb{P}\left[X_{s+t}\right]=\mathbb{P}\left[X_{s}\right]+\mathbb{P}\left[X_{t}\right]
$$

\subsubsection{Movimento Browniano}

O movimento browniano (MB) foi proposto, em 1828, pelo botânico escocês Robert Brown durante suas pesquisas sobre os movimentos irregulares de grãos de pólem suspensos em líquidos e, posteriormente, formulado matematicamente por Eisntein em 1905. O MB pode ser definido como:

Definição 1.10

Movimento Browniano

Seja um espaço probabilístico

$(\Omega, \mathcal{F}, \mathbb{P})$ e $B=\left\{B_{t}, \mathcal{F}_{t}\right\}_{t \geq 0}$ em $\mathbb{R}^{d}$ um processo $\mathcal{F}_{t}$-adaptável definido neste espaço. $B$ é um movimento browniano $(M B)$ de dimensão d se: 
1. Para qualquer $0 \leq s<t<\infty$, os incrementos do processo são independentes de sua filtragem, i.e., $B_{t}-B_{s}$ é independente de $\mathcal{F}_{s}$.

2. Para qualquer $0 \leq s<t$, os incrementos são variáveis estocásticas gaussianamente distribuídas, com média zero e matriz de covariância $(t-s) \Sigma$, para uma determinada matriz estocástica $\Sigma$, i.e., $B_{t-s} \stackrel{d}{=} \mathcal{N}[0 ;(t-s) \Sigma]$.

3. O valor incial do processo é igual a $x$-q.c., i.e., $\mathbb{P}\left[B_{0}=x\right]=1$.

$S e \mathbb{E}\left[\left|B_{0}\right|\right]<\infty$, então, o MB é um processo martingale. Os movimentos brownianos de trajetórias contínuas, i.e., os processos $B$ definidos em $\mathcal{C}\left(\left[0 ; \infty\left[, \mathbb{R}^{d}\right)\right.\right.$ são denominados movimentos brownianos canônicos [Øksendal (2003), p.12]. Neste estudo, deve ficar subentendido que ao se referir ao MB está se referindo ao movimento browniano canônico. Se a matriz $\Sigma$ for uma matriz identidade de dimensão d, i.e., $\Sigma=I$, então, o MB é denominado processo de Wiener $W=\left\{W_{t}\right\}_{t \geq 0}$ ou movimento browniano padronizado, e sua distribuição, para qualquer $0 \leq s<t$, é $W_{t-s} \stackrel{d}{=} \mathcal{N}[0 ;(t-s) I]$.

\subsubsection{Processo de Difusão de Itô}

Conforme Karatzas \& Shreve (1997), p.281, a definição de um processo de difusão é:

\section{Definição 1.11 Processo de Difusão $O$ processo forte de Markov} $X=\left\{X_{t}, \mathcal{F}_{t}\right\}_{t \geq 0}$ de dimensão $d, \mathcal{F}_{t}$-adaptável, definido no espaço probabilístico $(\Omega, \mathcal{F}, \mathbb{P})$ é uma difusão se e somente se:

1. As trajetórias do processo $X$ forem contínuas.

2. O limite à direita em cada ponto $x$ da trajetória é

$$
\lim _{t \downarrow 0} \frac{1}{t}\left[\mathbb{E}\left[f\left(X_{t+s}\right) \mid X_{s}=x\right]-f(x)\right]=(\mathcal{A} f)(x), x \in \mathbb{R}^{d}
$$

para qualquer $f \in \mathcal{C}^{2}\left(\mathbb{R}^{d}\right)$. 
3. O operador $(\mathcal{A} f)(x)$ é denominado gerador infinitesimal do processo e é igual a

$$
(\mathcal{A} f)(x)=\frac{1}{2} \sum_{i, k=1}^{d} \Sigma_{j k} \frac{\partial^{2} f(x)}{\partial x_{j} \partial x_{k}}(x)+\sum_{j=1}^{d} \gamma_{j} \frac{\partial f}{\partial x_{j}}(x),
$$

sendo que as funções mensuráveis de Borel $\gamma_{j}, \Sigma_{j k}: \mathbb{R}^{d} \longrightarrow \mathbb{R}, 1 \leq j, k \leq d$. $O$ vetor $\gamma=\left\{\gamma_{j}\right\}_{j=1}^{d}$ é denominado coeficiente de t.d. (drift) e a matriz simétrica não-negativa definida $\Sigma=\left\{\Sigma_{j k}\right\}_{j, k=1}^{d}=\left\{\sigma \sigma_{j k}^{T}\right\}_{j, k=1}^{d}$ denominada coeficiente de difusão, para qualquer $x \in \mathbb{R}^{d}$.

Heuristicamente, o coeficiente de difusão $\gamma(x)$ pode ser interpretado como a velocidade local da trajetória do processo $X$ e $\sigma(x)$ a taxa de variação da matriz de covariância do vetor $X_{t}-x$, para pequenos valores $t>0$. Existem duas abordagens de estudo de difusões, uma puramente analítica e outra probabilística. Os precursores do estudo puramente analítico de difusões foram Komolgorov e Feller, que a partir 1930 buscaram, por meio da teoria de equações parciais diferenciais (determinísticas), e obtiveram a solução da seguinte equação diferencial

$$
\frac{\partial}{\partial t} f(y, t)=(\mathcal{A} f)(y, t)
$$

dado uma condição de contorno $f(y, 0)=y_{0}$. Neste contexto, os processos de difusão que satisfazem esta solução são denominados processo de difusão de Kolmogorov-Feller.

A abordagem probabilística, que se mostrou ser bem mais apropriada aos problemas de modelagem em finanças, foi inicialmente proposta por Lévy, sendo desenvolvida por Kiyosi Itô, a partir de 1940. Esta abordagem possibilita definir uma Difusão de Itô [Øksendal (2003), p.114]:

Definição 1.12 Processo de Difusão de Itô Um processo forte de Markov $X$ definido pelo mapeamento $X_{t}(\omega)=X(t, \omega):\left[0 ; \infty\left[\times \Omega \longrightarrow \mathbb{R}^{d}\right.\right.$, no espaço probabilístico $(\Omega, \mathcal{F}, \mathbb{P})$ é um processo de difusão de Itô, se e somente se satisfizer a equação diferencial estocástica:

$$
d X_{t}=\gamma\left(X_{t}\right) d t+\sigma\left(X_{t}\right) d B_{t}, t \geq \text { se } X_{s}=x
$$


sendo que $B_{t}$ é um $M B$ de dimensão d, e $\gamma: \mathbb{R}^{d} \longrightarrow \mathbb{R}^{d}, \sigma: \mathbb{R}^{d} \longrightarrow \mathbb{R}^{d \times d}$, sujeitas à

$$
|\gamma(x)-\gamma(y)|+|\sigma(x)-\sigma(y)| \leq k|x-y|, x, y \in \mathbb{R}^{d},
$$

sendo que $|\sigma|^{2}=\sum\left|\sigma_{i j}\right|^{2}$.

Uma forma equivalente de expressar a equação diferencial estocástica de Itô é:

$$
X_{t}=X_{0}+\int_{0}^{t} \sigma\left(X_{s}\right) d B_{s}+\int_{0}^{t} \gamma\left(X_{s}\right) d s .
$$

Conforme Dixit (1993), p.8, a diferença entre um processo de difusão e processo de difusão de Itô é que o primeiro depende apenas do estado corrente do processo, i.e., $X_{t}$, e o segundo, depende tando do estado corrente como do tempo. Apesar disto as duas definição são equivalentes [Øksendal (2003), p.123]. Ao considerar a restrição $f \in$ $\mathcal{C}^{2}\left(\mathbb{R}^{d}\right)$ na propriedade 2 da Definição 1.11 , tem-se que a equação diferencial estocástica proposta por Itô (1.18) é orientada pelo gerador infinitesimal (1.17). Desta maneira, neste estudo, o termo difusão refere-se, indiscriminadamente, tanto ao processo de difusão ou processo de difusão de Itô.

\subsection{Processo de Poisson}

O processo de Poisson é um processo estocástico que apresenta trajetórias descontínuas, sendo possível utilizá-lo como ponto de partida para processos com saltos mais complexos.

\subsubsection{Variáveis Estocásticas Exponenciais}

As propriedades das variáveis estocásticas exponenciais são importantes para definir processos de Markov com saltos. Uma v.e. $Y$ segue uma distribuição exponencial com parâmetro $\lambda>0$, se a sua função de densidade de probabilidade (f.d.p.) apresentar a seguinte forma

$$
\lambda \exp (-\lambda y) 1_{\{y \geq 0\}} .
$$

A função da distribuição de $Y$ é dada por

$$
\forall y \in\left[0 ; \infty\left[, \quad F_{Y}(y)=\mathbb{P}(Y \leq y)=1-\exp (-\lambda y)\right.\right.
$$


$F_{Y}$ é inversível e a sua função inversa é

$$
\forall y \in[0 ; 1], \quad F_{Y}^{-1}(y)=-\frac{1}{\lambda} \ln (1-y) .
$$

Uma conseqüência destes resultados é que se $U$ é uniformente distribuída em $[0 ; 1]$, então, $-\frac{1}{\lambda} \ln U$ é exponencialmente distribuída com parâmetro $\lambda$. Assim, por meio de distribuições uniformes, é computacionalmente possível simular uma distribuição exponencial.

A distribuição exponencial possui a propriedade: se $T$ é uma v.e. exponencial, então,

$$
\forall t, s>0, \mathbb{P}(T>t+s \mid T>t)=\frac{\int_{t+s}^{\infty} \lambda \exp (-\lambda y) d y}{\int_{t}^{\infty} \lambda \exp (-\lambda y) d y}=\mathbb{P}(T>s) .
$$

Em outras palavras, se interpretarmos $T$ como um tempo estocástico, a distribuição de $T-t$, i.e., a distribuição de $T>t$, é a mesma de $T$. Esta propriedade é denominada falta de memória. A distribuição exponencial é a única distribuição contínua com esta propriedade.

Proposição 1.2 Falta de Memória $\quad$ Seja $T \geq 0$ uma v.e. contínua, tal que

$$
\forall t, s>0, \mathbb{P}(T>t+s \mid T>t)=\mathbb{P}(T>s) .
$$

Então, T tem distribuição exponencial.

Prova Seja $g(t)=\mathbb{P}(T>t)$. Pela regra de Bayes temos $g$ é uma função multiplicativa:

$$
\forall t, s>0 g(t+s)=\mathbb{P}(T>t+s \mid T>t) \mathbb{P}(T>t)=g(s) g(t) .
$$

Dado que $1-g$ é a função da distribuição, $g$ deve ser decrescente e contínua à direita para que haja a convergência $\lim _{t \rightarrow \infty} 1-g(t)=1$. Estas duas características em conjunto indicam que a função $g$ é uma função exponencial decrescente, i.e. $g(t)=\exp (-\lambda t)$, para qualquer $\lambda>0$.

Sejam $\left(\tau_{i}, i=1,2, \ldots, n\right)$ variáveis estocásticas exponenciais independentes com parâmetros $\lambda$, e $T_{k}=\tau_{1}+\tau_{2}+\ldots+\tau_{k}$. Assim, $\left(T_{1}, \ldots, T_{n}\right)$ possui densidade conjunta de probabilidade definida em $\mathbb{R}^{n}$, dada por

$$
\lambda^{n} \exp \left(-\lambda t_{n}\right) 1_{\left\{0<t_{1}<\ldots<t_{n}\right\}}\left(t_{1}, \ldots, t_{n}\right) .
$$


A densidade de probabilidade de $T_{n}=\sum_{i=1}^{n} \tau_{i}$ é igual a

$$
p_{n}(t)=\lambda \exp (-\lambda t) \frac{(\lambda t)^{n-1}}{(n-1) !} 1_{[0 ; \infty[}(t) .
$$

A soma de variáveis estocásticas exponenciais i.i.d. guarda uma relação com o quantil de variáveis estocásticas uniformes. Seja $U_{1}, \ldots, U_{n}$ variáveis estocásticas uniformes independentes definidas em $[a ; b]$. É possível ordenar estas variáveis de maneira crescente, tal que a nova denominação será $\left(Y_{1}, \ldots, Y_{n}\right): Y_{n}=\max \left\{U_{i}, i=1, \ldots, n\right\}$, $Y_{n-1}=\max \left(\left\{U_{i}, i=1, \ldots, n\right\} /\left\{Y_{n}\right\}\right)$, etc. $Y_{1} \leq \ldots \leq Y_{n}$ são denominados de estatística de ordem de $U_{1}, \ldots, U_{n}$, e a função de densidade conjunta de probabilidade definida em $\mathbb{R}^{n}$ é dada por

$$
\frac{n !}{(b-a)^{n}} 1_{\left\{a<y_{1}<y_{1}, \ldots<y_{n}<b\right\}}\left(y_{1}, \ldots, y_{n}\right) .
$$

A f.d.p apresentada na equação (1.27) também é denominada Distribuição de Dirichlet e é representada por $D_{n}([a ; b])$. A partir da equação (1.25) é possível fazer aparecer a equação (1.26), como segue:

$$
\begin{aligned}
\lambda^{n} \exp \left(-\lambda t_{n}\right) 1_{\left\{0<t_{1}<\ldots<t_{n}\right\}}\left(t_{1}, \ldots, t_{n}\right)= & \lambda \exp \left(-\lambda t_{n}\right) \frac{\left(\lambda t_{n}\right)^{n-1}}{(n-1) !} 1_{\left\{t_{n} \geq 0\right\}} \times \\
& \frac{(n-1) !}{t_{n}^{n-1}} 1_{\left\{0<t_{1}<\ldots<t_{n}\right\}}\left(t_{1}, \ldots, t_{n}\right),
\end{aligned}
$$

que é simplesmente o produto da equação (1.26) com a equação $D_{n-1}\left(\left[0, t_{n}\right]\right)$. Isto conduz ao seguinte resultado:

Proposição 1.3 Sejam $\left(\tau_{i}, i=1, \ldots, n+1\right)$ variáveis estocásticas exponenciais independentes com parâmetro $\lambda e T_{k}=\tau_{1}+\ldots+\tau_{k}$. Então,

1. Af.d.p. de $\left(T_{1}, \ldots, T_{n}\right)$, dado que é conhecido $T_{n+1}=t$, é $D_{n}([0 ; t])$.

2. O vetor $\left(\frac{T_{1}}{T_{n+1}}, \ldots, \frac{T_{n}}{T_{n+1}}\right)$ é independente de $T_{n+1}$ e tem f.d.p. $D_{n}([0 ; 1])$.

Esta proposição é de interesse prático, pois ela permite a simulação da densidade conjunta de probabilidade de $\left(T_{1}, \ldots, T_{n}\right)$ através de variáveis estocásticas uniformes independentes. 
Proposição 1.4 Sejam $U_{1}, \ldots, U_{n}$ variáveis estocásticas independentes, uniformemente distribuídas no intervalo $[0 ; 1], U_{(1)} \leq \ldots \leq U_{(n)}$ as correspondentes estatísticas de ordem, e $p_{n}(\cdot)$ uma v.e. independente com f.d.p. igual à função apresenta pela equação (1.26). Assim, as variáveis estocásticas

$$
p_{n}\left(U_{(1)}\right), p_{n}\left(U_{(2)}-U_{(1)}\right), p_{n}\left(U_{(3)}-U_{(2)}\right), \ldots, p_{n}\left(U_{(n)}-U_{(n-1)}\right)
$$

formam uma seqüência de variáveis exponenciais independentes de parâmetro $\lambda$.

\subsubsection{A Distribuição de Poisson}

Uma v.e. $N$ de valores naturais segue uma distribuição de Poisson com parâmetro $\lambda$ se

$$
\forall n \in \mathbb{N}, \mathbb{P}(N=n)=\exp (-\lambda) \frac{\lambda^{n}}{n !}
$$

A distribuição de Poisson possui m.g.f. bem definida, dada por:

$$
M(u)=\exp [\lambda(\exp (u)-1)]
$$

Existe uma relação bem próxima entre a distribuição de Poisson e a soma de variáveis exponenciais independentes.

Proposição 1.5 Se $\left\{\tau_{i}\right\}_{i \geq 1}$ são variáveis estocásticas exponenciais de parâmetro $\lambda$, então, para qualquer $t>0$ a v.e.

$$
N_{t}=\inf \left\{n \geq 1, \sum_{i=1}^{n} \tau_{i}>t\right\}
$$

segue uma distribuição de Poisson de parâmetro $\lambda t$ :

$$
\forall n \in \mathbb{N}, \mathbb{P}\left(N_{t}=n\right)=\exp \{-\lambda t\} \frac{(\lambda t)^{n}}{n !} .
$$

Prova Seja $T_{k}=\sum_{i-1}^{k} \tau_{i}, \forall k$. A densidade de $\left(T_{1}, \ldots, T_{n}\right)$ é dado por

$$
\lambda^{n} \exp \left(-\lambda t_{n}\right) 1_{\left\{0<t_{1}<\ldots<t_{n}\right\}}\left(t_{1}, \ldots, t_{n}\right) .
$$


Como a $\mathbb{P}\left(N_{t}=n\right)=\mathbb{P}\left(T_{n} \leq t<T_{n+1}\right)$, então, tem-se que:

$$
\begin{aligned}
\mathbb{P}\left(N_{t}=n\right) & =\int_{0<t_{1}<\ldots<t_{n}<t<t_{n+1}} \lambda^{n+1} \exp \left(-\lambda t_{n+1}\right) d t_{1} \ldots d t_{n} d t_{n+1} \\
& =\int_{0<t_{1}<\ldots<t_{n}<t}\left(\int_{t}^{\infty} \lambda^{n+1} \exp \left(-\lambda t_{n+1}\right) d t_{n+1}\right) d t_{1} \ldots d t_{n} \\
& =\lambda^{n} \exp (-\lambda t) \int_{0}^{t}\left\{\int_{0}^{t_{n}} \cdots\left[\int_{0}^{t_{3}}\left(\int_{0}^{t_{2}} d t_{1}\right) d t_{2}\right] \ldots d t_{n-1}\right\} d t_{n} \\
& =\lambda^{n} \exp (-\lambda t) \int_{0}^{t}\left[\int_{0}^{t_{n}} \cdots\left(\int_{0}^{t_{j+1}} \frac{1}{(j-1) !} t_{j}^{j-1} d t_{j}\right) \ldots d t_{n-1}\right] d t_{n} \\
& =\exp (-\lambda t) \frac{(\lambda t)^{n}}{n !} .
\end{aligned}
$$

Uma propriedade interessante da distribuição de Poisson é a sua estabilidade sob convoluções, i.e., se $Y_{1}$ e $Y_{2}$ são variáveis estocásticas de Poisson independentes, com parâmetros $\lambda_{1}$ e $\lambda_{2}$, então, $Y_{1}+Y_{2}$ também possui densidade de Poisson com parâmetro $\lambda_{1}+\lambda_{2}$. Esta propriedade pode ser facilmente deduzida através da m.g.f. da distribuição de Poisson, pois $M_{Y_{1}+Y_{2}}=M_{Y_{1}} M_{Y_{2}}$. Particularmente, isto indica a seguinte conseqüência: para qualquer inteiro $n$, uma v.e. de Poisson $Y$ com parâmetro $\lambda$ pode ser expressa como sendo a soma de $n$ variáveis estocásticas de Poisson independentes $Y_{i}$ com parâmetro $\lambda / n$. Esta propriedade é chamada de divisibilidade infinita, e pode ser interpretada como a capacidade da v.e. de Poisson de ser dividida por um número arbitrário de variáveis estocásticas i.i.d.

\subsubsection{Propriedades do Processo de Poisson}

O processo de Poisson pode ser definido como:

Definição 1.13 Processo de Poisson Seja $\left\{\tau_{i}\right\}_{i \geq 1}$ uma seqüencia de variáveis estocásticas exponenciais com parâmetro $\lambda$ e $T_{n}=\sum_{i=1}^{n} \tau_{i}$. O processo $\left\{N_{t}\right\}_{t \geq 0}$, definido como

$$
N_{t}=\sum_{n \geq 1} 1_{\left\{t \geq T_{n}\right\}}
$$

é um processo de Poisson de intensidade igual a $\lambda$. 
Desta maneira, o processo Poisson deve ser entendido como um processo de contagem, pois ele conta a quantidade de tempos estocásticos $\left\{T_{n}\right\}$ que ocorrem entre $0 \mathrm{e} t$, sendo que $\left\{T_{n}-T_{n-1}\right\}_{n \geq 1}$ é um seqüência i.i.d. de variáveis estocásticas exponenciais.

Proposição 1.6 Se $\left\{N_{t}\right\}_{t \geq 0}$ é um processo Poisson, então, valem as seguintes propriedades:

1. Para qualquer $t>0, N_{t}$ é quase-certamente finito.

2. Para qualquer $\omega$, o caminho observado $t \longrightarrow N_{t}(\omega)$ é constante por trechos $e$ cresce por saltos de tamanho 1.

3. O caminho observado $t \longmapsto N_{t}$ é contínuo à direita com limite à esquerda (cadlag).

4. Para qualquer $t>0, \mathbb{P}\left[N_{t-}=N_{t}\right]=1$, ou de maneira equivalente, $\mathbb{P}\left[N_{t-} \neq N_{t}\right]=0$.

5. $\left\{N_{t}\right\}$ é contínuo em probabilidade:

$$
\forall t>0, N_{s} \underset{s \rightarrow t}{\stackrel{\mathbb{P}}{\longrightarrow}} N_{t} .
$$

6. Para qualquer $t>0, N_{t}$ tem distribuição de Poisson de parâmetro $\lambda t$ :

$$
\forall n \in \mathbb{N}, \mathbb{P}\left(N_{t}=n\right)=\exp (-\lambda t) \frac{(\lambda t)^{n}}{n !} .
$$

7. A função característica de $N_{t}$ é dado por

$$
\mathbb{E}\left[\exp \left(i u N_{t}\right)\right]=\exp \{\lambda t(\exp (i u)-1)\}, \forall u \in \mathbb{R}
$$

8. $\left\{N_{t}\right\}$ apresenta incrementos independentes: para qualquer $t_{1}<\ldots<t_{n}$,

$$
N_{t_{n}}-N_{t_{n-1}}, \ldots, N_{t_{2}}-N_{t_{1}}, N_{t_{1}}
$$

são variáveis estocásticas independentes.

9. Os incrementos de $\left\{N_{t}\right\}$ são homogêneos [propriedade 2 da Definição 1.9]: para qualquer $t>s, N_{t}-N_{s}$ tem a mesma distribuição de $N_{t-s}$.

10. $\left\{N_{t}\right\}$ possui a propriedade de Markov [propriedade 3 da Definição 1.8]:

$$
\forall t>s, \mathbb{E}\left[f\left(N_{t}\right) \mid N_{u}, u \leq s\right]=\mathbb{E}\left[f\left(N_{t}\right) \mid N_{s}\right] .
$$




\section{Prova}

1. Seja $\Omega_{1}=\left\{\omega \in \Omega, \frac{T_{n}}{n}(\omega) \longrightarrow \frac{1}{\lambda}\right\}$. Pela Lei dos Grandes Números, $\frac{T_{n}}{n} \longrightarrow \frac{1}{\lambda}$ ocorre com probabilidade 1 , o que implica em $\mathbb{P}\left(\Omega_{1}\right)=1$. Para qualquer $\omega \in \Omega_{1}$, $T_{n}(\omega) \longrightarrow \infty$, tem-se que

$$
\forall \omega \in \Omega_{1}, \exists n_{0}(\omega) \geq 1, T_{n}(\omega)>t, \forall n \geq n_{0} .
$$

Como, $\mathbb{P}\left(N_{t}<\infty\right)=\mathbb{P}\left(\Omega_{1}\right)=1$, então, o número de termos na soma em (1.31) é quase-certamente finito.

2. Pela expressão (1.31) fica claro que $N_{t}$ é constante para cada intervalo $] T_{n} ; T_{n+1}[\mathrm{e}$ que cresce unitariamente em $T_{n}$. Como a quantidade de saltos a cada intervalo $[0 ; t]$ é quase-certamente finito, então, a propriedade 2 é garantida.

3. A propriedade cadlag pode ser verificada ao comparar a Definição 1.13 do processo de Poisson, i.e.,

$$
N_{t}=\sum_{n \geq 1} 1_{t \in\left[T_{n} ; \infty[\right.}
$$

com o exemplo de função cadlag apresentada na equação (1.3).

4. Para um $\omega \in \Omega$, os pontos de descontinuidades de $N_{t}(\omega)$ é $\left\{T_{n}(\omega), n \geq 1\right\}$. Como as distribuições contínuas atribuem probabilidade zero para qualquer ponto (medida difusa), para um dado $t, \mathbb{P}\left(t \in\left\{T_{n}(\omega), n \geq 1\right\}\right)=0$. Assim, com probabilidade $1, t$ não é um ponto descontínuo, i.e., $N_{t-}=N_{t}$ com probabilidade 1 .

5. Uma conseqüência da prova acima é: uma vez garantida a convergência quasecerta, então, a convergência em probabilidade também estará garantida (vide Seção D.2).

6. Esta prova foi efetuada na Proposição 1.2.

7. Esta é uma conseqüência lógica da propriedade 6.

8. Seja $0<t_{1}<\cdots<t_{n}$, tem-se que

$$
\mathbb{P}\left(N_{t_{1}}=k_{1}, N_{t_{2}}-N_{t_{1}}=k_{2}, \ldots, N_{t_{n}}-N_{t_{n-1}}=k_{n}\right)
$$


Ao definir $j_{n}=\sum_{i \leq n} k_{i}$ para $i \geq 1$, então, a probabilidade acima pode ser reescrita como:

$$
\mathbb{P}\left(T_{j_{1}} \leq t_{1}<T_{j_{1}+1}, T_{j_{2}} \leq t_{2}<T_{j_{2}+1}, \ldots, T_{j_{n}} \leq t_{n}<T_{j_{n}+1}\right) .
$$

Condicionada em $T_{j_{n}} \leq t_{n}<T_{j_{n}+1},\left(T_{1}, T_{2}, \ldots, T_{j_{n}}\right)$ é distribuída como as estatísticas de ordem $j_{n}$ variáveis estocásticas $U_{1}, U_{2}, \ldots, U_{j_{n}}$ uniformemente distribuídas no intervalo $\left[0 ; t_{n}\right]$. A probabilidade condicionada

$$
\mathbb{P}\left(T_{j_{1}} \leq t_{1}<T_{j_{1}+1}, T_{j_{2}} \leq t_{2}<T_{j_{2}+1}, \ldots \mid T_{j_{n}} \leq t_{n}<T_{j_{n}+1}\right)
$$

é igual a probabilidade, $\left(U_{(1)}, \ldots, U_{\left(k_{1}\right)}\right) \in\left[0 ; t_{1}\right]$, tal que, $\left(U_{\left(k_{1}\right)}, \ldots, U_{\left(k_{2}\right)}\right) \in$ ]$\left.\left.\left.t_{1} ; t_{2}\right], \ldots,\left(U_{\left(k_{n-1}\right)}, \ldots, U_{\left(k_{n}\right)}\right) \in\right] t_{n-1} ; t_{n}\right]$. A probabilidade de $U_{k}$ pertencer a $\left[t_{i-1} ; t_{i}\right]$ é igual a $\left(t_{i}, t_{i-1}\right) / t_{n}$. Ao aplicar o resultado (1.27), tem-se que a distribuição condicionada (1.37) é igual a

$$
\begin{aligned}
& \int_{0<u_{(1)}<\cdots<u_{\left(k_{1}\right)}<t_{1}<u_{\left(k_{1}+1\right)} \cdots<u_{\left(k_{n}\right)}<t_{n}}\left(\frac{j_{n} !}{t_{n}^{j_{n}}}\right) d u_{(1)} d u_{(2)} \cdots d u_{(n)} \\
& =\frac{j_{n} !}{t_{n}^{j_{n}}} \int_{t_{n-1}}^{t_{n}} \int_{t_{n-1}}^{u_{\left(k_{n}\right)}} \cdots \int_{t_{1}}^{u_{\left(k_{1}+2\right)}} \int_{0}^{t_{1}} \cdots \int_{0}^{u_{(2)}} d u_{(1)} \cdots d u_{\left(k_{1}\right)} d u_{\left(k_{1}+1\right)} \cdots d u_{(n-1)} d u_{(n)} \\
& =\frac{j_{n} !}{t_{n}^{j_{n}}} \int_{t_{n-1}}^{t_{n}} \int_{t_{n-1}}^{u_{\left(k_{n}\right)}} \cdots \int_{t_{1}}^{u_{\left(k_{1}+2\right)}}\left(\frac{t_{1}^{k_{1}}}{k_{1} !}\right) d u_{\left(k_{1}+1\right)} \cdots d u_{(n-1)} d u_{(n)} \\
& =\frac{j_{n} !}{t_{n}^{j_{n}}} \frac{t_{1}^{k_{1}}}{k_{1} !} \prod_{i=2}^{n} \frac{\left(t_{i}-t_{i-1}\right)^{k_{i}}}{k_{i} !} \text {. }
\end{aligned}
$$

Para obter a probabilidade desejada (1.36) é necessário multiplicar a probabilidade condicionada (1.38) pela probabilidade $\mathbb{P}\left(N_{t_{n}}=j_{n}\right)=\mathbb{P}\left(T_{j_{n}} \leq t_{n}<T_{j_{n}+1}\right)$ que, conforme Proposição 1.5, possui distribuição de Poisson com intensidade $\lambda t_{n}$. Após as simplificações tem-se:

$$
\lambda^{j_{n}} \exp \left(-\lambda t_{n}\right) \frac{t_{1}^{k_{1}}}{k_{1} !} \prod_{i=2}^{n} \frac{\left(t_{i}-t_{i-1}\right)^{k_{i}}}{k_{i} !} .
$$

Por último, ao substituir $j_{n}=k_{1}+k_{2}+\cdots+k_{n}$, é possível fatorar a probabilidade obtida em (1.39) em $n$ termos:

$$
\frac{\left(\lambda t_{1}\right)^{k_{1}} \exp \left(-\lambda t_{1}\right)}{k_{1} !} \prod_{i=2}^{n} \exp \left(-\left[\lambda\left(t_{i}-t_{i-1}\right)\right]\right) \frac{\left[\lambda\left(t_{i}-t_{i-1}\right)\right]^{k_{i}}}{k_{i} !} .
$$

Como a distribuição conjunta dos incrementos é fatorável nas distribuições individuais de cada incremento, pela própria definição de independência (C.9) temos que os incrementos são distribuídos independentemente. 
9. Pela propriedade 8 cada um dos incrementos $N_{t_{i}}-N_{t_{i-1}}$ possui função de densidade de Poisson com intensidade igual a $\lambda\left(t_{i}-t_{i-1}\right)$, i.e., igual a $N_{t_{i}-t_{i-1}}$. Assim, os incrementos são homogêneos.

10. A propriedade de Markov provém da independência dos incrementos:

$$
\begin{aligned}
\mathbb{E}\left[f\left(N_{t}\right) \mid N_{u}, u \leq s\right] & =\mathbb{E}\left[f\left(N_{t}-N_{s}+N_{s}\right) \mid N_{u}, u \leq s\right], \forall t>s \\
& =\mathbb{E}\left[f\left(N_{t}-N_{s}+N_{s}\right) \mid N_{s}\right] .
\end{aligned}
$$

Como $N_{t}-N_{s}$ é independente de $N_{u} u \leq s$, então, a propriedade 10 é garantida.

As propriedades 2, 3 e 4 de um processo de Poisson parecem ser paradoxais. De uma lado, tem-se que o caminho percorrido pelo processo de Poisson é descontínuo com probabilidade igual a 1 (de fato o processo cresce em saltos), por outro lado, a qualquer ponto $t$, a função que gera o caminho é contínua com probabilidade 1. Esta característica aparece um muitos processos com saltos utilizados na modelagem de mercados financeiros. Isto ocorre pelo fato que os pontos descontínuos do processo formam um conjunto de medidas difusas (vide definição na p.197).

A continuidade à direita (propriedade cadlag) de um processo de Poisson não é de fato uma propriedade, pois $N_{t}$ foi inicialmente definido de tal maneira que no ponto de descontinuidade $N_{t}=N_{t+}$, i.e., foi escolhido previamente a versão cadlag do processo de Poisson. Uma outra escolha seria o processo contínuo à esquerda, ou seja,

$$
N_{t}^{\prime}=\sum_{n \geq 0} 1_{\left\{t>T_{n}\right\}}
$$

A diferença entre $N_{t}$ e $N_{t}^{\prime}$ é que os valores de $N_{t}^{\prime}$ são previsíveis em intervalos de tempo futuro pequeno, pois $N_{s}^{\prime} \longrightarrow N_{t}$ à medida que $s \longrightarrow t$, para $s<t$, enquanto que, $N_{t}$ é imprevisível dado a sua trajetória passada. Os saltos de $N_{t}$ podem ser interpretados como eventos-surpresa, e correspondem à motivação inicial de incluir saltos nos modelos e preços dinâmicos. Assim, é interessante escolher sempre a versão cadlag $\left\{N_{t}\right\}_{t \geq 0}$ dos processos de Poisson. Caso fosse adotado outro processo com saltos para modelar preços, a escolha seria sempre pela versão cadlag destes processos.

A Figura 1.1 apresenta dois caminhos de um processo de Poisson. 


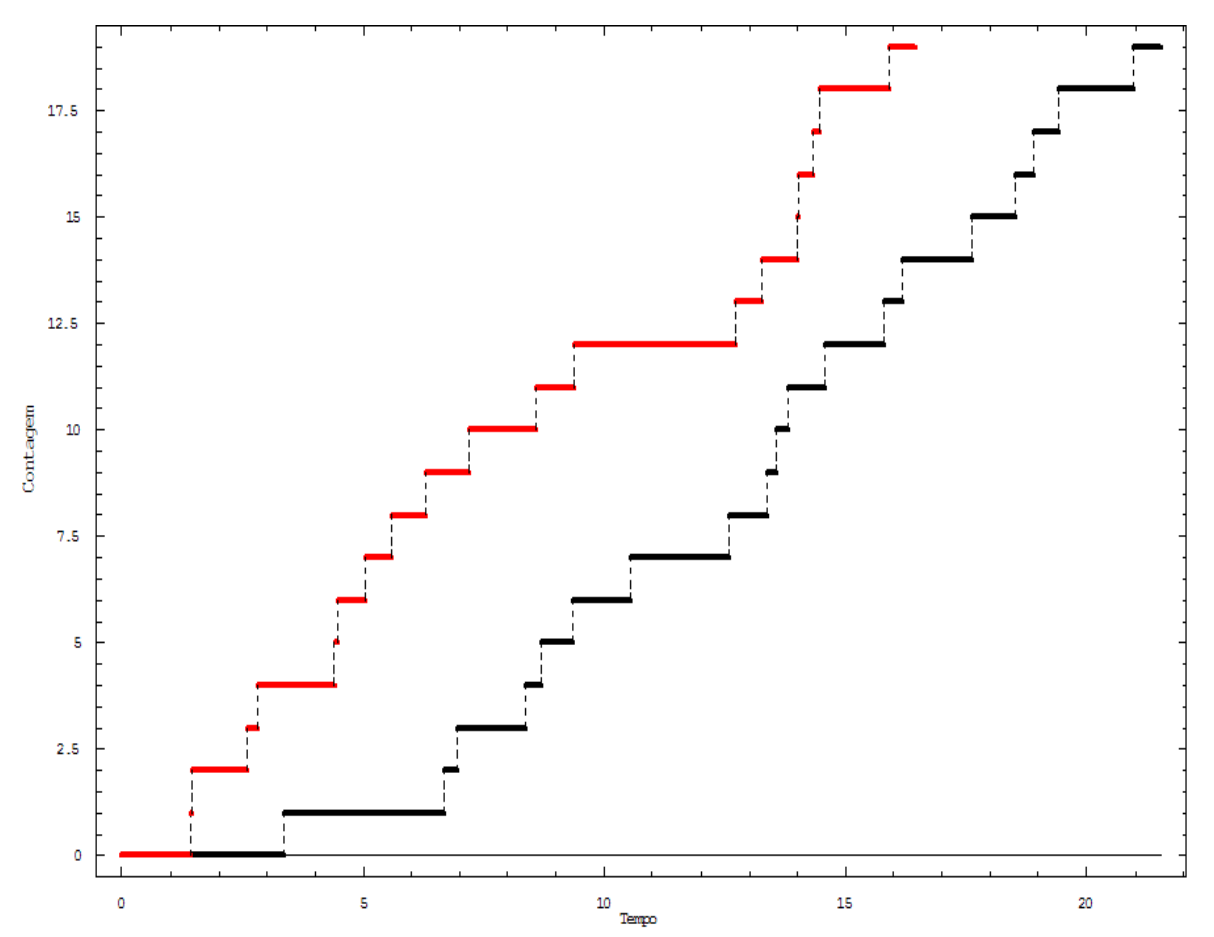

Figura 1.1. Simulação de dois processos de Poisson pelo mesmo p.g.d. com $\lambda=1$.

Os processos de Poisson apresentam propriedades muito úteis. A primeira delas é a propriedade de sobreposição de incrementos do processo de Poisson, que também é um processo de Poisson.

Proposição 1.7 Soma de Processos de Poisson Independentes $\quad$ Se $\left\{N_{t}^{1}\right\}_{t \geq 0}$ $e\left\{N_{t}^{2}\right\}_{t \geq 0}$ são processos de Poisson independentes com intensidades $\lambda_{1} e \lambda_{2}$, então, $\left\{N_{t}^{1}+N_{t}^{2}\right\}_{t \geq 0}$ é um processo de Poisson com intensidade igual a $\lambda_{1}+\lambda_{2}$.

Outra propriedade interessante dos processos de Poisson é a propriedade de redução. Seja $\left\{N_{t}\right\}_{t \geq 0}$ um processo de Poisson com intensidade $\lambda$ e estabeleça um processo $X_{t}$ pela redução de $N_{t}$, i.e., pegue todos os saltos $\left(T_{n}, n \geq 1\right)$ referentes à $\left\{N_{t}\right\}_{t \geq 0}$ e mantenha-os no processo com $0<p<1$ ou retire-os do processo com probabilidade $1-p$, independentemente da decisão anterior. Ordene os pontos que não foram excluídos: $T_{1}^{\prime}, \ldots, T_{n}^{\prime}, \ldots$ e definida

$$
X_{t}=\sum_{n \geq 1} 1_{\left\{T_{n}^{\prime} \geq t\right\}}
$$


Desta maneira, o processo $X$ também será um processo de Poisson com intensidade $p \lambda$. Outra forma de obter este resultado é através do seguinte procedimento: se a chegada $T_{n}$ de cada evento no processo de Poisson $\left\{N_{t}\right\}_{t \geq 0}$ de intensidade $\lambda$ é marcado com probabilidade $p$, independemente de cada evento, então, o processo marcado é novamente um processo de Poisson com intensidade igual a $\lambda_{X}=p \lambda$.

\subsubsection{Processo de Poisson Compensado}

Defina uma versão centralizada do processo de Poisson $\left\{N_{t}\right\}_{t \geq 0}$ como

$$
\tilde{N}_{t}=N_{t}-\lambda t
$$

$\tilde{N}_{t}$ seguirá uma versão centralizada da distribuição de Poisson com função característica:

$$
\Phi_{\tilde{N}_{t}}(z)=\exp [\lambda t(\exp \{i z\}-1-i z)]
$$

Como o processo de Poisson, $\tilde{N}_{t}$ é também independente por incrementos e é fácil demonstrar que:

$$
\begin{aligned}
\mathbb{E}\left[N_{t} \mid N_{s}, s \leq t\right] & =\mathbb{E}\left[N_{t}-N_{s}+N_{s} \mid N_{s}, s \leq t\right] \\
& =\mathbb{E}\left[N_{t}-N_{s}\right]+N_{s}=\lambda(t-s)+N_{s},
\end{aligned}
$$

então, $\left\{\tilde{N}_{t}\right\}_{t \geq 0}$ possui a propriedade martingale:

$$
\forall t>s, \mathbb{E}\left[\tilde{N}_{t} \mid \tilde{N}_{s}, s \leq t\right]=\tilde{N}_{s}
$$

Assim, $\left\{\tilde{N}_{t}\right\}_{t \geq 0}$ é denominado processo de Poisson compensado e a expressão determinística $\{\lambda t\}_{t \geq 0}$ é denominado compensador do processo $\left\{N_{t}\right\}_{t \geq 0}$. O compensador é a quantidade esperada (determinística) que deve ser subtraída do processo $\left\{N_{t}\right\}$ para que seja um processo martingale. A Figura 1.2 apresenta caminhos do processo de Poisson.

Cabe ressaltar que, o processo de Poisson compensado não é mais um processo de valores inteiros, pois diferentemente do processo de Poisson, ele não é um processo de contagem. A versão reescalonada $\tilde{N}_{t} / \lambda$ tem os primeiros momentos iguais à um processo de Wiener:

$$
\mathbb{E}\left[\frac{\tilde{N}_{t}}{\lambda}\right]=0 \quad \mathbb{V}\left[\frac{\tilde{N}_{t}}{\lambda}\right]=t
$$




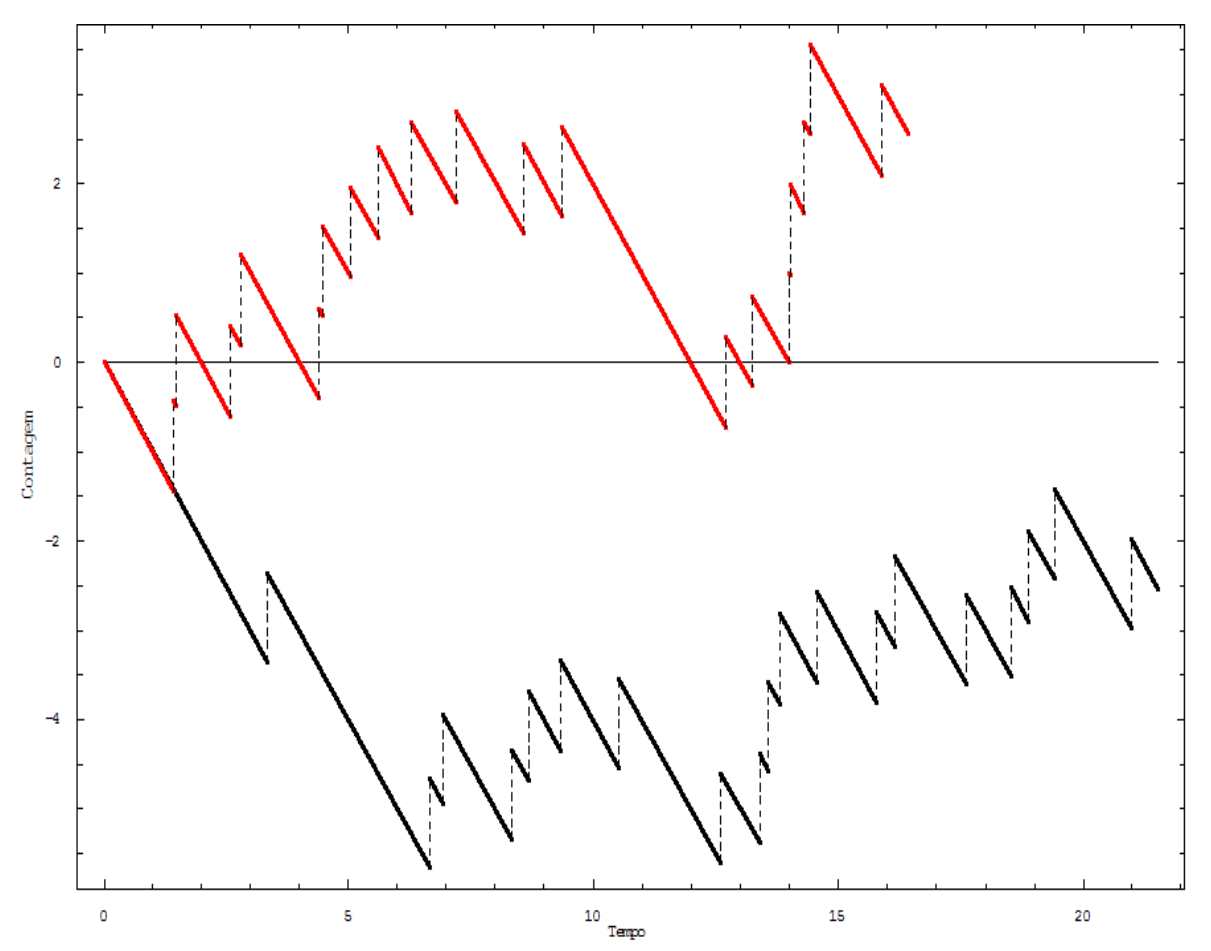

Figura 1.2. Processos de Poisson simulados na Figura 1.1 sem a tendência determinística.

Ambas as figuras parecem similares, e não é por coincidência, pois quando a intensidade dos saltos aumenta, o processo de Poisson compensado reescalonado converge para a distribuição do Processo de Wiener:

$$
\left\{\frac{\tilde{N}_{t}}{\lambda}\right\}_{t \in[0 ; T]} \stackrel{\lambda \rightarrow \infty}{\Longrightarrow}\left\{W_{t}\right\}_{t \in[0 ; T]} .
$$

Este resultado é uma conseqüência do Princípio da Invariância de Donsker [Kallenberg (2002), Teorema 14.9, p.275] que pode ser visto como um Teorema do Limite Central definido em $\Omega=\boldsymbol{D}([0 ; T])$.

\subsubsection{Processo de Contagem}

O processo Poisson $N_{t}$ conta a quantidade de tempos estocásticos $\left\{T_{n}\right\}_{n \geq 1}$ que ocorrem em $[0 ; t]$ onde os tempos estocásticos $T_{n}$ são somas parciais de uma seqüência de variáveis estocásticas i.i.d. exponenciais. De uma maneira geral, dado uma seqüência de tempos estocásticos $\left\{T_{n}\right\}_{n \geq 1} \operatorname{com} \mathbb{P}\left[T_{n} \longrightarrow \infty\right]=1$, é possível definir um 
processo de contagem $\left\{X_{t}\right\}_{t \geq 0}$ associado a estes tempos estocásticos como

$$
X_{t}=\sum_{n \geq 1} 1_{\left\{t \geq T_{n}\right\}}=\inf \left\{n \geq 1: T_{n} \geq t\right\} .
$$

$X_{t}$ é simplesmente o número de tempos estocásticos $\left\{T_{n}\right\}_{n \geq 1}$ que ocorre no intervalo $[0 ; t]$. A condição $\mathbb{P}\left(T_{n} \longrightarrow \infty\right)=1$ garante que, com probabilidade $1, X_{t}$ é bem definida (finita) para qualquer $t \geq 0$. Como um processo de Poisson, $\left\{X_{t}\right\}_{t \geq 0}$ é um processo cadlag de trajetória constante por partes, ou seja, o seu caminho estocástico move-se por saltos de tamanho igual a 1 .

Se os tempos estocásticos $\left\{T_{n}\right\}$ são construídos como a soma parcial de uma seqüência de variáveis estocásticas i.i.d. exponenciais, então, $X$ é um processo de Poisson. Para processo de contagem mais genéricos, a seqüência de tempos estocásticos $\left\{T_{n}\right\}$ pode ter uma distribuição com dependência entre as variáveis. O seguinte lema mostra que apenas os processos com incrementos estacionários independentes são processos de Poisson.

Lema 1.1 Seja $\left\{X_{t}\right\}$ um processo de contagem com incrementos independentes e estacionários. Então, $\left\{X_{t}\right\}$ é um processo de Poisson.

Prova Seja $T_{k}=\inf \left\{t \geq 0: X_{t} \geq k\right\}$. Considere $n \geq 1, Y_{n}=T_{n}-T_{n-1}$. Dado que os incrementos são independentes, é possível provar que $Y_{1}$ possui distribuição exponencial.

$$
\begin{aligned}
\mathbb{P}\left[Y_{1}>t+s \mid Y_{1}>t\right] & =\mathbb{P}\left[X_{t+s}=0 \mid X_{t}=0\right] \\
& =\mathbb{P}\left[X_{t+s}-X_{t}=0 \mid X_{t}=0\right]=\mathbb{P}\left[X_{t+s}-X_{t}=0\right] \\
& =\mathbb{P}\left[X_{s}=0\right]=\mathbb{P}\left[Y_{1}>s\right]
\end{aligned}
$$

Isto significa que $Y_{1}$ possui a propriedade de falta de memória. Pela Proposição 1.2, a falta de memória implica que $Y_{1}$ é uma v.e. exponencial. O próximo passo é provar que $\left\{X_{t+Y_{1}}-X_{Y_{1}}\right\}_{t \geq 0}$ é independente de $Y_{1}$ e possui distribuição igual a $\left\{X_{t}\right\}_{t \geq 0}$. Sabe-se $Y_{1}$ é tempo de parada não-antecipável do processo $\left\{X_{t}\right\}_{t \geq 0}$, pois o evento $\left\{Y_{1} \leq t\right\}=$ $\left\{X_{t} \geq 1\right\}$ não depende da trajetória de $X$ depois de $t$.

Seja $f(t)=\mathbb{E}\left[\exp \left(i u . X_{t}\right)\right]$ para qualquer $u$ fixo. Dado que $X$ é independente e 
estacionário por incrementos, então para qualquer $s, t>0, f(s+t)=f(s) f(t)$, e

$$
M_{t}=\frac{\exp \left(i u X_{t}\right)}{f(t)}
$$

é uma martingale. Esta propriedade é obtida pela transformada de Esscher [Gerber \& Shiu (1994a) e Gerber \& Shiu (1994b)].

Seja $Y_{1}^{n}=n \wedge Y_{1}$. Dado que $Y_{1}^{n}$ é um tempo de parada com limite superior, e que

$$
f(-t) \exp \left\{i u\left(X_{Y_{1}^{n}+t}-X_{Y_{1}^{n}}\right)\right\}
$$

é uma martingale, então, pela Proposição 1.1 (Teorema da Amostragem Opcional de Doob) tem-se que

$$
\begin{aligned}
& \mathbb{E}\left[\exp \left\{i u\left(X_{Y_{1}^{n}+t}-X_{Y_{1}^{n}}\right)+i v Y_{1}^{n}\right\} \mid \mathcal{F}_{Y_{1}^{n}}\right] \\
= & \mathbb{E}\left[f(-t) \exp \left\{i u\left(X_{Y_{1}^{n}+t}-X_{Y_{1}^{n}}\right)\right\} \mid \mathcal{F}_{Y_{1}^{n}}\right] f(t) \exp \left\{i v Y_{1}^{n}\right\} \\
= & {\left[f(0) \exp \left\{i u\left(X_{Y_{1}^{n}}-X_{Y_{1}^{n}}\right)\right\}\right] f(t) \exp \left\{i v Y_{1}^{n}\right\} } \\
= & f(t) \exp \left\{i v Y_{1}^{n}\right\} .
\end{aligned}
$$

Ao aplicar o operador esperança de ambos os lados da equação (1.48), pela Lei das Esperanças Iteradas, obtém-se a esperança não condicionada a filtragem $\mathcal{F}_{Y_{1}^{n}}$, i.e.,

$$
\begin{aligned}
\mathbb{E}\left[\mathbb{E}\left[\exp \left\{i u\left(X_{Y_{1}^{n}+t}-X_{Y_{1}^{n}}\right)+i v Y_{1}^{n}\right\} \mid \mathcal{F}_{Y_{1}^{n}}\right]\right] & =\mathbb{E}\left[f(t) \exp \left\{i v Y_{1}^{n}\right\}\right] \\
\mathbb{E}\left[\exp \left\{i u\left(X_{Y_{1}^{n}+t}-X_{Y_{1}^{n}}\right)+i v Y_{1}^{n}\right\}\right] & =f(t) \mathbb{E}\left[\exp \left\{i v Y_{1}^{n}\right\}\right] \\
\mathbb{E}\left[\exp \left\{i u\left(X_{Y_{1}^{n}+t}-X_{Y_{1}^{n}}\right)+i v Y_{1}^{n}\right\}\right] & =\mathbb{E}\left[\exp \left\{i u X_{t}\right\}\right] \mathbb{E}\left[\exp \left\{i v Y_{1}^{n}\right\}\right]
\end{aligned}
$$

Dado que os incrementos são estacionários, é possível calcular o limite $n \longrightarrow \infty$, sendo que o resultado converge para um número finito:

$$
\mathbb{E}\left[\exp \left\{i u\left(X_{Y_{1}+t}-X_{Y_{1}}\right)+i v Y_{1}\right\}\right]=\mathbb{E}\left[\exp \left\{i u X_{t}\right\}\right] \mathbb{E}\left[\exp \left\{i v Y_{1}\right\}\right]
$$

Este resultado (1.49) sugere que o processo $\left\{X_{Y_{1}+t}-X_{Y_{1}}\right\}_{t \geq 0}$ é independente de $Y_{1}$ (falta de memória dos incrementos em relação à filtragem) e é estacionário por incrementos pois tem distribuição igual de $\left\{X_{t}\right\}_{t \geq 0}$. Ao substituir $t$ por $Y_{2}$, é fácil verificar pelo resultado apresentado acima, que $Y_{2}$ é exponencialmente distribuída e independente de $Y_{1}$. Ao repetir a estrutura de raciocínio é possível verificar que $Y_{3}$ é também exponencialmente distribuída e independente de $Y_{1}$ e $Y_{2}$. Assim por indução finita:

$$
\mathbb{E}\left[Y_{i} \mid Y_{i-1}, \cdots, Y_{1}\right]=\mathbb{E}\left[Y_{i}\right], i>2 .
$$


Pela Proposição 1.5 combinado com o resultado (1.50) o processo de contagem $\left\{X_{t}\right\}_{t \geq 0}$ é um processo de Poisson.

\subsection{Medidas Estocásticas e Processos Pontuais}

O Processo de Poisson $N=\left\{N_{t}\right\}_{t \geq 0}$ [Definição 1.13] pode ser definido como um processo de contagem, sendo que $T_{1}, T_{2}, \ldots$ é a seqüência temporal dos saltos em $N$, e $N_{t}$ é a quantidade de saltos no intervalo de tempo $[0 ; t]$, i.e.,

$$
N_{t}=\#\left\{i \geq 1, T_{i} \in[0 ; t]\right\}
$$

De maneira análoga, para $t>s$ :

$$
\left.\left.N_{t}-N_{s}=\#\left\{i \geq 1, T_{i} \in\right] s ; t\right]\right\} .
$$

A seqüência temporal dos saltos $T_{1}, T_{2}, \ldots$ forma a configuração estocástica de pontos em $\left[0 ; \infty\left[\right.\right.$ e, a v.e. de Poisson $N_{t}$ conta a quantidade de pontos no intervalo $[0 ; t]$. O procedimento de contagem define uma medida $M$ em $[0 ; \infty[:$ para qualquer conjunto mensurável $A \subset \mathbb{R}^{+}$, seja

$$
M(\omega, A)=\#\left\{i \geq 1, T_{i}(\omega) \in A\right\}
$$

Desta maneira, $M(\omega, \cdot)$ é uma medida positiva de valores inteiros e $\mathbb{P}(M(A)<\infty)=$ 1, para qualquer conjunto fechado $A$. Cabe ressaltar que, a medida $M(\omega, \cdot)$ depende de $\omega$, i.e., ela é uma medida estocástica. A intensidade $\lambda$ do processo de Poisson determina o valor esperado da medida estocástica $M: \mathbb{E}[M(A)]=\lambda|A|$, sendo que $|A|$ é a medida de Lebesgue de $A$. $M$ que representa a medida estocástica do salto associada ao processo de Poisson $N$. O processo de Poisson pode ser representado em termos da medida estocástica $M$, como segue:

$$
N_{t}(\omega)=M(\omega,[0 ; t])=\int_{[0 ; t]} M(\omega, d s) .
$$

As propriedades do processo de Poissson podem ser traduzidas em propriedades para a medida $M$, i.e., para intervalos desconectados $\left[t_{1} ; t_{1}^{\prime}\right], \ldots,\left[t_{n} ; t_{n}^{\prime}\right]$ :

1. $M\left(\left[t_{k} ; t_{k}^{\prime}\right]\right)$ é um número de saltos de um processo de Poisson em $\left[t_{k} ; t_{k}^{\prime}\right]$ e é um v.e. com distribuição de Poisson com parâmetro $\lambda\left(t_{k}^{\prime}-t_{k}\right)$. 
2. Para dois intervalos desconectados $j \neq k, M\left(\left[t_{j} ; t_{j}^{\prime}\right]\right)$ e $M\left(\left[t_{k} ; t_{k}^{\prime}\right]\right)$ são variáveis estocásticas independentes.

3. De uma maneira geral, para qualquer conjunto mensurável $A, M(A)$ segue uma distribuição de Poisson com parâmetro $\lambda|A|$, sendo que $|A|=\int_{A} d x$ é a medida de Lebesgue de $A$.

A medida estocástica $M$ também pode ser entendida como a derivada do processo de Poisson. Cabe lembrar que, cada trajetória $t \longmapsto N_{t}(\omega)$ do processo de Poisson é uma função que cresce em degraus. Assim, a derivada é uma medida positiva. De fato ela é uma sobreposição da massa associada a medida de Dirac localizada nos instantes de tempo onde ocorrem os saltos:

$$
\frac{d}{d t} N_{t}(\omega)=M(\omega, d t) \text { onde } M=\sum_{i \geq 1} \delta_{T_{i}(\omega)} .
$$

Desta maneira, é possível associar uma medida estocástica a um processo compensado de Poisson $\tilde{N}_{t}$ [Subseção 1.3.4]:

$$
\tilde{M}(\omega, A)=M(\omega, A)-\int_{A} \lambda d t=M(\omega, A)-\lambda|A| .
$$

Assim, a v.e. $\tilde{M}(A)$ possui $\mathbb{E}[\tilde{M}(A)]=0$ e $\mathbb{V}[\tilde{M}(A)]=\lambda|A|$. É importante notar que a medida $\tilde{M}(A)$, ao contrário da medida $M(A)$, não é necessariamente positiva e de valores inteiros (medida de contagem). Ela é uma medida sinalizada (possui partes positivas e negativas). $\tilde{M}$ é um exemplo de medida estocástica compensada e a medida $A \longmapsto \lambda|A|$ é denominado compensador de $M$. O compensador é apenas a intensidade do processo $\lambda$ multiplicada pela medida de Lebesgue $|A|$, que é igual a $\lambda|A|=\mathbb{E}[M(A)]$ e $\tilde{M}$ é a versão centrada de $M$.

Esta construção pode ser generalizada de várias maneiras, o que permite estabelecer os conceitos de medida estocástica de Poisson.

\subsubsection{Medida Estocástica de Poisson ou Processos Pontuais}

A medida $M$ definida em (1.52) especifica uma medida estocástica de contagem em $\mathbb{R}^{+}$, tal que, para qualquer conjunto mensurável $A \subset \mathbb{R}^{+}, \mathbb{E}[M(A)]$ é dado por $\lambda$ multiplicado pela medida de Lebesgue $A$. É possível estender esta construção para 
situações mais genéricas, ao substituir $A \subset \mathbb{R}^{+}$por $E \subset \mathbb{R}^{d}$ e a medida de Lebesgue pela medida de Radon $\mu$ em $E$ [Definição B.2], como segue:

Definição 1.14 Medida Estocástica de Poisson $\operatorname{Seja}(\Omega, \mathcal{F}, \mathbb{P})$ um espaço probabilístico, $E \subset \mathbb{R}^{d}$ e $\mu$ uma medida (positiva) de Radon em $(E, \mathcal{E})$. Uma medida estocástica de Poisson em E, com intensidade $\mu$, é uma medida estocástica de valores inteiros:

$$
\begin{aligned}
M: & \Omega \times \mathcal{E} \longrightarrow \mathbb{N} \\
(\omega, A) & \longmapsto M(\omega, A),
\end{aligned}
$$

tal que:

1. Para $\omega \in \Omega, M(\omega, \cdot)$ é uma medida de Radon em $E$ de valores inteiros, i.e., para qualquer conjunto contornado $A \subset E, M(A)<\infty$ é uma medida estocástica de valores inteiros.

2. Para todo conjunto mensurável $A \subset E, M(\cdot, A)=M(A)$ é uma v.e. de Poisson com parâmetro $\mu(A)$ :

$$
\forall k \in \mathbb{N}, \mathbb{P}(M(A)=k)=\exp \{-\mu(A)\} \frac{\mu(A)^{k}}{k !} .
$$

3. Para conjuntos mensuráveis desconectados $A_{1}, \ldots, A_{n} \in \mathcal{E}$, as variáveis $M\left(A_{1}\right), \ldots, M\left(A_{n}\right)$ são independentes.

Dado uma medida de Radon $\mu$, é possível construir um medida estocástica de Poisson com intensidade $\mu$.

\section{Proposição 1.8 Construção de Medidas Estocásticas de Poisson Para qual-} quer medida de Radon $\mu$ em $E \subset \mathbb{R}^{d}$ existe uma medida estocástica de Poisson $M$ em E com intensidade $\mu$.

Prova $\operatorname{Se} \mu(E)<\infty$ :

1. Seja $X_{1}, X_{2}, \ldots$ variáveis estocásticas i.i.d., tal que $\mathbb{P}\left(X_{i} \in A\right)=\frac{\mu(A)}{\mu(E)}$, para qualquer $A \in \mathcal{E}$. 
2. Seja $M(E)$ uma v.e. de Poisson em $(\Omega, \mathcal{F}, \mathbb{P})$, com esperança $\mu(E)$, independentemente de $X_{i}$.

3. Defina $M(A)=\sum_{i=1}^{M(E)} 1_{A}\left(X_{i}\right)$, para todo $A \in \mathcal{E}$.

É possível verificar que $M$ é uma medida estocástica de Poisson com intensidade $\mu$.

Se $\mu(E)=\infty$, e $\mu$ é uma medida de Radon, é possível representar $E \subset \mathbb{R}^{d}$ como $E=\bigcup_{i=1}^{\infty} E_{i}$, sendo que $\mu\left(E_{i}\right)<\infty$. Desta maneira, pode-se construir uma medida estocástica de Poisson $M_{i}(\cdot)$, de intensidade igual a $\mu\left(E_{i}\right)$. Considere que $M_{1}(\cdot), M_{2}(\cdot), \ldots$ são independentes e defina $M(A)=\sum_{i=1}^{\infty} M_{i}(A)$ para qualquer $A \in \mathcal{E}$. Pelas propriedades de sobreposição (p.35) e afinamento (p.35) de um processo de Poisson, $M$ é uma medida estocástica de Poisson com intensidade $\mu$.

A construção apresentada na Prova acima mostra que de fato qualquer medida estocástica de Poisson em $E$ pode ser interpretada como uma medida de contagem associada a uma seqüência de pontos ${ }^{5} \mathrm{em} E$, i.e., se existir $\left\{X_{n}(\omega), n \geq 1\right\}$, tal que,

$$
\forall A \in \mathcal{E}, \quad M(\omega, A)=\sum_{n \geq 1} 1_{A}\left(X_{n}(\omega)\right) .
$$

Assim, $M$ é a soma de massas de Dirac alocadas nos pontos estocásticos $\left\{X_{n}\right\}_{n \geq 1}$ :

$$
M=\sum_{n \geq 1} \delta_{X_{n}}
$$

Dado que é necessário que $M(A)$ seja finito para qualquer subconjunto compacto $A \subset$ $E$, então, a seqüência $\left\{X_{n}\right\}_{n \geq 1}: A \cap\left\{X_{n}, n \geq 1\right\}$ está restrita a qualquer subconjunto $A \subset E$, não sendo possível $A \subseteq E$.

Uma medida estocástica de Poisson em $E$ pode ser considerada uma v.e. assumindo valores em $\mathcal{M}(E)$, que é a representação do conjunto de medida de Radon em $E$, cuja topologia pode ser definida como: a seqüência $\mu_{n}$ de medidas de Radon em $E \subset \mathbb{R}^{d}$ convergem para uma medida $\mu$ se para qualquer $f: E \longrightarrow \mathbb{R}$, dado um suporte compacto, $\int f d \mu_{n} \stackrel{n \rightarrow \infty}{\longrightarrow} \int f d \mu$. Assim, os conceitos de convergências de variá-

5 É por esta razão que a medida estocástica de Poisson também é denominada processo pontual de Poisson, entrentanto, o termo processo é inapropriado pois a variável tempo não influencia a seqüência de variáveis estocásticas. 
veis estocásticas apresentados na Subseção D também são aplicáveis para as medidas estocásticas de Poisson. [Kallenberg (2002), p.317]:

Proposição 1.9 Convergência de Medidas Estocásticas de Poisson Seja $\left\{M_{n}\right\}_{n \geq 1}$ uma seqüência de medidas estocásticas de Poisson em $E \subset \mathbb{R}^{d}$ com intensidades $\left\{\mu_{n}\right\}_{n \geq 1}$. A seqüência de variáveis estocásticas $\left\{M_{n}\right\}_{n \geq 1}$ convergirá em distribuição se e somente se as intensidades $\left\{\mu_{n}\right\}$ convergirem para uma medida de Radon $\mu$. Desta maneira, $M_{n} \Longrightarrow M$, sendo que $M$ é uma medida estocástica de Poisson com intensidade $\mu$.

\subsubsection{Medida Estocástica Compensada de Poisson}

Da mesma maneira que foi definido o processo compensado de Poisson a partir de um processo de Poisson [Subseção 1.3.4], é possível construir uma medida estocástica compensada de Poisson $\tilde{M}$ ao subtrair de $M$ a intensidade da medida $\mu$ :

$$
\tilde{M}(A)=M(A)-\mu(A) .
$$

A partir da definição de medida estocástica de Poisson [Definição 1.14], pode-se deduzir que para quaisquer conjuntos compactos desconectados $A_{1}, \ldots, A_{n} \in \mathcal{E}$, as variáveis $\tilde{M}\left(A_{1}\right), \ldots, \tilde{M}\left(A_{2}\right)$ são independentes e que

$$
\mathbb{E}\left[\tilde{M}\left(A_{i}\right)\right]=0 \quad \mathbb{V}\left[\tilde{M}\left(A_{i}\right)\right]=\mu\left(A_{i}\right), \text { para } 1 \leq i \leq n .
$$

\subsubsection{Construindo Processos com Saltos}

Considere uma medida estocástica de Poisson $M$ em $E=[0 ; T] \times \mathbb{R}^{d} \backslash\{0\}$. Esta medida pode descrever uma medida de contagem associada a uma configuração estocástica de pontos $\left\{T_{n}, Y_{n}\right\} \in E$ :

$$
M=\sum_{n \geq 1} \delta_{\left\{T_{n}, Y_{n}\right\}}
$$

Intuitivamente, cada ponto $\left\{T_{n}(\omega), Y_{n}(\omega)\right\} \in[0 ; T] \times \mathbb{R}^{d}$ corresponde a uma observação realizada no instante de tempo $T_{n}$ que é descrita por uma v.e. (diferente de zero) $Y_{n}(\omega) \in \mathbb{R}^{d}$. Assim, como é desejável que a primeira coordenada $t$ seja interpretada 
como a variável tempo, será introduzida uma filtragem $\mathcal{F}_{t}$ em $(\Omega, \mathcal{F}, \mathbb{P})$ [Definição 1.2]. A medida estocástica de Poisson será adaptada em relação à $\mathcal{F}_{t}$ (não-antecipável) se:

1. $\left\{T_{n}\right\}_{n \geq 1}$ são tempos estocásticos não-antecipáveis.

2. $Y_{n}$ é revelada em $T_{n}: T_{n}$ é $\mathcal{F}_{T_{n}}$-mensurável.

Para cada $\omega, M(\omega, \cdot)$ é uma medida em $E=[0 ; T] \times \mathbb{R}^{d} \backslash\{0\}$, sendo possível definir integrais em relação a esta medida, de maneira análoga a Subseção B.2. Inicialmente, para funções simples $f=\sum_{i=1}^{n} c_{i} 1_{A_{i}}$, sendo que $c_{i}>0$ e $A_{i} \subset E$ são conjuntos mensuráveis desconectados. $M(f)=\sum_{i=1}^{n} c_{i} M\left(A_{i}\right)$ será definida como uma v.e. com esperança $\mathbb{E}[M(f)]=\sum_{i=1}^{n} c_{i} \mu\left(A_{i}\right)$. A seguir, para uma função mensurável positiva $f: E \longrightarrow\left[0 ; \infty\left[\right.\right.$, defina $M(f)=\lim _{n \rightarrow \infty} M\left(f_{n}\right)$, sendo que $f_{n} \uparrow f$ é uma seqüência crescente de funções simples. Pelo teorema da convergência monotônica [Kallenberg (2002), Teorema 1.19, p.11], $M(f)$ é uma v.e. que assume valores em $[0 ; \infty[\cup\{\infty\}$ e possui esperança igual a $\mathbb{E}[M(f)]=\mu(f)$. Para uma função mensurável de valores reais $f: E \longrightarrow \mathbb{R}$, é possível decompor $f$ nas suas partes positivas e negativas, i.e., $f=f_{+}-f_{-}$. Se $f$ satisfizer

$$
\mu(|f|)=\int_{[0 ; T]} \int_{\mathbb{R}^{d} \backslash\{0\}}|f(s, y)| \mu(d s \times d y)<\infty,
$$

então, as variáveis estocásticas positivas $M\left(f_{+}\right)$e $M\left(f_{-}\right)$têm esperanças finitas, i.e., $\mathbb{E}\left[M\left(f_{+}\right)\right]=\mu\left(f_{+}\right) \leq \mu(|f|)<\infty$. Em particular, $M\left(f_{+}\right)$e $M\left(f_{-}\right)$são q.c. finitas. Assim é possível definir $M(f):=M\left(f_{+}\right)-M\left(f_{-}\right)$, sendo que $M(f)$ é definida como uma v.e. com esperança

$$
\mathbb{E}[M(f)]=\mu(f)=\int_{[0 ; T]} \int_{\mathbb{R}^{d} \backslash\{0\}} f(s, y) \mu(d s \times d y) .
$$

Integrar $f$ em relação $M$ até o instante $t$, ou seja, ao restringir a integração ao suporte $[0 ; t] \times \mathbb{R}^{d} \backslash\{0\}$ garante a não-antecipalidade do processo estocástico:

$$
\begin{aligned}
X_{t} & =\int_{[0 ; t]} \int_{\mathbb{R}^{d} \backslash\{0\}} f(s, y) M(d s \times d y) \\
& =\sum_{\left\{n, T_{n} \in[0 ; t]\right\}} f\left(T_{n}, Y_{n}\right) .
\end{aligned}
$$

A somatória é efetuada sobre os eventos $\left\{T_{n}, Y_{n}\right\}$ que ocorrem antes de $t$, i.e., $T_{n} \leq$ t. $\left\{X_{t}(f)\right\}_{t \in[0 ; T]}$ é, então, um processo com saltos, os quais ocorrem nos intantes de 
tempo $T_{n}$, com aplitudes dada por $f\left(T_{n}, Y_{n}\right)$. Cabe lembrar, que esta construção faz sentido apenas quando $f$ atende a restrição dada pela equação (1.61).

De maneira análoga, sob a condição (1.61), é possível definir uma integral de $f$ com relação ao medida estocástica compensada de Poisson $\tilde{M}$. O processo resultante, denominado integral compensada, é uma martingale:

\section{Proposição 1.10 Integral Compensada de Poisson Seja M uma medida} estocástica de Poisson não-antecipável em $E=[0 ; T] \times \mathbb{R}^{d} \backslash\{0\}$ com intensidade $\mu$, sendo sua respectiva medida estocástica compensada $\tilde{M}=M-\mu$, e $f: E \longrightarrow \mathbb{R}^{d}$ atendendo a condição (1.61). Assim, este processo

$$
\begin{aligned}
X_{t} & =\int_{0}^{t} \int_{\mathbb{R}^{d} \backslash\{0\}} f(s, y) \tilde{M}(d s d y) \\
& =\int_{0}^{t} \int_{\mathbb{R}^{d} \backslash\{0\}} f(s, y) M(d s d y)-\int_{0}^{t} \int_{\mathbb{R}^{d} \backslash\{0\}} f(s, y) \mu(d s d y)
\end{aligned}
$$

é uma martingale.

Cabe lembrar que, apesar das integrais definidas nesta seção serem estocásticas, elas são determinísticas para cada trajetória, i.e., para qualquer $\omega \in \Omega$.

\subsubsection{Processo Pontual Registrado}

Como foi observado anteriormente, a medida estocástica de Poisson em $[0 ; T] \times$ $\mathbb{R}^{d}$ pode ser representada como uma medida de contagem:

$$
M(\omega, \cdot)=\sum_{n \geq 1} \delta_{\left\{T_{n}(\omega), Y_{n}(\omega)\right\}}
$$

para uma seqüência estocástica de pontos $\left\{T_{n}, Y_{n}\right\}_{n \geq 1}$ em $[0 ; T] \times \mathbb{R}^{d}$. Esta representação permite definir medidas estocásticas de valores inteiros com propriedades de dependência. Dado uma seqüência estocástica $\left\{T_{n}, Y_{n}\right\} \in[0 ; T] \times E$, sendo que $\left\{T_{n}\right\}_{n \geq 1}$ é uma seqüência de tempos estocásticos não-antecipáveis que descreve a ocorrência de certos eventos e $Y_{n} \in E \subseteq \mathbb{R}^{d}$ a quantidade observada no instante de tempo $T_{n}$ ( $Y_{n}$ é $\mathcal{F}_{T_{n}}$-mensurável), é possível definir uma medida de contagem $M$ pela equação (1.65). $M$ representa uma medida estocástica de valores inteiros em $[0 ; T] \times E$ e a seqüência $\left\{T_{n}, Y_{n}\right\} \in[0 ; T] \times E$ é denominado processo pontual registrado de contagem. As variáveis estocásticas $\left\{Y_{n}\right\}_{n \geq 1}$ são denominadas registros. 


\section{Definição 1.15 Processo Pontual Registrado Um processo pontual} registrado em $\left(\Omega, \mathcal{F},\left(\mathcal{F}_{t}\right), \mathbb{P}\right)$ é uma seqüência $\left\{T_{n}, Y_{n}\right\}_{n \geq 1}$, sendo que

1. $\left\{T_{n}\right\}_{n \geq 1}$ é uma seqüência crescente de tempos estocásticos não-antecipáveis com $T_{n} \longrightarrow \infty$-q.c. a medida que $n \longrightarrow \infty$.

2. $\left\{Y_{n}\right\}_{n \geq 1}$ é uma seqüência de variáveis estocásticas que assume valores em E.

3. O valor de $Y_{n}$ é revelado no instante $T_{n}: Y_{n}$ é $\mathcal{F}_{T_{n}}$-mensurável.

A condição $T_{n} \longrightarrow \infty$ garante que o número de eventos que ocorrem em $[0 ; T]$ é finito-q.c. Para cada $\omega \in \Omega, M(\omega, \cdot)$ é uma medida de contagem em $[0 ; T] \times E$. Se $\mu$ for uma medida difusa (p.197), i.e., $\mu(\{t, y\})=0$ para todos os pontos $\{t, y\} \in$ $[0 ; T] \times E$, então, com probabilidade 1 , cada ponto ocorre no máximo uma única vez: $M(\{t, y\})=0$ ou 1 .

O processo pontual registrado não possui a propriedade de indepedência da medida estocástica de Poisson, i.e., se $A_{1} \cap A_{2}=\varnothing$, então, $M\left([0 ; t] \times A_{1}\right)$ e $M\left([0 ; t] \times A_{2}\right)$ não são independentes, nem são variáveis estocásticas de Poisson. Este processo permite distribuições arbitrárias e uma estrutura de dependência. Qualquer medida estocástica de Poisson em $[0 ; T] \times \mathbb{R}$ pode ser representada pela equação (1.65), mas, $\left\{T_{n}\right\}$ não necessariamente converge para infinito $T_{n} \longrightarrow \infty$, o que significa que nem todos as medidas estocásticas de Poisson podem ser representadas por processos pontuais registrados. Apenas as medidas estocásticas de Poisson que atendem a condição $\mu\left([0 ; T] \times \mathbb{R}^{d}\right)<\infty$ podem ser representadas por processos pontuais registrados.

Para a função $f:[0 ; T] \times E \quad \longrightarrow \quad \mathbb{R}^{d}$ que atende a condição $\int_{[0 ; T] \times E}|f(t, y)| \mu(d t d y)<\infty$, é possível construir uma integral em relação à medida $M$ :

$$
M(f)=\int_{[0 ; T] \times E} f(t, y) M(d t d y)=\sum_{n \geq 1} f\left(T_{n}, Y_{n}\right) .
$$

Também é possível construir um processo com saltos a partir de $f$, de maneira análoga à Subseção 1.4.1, i.e.,

$$
X_{t}=\int_{[0 ; t] \times \mathbb{R}^{d} \backslash\{0\}} f(s, y) M(d s d y)=\sum_{\left\{n, T_{n} \in[0 ; t]\right\}} f\left(T_{n}, Y_{n}\right) .
$$


$\left\{X_{t}(f)\right\}_{t \in[0 ; T]}$ é um processo não-antecipável com saltos, sendo que suas trajetórias cadlag possuem saltos que são orientadas por um processo pontual registrado $M$, ou seja, os saltos ocorrem nos instantes $\left\{T_{n}\right\}_{n \geq 1}$ e suas amplitudes são dadas por $f\left(T_{n}, Y_{n}\right)$. Esta construção permite gerar processos com saltos a partir de processos pontuais registrados de maneira sistemática.

Por outro lado, para cada processo cadlag $X=\left\{X_{t}\right\}_{t \in[0 ; T]}$ em $\mathbb{R}^{d}$ é possível associar uma medida estocástica $J_{X}$ em $[0 ; T] \times \mathbb{R}^{d}$ denominada medida de salto. O processo $X$ tem no máximo uma quantidade enumerável de saltos, i.e., $\left\{t \in[0 ; T], \Delta X_{t}=X_{t}-X_{t-} \neq 0\right\}$ é enumerável, sendo que os seus elementos podem ser organizados na seqüência $\left\{T_{n}\right\}_{n \geq 1}$ (não necessariamente crescente), que são os tempos estocásticos dos saltos de $X$. No instante $T_{n}$, o processo $X$ tem uma descontinuidade de tamanho igual a $Y_{n}=X_{T_{n}}-X_{T_{n}-} \in \mathbb{R}^{d} \backslash\{0\} .\left\{T_{n}, Y_{n}\right\}_{n \geq 1}$ define um processo pontual registrado em $[0 ; T] \times \mathbb{R}^{d} \backslash\{0\}$ que contém toda a informação sobre os saltos do processo $X$, i.e., os tempos que ocorrem os saltos $T_{n}$ e o tamanho do salto $Y_{n}$. A medida estocástica associada ao processo $X$, que é representada pela medida $J_{X}$, é denominada medida de salto do processo $X$ :

$$
J_{X}(\omega, \cdot)=\sum_{n \geq 1} \delta_{\left\{T_{n}(\omega), Y_{n}(\omega)\right\}}=\sum_{t \in[0 ; T]}^{\Delta X_{t} \neq 0} \delta_{\left\{t, \Delta X_{t}\right\}} .
$$

De maneira intuitiva, para qualquer conjunto mensurável $A \subset \mathbb{R}^{d}, J_{X}([0 ; t] \times A)$ é a quantidade de saltos do processo $X$ que ocorrem entre 0 e $t$, cujo tamanho (amplitude) pertence ao conjunto $A$. A medida estocástica $J_{X}$ contém toda a informação sobre as descontinuidades (saltos) do processo $X$, i.e., ela diz quando os saltos ocorrem e quão grande será. Apesar disto, $J_{X}$ não diz nada a respeito da componente contínua do processo $X$. É possível perceber que as trajetórias observadas do processo $X$ possuem continuidades se e somente se $J_{X}=0$-q.c. (não ocorrência de saltos).

Todas as quantidades que envolvem os saltos de $X$ podem ser computadas ao integrar várias funções em relação a $J_{X}$, e.g., se $f(t, y)=y^{2}$, então, é possível obter a soma quadrática dos saltos de $X$, pela expressão

$$
\int_{[0 ; T] \times R} y^{2} J_{X}(d t d y)=\sum_{t \in[0 ; T]}\left(\Delta X_{t}\right)^{2} .
$$

Seguem outros exemplos interessantes: 
Exemplo 1.1 - Medida de Salto de um Processo de Poisson A medida de salto de um processo de Poisson [Definição (1.13)] é dada por $J_{N}=\sum_{n \geq 1} \delta_{\left\{T_{n}, 1\right\}}$ :

$$
J_{N}([0 ; t] \times A)=\left\{\begin{array}{ll}
\#\left\{i \geq 1, T_{i} \in[0 ; t]\right\} & \text { se } 1 \in A \\
=0 & \text { se } 1 \notin A
\end{array} .\right.
$$

Exemplo 1.2 Considere uma seqüência não-antecipável de tempos estocásticos $T_{1}, T_{2}, \ldots$ e $\left\{X_{n}\right\}_{n \geq 1}$ uma seqüência de variáveis estocásticas, tal que, $X_{n}$ é revelada no instante $T_{n}$. Assim,

$$
X_{t}=\sum_{n \geq 1} X_{n} 1_{\left[T_{n} ; T_{n+1}\right.}
$$

define um processo cadlag com tempo de ocorrência dos saltos $T_{n}$ e tamanho $\Delta X_{T_{n}}=$ $X_{n}-X_{n-1}$. A medida de salto é dado por

$$
J_{X}=\sum_{n \geq 1} \delta_{\left\{T_{n}, X_{n}-X_{n-1}\right\}}
$$

Se $T_{n} \longrightarrow \infty$-q.c., então, $\left\{T_{n}, X_{n}-X_{n-1}\right\}$ define um processo pontual registrado.

Cabe notar que, se $X_{t}$ é um processo contruído a partir de uma medida estocástica de Poisson $M$, dada pela equação (1.67), i.e.,

$$
M=\sum_{n \geq 1} \delta_{\left\{T_{n}, Y_{n}\right\}} \quad X_{t}=\int_{[0 ; t] \times \mathbb{R}^{d} \backslash\{0\}} f(s, y) M(d s d y),
$$

então, a medida de salto $J_{X}$ pode ser expressa em termos da medida estocástica de Poisson $M$ :

$$
J_{X}=\sum_{n \geq 1} \delta_{\left\{T_{n}, f\left(T_{n}, Y_{n}\right)\right\}}
$$




\section{Capítulo 2 Processos Estocásticos de Lévy}

Os passeios aleatórios, que podem ser entendidos como a soma finita de variáveis aleatórias, são processos estocásticos em tempo discreto. Por outro lado, os processos com incrementos independentes e estacionários em tempo-contínuo são denominados processos de Lévy. Estes processos são os alicerces para construir modelos em tempocontínuo em finanças. Os processos de Poisson e Wiener são casos particulares dos processos de Lévy. Estes dois processos são de grande interesse, pois é possível entender os processos de Lévy, de uma maneira generalizada, como a superposição de um processo de Wiener e um número (possivelmente infinito) de processos de Poisson independentes. Assim, podemos definir um processo de Lévy da seguinte maneira:

Definição 2.1 Processo de Lévy Um processo estocástico cadlag $X=\left\{X_{t}\right\}_{t \geq 0}$ em $(\Omega, \mathcal{F}, \mathbb{P})$ com valores em $\mathbb{R}^{d}$ tal que $X_{0}=0$ é um processo de Lévy se e somente se possuir as seguintes propriedades:

1. Incrementos independentes: para qualquer seqüência de tempos $t_{0} \cdots t_{n}$, as variáveis estocásticas $X_{t_{0}}, X_{t_{1}}-X_{t_{0}}, \ldots, X_{t_{n}}-X_{t_{n-1}}$ são independentes.

2. Incrementos estacionários: a distribuição de $X_{t+h}-X_{t}$ não depende de $t$.

3. Continuidade estocástica: $\forall \varepsilon>0, \lim _{h \rightarrow 0} \mathbb{P}\left(\left|X_{t+h}-X_{t}\right| \geq \varepsilon\right)=0$.

A terceira condição não implica que os caminhos observados sejam contínuos, como já foi observado na propriedade 4 da Proposição 1.6 de um processo de Poisson. Isto serve para excluir processos com saltos em tempos determinísticos, o que pode ser entendido como efeitos já programados, que não são de interesse para a modelagem de eventos estocásticos. Isto quer dizer que dado um instante de tempo $t$, a probabilidade de se observar um salto em $t$ é igual a zero, ou seja, as descontinuidades ocorrem em tempos estocásticos.

Se for observado um processo de Lévy em intervalos de tempo regulares $0, \Delta, 2 \Delta, \ldots$ é possível obter um passeio aleatório ao definir $S_{n}(\Delta) \triangleq X_{n \Delta}$, sendo 
que $S_{n}(\Delta)=\sum_{k=0}^{n-1} Y_{k}$ e $Y_{k}=X_{(k+1) \Delta}-X_{k \Delta}$ são variáveis estocásticas i.i.d. com distribição igual a $X_{\Delta}$. Como isto pode ser efetuado para qualquer intervalo observado $\Delta$ o que se percebe é que ao se especificar um processo de Lévy automaticamente também se está especificando uma família inteira de passeios aleatórios $S_{n}(\Delta)$, i.e., estes processos correspondem a observações dos processo de Lévy $X$ em freqüências diferentes.

Ao escolher $n \Delta=t$ tem se que para qualquer $t>0$ e $n \geq 1, X_{t}=S_{n}(\Delta)$ pode ser representado como a soma de variáveis estocásticas i.i.d. com distribuição igual a de $X_{t / n}$. Assim, $X_{t}$ pode ser divido em $n$ partes i.i.d. Um distribuição que apresenta esta propriedade é denominada infinitamente divisível.

\section{Definição 2.2 Divisibilidade Infinita Uma distribuição de probabilidade $F$} em $\mathbb{R}^{d}$ é denominada infinitamente divisivel se e somente se para qualquer $n \geq 2$, existem $n$ variáveis estocásticas i.i.d. $Y_{1}, \cdots, Y_{n}$ tal que $Y_{1}+\cdots+Y_{n}$ tem distribuição $F$.

Dado que a distribuição da soma de variáveis estocásticas i.i.d. é dado pela convolução da distribuição individual de cada v.e., sendo que $\mu$ representa a distribuição de cada $Y_{k}$, então, $F=\mu^{* n}$ é a convolução de ordem $n$ de $\mu$. Assim, uma distribuição infinitamente divisível pode ser definida como a distribuição $F$, sendo que a raíz $n$-ésima desta distribuição é uma distribuição de probabilidade, para qualquer $n \geq 2$.

Desta maneira, se $X$ é um processo de Lévy, para qualquer $t>0$ a distribuição de $X_{t}$ é infinitamente divisível. Esta propriedade estabelece um restrição às possíveis escolhas de distribuições para $X_{t}$, o que não ocorre com os incrementos de um passeio aleatório em tempo-discreto que podem assumir arbitrariamente qualquer distribuição. Os exemplos mais comuns de distribuições infinitamente divisíveis são a distribição gaussiana, a distribuição gama, distribuições $\alpha$-estáveis e a distribuição de Poisson. Uma v.e. que assume qualquer uma destas distribuições pode ser decomposta na soma de $n$ partes (variáveis estocásticas) i.i.d. que assumem a mesma distribuição com parâmetros modificados. Por exemplo, se $X \stackrel{d}{=} \mathcal{N}\left(\mu, \sigma^{2}\right)$, então, é possível decompor $X$ em $X=\sum_{k=0}^{n-1} Y_{k}$, sendo que $Y_{k}$ são i.i.d. com distribuição $\mathcal{N}\left(\mu / n, \sigma^{2} / n\right)$. Outros exemplos, menos triviais, são as distribuições log-gaussiana, Pareto, t de Student. 
Um exemplo de distribuição que não é infinitamente divisível é a distribuição uniforme definida em um intervalo.

De maneira análoga, dado uma distribuição infinitamente divisível $F$, é possível perceber que, para qualquer $n \geq 1$, ao segregar a distribuição em $n$ componentes i.i.d., obtém-se um passeio aleatório de passos de tamanho igual a $1 / n$, tal que a distribuição no instante $t=1$, é dado pela distribuição $F$. No limite, este procedimento pode ser utilizado para construir processos contínuos de Lévy $\left\{X_{t}\right\}_{t \geq 0}$ tal que a distribuição de $X_{1}$ é dado pela seguinte proposição:

Proposição 2.1 Processo de Lévy infinitamente divisível Seja $\left\{X_{t}\right\}_{t \geq 0}$ um processo de Lévy. Então, para qualquer $t, X_{t}$ possui uma distribuição infinitamente divisível. De maneira contrária, se F é uma distribuição infinitamente divisível, então, existe um processo $X_{t}$, tal que a distribuição de $X_{1}$ é dado por $F$.

Defina a seguinte função característica de $X_{t}$ :

$$
\Phi_{t}(z) \triangleq \Phi_{X_{t}}(z) \triangleq \mathbb{E}\left[\exp \left(i z X_{t}\right)\right], \quad z \in \mathbb{R}^{d} .
$$

Para $t>s$, e representado $X_{t+s}=X_{s}+\left(X_{t+s}-X_{s}\right)$ e utilizando o fato de que $X_{t+s}-X_{s}$ é independente de $X_{s}$ (independência dos incrementos), obtém-se que $t \longmapsto$ $\Phi_{t}(z)$ é uma função multiplicativa:

$$
\Phi_{t+s}(z) \triangleq \Phi_{X_{t+s}}(z)=\Phi_{X_{s}} \Phi_{X_{t+s}-X_{s}} .
$$

Dado a estacionariedade dos incrementos, i.e., $\Phi_{X_{t+s}-X_{s}}$ não depende de $s$, tem-se que

$$
\Phi_{t+s}(z)=\Phi_{X_{s}} \Phi_{X_{t+s}-X_{s}}=\Phi_{X_{s}} \Phi_{X_{t}} .
$$

A continuidade estocástica permite que a função $t \longmapsto \Phi_{t}(z)$ convirja em distribuição $X_{t} \stackrel{d}{\longrightarrow} X_{s}$ quando $t \longrightarrow s$. Assim, pela Proposição D.2, $\Phi_{X_{t}} \longrightarrow \Phi_{X_{s}}$ quando $t \longrightarrow s$, e que $t \longmapsto \Phi_{t}(z)$ é uma função contínua em $t$. Em conjunto com a propriedade de função multiplicativa $\Phi_{t+s}(z)=\Phi_{X_{s}} \Phi_{X_{t}}$ tem-se que $t \longmapsto \Phi_{t}(z)$ é uma função exponencial:

Proposição 2.2 Função característica de um processo de Lévy $S e X=\left\{X_{t}\right\}_{t \geq 0}$ um processo de Lévy em $\mathbb{R}^{d}$, então, existe uma função contínua $\psi: \mathbb{R}^{d} \longmapsto \mathbb{R}$ denomi- 
nada expoente característico de $X$, tal que:

$$
\mathbb{E}\left[\exp \left(i z \cdot X_{t}\right)\right]=\exp \{t \psi(z)\}, \quad z \in \mathbb{R}^{d} .
$$

Cabe lembrar que, pela equação (C.15), que define uma função geratriz de cumulantes, é possível perceber que $\psi$ é a função geratriz de cumulantes de $X_{1}$, sendo que $\psi=\Psi_{X_{1}}$ e que a função geratriz de cumulantes de $X_{t}$ varia linearmente em $t$, i.e., $\Psi_{X_{t}}=t \Psi_{X_{1}}=t \psi$. Portanto, a distribuição de $X_{t}$ é determinada pelo conhecimento da distribuição de $X_{1}$. Isto significa que o único grau de liberdade, ao se especificar um processo de Lévy, é determinar a distribuição de $X_{t}$ para um período unitário $t=1$.

\subsection{Processo Composto de Poisson}

Um processo composto de Poisson pode ser definido como:

Definição 2.3 Processo composto de Poisson Um processo composto de Poisson com intensidade $\lambda>0$ e tamanho do salto com distribuição $f$ é um processo estocástico $X_{t}$ definido como:

$$
X_{t}=\sum_{i=1}^{N_{t}} Y_{i},
$$

onde os tamanhos dos saltos $Y_{i}$ são i.i.d. com distribuição $f$ e $\left\{N_{t}\right\}$ é um processo de Poisson com intensidade $\lambda$, independente de $\left\{Y_{i}\right\}_{i \geq 0}$.

Um processo composto de Poisson apresenta as seguintes propriedades:

1. As trajetórias observadas de $X$ são funções cadlag constantes por partes.

2. Os tempos de ocorrência dos saltos $\left\{T_{i}\right\}_{i \geq 0}$ apresentam a mesma distribuição dos tempos de ocorrência de um processo de Poisson $N_{t}$, i.e., eles podem ser expressos como a soma parcial de variáveis estocásticas exponenciais independentes de parâmetro $\lambda$.

3. O tamanho do saltos $\left\{Y_{i}\right\}_{i>0}$ são independentes e identicamente distribuídos com função $f$. 


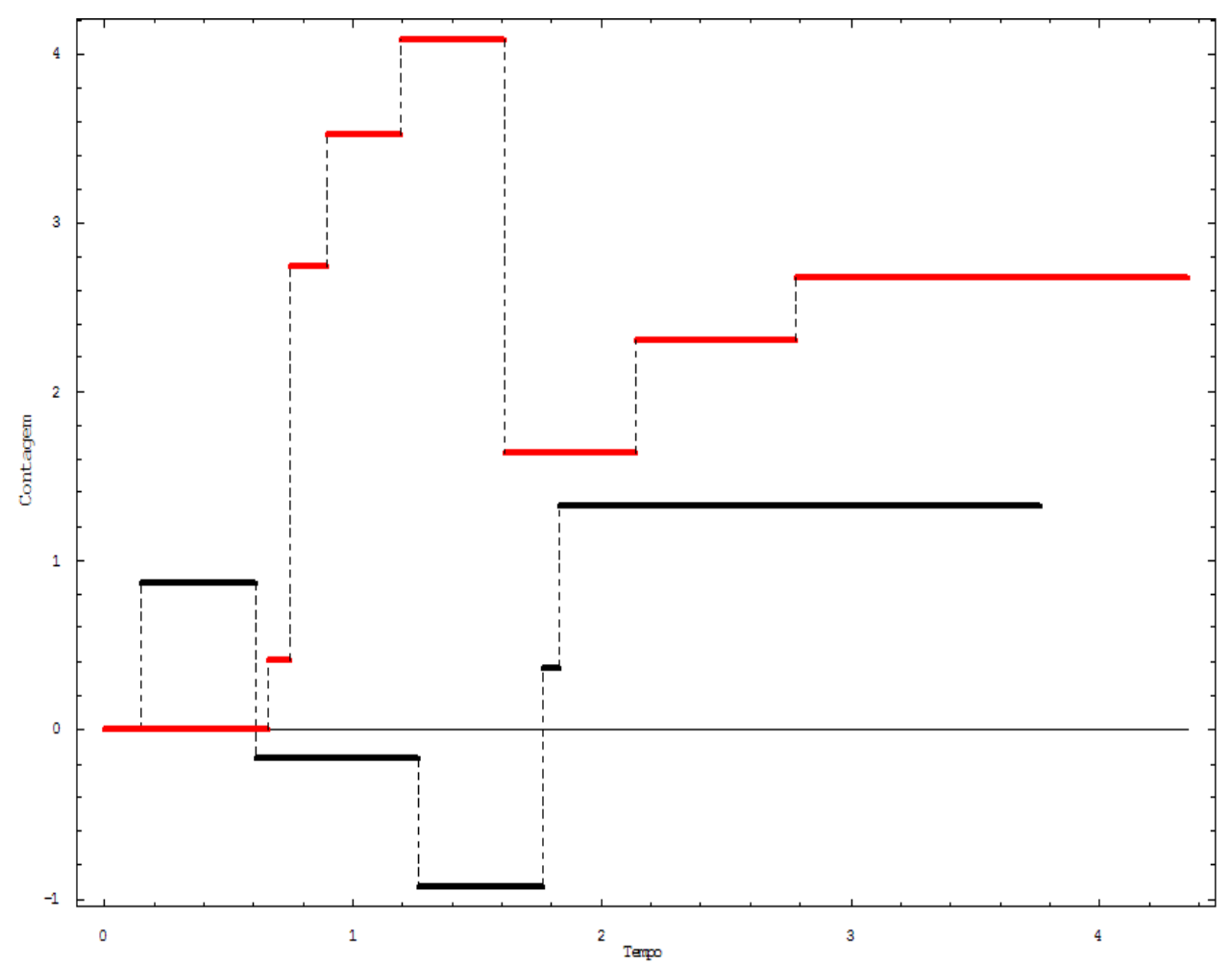

Figura 2.1. Duas trajetórias de um processo composto de Poisson, com tamanho dos saltos distribuídos por $\mathcal{N}(0 ; 1)$.

Um processo de Poisson pode ser expresso como um processo composto de Poisson em $\mathbb{R}$ tal que $Y_{i}=1$. Isto explica a origem do processo composto de Poisson na definição acima.

Seja $R(n), n \geq 0$ um passeio aleatório com incrementos distribuídos por $f$ : $R(n)=\sum_{i=0}^{n} Y_{i}$. Um processo compensado de Poisson $X_{t}$ pode ser obtido ao mudar o tempo determinístico de $R$ por um processo de Poisson $N_{t}: X_{t}=R\left(N_{t}\right)$. Assim, $X_{t}$ descreve a posição do passeio aleatório depois de uma quantidade de estocástica de incrementos ao longo do tempo $N_{t}$.

A Figura (2.1) ilustra uma trajetória típica de um processo composto de Poisson. Cabe ressaltar que a trajetória deste processo é constante por partes. Processos compostos de Poisson são processos de Lévy e, eles são os únicos processos de Lévy que possuem trajetórias observadas constantes por partes, como é apresentado na seguinte proposição: 
Proposição 2.3 $\left\{X_{t}\right\}_{t \geq 0}$ é um processo composto de Poisson se e somente se for um processo de Lévy e suas trajetórias observadas forem funções constantes por partes.

Prova (parte "se") Seja $X=\left\{X_{t}\right\}_{t \geq 0}$ um processo de Lévy com trajetórias constantes por partes. Para mostrar que este processo é um processo composto de Poisson é necessário provar as propriedades 2 e 3 da Definição 2.3. Em relação a propriedade 2, é possível construir, trajetória por trajetória, um processo $N=\left\{N_{t}\right\}_{t \geq 0}$ que enumera a quantidade de saltos de $X$ :

$$
N_{t}=\#\left\{0<s \leq t: X_{s-} \neq X_{s}\right\} .
$$

Como as trajetórias de $X$ são constantes por partes, $X$ tem um quantidade finita de saltos em qualquer intervalo finito, que garante que $N_{t}$ é finito para todo $t$. Desta maneira, $N$ é um processo de contagem. Seja $h<t$, então,

$$
N_{t}-N_{h}=\#\left\{h<s \leq t: X_{s-}-X_{h} \neq X_{s}-X_{h}\right\} .
$$

Logo, $N_{t}-N_{h}$ depende apenas de $\left\{X_{s}-X_{h},\right\}$ para $h \leq s<t$. Pela independência e estacionariedade dos incrementos de $X$ tem-se que $N$ também é independente e estacionário por incrementos. Pelo Lema 1.1 o processo de contagem $N$ é um processo de Poisson, garantindo a Propriedade 2 da Definição 2.3. A partir do processo $N$ é possível calcular o tamanho dos saltos de $X: Y_{s}=X_{S_{n}}-X_{S_{n}-}$, sendo que $S_{n}=$ inf $\left\{t: N_{t} \geq n\right\}$. Para verificar a Propriedade 3 da Definição 2.3 é necessário provar que os tamanhos dos saltos são i.i.d. Inicialmente, é possível verificar que os incrementos de $X$ são independentes condicionados à trajetória de $N$. Para $t>s$, considere os seguintes quatros conjuntos:

$$
\begin{aligned}
A_{1} \in \sigma\left(X_{s}\right) & B_{1} \in \sigma\left(N_{r}, r \leq s\right) \\
A_{2} \in \sigma\left(X_{t}-X_{s}\right) & B_{2} \in \sigma\left(N_{r}-N_{s}, r>s\right),
\end{aligned}
$$

tal que $\mathbb{P}\left(B_{1}\right)>0$ e $\mathbb{P}\left(B_{2}\right)>0$. A independência dos incrementos de $X$ implica em $\left\{X_{r}-X_{s}, r>s\right\}$ e $\left\{X_{r} r \leq s\right\}$ são idependentes. Assim,

$$
\mathbb{P}\left[A_{1} \cap B_{1} \cap A_{2} \cap B_{2}\right]=\mathbb{P}\left[A_{1} \cap B_{1}\right] \mathbb{P}\left[A_{2} \cap B_{2}\right] .
$$

Este resultado permite que: 
1. $A_{1}$ e $B_{1}$ são independentes de $B_{2}$.

2. $A_{2}$ e $B_{2}$ são independentes de $B_{1}$.

3. $B_{1}$ e $B_{2}$ são independentes.

Como conseqüência, é possível expressar a probabilidade condicionada da seguinte maneira:

$$
\begin{aligned}
\mathbb{P}\left[A_{1} \cap A_{2} \mid B_{1} \cap B_{2}\right] & =\frac{\mathbb{P}\left[A_{1} \cap B_{1}\right] \mathbb{P}\left[A_{2} \cap B_{2}\right]}{\mathbb{P}\left[B_{1}\right] \mathbb{P}\left[B_{2}\right]} \\
& =\frac{\mathbb{P}\left[A_{1} \cap B_{1} \cap B_{2}\right] \mathbb{P}\left[A_{2} \cap B_{1} \cap B_{2}\right]}{\mathbb{P}^{2}\left[B_{1}\right] \mathbb{P}^{2}\left[B_{2}\right]} \\
& =\mathbb{P}\left[A_{1} \mid B_{1} \cap B_{2}\right] \mathbb{P}\left[A_{2} \mid B_{1} \cap B_{2}\right]
\end{aligned}
$$

Isto prova que $X_{t}-X_{s}$ e $X_{s}$ são independentes quando condicionados à trajetória de $N$. Em particular, ao escolher $B_{1}=\left\{N_{s}=1\right\}$ e $B_{2}=\left\{N_{t}-N_{s}=1\right\}$ obtém-se que $Y_{1}$ e $Y_{2}$ são condicionalmente independentes. Poderia ter sido escolhido qualquer número de incrementos para $X$ e não apenas dois, o que prova que os incrementos $\left\{Y_{i}\right\}_{i \geq 1}$ são condicionalmente independentes, para qualquer quantidade de incrementos no processo $X$.

Finalmente, para provar que o tamanho dos saltos possuem a mesma distribuição, observe que o processo bi-dimensional $\left\{X_{t}, N_{t}\right\}$ possui incrementos estacionários, logo, para qualquer $n \geq 0$ e para todo $s>h>0$,

$$
\begin{aligned}
\mathbb{E}\left[f\left(X_{h}\right) \mid N_{h}\right. & \left.=1, N_{s}-N_{h}=n\right] \\
& =\mathbb{E}\left[f\left(X_{s+h}-X_{s}\right) \mid N_{s+h}-N_{s}=1, N_{s}-N_{h}=n\right],
\end{aligned}
$$

sendo que $f$ é qualquer função Borel limitada. Isto garante que para qualquer $n \geq 0$, $Y_{1}$ e $Y_{n+2}$ possuem a mesma distribuição.

Prova (parte "somente se") Seja $\left\{X_{t}\right\}_{t \geq 0}$ um processo composto de Poisson.

1. Independência dos incrementos: Seja $0<r<s$ e seja $f$ e $g$ funções limitadas de Borel em $\mathbb{R}^{d}$. Para simplificar a notação, será provado apenas que $X_{r}$ e $X_{s}-X_{r}$ são independentes, apesar do mesmo raciocínio ser aplicado a um número finito de 
incrementos. É necessário mostrar que:

$$
\mathbb{E}\left[f\left(X_{r}\right) g\left(X_{s}-X_{r}\right)\right]=\mathbb{E}\left[f\left(X_{r}\right)\right] \mathbb{E}\left[g\left(X_{s}-X_{r}\right)\right] .
$$

Pela representação $X_{r}=\sum_{i=1}^{N_{r}} Y_{i}$ e $X_{s}-X_{r}=\sum_{i=N_{r}+1}^{N_{s}} Y_{i}$ as seguintes observações podem ser feitas:

- Condicionada a trajetória de $N_{t}$ para qualquer $t \in[0, s], X_{r}$ e $X_{s}-X_{r}$ são independentes porque a primeira expressão depende apenas de $Y_{i} i \leq N_{r}$ e a segunda expressão depende apenas de $Y_{i} i>N_{r}$.

- A esperança de $\mathbb{E}\left[f\left(X_{r}\right) \mid N_{t}, t \leq s\right]$ depende apenas de $N_{r}$ e a esperança de $\mathbb{E}\left[g\left(X_{s}-X_{r}\right)\right]$ depende apenas de $N_{s}-N_{r}$.

Ao considerar a independência dos incrementos do processo de Poisson, é possível escrever:

$$
\begin{aligned}
\mathbb{E}\left[f\left(X_{r}\right) g\left(X_{s}-X_{r}\right)\right] & =\mathbb{E}\left[\mathbb{E}\left[f\left(X_{r}\right) g\left(X_{s}-X_{r}\right) \mid N_{t}, t \leq s\right]\right] \\
& =\mathbb{E}\left[\mathbb{E}\left[f\left(X_{r}\right) \mid N_{t}, t \leq s\right] \mathbb{E}\left[g\left(X_{s}-X_{r}\right) \mid N_{t}, t \leq s\right]\right] \\
& =\mathbb{E}\left[\mathbb{E}\left[f\left(X_{r}\right) \mid N_{r}\right] \mathbb{E}\left[g\left(X_{s}-X_{r}\right) \mid N_{s-r}\right]\right] \\
& =\mathbb{E}\left[\mathbb{E}\left[f\left(X_{r}\right) \mid N_{r}\right]\right] \mathbb{E}\left[\mathbb{E}\left[g\left(X_{s}-X_{r}\right) \mid N_{s-r}\right]\right] \\
& =\mathbb{E}\left[f\left(X_{r}\right)\right] \mathbb{E}\left[g\left(X_{s}-X_{r}\right)\right] .
\end{aligned}
$$

2. Estacionariedade dos incrementos: Seja $0<r<s$ e $f$ uma função Borel limitada. Ao utilizar as observações feitas acima, é possível escrever:

$$
\begin{aligned}
\mathbb{E}\left[f\left(X_{s}-X_{r}\right)\right] & =\mathbb{E}\left[\mathbb{E}\left[f\left(\sum_{i=N_{r}+1}^{N_{s}} Y_{i}\right) \mid N_{t}, t \leq s\right]\right] \\
& =\mathbb{E}\left[\mathbb{E}\left[f\left(\sum_{i=1}^{N_{s}-N_{r}} Y_{N_{r}+i}\right) \mid N_{t}, t \leq s\right]\right] .
\end{aligned}
$$


Ao observar que o tamanho do saltos $\left\{Y_{i}\right\}_{i \geq 0}$ são independentes e identicamente distribuídos, e que o processo $N$ é estacionário por incrementos, tem-se:

$$
\begin{aligned}
\mathbb{E}\left[f\left(X_{s}-X_{r}\right)\right] & =\mathbb{E}\left[\mathbb{E}\left[f\left(\sum_{i=1}^{N_{s}-N_{r}} Y_{i}\right) \mid N_{t}, t \leq s\right]\right] \\
& =\mathbb{E}\left[\mathbb{E}\left[f\left(\sum_{i=1}^{N_{s-r}} Y_{i}\right) \mid N_{t}, t \leq s\right]\right]=\mathbb{E}\left[f\left(X_{s-r}\right)\right] .
\end{aligned}
$$

3. Continuidade estocástica: $X_{t}$ apresenta saltos apenas quando $N_{t}$ também apresenta. Desta maneira, pela Propriedade 4 da Proposição 1.6, tem-se que para $t>0$,

$$
\mathbb{P}\left(N_{s} \underset{s \rightarrow t}{\stackrel{s<t}{\longrightarrow}} N_{t}\right)=1 .
$$

Conseqüentemente, para qualquer $t>0$,

$$
\mathbb{P}\left(X_{s} \underset{s \rightarrow t}{\stackrel{s<t}{\longrightarrow}} X_{t}\right)=1 .
$$

Assim, convergência quase-certa garante convergência em probabilidade, que implica em continuidade estocástica.

Assim, qualquer função cadlag pode ser aproximada por uma função constante por partes. Isto indica que processos de Lévy, de uma maneira geral, podem ser aproximados por processos compostos de Poisson, o que possibilita um melhor entendimento dos processos de Lévy.

\section{Proposição 2.4 Função Característica de um Processo Composto de Poisson} Seja $\left\{X_{t}\right\}_{t \geq 0}$ um processo compensado de Poisson em $\mathbb{R}^{d}$. A sua função característica possui a seguinte representação:

$$
\mathbb{E}\left[\exp \left(i u . X_{t}\right)\right]=\exp \left\{t \lambda \int_{\mathbb{R}^{d}}(\exp (i u . x)-1) f(d x)\right\}, \forall u \in \mathbb{R}^{d},
$$

sendo que $\lambda$ representa a intensidade do salto e $f$ a distribuição do tamanho do salto.

Ao comparar a função característica de um processo composto de Poisson (2.4) com a do processo de Poisson (1.34), é possível observar que a v.e. composta de Poisson pode ser representada como uma superposição de processos independentes de Poisson com tamanhos de saltos distintos. A intensidade total de um processo de Poisson com tamanho de saltos no intervalo $[x ; x+d x]$ é determinada pela densidade $\lambda f(d x)$. 
Prova Ao condicionar a esperança em $N_{t}$ e representando a função característica de $f$ por $\hat{f}$, tem-se

$$
\begin{aligned}
\mathbb{E}\left[\exp \left(i u . X_{t}\right)\right] & =\mathbb{E}\left[\mathbb{E}\left[\exp \left(i u \cdot X_{t}\right) \mid N_{t}\right]\right]=\mathbb{E}\left[\hat{f}(u) \mid N_{t}\right]=\mathbb{E}\left[\hat{f}(u)^{N_{t}}\right] \\
& =\sum_{n=0}^{\infty} \frac{\exp (-\lambda t)(\lambda t)^{n} \hat{f}(u)^{n}}{n !}=\exp \{\lambda t(\hat{f}(u)-1)\} \\
& =\exp \left\{\lambda t\left(\mathbb{E}\left[\exp \left(i u \cdot X_{t}\right)\right]-1\right)\right\} \\
& =\exp \left\{\lambda t\left(\int_{\mathbb{R}^{d}} \exp (i u \cdot x) f(d x)-\int_{\mathbb{R}^{d}} f(d x)\right)\right\} \\
& =\exp \left\{\lambda t\left(\int_{\mathbb{R}^{d}}(\exp (i u \cdot x)-1) f(d x)\right)\right\} .
\end{aligned}
$$

No caso particular de um processo composto de Poisson unidimensional a função característica assume uma forma mais simples:

$$
\mathbb{E}\left[\exp \left(i u X_{t}\right)\right]=\exp \left\{t \lambda \int_{-\infty}^{\infty}(\exp (i u x)-1) f(d x)\right\}, \forall u \in \mathbb{R} .
$$

Ao introduzir uma medida $\nu(A)=\lambda f(A)$, é possível escrever a equação (2.4) da seguinte maneira:

$$
\mathbb{E}\left[\exp \left(i u . X_{t}\right)\right]=\exp \left\{t \int_{\mathbb{R}^{d}}(\exp (i u . x)-1) \nu(d x)\right\}, \forall u \in \mathbb{R}^{d} .
$$

A medida $\nu$ é denominada medida de Lévy referente ao processo $\left\{X_{t}\right\}_{t \geq 0}$. $\nu$ é uma medida positiva em $\mathbb{R}$, mas não é uma medida probabilística, pois $\int \nu(d x)=\lambda \neq 1$. A equação (2.5) é um caso particular da representação Lévy-Khinchin ([Teorema (2.1)].

\subsection{Medida de Salto do Processo Composto de Poisson}

Como foi apresentado anteriormente na Subseção 1.4.4, um processo cadlag, em particular, um processo composto de Poisson $X=\left\{X_{t}\right\}_{t \geq 0}$ em $\mathbb{R}^{d}$ pode ser associado a uma medida estocástica em $\mathbb{R}^{d} \times[0 ; \infty[$ que descreve os saltos do processo $X$, i.e, para conjunto mensurável $B \subset \mathbb{R}^{d} \times[0 ; \infty[$ tem-se

$$
J_{X}(B)=\#\left\{\left(t, X_{t}-X_{t-}\right) \in B\right\}
$$

Para qualquer conjunto mensurável $A \subset \mathbb{R}^{d}, J_{X}\left(\left[t_{1} ; t_{2}\right] \times A\right)$ enumeram-se os momentos de ocorrência dos saltos de $X$ no intervalo de tempo $t_{1}$ e $t_{2}$, para os saltos de 
tamanho pertencentes ao conjunto $A$. A seguinte proposição mostra que $J_{X}$ é uma medida estocástica de Poisson no sentido da Definição 1.14.

\section{Proposição 2.5 Medida de Salto de um Processo Composto de Poisson Seja} $\left\{X_{t}\right\}_{t \geq 0}$ um processo composto de Poisson com intensidade $\lambda$ e tamanho de salto com distribuição $f$. A medida de salto $J_{X}$ é uma medida estocástica de Poisson em $\mathbb{R}^{d} \times$ $[0 ; \infty[$ com intensidade da medida $\mu(d x \times d t)=\nu(d x) d t=\lambda f(d x) d t$.

Esta proposição sugere interpretar a medida de Lévy de um processo composto de Poisson como a média de saltos por unidade de tempo. Cabe ressaltar que esta interpretação pode ser generalizada para qualquer medida de Lévy de todos os processos de Lévy e não apenas do processo composto de Poisson, como segue:

Definição 2.4 Medida de Lévy Seja $X=\left\{X_{t}\right\}_{t \geq 0}$ um processo de Lévy em $\mathbb{R}^{d}$. A medida $\nu$ em $\mathbb{R}^{d}$, definida como

$$
\nu(A)=\mathbb{E}\left[\#\left\{t \in[0,1]: \Delta X_{t} \neq 0, \Delta X_{t} \in A\right\}\right], \quad A \in \mathcal{B}\left(\mathbb{R}^{d}\right),
$$

é denominada medida de Lévy do processo $X$, sendo que $\nu(A)$ é a quantidade esperada de saltos por unidade de tempo, cujos tamanhos pertencem ao conjunto $A$.

\section{Prova da Proposição 2.5}

1. Pela medida de salto apresentada pela equação (2.6) pode-se observar que $J_{X}$ é uma medida de valores inteiros. Inicialmente, é possível verificar que a distribuição de $J_{X}(B)$ possui distribuição de Poisson. É suficiente para provar esta propriedade para o conjunto $B=A \times\left[t_{1} ; t_{2}\right]$ para $A \in \mathcal{B}\left(\mathbb{R}^{d}\right)$.

2. Seja, $\left\{N_{t}\right\}_{t \geq 0}$ um processo de Poisson, que enumera a quantidade de saltos de $X$. Condicionada a trajetória de $N$, o tamanho dos saltos $Y_{i}$ são i.i.d. e $J_{X}\left(\left[t_{1} ; t_{2}\right] \times A\right)$ é a soma de $N_{t_{2}}-N_{t_{1}}$ variáveis estocásticas de Bernoulli i.i.d., que assumem valores 1 com probabilidade $f(A)$. Desta maneira,

$$
\begin{aligned}
\mathbb{E}\left[\exp \left\{i u J_{X}\left(\left[t_{1} ; t_{2}\right] \times A\right)\right\}\right] & =\mathbb{E}\left[\mathbb{E}\left[\exp \left\{i u J_{X}\left(\left[t_{1} ; t_{2}\right] \times A\right)\right\} \mid N_{t}, t \geq 0\right]\right] \\
& =\mathbb{E}\left[\mathbb{E}\left[\exp \left\{i u J_{X}\left(\left[t_{1} ; t_{2}\right] \times A\right)\right\} \mid N_{t_{2}}-N_{t_{1}}\right]\right]
\end{aligned}
$$


Dado que a função característica de uma v.e. distribuída por Bernoulli, com probabilidade do valor 1 ser igual a $f(A)$, é $\Phi(u)=1-f(A)+f(A) \exp (i u)$ e, $N_{t_{2}}-N_{t_{1}}$ é uma v.e. de Poisson com intensidade igual a $\lambda\left(t_{2}-t_{1}\right)$, então,

$$
\begin{aligned}
\mathbb{E}\left[\exp \left\{i u J_{X}\left(\left[t_{1} ; t_{2}\right] \times A\right)\right\}\right] & =\mathbb{E}\left[1-f(A)+f(A) \exp (i u) \mid N_{t_{2}}-N_{t_{1}}\right] \\
& =\mathbb{E}\left[\{1-f(A)+f(A) \exp (i u)\}^{\left(N_{t_{2}}-N_{t_{1}}\right)}\right] \\
& =\exp \left\{\lambda\left(t_{2}-t_{1}\right) f(A)(\exp (i u)-1)\right\} .
\end{aligned}
$$

Como pode ser observado pela função característica acima, $J_{X}\left(\left[t_{1} ; t_{2}\right] \times A\right)$ é uma variável de Poisson com parâmetro $\lambda\left(t_{2}-t_{1}\right) f(A)$.

3. Seja $A$ e $B$ dois conjuntos mensuráveis desconectados. Primeiro, é possível mostrar que se $A$ e $B$ são dois conjuntos Borel em $\mathbb{R}^{d}$ desconectados, então, $J_{X}\left(\left[t_{1} ; t_{2}\right] \times A\right)$ e $J_{X}\left(\left[t_{1} ; t_{2}\right] \times B\right)$ são independentes. Condicionado na trajetória de $N$, a expressão iu $J_{X}\left(\left[t_{1} \times t_{2}\right] \times A\right)+i \nu J_{X}\left(\left[t_{1} \times t_{2}\right] \times B\right)$ é a soma de $N_{t_{2}}-N_{t_{1}}$ variáveis independentes que assumem valores:

$$
\begin{array}{ll}
\text { iu } & \text { com probabilidade } f(A) ; \\
i \nu & \text { com probabilidade } f(B) ; \\
0 & \text { com probabilidade } 1-f(A)-f(B) .
\end{array}
$$

Ao proceder de maneira análoga a parte 1 desta prova, é possível fatorar a função característica da seguinte maneira:

$$
\begin{aligned}
\mathbb{E}[\exp & \left.\left\{i u J_{X}\left(\left[t_{1} \times t_{2}\right] \times A\right)+i \nu J_{X}\left(\left[t_{1} \times t_{2}\right] \times B\right)\right\}\right] \\
& =\mathbb{E}\left[\mathbb{E}\left[\exp \left\{i u J_{X}\left(\left[t_{1} \times t_{2}\right] \times A\right)+i \nu J_{X}\left(\left[t_{1} \times t_{2}\right] \times B\right)\right\}\right]^{\left(N_{t_{2}}-N_{t_{1}}\right)}\right] \\
& =\mathbb{E}\left[\{1+f(A)(\exp (i u)-1)+f(B)(\exp (i \nu)-1)\}{ }^{\left(N_{t_{2}}-N_{t_{1}}\right)}\right] \\
& =\exp \left\{\lambda\left(t_{2}-t_{1}\right)[f(A)(\exp (i u)-1)+f(B)(\exp (i \nu)-1)]\right\} \\
& =\exp \left\{\lambda\left(t_{2}-t_{1}\right) f(A)(\exp (i u)-1)\right\} \exp \left\{\lambda\left(t_{2}-t_{1}\right) f(B)(\exp (i \nu)-1)\right\} \\
& =\mathbb{E}\left[\exp \left\{i u J_{X}\left(\left[t_{1} \times t_{2}\right] \times A\right)\right\}\right] \mathbb{E}\left[\exp \left\{i \nu J_{X}\left(\left[t_{1} \times t_{2}\right] \times B\right)\right\}\right] .
\end{aligned}
$$

Esta propriedade das medidas estocásticas de Poisson é denominada propriedade aditiva. Segundo, sejam $\left[t_{1} ; t_{2}\right]$ e $\left[s_{1} ; s_{2}\right]$ dois conjuntos desconectados. A independência de $J_{X}\left(\left[t_{1} \times t_{2}\right] \times A\right)$ e $J_{X}\left(\left[s_{1} \times s_{2}\right] \times B\right)$ é conseqüência da inde- 
pendência dos incrementos do processo $X$. Desta maneira, a independência das medidas de salto para qualquer quantidade de conjuntos desconectados em $\left[0 ; \infty\left[\times \mathbb{R}^{d}\right.\right.$ segue da propriedade aditiva das medidas $J_{X}$ e do fato de que os métodos usados nesta prova podem ser estendidos a uma quantidade enumerável de conjuntos.

A Proposição 2.5 implica que cada processo composto de Poisson pode ser representado da seguinte maneira:

$$
X_{t}=\sum_{s \in[0 ; t]} \Delta X_{s}=\int_{[0 ; t] \times \mathbb{R}^{d}} x J_{X}(d s \times d x),
$$

sendo que $J_{X}$ é uma medida estocástica de Poisson com intensidade igual a medida $\nu(d x) d t$. Este é um caso particular da decomposição de Lévy-Itô para os processos de Lévy [Proposicão 2.7]. Neste caso, só é possível reescrever o processo $X$ como a soma de saltos. Assim, um processo composto de Poisson tem quase-certamente uma quantidade enumerável de saltos no intervalo $[0 ; t]$, e a integral estocástica na equação (2.8) é uma soma finita, não apresentando problemas de convergência.

O seguinte lema, que é muito parecido com a Proposição 1.3, é de grande utilidade para calcular as quantidades relacionadas as medidas estocásticas de Poisson.

Lema 2.1 Seja M uma medida estocástica de Poisson com intensidade da medida $\mu$ e A um conjunto mensurável, tal que $0<\mu(A)<\infty$. Assim, as seguintes medidas estocásticas em A têm distribuição condicionada em $M(A)$ :

1. $\left.M\right|_{A}$ é a restrição de $M$ em $A$.

2. $\widehat{M}_{A}$ é definida como $\widehat{M}_{A}(B)=\#\left\{X_{i} \in B\right\}$ para todo subconjunto mensurável $B \in A$, tal que $X_{i}, i=1, \ldots, M(A)$ são independentes e distribuídas em A com distribuição $\frac{\mu(d x)}{\mu(A)}$. Em outras palavras, $\widehat{M}_{A}$ é a medida de contagem de $M(A)$ pontos estocásticos independentes e identicamente distribuídos em A. 
Prova Seja $B_{1}, \cdots, B_{k}$ subconjuntos mensuráveis desconectados de $A$, $\tilde{B}=A \backslash\left(B_{1} \cup \cdots \cup B_{k}\right)$ e $\tilde{n}=n-\sum_{i=1}^{k} n_{i}$. Assim,

$$
\begin{aligned}
& \mathbb{P}\left[M\left(B_{1}\right)=n_{1}, \ldots, M\left(B_{k}\right)=n_{k} \mid M(A)=n\right] \\
& \quad=\frac{\mathbb{P}\left[M\left(B_{1}\right)=n_{1}, \ldots, M\left(B_{k}\right)=n_{k}, M(\tilde{B})=\tilde{n}\right]}{\mathbb{P}[M(A)=n]} .
\end{aligned}
$$

Pela propriedade de independência dos incrementos de um processo de Poisson (Propriedade 8 da Proposição 1.6) é possível escrever o resultado acima da seguinte maneira:

$$
\begin{aligned}
& =\left(\prod_{i=1}^{k} \frac{\mu\left(B_{i}\right)^{n_{i}} \exp \left\{\mu\left(B_{i}\right)\right\}}{n_{i} !}\right)\left(\frac{\mu(\tilde{B})^{\tilde{n}} \exp \{\mu(\tilde{B})\}}{\tilde{n} !}\right)\left(\frac{\mu(A)^{n} \exp \{\mu(A)\}}{n !}\right)^{-1} \\
& =\frac{n !}{n_{1} ! \cdots n_{k} ! \tilde{n} !} \frac{\mu\left(B_{1}\right)^{n_{1}} \cdots \mu\left(B_{k}\right)^{n_{k}} \mu(\tilde{B})^{\tilde{n}}}{\mu(A)^{n}} \frac{\exp \left\{\mu\left(B_{1}\right)+\cdots \mu\left(B_{k}\right)+\mu(\tilde{B})\right\}}{\exp \{\mu(A)\}} \\
& =\frac{n !}{n_{1} ! \cdots n_{k} ! \tilde{n} !} \frac{\mu\left(B_{1}\right)^{n_{1}} \cdots \mu\left(B_{k}\right)^{n_{k}} \mu(\tilde{B})^{\tilde{n}}}{\mu(A)^{\left(n_{1}+\cdots+n_{k}+\tilde{n}\right)}} \frac{\exp \{\mu(A)\}}{\exp \{\mu(A)\}} \\
& =\frac{n !}{n_{1} ! \cdots n_{k} ! \tilde{n} !}\left(\frac{\mu\left(B_{1}\right)}{\mu(A)}\right)^{n_{1}} \cdots\left(\frac{\mu\left(B_{k}\right)}{\mu(A)}\right)^{n_{k}}\left(\frac{\mu(\tilde{B})}{\mu(A)}\right)^{\tilde{n}},
\end{aligned}
$$

que é igual a distribuição conjunta de $\widehat{M}_{A}\left(B_{i}\right) \cdots \widehat{M}_{A}\left(B_{k}\right)$. Utilizando a propriedade aditiva (p.61) destas medidas é possível verificar que $B_{1}, \ldots, B_{k}$ não necessitam ser obrigariamente subconjuntos desconectados.

Uma aplicação do Lema 2.1, segue do seguinte resultado:

\section{Proposição 2.6 Fórmula Exponencial de uma Medida Estocástica de Poisson}

Seja M uma medida estocástica de Poisson com intensidade da medida $\mu$. Assim, a fórmula

$$
\mathbb{E}\left[\exp \left\{\int_{B} f(s) M(d s)\right\}\right]=\exp \left\{\int_{B}(\exp \{f(s)\}-1) \mu(d s)\right\}
$$

vale para qualquer conjunto mensurável $B$, tal que, $\mu(B)<\infty$ e todas as funções $f$ que atendem a condição $\int_{B} \exp \{f(s)\} \mu(d s)<\infty$.

Prova Ao representar o processo composto de Poisson por uma medida $X_{f}(B)=\int_{B} f(s) M(d s)$, com intensidade $\mu(B)$ é possível desenvolver a equação 
(2.9) da seguinte maneira:

$$
\begin{aligned}
\mathbb{E}\left[\exp \left\{\int_{B} f(s) M(d s)\right\}\right] & =\mathbb{E}\left[\exp \left\{X_{f}(B)\right\}\right] \\
& =\mathbb{E}\left[\mathbb{E}\left[\exp \left\{X_{f}(B)\right\} \mid M(B)\right]\right] \\
& =\mathbb{E}\left[\exp \left\{\int_{B}(\exp \{f(s)\}-1) \mu(d s)\right\} \mid M(B)\right] \\
& =\exp \left\{\int_{B}(\exp \{f(s)\}-1) \frac{\mu(d s)}{\mu(B)}\right\} \mid M(B)=\mu(B) \\
& =\left(\exp \left\{\int_{B}(\exp \{f(s)\}-1) \frac{\mu(d s)}{\mu(B)}\right\}\right) \\
& =\exp \left\{\mu(B) \int_{B}(\exp \{f(s)\}-1) \frac{\mu(d s)}{\mu(B)}\right\} \\
& =\exp \left\{\int_{B}(\exp \{f(s)\}-1) \mu(d s)\right\} .
\end{aligned}
$$

Adiante, será possível verificar que as condições $\mu(B)<\infty$ e $\int_{B} \exp \{f(x)\} \mu(d x)<\infty$ não são necessárias para garantir o resultado apresentado na equação (2.9), pois é suficiente apenas atender a condição $\int_{B}|\exp \{f(x)\}-1| \mu(d x)<\infty$. A Proposição 2.6 permite estabelecer uma correspondência unívoca entre os processos compostos de Poisson e as medidas estocásticas de Poisson com intensidade da medida $\nu(d x) d t$, para $\nu$ finita. Seja $\nu$ uma medida finita em $\mathbb{R}^{d}$ e $M$ uma medida estocástica de Poisson em $\mathbb{R}^{d} \times[0 ; \infty[$ com intensidade da medida $\nu(d x) d t$. Ao utilizar a Proposição 2.6 é possível verificar que a equação (2.8) define um processo composto de Poisson com medida de Lévy $\nu$.

\subsection{Atividade Infinita do Processo de Lévy}

Como foi observado, qualquer processo de Lévy $X_{t}^{0}$ constante por partes pode ser representado pela equação (2.8) dado uma medida estocástica de Poisson com intensidade da medida $\nu(d x) d t$ onde $\nu$ é uma medida finita, definida como:

$$
\nu(A)=\mathbb{E}\left[\#\left\{t \in[0 ; 1]: \Delta X_{t}^{0} \neq 0, \Delta X_{t}^{0} \in A\right\}\right], A \in \mathcal{B}\left(\mathbb{R}^{d}\right) .
$$


Dado um MB com t.d. $\gamma t+B_{t}$, indepentende de $X^{0}$, a soma de $X_{t}^{0}+\gamma t+B_{t}$ definite outro processo de Lévy, que pode ser decomposto como:

$$
X_{t}=\gamma t+B_{t}+\sum_{s \in[0 ; t]} \Delta X_{s}^{0}=\gamma t+B_{t}+\int_{[0 ; t] \times \mathbb{R}^{d}} x J_{X}(d s \times d x),
$$

sendo que $J_{X}$ é a medida estocástica de Poisson em $\left[0 ; \infty\left[\times \mathbb{R}^{d}\right.\right.$ com intensidade $\nu(d x) d t$.

Dado um processo de Lévy $X_{t}$ é possível definir uma medida de Lévy $\nu$ como apresentado acima (2.10), sendo que $\nu(A)$ ainda será finita para qualquer conjunto compacto $A$, tal que, $0 \notin A$, i.e., se esta condição não for verdadeira, o processo poderá apresentar uma quantidade infinita de saltos em $[0 ; T]$, que contradiz a propriedade cadlag do processo. Logo, $\nu$ define uma medida de Radon em $\mathbb{R}^{d} \backslash\{0\}$. Entretanto, $\nu$ não é necessariamente uma medida finita, pois a restrição acima ainda permite uma explosão em 0 , e $X$ pode ter uma quantidade infinita de saltos em $[0 ; T]$. Nesta caso, a soma dos saltos se comporta como uma série infinita e para que haja convergência é necessário impor algumas condições sob a medida $\nu$, sob as quais é possível decompor $X$ da seguinte maneira:

Proposição 2.7 Decomposição de Lévy-Itô $\quad$ Seja $X=\left\{X_{t}\right\}_{t \geq 0}$ um processo de Lévy em $\mathbb{R}^{d}$ e $\nu$ a sua medida de Lévy [Definição 2.4], então:

1. $\nu$ é uma medida de Radon em $\mathbb{R}^{d} \backslash\{0\}$ e

$$
\int_{|x| \leq 1}|x|^{2} \nu(d x)<\infty \quad \int_{|x| \geq 1} \nu(d x)<\infty .
$$

2. A medida de salto de $X$, representada por $J_{X}$, é uma medida estocástica de Poisson em $\nu(d x) d t$.

3. Existe um vetor $\gamma$ e um $M B$ d-dimensional $\left\{B_{t}\right\}_{t \geq 0}$ com matriz de covariância $\Sigma$, tal que,

$$
X_{t}=\gamma t+B_{t}+X_{t}^{l}+\lim _{\varepsilon \downarrow 0} \tilde{X}_{t}^{\varepsilon}
$$


sendo que:

$$
\begin{aligned}
X_{t}^{l} & =\int_{|x| \geq 1, s \in[0, t]} x J_{X}(d s \times d x) \\
\tilde{X}_{t}^{\varepsilon} & \triangleq \int_{\varepsilon \leq|x|<1, s \in[0 ; t]} x \tilde{J}_{X}(d s \times d x) \\
& =\int_{\varepsilon \leq|x|<1, s \in[0 ; t]} x\left\{J_{X}(d s \times d x)-\nu(d x) d s\right\} .
\end{aligned}
$$

Os termos da equação (2.11) são independentes e o último termo é convergente de maneira uniforme em $t \in[0 ; T]$ quase-certamente.

A decomposição de Lévy-Itô garante que para qualquer processo de Lévy existe um vetor $\gamma$, uma matriz definida positiva $\Sigma$ e uma medida positiva $\nu$ que determina uma única distribuição. $\mathrm{O}$ tripé $(\Sigma, \nu, \gamma)$ é denominado tripé característico ou tripé de Lévy de um processo $X_{t}$.

Dado a importância deste resultado, é importante interpretar o significado da equação (2.11). Primeiro, $\gamma t+B_{t}$ é um processo gaussiano de Lévy contínuo e, qualquer processo gaussiano de Lévy contínuo pode ser escrito desta maneira, sendo que os dois parâmetros: a t.d. $\gamma$ e a matriz de covariância $\Sigma$ do MB.

Os outros dois termos são processos descontínuos que incorporam os saltos de $X_{t}$, sendo descritos pela medida de Lévy $\nu$. A condição $\int_{|y| \geq 1} \nu(d y)<\infty$ significa que $X$ possui uma quantidade finita de saltos com valores absolutos maiores que 1 . Assim, a soma:

$$
X_{t}^{l}=\sum_{0 \leq s \leq t}^{\left|\Delta X_{s}\right| \geq 1} \Delta X_{s}
$$

contém quase-certamente um número finito de termos e $X_{t}^{l}$ é um processo composto de Poisson. Não há nada de especial em relação a $\Delta X=1$. Para qualquer $\varepsilon>0$, a soma de saltos com amplitude entre $\varepsilon$ e 1 :

$$
X_{t}^{\varepsilon}=\sum_{0 \leq s \leq t}^{1>\left|\Delta X_{s}\right|>\varepsilon} \Delta X_{s}=\int_{\varepsilon \leq|x|<1, s \in[0 ; t]} x J_{X}(d s \times d x)
$$

é novamente um processo composto de Poisson bem comportado. Entretanto, neste caso, a medida $\nu$ do processo composto de Poisson pode apresentar singulariedade no ponto zero, i.e., pode existir uma quantidade infinita de pequenos saltos, e a respectiva soma destes saltos pode não necessariamente convergir. Esta definição previne que 
$\varepsilon$ seja 0 na expressão (2.12). Para garantir a convergência, é necessário centralizar este termo, i.e., substituir a integral dos saltos por uma versão compensada, como foi definida na Seção 1.3.4:

$$
X_{t}^{\varepsilon}=\int_{\varepsilon \leq|x|<1, s \in[0 ; t]} x \tilde{J}_{X}(d s \times d x)
$$

que, pela Proposição 1.10 é uma martingale. Enquanto $X^{\varepsilon}$ pode ser interpretada como a superposição de uma quantidade infinita de processos de Poisson, $\tilde{X}_{t}^{\varepsilon}$ deve ser entendida como uma superposição de uma quantidade infinita de processos compensados de Poisson, i.e., processos de Poisson centrados para os quais existe algum tipo de limitecentral para que seja possível mostrar a convergência do termo.

Uma implicação importante referente à decomposição de Lévy-Itô é que para cada processo de Lévy existe uma combinação de MB com t.d. e possivelmente a soma de uma quantidade infinita de processos compostos de Poisson. Isto significa que cada processo de Lévy pode ser aproximado, dado uma precisão arbitrária, por um processo de salto-difusão, i.e., pela soma de um MB com t.d. e um processo composto de Poisson, o que é muito útil, tanto na teoria como na prática. A Figura 2.2 ilustra um típico processo de salto-difusão.

Prova da Decomposição de Lévy-Itô Incialmente, construa uma medida estocástica de Poisson $J_{X}$ em $[0 ; t] \times \mathbb{R}^{d}$ a partir dos saltos de $X=\left\{X_{t}\right\}_{t \geq 0}$. Como $X$ é cadlag, para qualquer valor positivo $\varepsilon$, o conjunto $\left\{t:\left|X_{t}-X_{t-}\right| \geq \varepsilon\right\}$ é finito e a medida estocástica de Poisson, referente a qualquer conjunto que não contenha o zero, pode ser construída pela utilização da Proposição 2.5. A intensidade de $J_{X}$ é homogênea e igual a $\nu(d x) d t$. Até o final desta prova, é possível supor que, sem perda de generalidade, que todos os saltos de $X$ são menores que 1 em valor absoluto. Dado o seguinte lema [Kallenberg (2002), Lema 15.6, p.288]:

Lema 2.2 Seja $\left\{X_{t}, Y_{t}\right\}_{t \geq 0}$ um processo de Lévy. Se $Y$ é um processo composto de Poisson e, $X$ e $Y$ nunca apresentam saltos no mesmo instante, i.e., $\Delta X_{t} \Delta Y_{t}=0$, então, estes processos são independentes. 

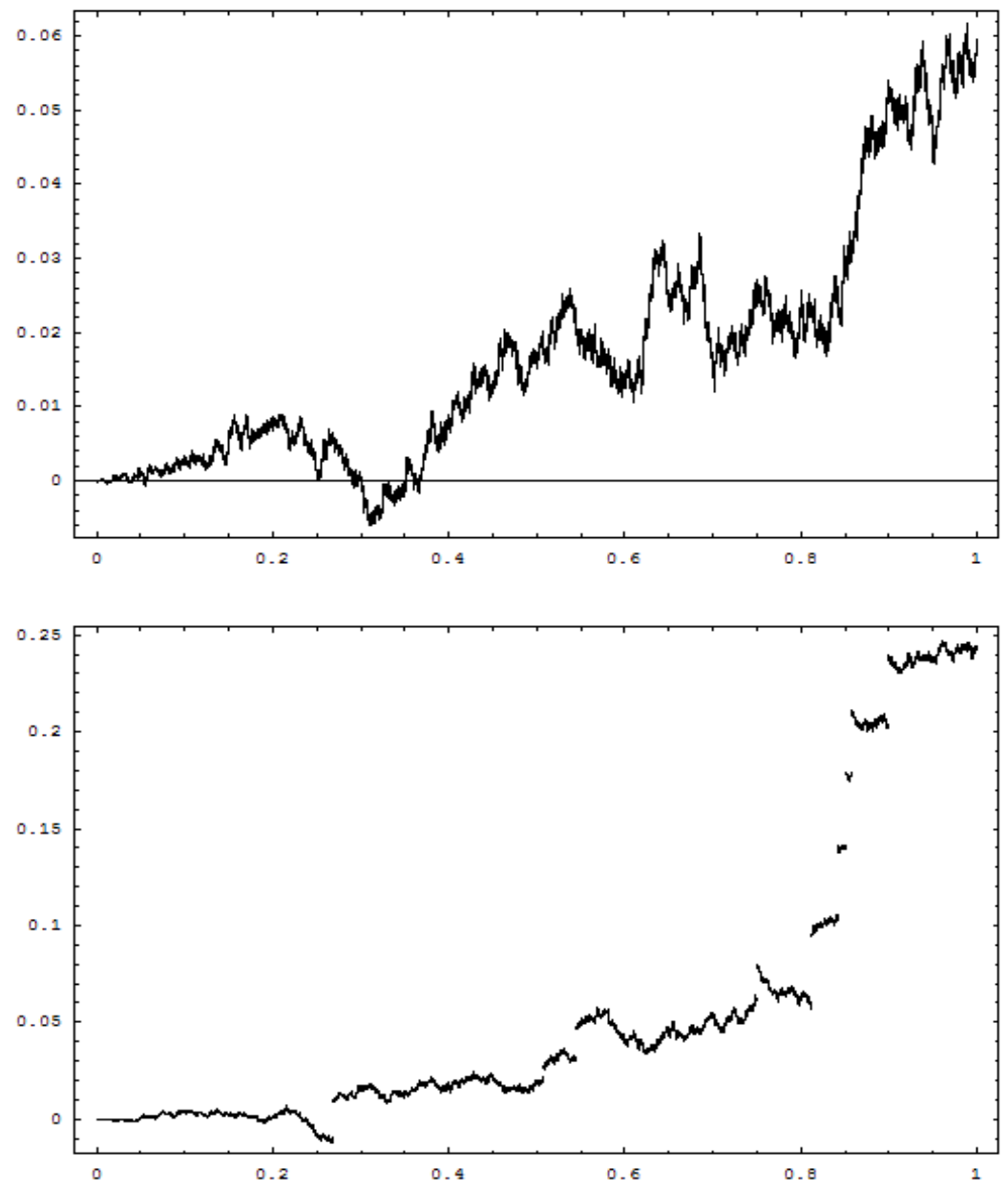

Figura 2.2. A figura a acima mostra uma trajetória típica de um movimento browniano com tendência determinística e, a figura abaixo mostra uma trajetória típica de um processo de salto-difusão. 
O Lema 2.2 em conjunto com a Proposição 2.6 permite provar que a medida de Lévy $\nu$ satifaz a seguinte condição:

$$
\int\left(|x|^{2} \wedge 1\right) \nu(d x)<\infty
$$

Como uma medida de Lévy de qualquer conjunto fechado não contendo o zero é finito, é suficiente provar que para um $\delta>0, \int_{|x| \leq \delta}|x|^{2} \nu(d x)<\infty$. Seja $X_{t}^{\varepsilon}$ um processo de Lévy definido pelo resultado (2.13), e $\left\{X_{t}\right\}_{t \geq 0}$ outro processo de Lévy qualquer, e $R_{t}^{\varepsilon}=X_{t}-X_{t}^{\varepsilon}$, logo, $\left\{R_{t}^{\varepsilon}, X_{t}^{\epsilon}\right\}$ também será um processo de Lévy. É possível observar que para um $u$ e $t,\left|\mathbb{E}\left[\exp \left\{i u X_{t}\right\}\right]\right|>0$. Ao aplicar o Lema 2.2 obtém-se que $\left\{X_{t}^{\varepsilon}\right\}$ e $\left\{R_{t}^{\varepsilon}\right\}$ são independentes, i.e.,

$$
\mathbb{E}\left[\exp \left\{i u X_{t}\right\}\right]=\mathbb{E}\left[\exp \left\{i u R_{t}^{\varepsilon}\right\}\right] \mathbb{E}\left[\exp \left\{i u X_{t}^{\varepsilon}\right\}\right] .
$$

Isto significa que $\left|\mathbb{E}\left[\exp \left\{i u X_{t}^{\varepsilon}\right\}\right]\right|$ possui um limite inferior positivo que não depende de $\varepsilon$. Pela Proposição 2.6 isto é equivalente a

$$
\begin{aligned}
\mid \exp & \left\{t \int_{|x| \geq \varepsilon}(\exp \{i u x\}-1) \nu(d x)\right\} \mid \\
& =\left|\exp \left\{t \int_{|x| \geq \varepsilon}\{\cos (u x)+i \sin (u x)-1\} \nu(d x)\right\}\right| \geq C
\end{aligned}
$$

Ao considerar apenas a parte real, i.e.,

$$
\begin{gathered}
\operatorname{Re}\left\{\left|\exp \left\{t \int_{|x| \geq \varepsilon}\{\cos (u x)+i \sin (u x)-1\} \nu(d x)\right\}\right|\right\} \\
\quad=\left|\exp \left\{t \int_{|x| \geq \varepsilon}\{\cos (u x)-1\} \nu(d x)\right\}\right| \geq C .
\end{gathered}
$$

Dado que a função $f(x) \triangleq|x|$, possui concavidade positiva, i.e., $d^{2} f(x) / d x^{2} \geq 0$, e que $g(x) \triangleq \int_{|x| \geq \varepsilon}\{\cos (u x)-1\} \nu(d x)$, então, pela desilguadade de Jensen tem-se que $g(\exp \{t|x|\}) \geq|\exp \{t g(x)\}|$ :

$$
\begin{aligned}
& \exp \left\{t\left|\int_{|x| \geq \varepsilon}\{\cos (u x)-1\} \nu(d x)\right|\right\} \\
& \quad \geq\left|\exp \left\{t \int_{|x| \geq \varepsilon}\{\cos (u x)-1\} \nu(d x)\right\}\right| \geq C \\
& \left|\int_{|x| \geq \varepsilon}\{\cos (u x)-1\} \nu(d x)\right| \geq \frac{\ln C}{t} .
\end{aligned}
$$


Dado que $\int|f(d x)| \geq\left|\int f(d x)\right|$, tem-se que

$$
\begin{aligned}
& \int_{|x| \geq \varepsilon}|\cos (u x)-1| \nu(d x) \geq\left|\int_{|x| \geq \varepsilon}\{\cos (u x)-1\} \nu(d x)\right| \geq \frac{\ln C}{t} \\
& \int_{|x| \geq \varepsilon}|\cos (u x)-1| \nu(d x) \geq \frac{\ln C}{t} \geq-\int_{|x| \geq \varepsilon}|\cos (u x)-1| \nu(d x) \\
& \int_{|x| \geq \varepsilon}|\cos (u x)-1| \nu(d x) \leq \tilde{C}
\end{aligned}
$$

sendo que $\tilde{C}=-t^{-1} \ln C<\infty$. Como $1-\cos (u x) \geq 0$, tem-se que $|-\{1-\cos (u x)\}|=|1-\cos (u x)|$, i.e.,

$$
\int_{|x| \geq \varepsilon}|1-\cos (u x)| \nu(d x)<\infty .
$$

Adicionalmente, quando $\varepsilon \longrightarrow 0$ tem-se o resultado (2.14), i.e.,

$$
\lim _{\varepsilon \downarrow 0} \int_{|x| \geq \varepsilon}|1-\cos (u x)| \nu(d x)=\int_{\mathbb{R}^{d}}\left(|x|^{2} \wedge 1\right) \nu(d x)
$$

Agora é necessário mostrar a convergência de $\tilde{X}_{t}^{\varepsilon}$. Considere a seqüência $\left\{\varepsilon_{n}\right\} \downarrow$ 0 e $Y_{n}=\tilde{X}_{t}^{\varepsilon_{n}+1}-\tilde{X}_{t}^{\varepsilon_{n}}$. Todas as variáveis $Y_{i}$ possuem esperança zero e estão sujeitas a condição (2.14), o que garante que $\sum \mathbb{V}\left[Y_{i}\right]<\infty$. Assim, pelo Teorema do critério de três-séries de Kolmogorov-Lévy [Kallenberg (2002), Teorema 4.18, p.71], $\tilde{X}_{t}^{\varepsilon}$ converge quase-certamente quando $\varepsilon \longrightarrow 0$. Ao aplicar o Lema da máxima desilgualdade de Komolgorov [Kallenberg (2002), Teorema 4.15, p.69] é possível verificar que esta convergência é linear em $t$.

Para completar a prova, considere um processo $X_{t}^{c}=X_{t}-\lim \tilde{X}_{t}^{\varepsilon}$, que é um processo de Lévy independente de $\tilde{X}_{t}^{\varepsilon}$ [Lema 2.2]. Este processo é contínuo, pois $\tilde{X}_{t}^{\varepsilon}$ converge de maneira uniforme em $t$, assim, é possível intercambiar o operador lim. Finalmente, pelo Teorema do Limite-Central de Feller-Lévy [Kallenberg (2002), Teorema 5.15, p.93] implica que o processo $X_{t}^{c}$ é gaussiano (distribuição gaussiana dos incrementos).

O conhecimento da estrutura das trajetórias de um processo de Lévy permite obter, sem muito esforço adicional, um outro resultado fundamental, i.e., a função característica de um processo de Lévy pode ser representada pelo tripé característico $(\Sigma, \nu, \gamma)$. 
Teorema 2.1 Representação de Lévy-Khinchin Seja $\left\{X_{t}\right\}_{t \geq 0}$ um processo de Lévy em $\mathbb{R}^{d}$ com tripé característico $(\Sigma, \nu, \gamma)$. Assim,

$$
\mathbb{E}\left[\exp \left(i z \cdot X_{t}\right)\right]=\exp \{t \psi(z)\}, \quad z \in \mathbb{R}^{d}
$$

sendo que

$$
\psi(z)=-\frac{1}{2} z \cdot \Sigma z+i \gamma \cdot z+\int_{\mathbb{R}^{d}}\left(\exp \left\{i z \cdot x-1-i z \cdot x 1_{\{|x| \leq 1\}}\right\}\right) \nu(d x) .
$$

Para um processo de Lévy de valores reais, a fórmula (2.15) assume a forma

$$
\mathbb{E}\left[\exp \left(i z X_{t}\right)\right]=\exp \{t \psi(z)\}, \quad z \in \mathbb{R}
$$

sendo que

$$
\psi(z)=-\frac{1}{2} \Sigma z^{2}+i \gamma z+\int_{-\infty}^{\infty}\left(\exp \left\{i z x-1-i z x 1_{\{|x| \leq 1\}}\right\}\right) \nu(d x) .
$$

Uma versão equivalente à representação de Lévy-Khinchin pode ser obtida desprezando (truncando) os saltos maiores que um número arbitrário $\varepsilon$ :

$$
\psi(z)=-\frac{1}{2} z \cdot \Sigma z+i \gamma^{\varepsilon} \cdot z+\int_{\mathbb{R}^{d}}\left(\exp \left\{i z \cdot x-1-i z \cdot x 1_{\{|x| \leq \varepsilon\}}\right\}\right) \nu(d x),
$$

sendo que

$$
\gamma^{\varepsilon}=\gamma+\int_{\mathbb{R}^{d}} x\left(1_{\{|x| \leq \varepsilon\}}-1_{\{|x| \leq 1\}}\right) \nu(d x)
$$

De uma maneira geral, para cada função limitada mensurável $g: \mathbb{R}^{d} \longrightarrow \mathbb{R}$ que satisfaça $g(x)=1+o(|x|)$ a medida que $x \longrightarrow 0$ e $g(x)=O(1 /|x|)$ a medida que $x \longrightarrow \infty$, é possível escrever

$$
\psi(z)=-\frac{1}{2} z \cdot \Sigma z+i \gamma^{g} \cdot z+\int_{\mathbb{R}^{d}}(\exp \{i z \cdot x\}-1-i z \cdot x g(x)) \nu(d x) .
$$

A função $g$ é denominada função de truncamento e o tripé característico $\left(\Sigma, \nu, \gamma^{g}\right)$ é denominado tripé característico de $X$ em relação à função de truncamento $g$. Escolhas diferentes de $g$ não afetam $\Sigma$ e $\nu$ que são parâmetros intrínsecos de um processo de Lévy. Entretanto, $\gamma$ depende da função de truncamento escolhida, assim, deve-se evitar dizer que $\gamma$ é a t.d. do processo. É possível escolhar entre várias funções de truncamento. A função de truncamento utilizada por Paul Lévy foi $g(x)=\frac{1}{1+|x|^{2}}$, enquanto que a função indicadora $1_{\{|x| \leq 1\}}$ parece ser a mais utilizada. Os resultados que serão desenvolvidos consideram a função de truncamento $1_{\{|x| \leq 1\}}$. 
Se uma medida de Lévy satisfaz a condiçaão $\int_{|x| \geq 1}|x| \nu(d x)<\infty$, então, não há necessidade de truncar os grandes saltos e pode-se usar a forma simplificada:

$$
\psi(z)=-\frac{1}{2} z \cdot \Sigma z+i \gamma_{c} \cdot z+\int_{\mathbb{R}^{d}}(\exp \{i z \cdot x-1-i z \cdot x\}) \nu(d x) .
$$

Neste caso, é possível mostrar que $\mathbb{E}\left[X_{t}\right]=\gamma_{c} t$ e $\gamma_{c}$ é denominada o centro do processo $\left\{X_{t}\right\}_{t \geq 0}$, e $\gamma_{c}$ relaciona-se com $\gamma$ por meio da função $\gamma_{c}=\gamma+\int_{|x| \geq 1} x \nu(d x)$.

Prova do Teorema 2.1 Pela Decomposição de Lévy-Itô [Proposição 2.7], é possível mostrar que para cada $t$, a v.e. $X_{t}^{c}+X_{t}^{l}+X_{t}^{\varepsilon}$ converge quase-certamente para $X_{t}$, quando $\varepsilon \longrightarrow 0$. Dado que convergência quase-certa implica convergência em distribuição, a função característica de $X_{t}^{c}+X_{t}^{l}+X_{t}^{\varepsilon}$ converge para a função característica de $X_{t}$. Desta maneira, como $X_{t}^{c}, X_{t}^{l}, X_{t}^{\varepsilon}$ são independentes, tem-se

$$
\begin{aligned}
& \mathbb{E}\left[\exp \left\{i z .\left(X_{t}^{c}+X_{t}^{l}+X_{t}^{\varepsilon}\right)\right\}\right]=\mathbb{E}\left[\exp \left\{i z \cdot X_{t}^{c}\right\}\right] \mathbb{E}\left[\exp \left\{i z \cdot X_{t}^{l}\right\}\right] \mathbb{E}\left[\exp \left\{i z \cdot X_{t}^{\varepsilon}\right\}\right] \\
& \quad=\exp \left\{-\frac{1}{2} z \cdot \Sigma z+i t \gamma \cdot z\right\} \times\left\{t \int_{|x| \geq 1}(\exp \{i z \cdot x\}-1) \nu(d x)\right\} \\
& \quad \times \exp \left\{t \int_{\varepsilon \leq|x| \leq 1}(\exp \{i z \cdot x\}-1-i z \cdot x) \nu(d x)\right\} .
\end{aligned}
$$

Quando $\varepsilon \longrightarrow 0$, tem-se

$$
\begin{aligned}
& \mathbb{E}\left[\exp \left\{i z \cdot X_{t}\right\}\right]= \\
& \quad \exp \left\{-\frac{1}{2} z \cdot \Sigma z+i t \gamma \cdot z+t \int_{\mathbb{R}^{d}}\left(\exp \{i z \cdot x\}-1-i z \cdot x_{|x| \leq 1}\right) \nu(d x)\right\} .
\end{aligned}
$$

Quando $\nu\left(\mathbb{R}^{d}\right)=\infty$ (caso de atividade infinita), o conjunto dos instantes de tempo dos saltos de cada trajetória de um processo de Lévy é infinitamente enumerável e denso em $[0 ; \infty[$. A enumeração segue diretamente do fato dos caminhos serem cadlag. Para provar que o conjunto de instantes de tempo é denso em $[0 ; \infty[$, considere um intervalo de tempo $[a ; b] \mathrm{e}$

$$
\begin{gathered}
\varepsilon(n)=\sup \left\{r: \int_{|x| \geq r} \nu(d x) \geq n\right\}, \\
Y_{n}=\int_{\varepsilon(n) \leq|x| \leq \varepsilon(n-1), t \in[a ; b]} J_{X}(d x \times d t) .
\end{gathered}
$$

Assim, se a medida de Lévy atribui massa zero (sem átomos) para qualquer conjunto finito de pontos e $Y_{i}$ são variáveis estocásticas de Poisson independentes e identicamente distribuídas, então, o número total de saltos no intervalo de tempo $[a ; b]$ é igual 
a $\sum_{i=1}^{\infty} Y_{i}$ e, que pela lei dos grandes números é infinito quase-certamente. Esta observação vale para qualquer intervalo de tempo $[a ; b]$ não vazio, isto significa que o conjunto de instantes de tempo que ocorrem saltos é denso em $[0 ; \infty[$. Esta prova pode ser modificada para que a medida de Lévy atribui massa (átomos) a certos conjuntos finitos.

Assim, qualquer distribuição infinitamente divisível é a distribuição de um processo de Lévy no instante $t=1$. A fórmula de Lévy-Khinchin também fornece uma representação geral da função característica de qualquer distribuição infinitamente divisível:

\section{Teorema 2.2 Função Característica das distribuição infinitamente divisíveis Seja} F uma distribuição infinitamente divisível em $\mathbb{R}^{d}$. A sua função característica pode ser representada como:

$$
\begin{aligned}
\Phi_{F} & =\exp \{\psi(z)\}, z \in \mathbb{R}^{d} \\
\psi(z) & =-\frac{1}{2} z . \Sigma z+i \gamma . z+\int_{\mathbb{R}^{d}}\left(\exp \{i z . x\}-1-i z . x 1_{\{|x| \leq 1\}}\right) \nu(d x),
\end{aligned}
$$

sendo que $\Sigma$ é uma matriz positiva definida simétrica de dimensão $d \times d, \gamma \in \mathbb{R}^{d}$ e $\nu$ uma medida de Radon positiva em $\mathbb{R}^{d} \backslash\{0\}$ sujeita à

$$
\int_{|x| \leq 1}|x|^{2} \nu(d x)<\infty \quad \int_{|x| \geq 1} \nu(d x)<\infty
$$

onde $\nu$ é a medida de Lévy da distribuição $F$.

O Quadro 2.1 relaciona função característica de algumas distribuições infinitamente divisíveis:

\subsection{Trajetórias de um Processo de Lévy}

Utilizando decomposição de Lévy-Itô é possível deduzir algumas propriedades dos caminhos observados de um processo de Lévy por meio das propriedades do seu tripé característico $(\Sigma, \nu, \gamma)$.

Trajetórias Constantes por Partes A Proposição 2.3 prova que quase todas as trajetórias de um processo de Lévy são constantes por partes se e somente se o processo for composto de Poisson. Combinando esta propriedade com a fórmula ex- 
Quadro 2.1. Exemplos de Distribuições e Funções Características

\begin{tabular}{|l|c|c|}
\hline Distribuição & Densidade (f.d.p.) $\mu(d x) / d x$ & Função Característica $\Phi(z)$ \\
\hline Exponencial $(\alpha>0)$ & $\alpha \exp (-\alpha x) 1_{x \geq 0}$ & $\frac{\alpha}{\alpha-i z}$ \\
Laplace $(\alpha>0)$ & $\frac{\alpha}{2} \exp (-\alpha|x|)$ & $\frac{\alpha}{\alpha^{2}+|z|^{2}}$ \\
Gama $(\alpha>0)$ & $\frac{\lambda^{\alpha}}{\Gamma(\alpha)} x^{\alpha-1} e^{-\lambda x} 1_{x \geq 0}$ & $\frac{1}{\left(1-i \lambda^{-1} z\right)^{\alpha}}$ \\
Gaussiana $(\sigma>0)$ & $\frac{1}{\sqrt{2 \pi \sigma}} \exp \left[-\frac{1}{2}\left(\frac{x-\gamma}{\sigma}\right)^{2}\right]$ & $\exp \left(-\frac{\sigma^{2} z^{2}}{2}+i \gamma z\right)$ \\
Cauchy $(\alpha>0)$ & $\frac{\alpha}{\pi\left[(x-\gamma)^{2}+\alpha^{2}\right]}$ & $\exp (-\alpha|z|+i \gamma z)$ \\
Simétrica $\alpha$-estável $(\alpha, b>0)$ & Não conhecida & $\exp \left(-\alpha|z|^{\beta}\right)$ \\
t de Student $(\nu>1)$ & $\frac{\Gamma[(\nu+1) / 2]}{\sqrt{\nu \pi} \Gamma(\nu / 2)}\left(1+\frac{x^{2}}{\nu}\right)$ & Não conhecida \\
\hline
\end{tabular}

ponencial de um processo composto de Poisson, equação (2.4), obtém-se o seguinte critério:

Proposição 2.8 Um processo de Lévy tem trajetórias constantes por partes se e somente se seu tripé característico satisfaz as seguintes condições:

$$
\begin{aligned}
& \Sigma=0, \\
& \int_{\mathbb{R}^{d}} \nu(d x)<\infty, \\
& \gamma=\int_{|x| \leq 1} x \nu(d x),
\end{aligned}
$$

ou, de maneira equivalente, se o seu expoente característico apresentar a forma:

$$
\psi(z)=\int_{-\infty}^{\infty}(\exp \{i z x\}-1) \nu(d x), \quad \nu(\mathbb{R})<\infty .
$$

Processo de Lévy com Variações Finitas Cabe lembrar que a variação de uma função $f:[a ; b] \longrightarrow \mathbb{R}^{d}$ é dada por:

$$
V T(f)=\sup \sum_{i=1}^{n}\left|f\left(t_{i}\right)-f\left(t_{i-1}\right)\right|,
$$

sendo que o supremo é obtido por meio de todas as partições finitas $a=t_{0}<t_{1}<$ $\cdots<t_{n-1}<t_{n}=b$ dentro do intervalo $[a ; b]$. Em particular, toda função unidimen- 
sional crescente ou descrescente é de variação finita se a função de variação finita puder ser escrita como a diferença de duas funções crescentes. Um processo de Lévy é de variação finita se suas trajetórias são função de variação finita com probabilidade 1 .

Proposição 2.9 Variação Finita de um Processo de Lévy Um processo de Lévy é de variação finita se e somente se o seu tripé característico $(\Sigma, \nu, \gamma)$ satisfaz:

$$
\Sigma=0, \quad \int_{|x| \leq 1}|x| \nu(d x)<\infty .
$$

Prova (parte "se") Sob as condições apresentadas, $X_{t}$ pode ser representado da seguinte maneira:

$$
X_{t}=b t+\int_{|x| \geq 1, s \in[0 ; t]} x J_{X}(d s \times d x)+\lim _{\varepsilon \downarrow 0} \tilde{X}_{t}^{\varepsilon},
$$

sendo que

$$
\tilde{X}_{t}^{\varepsilon}=\int_{\varepsilon \leq|x|<1, s \in[0 ; t]} x J_{X}(d s \times d x) .
$$

Os dois primeiros termos são de variação finita, logo, é necessário apenas verificar o terceiro termo. A sua variação total no intervalo $[0 ; t]$ é

$$
V T\left(\tilde{X}_{t}^{\varepsilon}\right)=\int_{\varepsilon \leq|x| \leq 1, s \in[0 ; t]}|x| J_{X}(d s \times d x) .
$$

Como o integrando à direita é positivo, pelo Teorema de Fubini [Teorema 1.27, Kallenberg (2002), p.14], tem-se que

$$
\begin{aligned}
\mathbb{E}\left[V T\left(\tilde{X}_{t}^{\varepsilon}\right)\right] & =\mathbb{E}\left[\int_{\varepsilon \leq|x| \leq 1, s \in[0 ; t]}|x| J_{X}(d s \times d x)\right] \\
& =\int_{\varepsilon \leq|x| \leq 1, s \in[0 ; t]}|x| \nu(d x) d s \\
& =t \int_{\varepsilon \leq|x| \leq 1}|x| \nu(d x),
\end{aligned}
$$

que converge para um valor finito quando $\varepsilon \longrightarrow 0$. Desta maneira, $\mathbb{E}\left[V T\left(\lim _{\varepsilon \downarrow 0} \tilde{X}_{t}^{\varepsilon}\right)\right]<\infty$, que implica que a variação de $X_{t}$ é quase-certamente finita.

Prova (parte "somente se") Considere a decomposição de Lévy-Itô, equação 2.11, do processo $X_{t}$. Como a variação de qualquer função cadlag é maior ou igual a soma 
dos seus saltos, tem-se que para todo $\varepsilon>0$ :

$$
\begin{aligned}
V T\left(X_{t}\right) & \geq \int_{\varepsilon \leq|x|<1, s \in[0 ; t]}|x| J_{X}(d s \times d x) \\
& =t \int_{\varepsilon \leq|x|<1}|x| \nu(d x)+\int_{\varepsilon \leq|x|<1, s \in[0 ; t]}|x|\left(J_{X}(d s \times d x)-\nu(d x) d s\right) .
\end{aligned}
$$

Utilizando a fórmula exponencial [Proposição 2.6], é possível mostrar que a variância do segundo termo da última linha é igual a $t \int_{\varepsilon \leq|x|<1, s \in[0 ; t]}|x|^{2} \nu(d x)$. Desta maneira, pelo mesmo argumento que foi utilizado para provar a decomposição de Lévy-Itô, o segundo termo converge quase-certamente para um número finito. Assim, se a condição $\int(|x| \wedge 1) \nu(d x)<\infty$ não for satisfeita, o primeiro termo da última linha irá divergir e a variação de $X_{t}$ será infinita. Ao supor que a condição $\int(|x| \wedge 1) \nu(d x)<\infty$ é satisfeita, significa que $X_{t}$ pode ser escrito como:

$$
X_{t}=X_{t}^{c}+\int_{[0 ; t] \times \mathbb{R}^{d}} x J_{X}(d s \times d x)
$$

sendo que o segundo termo é finito em variação. Como as trajetórias de um MB são quase-certamente de variação infinita [Corolário 2.5, Revuz \& Yor (1999), p. 28], se $\Sigma$ for diferente de zero, $X_{t}$ também terá variação infinita. Desta maneira, é necessário que $\Sigma=0$.

A seguinte proposição mostra que no caso de variação finita a decomposição de Lévy-Itô e a representação de Lévy-Khinchin podem ser simplificadas.

\section{Corolário 2.1 Decomposição de Lévy-Itô e Representação de LévyKhinchin} no caso de variação-finita Seja $X=\left\{X_{t}\right\}_{t \geq 0}$ um processo de Lévy de variação finita com tripé de Lévy dado por $(0, \nu, \gamma)$. Assim, X pode ser expresso como a soma dos saltos entre 0 e t e uma t.d. linear:

$$
X_{t}=b t+\int_{[0 ; t] \times \mathbb{R}^{d}} x J_{X}(d s \times d x)=b t+\sum_{s \in[0 ; t]}^{\Delta X_{s} \neq 0} \Delta X_{s}
$$

e a sua função característica pode ser expressa como:

$$
\mathbb{E}\left[\exp \left\{i z . X_{t}\right\}\right]=\exp \left\{t\left[i b . z+\int_{\mathbb{R}^{d}}(\exp \{i z . x\}-1) \nu(d x)\right]\right\},
$$

sendo que $b=\gamma-\int_{|x| \leq 1} x \nu(d x)$.

Cabe notar que, o tripé de Lévy (tripé característico) de $X$ não é dado por $(0, \nu, b)$ e sim por $(0, \nu, \gamma)$. Como já foi observado anteriormente, $\gamma$ não é a quantidade in- 
trínseca e depende da função de truncamento usada na representação de Lévy-Khinchin, enquanto que $b t$ possui uma interpretação intrínseca como a parte contínua (t.d.) de $X$.

Processo Crescente de Lévy (Subordinador) Um processo crescente de Lévy é denominado subordinador porque ele pode ser utilizado para mudar o tempo de outros processos de Lévy. Os subordinadores são operadores importantes na construção de modelos financeiros baseados em processos de Lévy.

Proposição 2.10 Seja $X=\left\{X_{t}\right\}_{t \geq 0}$ um processo de Lévy em $\mathbb{R}$. As seguintes condição são equivalentes:

i. $\quad X_{t} \geq 0$ q.c. para algum $t>0$.

ii. $\quad X_{t} \geq 0$ q.c. para todo $t>0$.

iii. Os caminhos observados de $X$ são quase-certamente não-decrescentes: $t \geq s \Longrightarrow$ $X_{t} \geq X_{s}$ q.c.

iv. $\quad$ O tripé característico de $X$ satisfaz $\Sigma=0, \nu(]-\infty ; 0])=0, \int_{0}^{\infty}(x \wedge 1) \nu(d x)<$ $\infty$ e $b \geq 0$, i.e., $X$ não apresenta nenhum componente de difusão, apenas saltos positivos de variação finita e t.d. positiva.

Prova $\quad i \Longrightarrow i i i$. Para todo $n, X_{t}$ é a soma de $n$ variáveis estocásticas i.i.d. $X_{t / n}, X_{2 t / n}-X_{t / n},, X_{t}-X_{(n-1) t / n}$. Isto significa que todas estas variáveis são não-negativas quase-certamente. Pela mesma lógica, é possível provar que para dois números racionais $p$ e $q$, tal que $0<p<q, X_{q t}-X_{p t} \geq 0$ q.c. Dado que as trajetórias são contínuas a direita, tem-se que $X$ é um processo não-decrescente. De maneira lógica tem-se as implicações $i i i \Longrightarrow i i$ e $i i \Longrightarrow i$.

$i i i \Longrightarrow i v$. Dado que as trajetórias são não-decrescentes, elas são finitas em variação. Desta maneira, $\Sigma=0$ e $\int_{-\infty}^{\infty}(x \wedge 1) \nu(d x)$ estão garantidos. Para que as trajetórias sejam não-descrescentes, é necessário que não exista nenhum salto negativo, $\operatorname{assim} \nu(]-\infty ; 0])=0$. Se a função é não-descrescente, então, mesmo após extrair alguns saltos, ainda se obtém uma função não-descrescente. Ao extrair todos os saltos 
de uma trajetória $X_{t}$, obtém-se uma função determinística $b t$ que, portanto, deve ser não-descrescente. Isto permite concluir que $b \geq 0$.

Um importante exemplo de subordinador pode ser introduzido pela seguinte proposição:

Proposição 2.11 Seja $\left\{X_{t}\right\}_{t \geq 0}$ um processo de Lévy em $\mathbb{R}^{d}$ e $f: \mathbb{R}^{d} \longrightarrow[0 ; \infty[$ uma função positiva, tal que, $f(x)=O\left(|x|^{2}\right)$, quando $x \longrightarrow 0$. Assim, o processo $S=\left\{S_{t}\right\}_{t \geq 0}$ definido como

é um subordinador.

$$
S_{t}=\sum_{s<t}^{\Delta X_{s} \neq 0} f\left(\Delta X_{s}\right)
$$

Prova Inicialmente é necessário provar que a soma dada pela equação (2.19) converge para algo finito. Ao truncar os grandes saltos, é possível supor que para cada $s$, tem-se $\Delta X_{s} \leq \varepsilon$ para algum $\varepsilon>0$ e $f\left(\Delta X_{s}\right) \leq C \Delta X_{s}^{2}$ para algum $C>0$. Assim, tem-se que

$$
\mathbb{E}\left[S_{t}\right]=\int_{[0 ; t] \times \mathbb{R}} f(x) d s \nu(d x)<\infty .
$$

Como todos os termos da equação (2.20) são positivos, isto significa que há convergência quase-certamente finita de $S_{t}$ para todo $t$. O fato de $S$ ter incrementos independentes e estacionários segue diretamente da independência e estacionariedade dos incrementos de $X$. Para provar que o processo $S$ é contínuo em probabilidade (continuidade estocástica), é possível supor que os saltos de $X_{t}$ são limitados e, desta maneira, $\mathbb{E}\left[\left|S_{t}-S_{s}\right|\right] \longrightarrow 0$, a medida que $s \longrightarrow t$. Assim, $S$ é contínuo em probabilidade.

A escolha de $f(x)=x^{2}$ produz a soma quadrática dos saltos

$$
S_{t}=\sum_{s \leq t}^{\Delta X_{s} \neq 0}\left|\Delta X_{s}\right|^{2} .
$$

Este processo, que pela Proposição 2.11, é um subordinador e, pode ser representado por $[X, X]^{d}$ e denominado "variação quadrática descontínua"do processo $X$.

Observação Existem processos de Lévy sem o componente de difusão, que não apresentam saltos negativos, mas que satisfazem a condição $\int_{0}^{1}|x| \nu(d x)=\infty$. A 
Proposição 2.10, garante que estes processos não podem apresentar trajetórias crescentes, independente do coeficiente de tendência determinística. A explicação para este comportamento é que nesta situação o processo não é igual a soma dos seus saltos, porque os saltos devem ser compensados. O termo de compensação é somado por uma tendência "infinitamente forte "de saltos negativos que não pode ser modificada pela tendência positiva do processo.

\subsection{Propriedades Distribucionais}

Se $X=\left\{X_{t}\right\}_{t \geq 0}$ é um processo de Lévy, então, para qualquer $t>0$, a distribuição de $X_{t}$ é infinitamente divisível e a sua função característica apresenta a forma dada pela equação (2.15). Entretanto, nem sempre $X_{t}$ apresentará uma densidade. $\mathrm{Na}$ verdade, se $X_{t}$ for um processo composto de Poisson, tem-se que

$$
\mathbb{P}\left[X_{t}=0\right]=\exp (-\lambda t)>0 .
$$

Assim, a probabilidade da distribuição possui massa zero (ausência de átomo) para todo $t$. Caso $X$ não seja um processo composto de Poisson, então, $X_{t}$ possui densidade contínua. Esta propriedade permite estabelecer a seguinte proposição para $d=1$ [Orey (1968); Proposição 28.3, Sato (1999), p.190]:

Proposição 2.12 Existência de uma Densidade Suave Seja $X=\left\{X_{t}\right\}_{t \geq 0}$ um processo de Lévy de valores reais com tripé de Lévy $\left(\sigma^{2}, \nu, \gamma\right)$.

1. Se $\sigma>0$ ou $\nu(\mathbb{R})=\infty$, então, $X_{t}$ possui densidade contínua $p_{t}(\cdot)$ em $\mathbb{R}$.

2. Se a medida de Lévy $\nu$ satisfaz

$$
\exists \beta \in] 0 ; 2], \liminf _{\epsilon \downarrow 0} \epsilon^{-\beta} \int_{-\epsilon}^{\epsilon}|x|^{2} \nu(d x)>0,
$$

então, para cada $t>0, X_{t}$ tem densidade suave $p_{t}(\cdot)$, tal que

$$
p_{t}(\cdot) \in \mathcal{C}^{\infty}(\mathbb{R}), \forall n \geq 1, \frac{\partial^{n} p_{t}}{\partial x^{n}}(t, x) \underset{|x| \rightarrow \infty}{\longrightarrow} 0 .
$$

Relação entre a Densidade de Probabilidade e a Densidade de Lévy No caso de um processo composto de Poisson existe uma relação simples entre a dis- 
tribuição de probabilidade no instante de tempo $t$ e a distribuição do tamanho do salto em relação a medida de Lévy. Seja $X=\left\{X_{t}\right\}_{t \geq 0}$ um processo composto de Poisson com intensidade $\lambda$, distribuição do tamanho do salto $f$ e $X=\left\{N_{t}\right\}_{t \geq 0}$ o número de saltos de $X$ em $[0 ; t]$. Assim,

$$
\begin{aligned}
\mathbb{P}\left[X_{t} \in A\right] & =\sum_{n=0}^{\infty}\left(\mathbb{P}\left[X_{t} \in A \mid N_{t}=n\right] \times \mathbb{P}\left[N_{t}=n\right]\right) \\
& =\sum_{n=0}^{\infty}\left(\mathbb{P}\left[X_{t} \in A \mid N_{t}=n\right] \frac{\exp \{-\lambda t\}(\lambda t)^{n}}{n !}\right) \\
& =\exp \{-\lambda t\} \delta_{0}+\sum_{n=1}^{\infty}\left(f^{* n}(A) \frac{\exp \{-\lambda t\}(\lambda t)^{n}}{n !}\right),
\end{aligned}
$$

sendo que $f^{* n}$ representa a convolução de ordem $n$-ésima de $f$ e $\delta_{0}$ é a medida de Dirac concentrada em 0. Como pode ser observado, esta medida de probabilidade não possui densidade porque $\mathbb{P}\left[X_{t}=0\right]>0$. Entretanto, se a distribuição do tamanho do salto possui densidade em relação a medida de Lebesgue, então, a distribuição de $X_{t}$ é absolutamente contínua em qualquer instante de tempo, salto quanto $t=0$. Isto ocorre pois a convolução de distribuições absolutamente contínuas também é absolutamente contínua, i.e., a distribuição de $X_{t}$ pode ser decomposta como

$$
\begin{aligned}
\mathbb{P}\left[X_{t} \in A\right] & =\exp \{-\lambda t\} 1_{\{0 \in A\}}+\int_{A} p_{t}^{a c}(x) d x \\
p_{t}^{a c}(x) & =\sum_{n=1}^{\infty} f^{* n}(x) \frac{\exp \{-\lambda t\}(\lambda t)^{n}}{n !}, \forall x \neq 0,
\end{aligned}
$$

sendo que a densidade do tamanho do salto é representada por $f(x)$. $p_{t}^{a c}$ é a densidade condicionada ao fato do processo ter apresentado pelo menos um salto. Isto implica na seguinte relação assintótica:

$$
\lim _{t \downarrow 0} \frac{1}{t} p_{t}^{a c}(x)=\lambda f(x)=\nu(x), \forall x \neq 0,
$$

sendo que $\nu(x)$ é a densidade de Lévy. Isto significa que a densidade de Lévy descreve o comportamento da densidade de probabilidade para pequenas variações no tempo $(d t)$. Esta relação também permite obter a esperança de funções de $X_{t}$ para pequenas variações no tempo, i.e., para qualquer função mensurável limitada $f$, sendo que $f(0)=0$,

$$
\lim _{t \downarrow 0} \frac{1}{t} \mathbb{E}\left[f\left(X_{t}\right)\right]=\lim _{t \downarrow 0} \frac{1}{t} \int_{\mathbb{R}^{d}} f(x) p_{t}(d x)=\int_{\mathbb{R}^{d}} f(x) \nu(d x) .
$$


Em uma situação de atividade infinita, o resultado assintótico apresentado ocorre apenas para funções mensuráveis $f$ que se extinguem na vizinhança do zero [Corolário 8.9, Sato (1999), p.45].

Momentos e Cumulantes O comportamento da cauda da distribuição de um processo de Lévy e seus respectivos momentos são determinados pela medida de Lévy, como é apresentado na proposição a seguir [Teorema 25.3, Sato (1999), p.159]:

\section{Proposição 2.13 Momentos e Cumulantes de um processo de Lévy Seja $X=$} $\left\{X_{t}\right\}_{t \geq 0}$ um processo de Lévy em $\mathbb{R}$ com tripé característico $(\Sigma, \nu, \gamma)$. O n-ésimo momento absoluto de $X_{t}$, i.e. $\mathbb{E}\left[\left|X_{t}^{n}\right|\right]$, é finito para um certo $t$, ou, de maneira equivalente, para cada $t>0$, se e somente se $\int_{|x| \geq 1}|x|^{n} \nu(d x)<\infty$. Neste caso os momentos de $X_{t}$ podem ser calculados por meio da diferenciação da função característica. Em particular, os cumulantes [Subseção C.5] de $X_{t}$ são, de certa maneira, simples:

$$
\begin{aligned}
\mathbb{E}\left[X_{t}\right] & =t\left(\gamma+\int_{|x| \geq 1} x \nu(d x)\right), \\
\kappa_{2}\left(X_{t}\right) & =\mathbb{V}\left[X_{t}\right]=t\left(\Sigma+\int_{-\infty}^{\infty} x^{2} \nu(d x)\right), \\
\kappa_{n}\left(X_{t}\right) & =t \int_{-\infty}^{\infty} x^{n} \nu(d x) \quad \text { para } n \geq 3 .
\end{aligned}
$$

Isto garante que todas as distribuições infinitamente divisíveis sejam leptocúrticas, pois $\kappa_{4}\left(X_{t}\right)>0$. Adicionalmente, os cumulantes da distribuição de $X_{t}$ aumentam linearmente em $t$. Em particular, a curtose e assimetria de $X_{\Delta}$, ou $X_{t+\Delta}-X_{t}$, são dados por

$$
\alpha_{3}\left(X_{\Delta}\right)=\frac{\kappa_{3}\left(X_{\Delta}\right)}{\kappa_{2}^{3 / 2}\left(X_{\Delta}\right)}=\frac{\alpha_{3}\left(X_{1}\right)}{\sqrt{\Delta}} \quad \alpha_{4}(X)=\frac{\kappa_{4}\left(X_{\Delta}\right)}{\kappa_{2}^{2}\left(X_{\Delta}\right)}=\frac{\alpha_{4}\left(X_{1}\right)}{\Delta} .
$$

Desta maneira, os incrementos de um processo de Lévy, ou de forma equivalente, de todas as distribuições infinitamente divisíveis com tripé característico $(\Sigma, \nu, \gamma)$ são leptocúrticas e, a assimetria e curtose decaem com o aumento da escala de tempo que os incrementos são calculados, i.e., a assimetria decai a uma taxa de $\Delta^{-1 / 2}$ e a curtose a uma taxa de $\Delta$.

Proposição 2.14 Momentos Exponenciais Seja $X=\left\{X_{t}\right\}_{t \geq 0}$ um processo de Lévy em $\mathbb{R}$ com tripé característico $(\Sigma, \nu, \gamma)$ e seja $u \in \mathbb{R}$. O momento exponencial 
$\mathbb{E}\left[\exp \left\{u X_{t}\right\}\right]$ é finito para certo $t$ ou, de forma equivalente, para todo $t>0$, se e somente se $\int_{|x|>1} \exp \{u x\} \nu(d x)<\infty$. Neste caso

$$
\mathbb{E}\left[\exp \left\{u X_{t}\right\}\right]=\exp \{t \psi(-i u)\}
$$

sendo que $\psi$ é o expoente característico de um processo de Lévy [Proposição 2.2].

A prova desta proposição pode ser verificada em Sato (1999) [Teorema 25.17, p.165].

\subsection{Distribuições e Processos Estáveis}

Uma característica interessante do MB é a propriedade de auto-similariedade, i.e., se $W$ é um processo de Wiener em $\mathbb{R}$, então,

$$
\forall a>0,\left\{\frac{W_{a t}}{\sqrt{a}}\right\}_{t \geq 0} \stackrel{d}{=}\left\{W_{t}\right\}_{t \geq 0} .
$$

Ao considerar um MB com t.d. $\quad B_{t}=W_{t}+\gamma t$, então, a propriedade de auto-similariedade é verificada por meio da seguinte relação:

$$
\forall a>0,\left\{\frac{B_{a t}}{\sqrt{a}}\right\}_{t \geq 0} \stackrel{d}{=}\left\{B_{t}+\sqrt{a} \gamma t\right\}_{t \geq 0} .
$$

Os processos de Lévy, por sua vez, também apresentam a propriedade de auto-similariedade. Assim, um processo de Lévy $X_{t}$ de valores reais é dito auto-similar se

$$
\forall a>0,\left\{\frac{X_{a t}}{b(a)}\right\}_{t \geq 0} \stackrel{d}{=}\left\{X_{t}\right\}_{t \geq 0} .
$$

Como a função característica de $X_{t}$ apresenta a forma $\Phi_{X_{t}}(z)=\exp \{-t \psi(z)\}$, esta propriedade é equivalente à seguinte propriedade da função característica:

$$
\forall a>0, \exists b(a): \Phi_{X_{t}}(z)^{a}=\Phi_{X_{t}}(z b(a)), \forall z .
$$

O Quadro 2.3 relaciona os processos auto-similares [Embrechts \& Maejima (2002), cap.1] com os processos de Lévy. Assim os processos podem ser classificados em três grandes classes: processos de Lévy, processos gaussianos e processos auto-similares, sendo que o único processo conhecido que participa simultâneamente das três classes é o MB.

As distribuições que verificam esta propriedade são denominadas distribuições estritamente estáveis. Mais precisamente, é possível estabelecer a seguinte definição: 


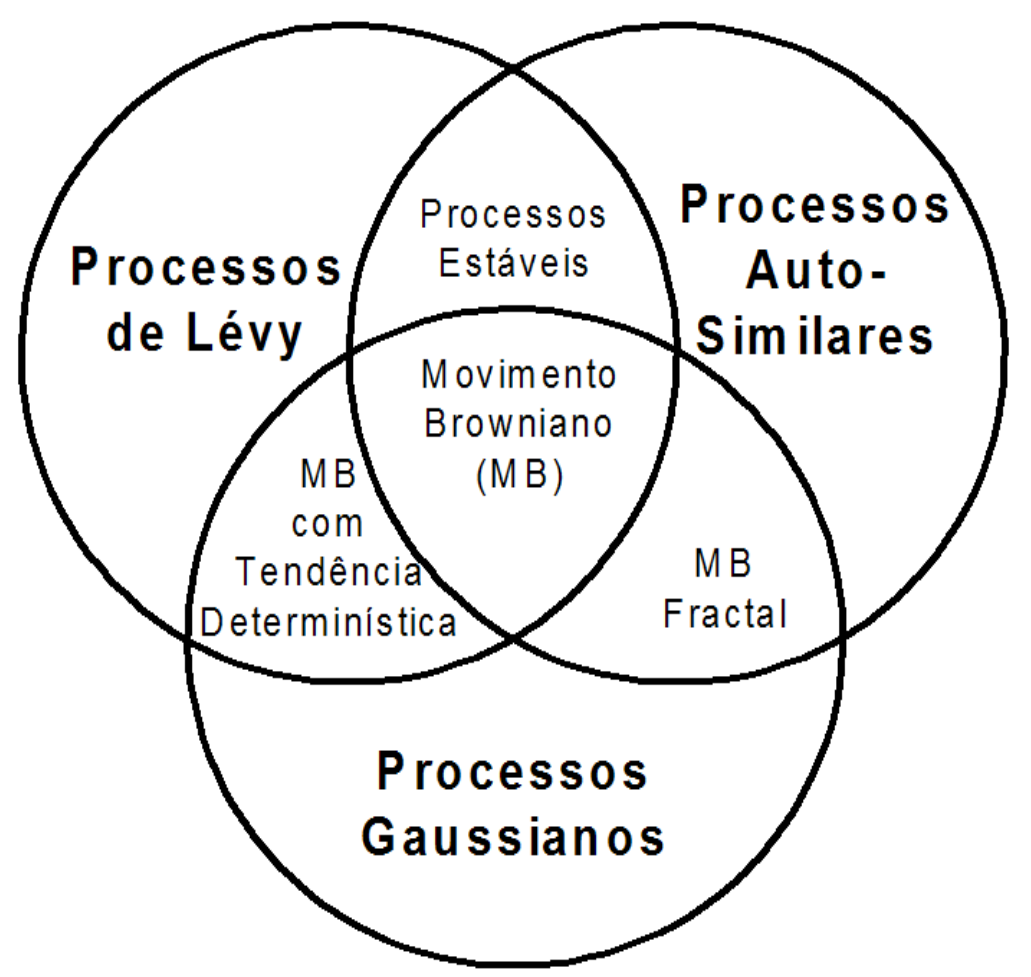

Figura 2.3. A relação entre processos de Lévy, processos auto-similares e processos gaussianos. 
Definição 2.5 Uma v.e. $X \in \mathbb{R}^{d}$ possui uma distribuição estável se para qualquer $a>0$ existir um $b(a)>0$ e um $c(a) \in \mathbb{R}^{d}$, tal que,

$$
\Phi_{X}(z)^{a}=\Phi_{X}(z b(a)) \exp \{i c(a) . z\}, \forall z \in \mathbb{R}^{d} .
$$

A v.e. $X$ possui distribuição estritamente estável se

$$
\Phi_{X}(z)^{a}=\Phi_{X}(z b(a)), \forall z \in \mathbb{R}^{d} .
$$

A denominação estável provém da estabilidade da propriedade de adição, i.e., se $X$ possui distribuição estável e $X^{(1)}, \ldots, X^{(n)}$ são réplicas independentes de $X$, então, existe um escalar positivo $c_{n}$ e um vetor $\mathbf{d}$, tal que

$$
X^{(1)}+\cdots+X^{(n)} \stackrel{d}{=} c_{n} X+\mathbf{d} .
$$

É possível observar esta propriedade se a distribuição $X$ for a distribuição de um processo de Lévy auto-similar em um instante de tempo $t$. Conforme Samorodnitsky \& Taqqu (1994) [Corolário 2.1.3, p.58], para toda distribuição estável existe uma constante $\alpha \in] 0 ; 2]$, tal que $b(a)=a^{1 / \alpha}$ referente à equação (2.28). Esta constante é denominada de indice de estabilidade e, distribuições estáveis com índice de estabilidade $\alpha$ são chamadas de distribuições $\alpha$-estáveis. A única distribuição $\alpha$-estável com $\alpha=2$ é a distribuição gaussiana.

Um processo de Lévy auto-similar possui distribuição estritamente estável para todos os instantes de tempo. Por esta razão, tais processos são denominados de processos de Lévy estritamente estáveis. Um processo de Lévy estritamente $\alpha$-estável satisfaz a seguinte condição:

$$
\forall a>0,\left\{\frac{X_{a t}}{a^{1 / \alpha}}\right\}_{t \geq 0} \stackrel{d}{=}\left\{X_{t}\right\}_{t \geq 0} .
$$

No caso de um processo de Wiener, que possui distribuição gaussiana, i.e., $\alpha=2$, e é um processo estritamente estável, por meio da equação (2.31) é possível obter o resultado apresentado anteriormente. De uma maneira geral, para um processo de Lévy $\alpha$-estável satisfaz a seguinte condição:

$$
\forall a>0,\left\{X_{a t}\right\}_{t \geq 0} \stackrel{d}{=}\left\{a^{1 / \alpha} X_{t}+c t\right\}_{t \geq 0} .
$$

Um processo de Lévy estável define uma família de distribuições estáveis e o contrário também é verdadeiro, i.e., toda distribuição estável é infinitamente divisível 
e, pode ser entendida como uma distribuição estável de um processo de Lévy em um determinado instante de tempo. Conforme Sato (1999) [Teorema 14.3, p.77] e Samorodnitsky \& Taqqu (1994) [Teorema 2.2.1, p.65], este resultado determina a forma do tripé característico de todas os processos de Lévy estáveis e suas distribuições estáveis:

\section{Proposição 2.15 Distribuições Estáveis e Processos de Lévy}

1. Uma distribuição em $\mathbb{R}^{d}$ é $\alpha$-estável com $0<\alpha<2$ se e somente se for infinitamente divisivel com tripé característico $(0, \nu, \gamma)$ e existir uma medida finita $\lambda$ em $S$, uma esfera unitária em $\mathbb{R}^{d}$, tal que,

$$
\nu(B)=\int_{S} \lambda(d \xi) \int_{0}^{\infty} 1_{B}(r \xi) \frac{d r}{r^{1+\alpha}} .
$$

2. Uma distribuição em $\mathbb{R}^{d}$ é $\alpha$-estável com $\alpha=2$ se e somente se for gaussiana.

Para variáveis estocásticas estáveis de valores reais e processos de Lévy unidimensionais $(d=1)$ a representacão acima pode ser feita de maneira explicita, i.e., se $X$ é uma v.e. $\alpha$-estável de valores reais com $0<\alpha<2$, então, sua medida de Lévy tem a seguinte forma:

$$
\nu(x)=\frac{A}{x^{\alpha+1}} 1_{\{x>0\}}+\frac{B}{|x|^{a+1}} 1_{\{x<0\}}
$$

para certas constantes positivas $A$ e $B$. A função característica no instante de tempo $t=1$ de uma v.e. estável de valores reais $X$ possui a forma:

$$
\left\{\begin{array}{cc}
\Phi_{X}(z)=\exp \left\{-\sigma^{\alpha}|z|^{\alpha}\left(1-i \beta \operatorname{sgn}(z) \tan \left(\frac{\pi \alpha}{2}\right)+i \mu z\right)\right\} & \text { se } \alpha \neq 1 \\
\Phi_{X}(z)=\exp \left\{-\sigma^{\alpha}|z|^{\alpha}\left(1-i \beta \frac{2}{\pi} \operatorname{sgn}(z) \ln (|z|)+i \mu z\right)\right\} & \text { se } \alpha=1
\end{array}\right.
$$

\footnotetext{
6 Vôos de Lévy (Lévy flights) Processos de Lévy $\alpha$-estáveis são também conhecidos na literatura da física sob o nome de vôo de Lévy ou difusões anômalas e, são utilizados de maneira extensiva para modelar fenômenos físicos. Vôos de Lévy são processos de Lévy com variância infinita e possuem invariância de escala e propriedades de auto-similariedades. Alguns utilizam a terminologia indevidamente para todos os processos de Lévy, sugerindo de maneira equivocada que todos os processos de Lévy possuem variância infinita. Entretanto, os processos de Lévy são bem mais genéricos que apenas os vôos de Lévy, e não compartilham a maioria das propriedades (com exceção da independência e estacionariedade dos incrementos). Adicionalmente, a literatura física utiliza o termo distribuições de Lévy para todas as distribuições $\alpha$-estáveis não-gaussianas. E por último, para tornar as coisas mais confusas ainda, alguns autores na literatura matemática denominam as distribuições $\alpha$-estáveis $\operatorname{com} \alpha=1 / 2$ de distribuição de Lévy.
} 
sendo que $\alpha \in] 0 ; 2], \sigma \geq 0, \beta \in[-1 ; 1]$ e $\mu \in \mathbb{R}$. Uma distribuição estável em $\mathbb{R}$ nesta parametrização é representada por $S_{\alpha}(\sigma, \beta, \mu)$. Nesta representação, $\sigma$ é o parâmetro de escala (cabe observar que $\sigma$ não tem nada a ver com o parâmetro $\sigma$ de uma distribuição gaussiana se $\alpha<2$ ), $\mu$ é o parâmetro de deslocamento (quando $\alpha \neq 1$ isto não é verdade [Samorodnitsky \& Taqqu (1994), Obs.5, p.8]), $\alpha$ determina a forma da distribuição e $\beta$ a assimetria. Quando $\beta=0$ e $\mu=0, X$ é conhecida por ter uma distribuição simétrica estável e, sua função característica é dada por

$$
\Phi_{X}(z)=\exp \left(-\sigma^{\alpha}|z|^{\alpha}\right) .
$$

A forma explícita da medida de Lévy, equação (2.33), mostra que distribuições $\alpha$ estáveis em $\mathbb{R}$ nunca admitem um segundo momento, elas admitem apenas o primeiro momento, com exceção dos seguintes três casos (e do caso adicional de uma variável determinística):

1. A distribuição gaussiana $S_{2}(\sigma, 0, \mu)$ com densidade (notar a não padronização da parametrização)

$$
\frac{1}{2 \sigma \sqrt{\pi}} \exp \left\{-\frac{(x-\mu)^{2}}{4 \sigma^{2}}\right\} .
$$

2. A distribuição Cauchy $S_{1}(\sigma, 0, \mu)$ com densidade

$$
\frac{\sigma}{\pi\left[(x-\mu)^{2}+\sigma^{2}\right]} \text {. }
$$

3. A distribuição de Lévy $S_{1 / 2}(\sigma, 1, \mu)$ com densidade

$$
\left(\frac{\sigma}{2 \pi}\right)^{1 / 2} \frac{1}{(x-\mu)^{3 / 2}} \exp \left\{-\frac{\sigma}{2(x-\mu)}\right\} 1_{\{x>\mu\}} \text {. }
$$

Enquanto que as duas primeiras distribuições são simétricas em torno da média, a terceira é concentrada em $] \mu ; \infty$ [. Apesar de existir fórmulas fechadas para densidades de probabilidades apenas para estes três casos, existem algoritimos para simular variáveis estocásticas estáveis em $\mathbb{R}$ para todas as parametrizações.

\subsection{Processos de Lévy e Markov}

Uma importante propriedade dos processos de Lévy é a propriedade de Markov, i.e., a v.e. $X_{t}$ quando condicionada à filtragem $\mathcal{F}_{t}$ do processo até o instante de tempo $t$ 
é independente do seu passado anterior àquele instante de tempo. Em outras palavras, para toda variável estocástica $Y$ dependente de uma filtragem $\mathcal{F}_{t}$ de $X_{t}$, tem-se

$$
\mathbb{E}\left[Y \mid \mathcal{F}_{t}\right]=\mathbb{E}\left[Y \mid X_{t}\right]
$$

O núcleo de transição do processo $X_{t}$ é definida como:

$$
\mathbb{P}_{s, t}(x, B) \triangleq \mathbb{P}\left(X_{t} \in B \mid X_{s}=x\right), \forall B \in \mathcal{B}\left(\mathbb{R}^{d}\right),
$$

sendo que $t \geq s$. Uma definição mais formal de uma kernel de transição pode ser obtida em Sato (1999) [Definição 10.1, p.54]. A propriedade de Markov implica na seguinte relação entre as kernels de transição (que são conhecidas como equações de ChapmanKolmogorov):

$$
\mathbb{P}_{s, u}=\int_{\mathbb{R}^{d}} \mathbb{P}_{s, t}(x, d y) \mathbb{P}_{t, u}(y, B),
$$

sendo que $u \geq t \geq s$. É possível observar por meio da equação (2.38) que a kernel de transição de um processo de Lévy é homogênea no espaço e no tempo, i.e.,

$$
\mathbb{P}_{s, t}(x, B)=\mathbb{P}_{0, t-s}(0, B-x),
$$

sendo que $t-s \geq 0$. Em outras palavras, a kernel de transição para processos homogêneos depende apenas do tamanho do intervalo de tempo $t-s$. Os processos de Lévy são completamente caracterizados por esta condição [Teorema 10.5, Sato (1999), p.57].

Os processos de Lévy, por satisfazerem tanto a propriedade de Markov como a propriedade de homogeneidade, pertencem à classe dos processos fortes de Markov, i.e., para todo $t$, o processo $\left\{X_{t+s}-X_{t}\right\}_{s \geq 0}$ possui a mesma distribuição do processo $\left\{X_{s}\right\}_{s \geq 0}$ é independente de $\left\{X_{u}\right\}_{0 \leq u \leq t}$.

Finalmente, a propriedade forte de Markov permite substituir a variável determinística tempo $t$ por qualquer tempo estocástico que seja $\mathcal{F}_{t}$-adaptado (não-antecipável), i.e., se $\tau$ é um tempo estocástico $\mathcal{F}_{t}$-adaptado, então, o processo $\left\{Y_{t}\right\}_{t \geq 0}=\left\{X_{t+\tau}-X_{\tau}\right\}_{t \geq 0}$ é novamente um processo de Lévy independente de $\mathcal{F}_{t} \mathrm{e}$ possui a mesma distribição que $\left\{X_{t}\right\}_{t \geq 0}$.

O operador de transição para processos de Markov pode ser definido como:

$$
\mathbb{P}_{t} f(x)=\mathbb{E}\left[f\left(x+X_{t}\right)\right] .
$$

Equações de Chapman-Kolmogorov que apresentam homogeneidade temporal de kernels de transição são denominadas equações da família semi-grupo, que implicam na 
seguinte relação de operadores de transição:

$$
\mathbb{P}_{t} \mathbb{P}_{s}=\mathbb{P}_{t+s}
$$

Seja $\mathcal{C}_{0}$ o conjunto de funções contínuas que se extinguem no infinito, i.e., $\lim _{x \uparrow \infty} g(x)=0$. Assim, para qualquer $t>0, \mathbb{P}_{t} f(x) \in \mathcal{C}_{0} \mathrm{e}$

$$
\forall x, \lim _{t \downarrow 0} \mathbb{P}_{t} f(x)=f(x),
$$

sendo que a convergência ocorre no sentido da norma do supremo em $\mathcal{C}_{0}$, i.e., $\lim _{n \uparrow \infty}\left\|g_{n}-g\right\|=0, g \in \mathcal{C}_{0}$. Esta propriedade é denominada de propriedade de Feller. Um semi-grupo que $\mathbb{P}_{t}$ que satisfaça a propriedade de Feller, equação (2.39), pode ser descrito em função do gerador infinitesimal $L$, que é o operador linear definido como:

$$
L f=\lim _{t \downarrow 0} t^{-1}\left(\mathbb{P}_{t} f-f\right),
$$

sendo que, a convergência ocorre no sentido da norma suprema em $\mathcal{C}_{0}$ e $f$ deve ser contínua à direita. O gerador infinitesimal de um processo de Lévy pode ser expresso em termos do seu tripé característico [Teorema 31.5, Sato (1999), p.208]:

Proposição 2.16 Gerador Infinitesimal de um Processo de Lévy Seja $X=$ $\left\{X_{t}\right\}_{t \geq 0}$ um processo de Lévy em $\mathbb{R}^{d}$ com tripé característico $(\Sigma, \nu, \gamma)$. O gerador infinitesimal de $X$ é definido para qualquer $f \in \mathcal{C}_{0}^{2}\left(\mathbb{R}^{d}\right)$ como:

$$
\begin{aligned}
(\mathcal{A} f)(x)= & \frac{1}{2} \sum_{j, k=1}^{d} \Sigma_{j k} \frac{\partial^{2} f}{\partial x_{j} \partial x_{k}}(x)+\sum_{j=1}^{d} \gamma_{j} \frac{\partial f}{\partial x_{j}}(x) \\
& +\int_{\mathbb{R}^{d}}\left(f(x+y)-f(x)-\sum_{j=1}^{d} y_{j} \frac{\partial f}{\partial x_{j}}(x) 1_{\{|y| \leq 1\}}\right) \nu(d y)(2.4
\end{aligned}
$$

sendo que $\mathcal{C}_{0}^{2}\left(\mathbb{R}^{d}\right)$ é o conjunto de funções duplamente diferenciáveis que se extinguem no infinito.

Por meio do gerador infinitesimal de um processo de Lévy, é possível verificar que os processos difusões [Definição 1.11] são casos particulares dos processos de Lévy. O gerador infinitesimal de uma difusão e, conseqüentemente de um MB [equação (1.17)] é produzido apenas pelos dois primeiros termos da equação (2.41). Assim, para se obter uma difusão é necessário que a medida de Lévy $\nu\left(\mathbb{R}^{d}\right)=0$. 


\subsection{Martingales e Processos de Lévy}

O conceito de martingale é crucial para a teoria de probabilidade e para a modelagem em finanças. Martingales distintas podem ser construídas ao utilizar a propriedade de independência dos incrementos dos processos de Lévy.

\section{Proposição 2.17}

Seja $\left\{X_{t}\right\}_{t \geq 0}$ um processo de valores reais com incrementos independentes, então,

1. $\left\{\frac{\exp \left(i u X_{t}\right)}{\mathbb{E}\left[\exp \left(i u X_{t}\right)\right]}\right\}_{t \geq 0}$ é uma martingale para $\forall u \in \mathbb{R} .^{7}$

2. Se para algum $u \in \mathbb{R}, \mathbb{E}\left[\exp \left(u X_{t}\right)\right]<\infty, \forall t \geq 0$, então, $\left\{\frac{\exp \left(u X_{t}\right)}{\mathbb{E}\left[\exp \left(u X_{t}\right)\right]}\right\}_{t \geq 0}$ é uma martingale.

3. Se $\mathbb{E}\left[X_{t}\right]<\infty \forall t \geq 0$, então, $M_{t}=X_{t}-\mathbb{E}\left[X_{t}\right]$ é uma martingale com incrementos independentes.

4. Se $\mathbb{V}\left[X_{t}\right]<\infty \forall t \geq 0$, então, $\left(M_{t}\right)^{2}-\mathbb{E}\left[\left(M_{t}\right)\right]$ é uma martingale, sendo que $M_{t}$ é a martingale definida acima.

Se $\left\{X_{t}\right\}_{t \geq 0}$ é um processo de Lévy, para todos os processos definidos nesta proposição, é suficiente que os momentos correspondentes sejam finitos apenas para um $t$ [Teorema 25.3, Sato (1999), p.159].

Em certas circunstâncias de modelagem em finanças, pode ser interessante verificar se um determinado processo de Lévy ou seu exponencial é, ou não, uma martingale. Segue as condições necessárias e suficientes para verificar tal propriedade:

Proposição 2.18 Seja $\left\{X_{t}\right\}_{t \geq 0}$ um processo de Lévy em $\mathbb{R}$ com tripé característico $(\Sigma, \nu, \gamma)$

1. $\left\{X_{t}\right\}$ é uma martingale se e somente se $\int_{|x| \geq 1}|x| \nu(d x)<\infty e$

$$
\gamma+\int_{|x| \geq 1} x \nu(d x)=0 .
$$

7 Esta transformação de variável é denominada transformação de Esscher. 
2. $\exp \left(X_{t}\right)$ é uma martingale se e somente se $\int_{|x| \geq 1} \exp \{x\} \nu(d x)<\infty$ e

$$
\frac{1}{2} \Sigma+\gamma+\int_{-\infty}^{\infty}\left(\exp \{x\}-1-x 1_{\{|x| \geq 1\}}\right) \nu(d x)=0 .
$$

Esta proposição é uma conseqüência natural da Proposição 2.17 e da representação de Lévy-Khinchin. 


\section{Capítulo 3 \\ Modelagem Unidimensional de Processos \\ Estocásticos de Lévy}

\subsection{Salto-Difusão versus Processo de Lévy de Atividade Infinita}

Modelos financeiros com saltos podem ser classificados em duas categorias. A primeira categoria é a denominada modelos de salto-difusão. Nestes modelos, o preço de ativos financeiros evoluem orientados por processos de difusão, e pontualmente ocorrem saltos em intervalos de tempo estocásticos. Os saltos, neste modelo, representam eventos raros, tais como crises financeiras. Tais evoluções podem ser utilizadas para modelarem o log-retorno de ativos financeiros, sendo que estes seguem um processo de Lévy com componentes contínuo e de saltos. O componente contínuo é MB e o componente de saltos é processo composto de Poisson com uma quantidade finita de saltos em cada intervalo de tempo. Exemplos destes tipos de modelos podem ser encontrados na literatura em Merton (1976), onde os saltos possuem uma distribuição gaussiana e Kou (2002), onde os saltos possuem uma distribuição de Laplace (duplamente exponencial). Adicionalmente, as trajetórias dos log-retornos para estes modelos podem ser simuladas por métodos de Monte Carlo ou Quasi-Monte Carlo, permitindo a precificação de derivativos. Apesar destas conveniências, estes modelos não permitem obter de maneira analítica as funções de densidade de probabilidade, o que dificulta calcular de maneira precisa os momentos da distribuição do p.g.d..

A segunda categoria de modelos é representada pelos modelos que apresentam uma quantidade infinita de saltos em um determinado intervalo de tempo, i.e., os modelos de atividade infinita. Não é necessário introduzir nestes modelos a componente contínua (movimento browniano), pois a dinâmica dos saltos já é rica o suficiente para produzir comportamentos não-previsíveis em intervalos de tempo pequenos. Carr et al. (2002) apresentam argumentos para mostrar que estes modelos descrevem de forma mais realista do processo dos preços para diferentes escalas de tempo. Adi- 
Quadro 3.1. Comparação da modelagem por meio de processos compostos de Poisson versus de atividade infinita.

\begin{tabular}{|l|l|}
\hline Modelos de Salto-Difusão & Modelos de Atividade Infinita \\
\hline $\begin{array}{l}\text { Deve conter o componente } \\
\text { do MB }\end{array}$ & $\begin{array}{l}\text { Não contêm necessariamente o } \\
\text { componente do MB }\end{array}$ \\
\hline Saltos são eventos raros & $\begin{array}{l}\text { O processo move-se essencial- } \\
\text { mente por saltos }\end{array}$ \\
\hline $\begin{array}{l}\text { A distribuição do tamanho } \\
\text { dos saltos é conhecida }\end{array}$ & $\begin{array}{l}\text { A distribuição do tamanho dos } \\
\text { saltos não existe, i.e., ocorrem } \\
\text { infinitos saltos }\end{array}$ \\
\hline $\begin{array}{l}\text { Densidade analítica não } \\
\text { conhecida }\end{array}$ & $\begin{array}{l}\text { Densidades analíticas existem } \\
\text { apenas para alguns casos }\end{array}$ \\
\hline Fácil de simular & $\begin{array}{l}\text { Em alguns casos pode ser re- } \\
\text { presentado por subordinação } \\
\text { do movimento Browniano }\end{array}$ \\
\hline
\end{tabular}

cionalmente, muitos modelos desta categoria podem ser construídos por subordinação do MB, como será mostrado adiante, permitindo um melhor manuseio do modelo em relação aos modelos de salto-difusão. Press (1967) propôs um modelo de atividade infinita composto exclusivamente por saltos. Entretanto, este modelo não descreve de maneira realista a dinâmica dos preços.

O Quadro 3.1 compara as vantagens e desvantagens dos modelos de salto-difusão e atividade infinita. Como os preços são observados em escalas discretas, fica difícil saber se os preços empiricamente são produzidos por um processo discreto ou contínuo [Subseção 3.3.1, Campbell et al. (1997)].

Assim, a questão acaba sendo uma escolha conveniente de modelagem. Existem três maneiras convenientes para definir um processo de Lévy paramétrico.

A primeira abordagem é obter um processo de Lévy ao subordinar um MB com um processo crescente de Lévy independente. Nesta abordagem é possível obter a função característica do processo resultante. Contudo, nem sempre a fórmula analítica para a medida de Lévy é conhecida. Dado que a estrutura do processo condicionado segue uma distribuição gaussiana, é viável simular as trajetórias por esta abordagem. A interpretação do subordinador como o tempo de negócio [Geman et al. (2001)], permite uma interpretação econômica do modelo. Extensões multidimensionais são possíveis, i.e., é possível construir um MB multidimensional e mudar a escala do tempo de todos os componentes pelo mesmo subordinador. Entretanto, ao utilizar este procedimento, 
obtêm-se estruturas de dependências com características que não são economicamente bem justificáveis.

A segunda abordagem é especificar diretamente uma medida de Lévy. Esta abordagem permite visualizar a dinâmica do processo de Lévy pois é conhecida a estrutura dos saltos e, pela representação de Lévy-Khinchin, é conhecida a distribuição do processo a qualquer instante de tempo.

A terceira abordagem é especificar uma densidade infinitamente divisível, para a densidade dos incrementos em uma escala de tempo $\Delta$. Os processos com distribuição hiperbólica generalizada podem ser construídos desta maneira. Nesta abordagem, é possível simular os incrementos na escala de tempo determinada e estimar os parâmetros da distribuição se os dados forem coletados na escala de tempo $\Delta$. Entretanto, de uma maneira geral, a medida de Lévy será desconhecida. Assim, a menos que a distribuição pertença a uma classe que sob convoluções seja paramétrica, não será possível identificar a distribuição em outras escalas de tempo. Em particular, dado uma distribuição infinitamente divisível, pode não ser possível verificar se o processo de Lévy possui, ou não, o componente do $\mathrm{MB}$, ou se possui atividade finita ou infinita.

\subsection{Transformando os Processos de Lévy}

A construção de modelos financeiros pode ser efetuada por meio da transformações de processos de Lévy já existentes. Para construir processos de Lévy por meio de processos de Lévy já existentes, pode-se realizar três tipos de transformações, sob as quais os processos de Lévy são invariantes: transformação linear, subordinação (mudança do tempo de um processo de Lévy por meio de um outro processo crescente de Lévy) e inclinação exponencial da medida de Lévy.

\subsubsection{Transformação Linear}

O seguinte teorema é necessário para caracterizar esta transformação:

Teorema 3.1 Seja $\left\{X_{t}\right\}_{t \geq 0}$ um processo de Lévy em $\mathbb{R}^{d}$ com tripé característico $(\Sigma, \nu, \gamma)$ e $M$ é uma matriz de dimensão $n \times d$. Assim, $Y_{t}=M X_{t}$ é um processo de 
Lévy em $\mathbb{R}^{d}$ com tripé característico $\left(\Sigma_{Y}, \nu_{Y}, \gamma_{Y}\right)$, sendo que

$$
\begin{aligned}
\Sigma_{Y} & =M \Sigma M^{T}, \\
\nu_{Y}(B) & =\nu(\{x: M x \in B\}), \forall B \in \mathcal{B}\left(\mathbb{R}^{n}\right), \\
\gamma_{Y} & =M \gamma+\int_{\mathbb{R}^{d}} y\left(1_{\{|y| \leq 1\}}(y)-1_{S_{1}}(y)\right) \nu_{Y}(d y) .
\end{aligned}
$$

$S_{1}$ é a imagem de $M$ de uma bola unitária em $\mathbb{R}^{d}: S_{1}=\{M x:|x| \leq 1\}$.

Prova É possível observar que $Y=\left\{Y_{t}\right\}_{t \geq 0}$ é um processo de Lévy. Assim, esta prova consiste em provar que a equação (3.2) define uma medida de Lévy e que a integral referente a equação (3.3) é finita e que $\left(\Sigma_{Y}, \nu_{Y}, \gamma_{Y}\right)$ é o tripé característico de $Y$. A medida $\nu_{Y}$ é uma medida positiva em $\mathbb{R}^{d}$. A medida $\nu_{Y}$ satifaz

$$
\int_{\mathbb{R}^{n}}\left(|y|^{2} \wedge 1\right) \nu_{Y}(d y)=\int_{\mathbb{R}^{d}}\left(|M x|^{2} \wedge 1\right) \nu(d x)<\infty,
$$

porque a norma de $M$ é finita. Assim, $\nu_{Y}$ é uma medida de Lévy em $\mathbb{R}^{n}$. Dada a integral apresentada na equação (3.3), é suficiente integrar não em relação à todo $\mathbb{R}^{n}$, mas apenas em relação ao sub-espaço $S \subseteq \mathbb{R}^{n}$ definido como $S=\left\{M x: x \in \mathbb{R}^{d}\right\}$, porque a medida $\nu_{Y}$ está concentrada neste sub-espaço. Desta maneira, é possível multiplicar o integrando por uma função indicadora deste sub-espaço e obter

$$
f(y)=y\left(1_{\{|y| \leq 1\}}(y)-1_{S_{1}}(y)\right) 1_{S}(y) .
$$

É possível mostrar que esta integral é finita, ao verificar que existem duas constantes $0<c<C<\infty$, tal que, $f(y)=0$ para qualquer $|y| \geq C$ e $f(y)=0$ para todo $y$, tal que, $|y| \leq c$.

$C$ pode ser qualquer constante maior que $\max (|M|, 1)$, o que implica em $|y|>$ $\max (|M|, 1) \Longrightarrow|y|>1$. Na verdade, se $|y|>\max (|M|, 1)$, então não é possível encontrar um $x \in \mathbb{R}^{d}$ que satisfaça ao mesmo tempo $M x:|x| \leq 1 \mathrm{e}|y| \leq 1$. Logo, $1_{\{|y| \leq 1\}}(y)$ e $1_{S_{1}}(y)$ são ambos iguais a zero e $f(y)=0$.

Por outro lado, o mapeamento $\tilde{M}: \mathbb{R}^{d} \longrightarrow S$, que associa cada $x \in \mathbb{R}^{d}$ à um $M x$, mapeia conjuntos abertos em conjuntos abertos ${ }^{8}$. Desta maneira, o conjunto $\tilde{S}_{1}=\left\{M x: x \in \mathbb{R}^{d},|x|<1\right\}$ é aberto em $S$. Isto significa que o conjunto $S_{1} \cap$ $\{y \in S:|y|<1\}$ também é um conjunto aberto em $S$. Dado que este conjunto contém

8 Segundo Cont \& Tankov (2004b), cap.4, p.107, o mapeamento representa uma sobrejeção linear de espaços de Banach, sendo possível aplicar o Teorema do Mapeamento Aberto. 
o zero, é possível achar um valor na vizinhança do zero em $S$, tal que $f(y)=0$ para cada $y$ desta vizinhança. Isto significa que existe um $c>0$, tal que, $f(y)=0$ para todo $y$, sendo que $|y| \leq c$. Isto prova que a integral em análise pode ser definida no intervalo $[c ; C]$, o que prova sua finitude.

Por último, para completar a prova, é possível utilizar a representação de LévyKhinchin para o processo $X_{t}$ :

$$
\begin{aligned}
\mathbb{E}[ & \left.\exp \left\{i u \cdot M X_{t}\right\}\right]=\mathbb{E}\left[\exp \left\{i M^{T} u \cdot X_{t}\right\}\right] \\
= & \exp \left\{t \left(-\frac{1}{2} M^{T} u \cdot \Sigma M^{T} u+i \gamma \cdot M^{T} u+\right.\right. \\
& \left.\left.\int_{\mathbb{R}^{d}}\left(\exp \left\{i M^{T} u \cdot x\right\}-1-i M^{T} u \cdot x 1_{\{|x| \leq 1\}}\right) \nu(d x)\right)\right\} \\
= & \exp \left\{t \left(-\frac{1}{2} u \cdot M \Sigma M^{T} u+i M \gamma \cdot u+\right.\right. \\
& \left.\left.\int_{\mathbb{R}^{n}}\left\{\exp (i u \cdot y)-1-i u \cdot y\left(1_{\left\{S_{1} \leq 1\right\}}(y)+1_{\{|y| \leq 1\}}(y)-1_{\{|y| \leq 1\}}(y)\right)\right\} \nu_{Y}(d y)\right)\right\} \\
= & \exp \left\{t \left(-\frac{1}{2} u \cdot \Sigma_{Y} u+i\left\{M \gamma+\int_{\mathbb{R}^{n}} y\left(1_{\{|y| \leq 1\}}(y)-1_{\left\{S_{1} \leq 1\right\}}(y)\right) \nu_{Y}(d y)\right\} \cdot u+\right.\right. \\
& \left.\left.\int_{\mathbb{R}^{n}}\left\{\exp (i u \cdot y)-1-i u \cdot y 1_{\{|y| \leq 1\}}(y)\right\} \nu_{Y}(d y)\right)\right\} \\
= & \exp \left\{t\left(-\frac{1}{2} u \cdot \Sigma_{Y} u+i \gamma_{Y} \cdot u+\int_{\mathbb{R}^{n}}\left\{\exp (i u \cdot y)-1-i u \cdot y 1_{\{|y| \leq 1\}}\right\} \nu_{Y}(d y)\right)\right\} . \boldsymbol{\square}
\end{aligned}
$$

Exemplo 3.1 - Soma de Processos de Lévy Independentes Seja $\left\{X_{t}^{1}, X_{t}^{2}\right\}_{t \geq 0}$ um processo bidimensional de Lévy em $\mathbb{R}^{2}$, com tripé característico $(\Sigma, \nu, \gamma)$, sendo que os tripés característicos dos processos marginais são, respectivamente, $\left(\Sigma_{1}, \nu_{1}, \gamma_{1}\right)$ e $\left(\Sigma_{2}, \nu_{2}, \gamma_{2}\right)$. Ao aplicar o Teorema 3.1 para $M=\left[\begin{array}{l}1 \\ 1\end{array}\right]$, obtém-se que $X_{t}^{1}+X_{t}^{2}$ é um processo de Lévy com tripé característico $(\Sigma, \nu, \gamma)$, sendo que,

$$
\Sigma=M^{T} \Sigma M=\Sigma_{1}+\Sigma_{2},
$$

sendo que

$$
\Sigma=\left[\begin{array}{cc}
\Sigma_{1} & 0 \\
0 & \Sigma_{2}
\end{array}\right]
$$

Dada a independência dos dois processos, pela Proposição 4.3, tem-se que a medida de Lévy é representada por:

$$
\begin{aligned}
\nu(B) & =\nu(\{x: M x \in B\}), \forall B \in \mathcal{B}(\mathbb{R}) \\
& =\nu_{1}(B)+\nu_{2}(B) .
\end{aligned}
$$


É possível achar o supremo do domínio da integral que determina a t.d. do processo ao encontrar o valor da constante $C=\max \{|M|, 1\}=\max \{\sqrt{2}, 1\} \Longleftrightarrow C= \pm \sqrt{2}$. O infimo do domínio da integral, pode se estabelecido pela $c=|x| \leq 1$, o que implica que $c= \pm 1$. Assim, o subconjunto $S \subseteq \mathbb{R}$ para o qual será realizada a integração é $S=[-\sqrt{2} ;-1] \cup[1 ; \sqrt{2}$. Assim, a t.d. do processo será igual a

$$
\gamma=\gamma_{1}+\gamma_{2}-\int_{S} y \nu(d y)
$$

Para um processo de Lévy com variação finita, i.e., $\int_{\mathbb{R}^{d}}|x| \nu(d x)<\infty$, o Teorema 3.1 pode ser simplificado. Seja $\left\{X_{t}\right\}_{t \geq 0}$ um processo de Lévy de variação finita em $\mathbb{R}^{d}$ com função característica

$$
\mathbb{E}\left[\exp \left\{i z \cdot X_{t}\right\}\right]=\exp \left\{i b . z+\int_{\mathbb{R}^{d}}(\exp \{i z \cdot x\}-1) \nu(d x)\right\}
$$

e $M$ uma matriz de dimensão $n \times d$. Assim, $Y_{t}=M X_{t}$ é um processo de Lévy em $\mathbb{R}^{n}$ com medida de Lévy $\nu_{Y}=\nu(\{x: M x \in B\})$ e $b_{Y}=M b$.

\subsubsection{Subordinação}

Seja $S=\left\{S_{t}\right\}_{t \geq 0}$ um subordinador, i.e., um processo que satisfaça uma das condições equivalentes da Proposição 2.10, o que significa que as trajetórias são quasecertamente crescentes. Dado que $S_{t}$ é uma v.e. positiva para todo $t$, é possível descrevêla pela transformada de Laplace ao invés da transformada de Fourier. Seja $(0, \rho, b)$ o tripé característico de $S$, então, a m.g.f. de $S_{t}$ é

$$
\mathbb{E}\left[\exp \left\{u S_{t}\right\}\right]=\exp \{t l(u)\}, \forall u \leq 0,
$$

sendo que $l(u)=b u+\int_{0}^{\infty}(\exp \{u x\}-1) \rho(d x) . \quad l(u)$ é denominada o expoente laplaciano de $S$. Como o processo $S$ é crescente ele pode ser interpretado como uma deformação do tempo e é utilizado para mudar o tempo de outro processo de Lévy como é mostrado pelo seguinte teorema.

Teorema 3.2 Subordinação de um Processo de Lévy Estabeleça um espaço probabilístico $(\Omega, \mathcal{F}, \mathbb{P})$. Seja $X=\left\{X_{t}\right\}_{t \geq 0}$ um processo de Lévy em $\mathbb{R}^{d}$ com expoente característico $\Psi(u)$ e tripé característico $(\Sigma, \nu, \gamma)$ e, $S=\left\{S_{t}\right\}_{t \geq 0}$ um subordinador 
com expoente laplaciano $l(u)$ e tripé $(0, \rho, b)$. Assim, o processo $Y=\left\{Y_{t}\right\}_{t \geq 0}$ definido para cada $\omega \in \Omega$ como $Y(t, \omega)=X(S(t, \omega), \omega)$ é um processo de Lévy. A função característica do processo $Y$ é

$$
\mathbb{E}\left[\exp \left\{i u . Y_{t}\right\}\right]=\exp \{t l(\Psi(u))\},
$$

i.e., o expoente característico de $Y$ é obtido pela composição do expoente laplaciano de $S$ com o exponente característico de X. O tripé $\left(\Sigma^{Y}, \nu^{Y}, \gamma^{Y}\right)$ de $Y$ é dado por:

$$
\begin{aligned}
\Sigma^{Y} & =b \Sigma \\
\nu^{Y}(B) & =b \nu(B)+\int_{0}^{\infty} p_{s}^{X}(B) \rho(d s), \forall B \in \mathcal{B}\left(\mathbb{R}^{d}\right), \\
\gamma^{Y} & =b \gamma+\int_{0}^{\infty} \rho(d s) \int_{|x| \leq 1} x p_{s}^{X}(d x),
\end{aligned}
$$

sendo que $p_{t}^{X}$ é a distribuição de probabilidade de $X_{t}{ }^{9}$

Prova Inicialmente é necessário provar que $Y$ é um processo de Lévy. Seja $\mathcal{F}^{S}=$ $\left\{\mathcal{F}_{t}^{S}\right\}_{t \geq 0}$ a filtragem do processo $S$. Para uma seqüência de tempos $t_{0}<t_{1}<\cdots<t_{n}$ é possível obter, pelas propriedades de independência e estacionariedade dos incrementos dos processos $X$, pela independência dos incrementos de $S$ e, pela representação de Lévy-Khinchin, tem-se que:

$$
\begin{aligned}
\mathbb{E}\left[\prod_{i=1}^{n} \exp \left\{i u_{i} \cdot\left[Y\left(t_{i}\right)-Y\left(t_{i-1}\right)\right]\right\}\right]=\mathbb{E}\left[\prod_{i=1}^{n} \exp \left\{i u_{i} \cdot\left(X\left(S_{t_{i}}\right)-X\left(S_{t_{i-1}}\right)\right)\right\}\right] \\
\quad=\mathbb{E}\left\{\mathbb{E}\left[\prod_{i=1}^{n} \exp \left\{i u_{i} \cdot\left(X\left(S_{t_{i}}\right)-X\left(S_{t_{i-1}}\right)\right)\right\} \mid \mathcal{F}^{S}\right]\right\} \\
=\mathbb{E}\left\{\prod_{i=1}^{n} \mathbb{E}\left[\exp \left\{i u_{i} \cdot\left(X\left(S_{t_{i}}\right)-X\left(S_{t_{i-1}}\right)\right)\right\} \mid \mathcal{F}^{S}\right]\right\} \\
=\mathbb{E}\left\{\prod_{i=1}^{n} \mathbb{E}\left[\exp \left\{i u_{i} \cdot\left(X\left(S_{t_{i}}-S_{t_{i-1}}\right)\right)\right\} \mid \mathcal{F}^{S}\right]\right\} \\
=\mathbb{E}\left\{\prod_{i=1}^{n} \exp \left\{\left(S_{t_{i}}-S_{t_{i-1}}\right) \Psi\left(u_{i}\right)\right\}\right\}=\prod_{i=1}^{n} \mathbb{E}\left[\exp \left\{\left(S_{t_{i}}-S_{t_{i-1}}\right) \Psi\left(u_{i}\right)\right\}\right] \\
\quad=\prod_{i=1}^{n} \mathbb{E}\left[\exp \left\{i u_{i} . X\left(S_{t_{i}}-S_{t_{i-1}}\right)\right\}\right]=\prod_{i=1}^{n} \mathbb{E}\left[\exp \left\{i u_{i} . Y\left(t_{i}-t_{i-1}\right)\right\}\right]
\end{aligned}
$$

9 O processo $Y$ é dito subordinado ao processo $X$. 
Desta maneira, o processo $Y$ tem incrementos independentes e estacionários. Para mostrar que $Y$ é contínuo em probabilidade, observe que cada processo de Lévy é uniformemente contínuo em probabilidade, como conseqüência da propriedade de estacionariedade dos incrementos. Pela desigualdade acima e, para cada $\varepsilon>0$ e $\delta>0$, é possível escrever:

$$
\begin{aligned}
\mathbb{P}\left\{\left|X\left(S_{s}\right)-X\left(S_{t}\right)\right|>\varepsilon\right\} \leq & \mathbb{P}\left\{\left|X\left(S_{s}\right)-X\left(S_{t}\right)\right|>\varepsilon|| S_{s}-S_{t} \mid<\delta\right\} \\
& +\mathbb{P}\left\{\left|S_{s}-S_{t}\right| \geq \delta\right\} .
\end{aligned}
$$

O primeiro termo do lado direito da desigualdade pode ser arbitrariamente pequeno todos os valores de $s$ e $t$, controlando $\delta$. Isto ocorre porque $X$ é uniformemente contínuo em probabilidade. O limite do segundo termo para $s \longrightarrow t$ é sempre zero, porque $S$ é contínuo em probabilidade. Assim,

$$
\lim _{s \rightarrow t} \mathbb{P}\left\{\left|X\left(S_{s}\right)-X\left(S_{t}\right)\right|>\varepsilon\right\} \longrightarrow 0 .
$$

A equação (3.6) é facilmente obtida ao condicionar a função característica em $\mathcal{F}^{S}$ :

$$
\begin{aligned}
\mathbb{E}\left[\exp \left\{i u \cdot X\left(S_{t}\right)\right\}\right] & =\mathbb{E}\left[\mathbb{E}\left[\exp \left\{i u \cdot X\left(S_{t}\right)\right\} \mid \mathcal{F}^{S}\right]\right] \\
& =\mathbb{E}\left[\exp \left\{S_{t} \Psi(u)\right\}\right]=\exp \{t l(\Psi(u))\}
\end{aligned}
$$

Vide Sato (1999) [Teorema 30.1, p.197] para a prova detalhada das expressões da tripé característico de $Y$. Apenas para exemplicar o que ocorre veja o seguinte exemplo. Suponha que $S$ seja um subordinador composto de Poisson com tripé característico $(0, \rho, 0)$. Assim, $Y$ é novamente um processo composto de Poisson com a mesma intensidade, porque ele se move apenas por saltos que ocorrem no mesmos instantes de tempo que $S$. Assim, a sua t.d. e componente gaussiano são iguais a zero. Para calcular a sua medida de salto, suponha que $S$ possua um salto em $t$. Ao condicionar o tamanho do salto do processo $Y$ à $S_{t}-S_{t-}=s, Y$ possui distribuição $p_{s}^{X}$. Ao integrar em relação à medida de salto de $S$, i.e. $\rho$, obtém-se a fórmula (3.7). Finalmente, reescrevendo o tripé característico de $Y$ em relação a função de truncamento $1_{\{|x| \leq 1\}}$, obtém-se a equação (3.8) referente à $\gamma^{Y}$.

Exemplo 3.2 Um subordinador estável é um processo $\alpha$-estável para $\alpha \in] 0 ; 1[$, com medida de Lévy concentrada na metade positiva do eixo e, uma t.d. não-negativa. Este processo é um subordinador, pois atende a última condição da Proposição 2.10. Seja 
$S=\left\{S_{t}\right\}_{t \geq 0}$ um subordinador estável com t.d. igual à zero. O seu expoente laplaciano, para $u>0$, é

$$
l(u)=c_{1} \int_{0}^{\infty} \frac{\exp (u x)}{x^{1+\alpha}} d x=-c_{1} \frac{\Gamma(1-\alpha)}{\alpha}(-u)^{\alpha}
$$

para certa constante $c_{1}>0$. Seja $X=\left\{X_{t}\right\}_{t \geq 0}$ um processo simétrico $\beta$-estável em $\mathbb{R}$ com expoente característico $\Psi(u)=-c_{2}|u|^{\beta}$ para certa constante $\beta>0$. Assim, o processo $Y=\left\{Y_{t}\right\}_{t \geq 0}$, que representa a subordinação do processo $X$ à $S$, possui expoente característico $l(\Psi(u))$ igual a

$$
l(\Psi(u))=-c|u|^{\beta \alpha}
$$

sendo que $c=c_{1} c_{2}^{\alpha} \Gamma(1-\alpha) / \alpha$, i.e., $Y$ é um processo estável simétrico com índice de estabilidade igual a $\beta \alpha$. Em particular, quando $X$ é um MB, o processo subordinado é $2 \alpha$-estável.

\subsubsection{Inclinando e Temperando a Medida de Lévy}

Uma outra maneira para se especificar um processo de Lévy é estabelecendo um tripé de Lévy admissível. Em particular, a medida de Lévy deve atender as seguintes condições:

$$
\int_{|x| \leq 1}|x|^{2} \nu(d x)<\infty \quad \int_{|x| \geq 0} \nu(d x)<\infty .
$$

Qualquer transformação da medida de Lévy, que respeite tais condições de integrabilidade irá produzir um novo processo de Lévy. Exemplos de tais transformações são obtidos ao multiplicar a medida $\nu(\cdot)$ por uma função exponencial. Se existir um $\theta \in \mathbb{R}^{d}$, tal que $\int_{|x| \geq 1} \exp \{\theta . x\} \nu(d x)<\infty$, então, a medida $\tilde{\nu}$, definida como

$$
\tilde{\nu}(d x) \triangleq \exp \{\theta \cdot x\} \nu(d x)
$$

é uma medida de Lévy. Para qualquer processo de Lévy $X=\left\{X_{t}\right\}_{t \geq 0}$ em $\mathbb{R}^{d}$ com tripé característico $(\Sigma, \nu, \gamma)$, o tripé característico $(\Sigma, \tilde{\nu}, \gamma)$ também define um processo de Lévy, denominado transformação de Esscher do processo $X$. A tranformação data pela equação (3.10) é denominada inclinação exponencial da medida de Lévy.

Quando $d=1$, é possível considerar uma versão assimétrica da transformação. Se $\nu$ é uma medida de Lévy em $\mathbb{R}$, então,

$$
\tilde{\nu}(d x)=\nu(d x)\left(1_{\{x \geq 0\}} \exp \left\{-\lambda_{+} x\right\}+1_{\{x<0\}} \exp \left\{-\lambda_{-}|x|\right\}\right),
$$


sendo que $\lambda_{+}$e $\lambda_{-}$são parâmetros positivos. $\tilde{\nu}$ é uma medida de Lévy e define um processo de Lévy no qual os grandes saltos são temperados, i.e., as caudas da medida de Lévy são reduzidas.

\subsection{Modelos do Tipo Salto-Difusão}

Um processo de Lévy do tipo salto-difusão $X=\left\{X_{t}\right\}_{t \geq 0}$ possui a seguinte forma:

$$
X_{t}=\gamma t+\sigma W_{t}+\sum_{i=1}^{N_{t}} Y_{i}
$$

sendo que, $N=\left\{N_{t}\right\}_{t \geq 0}$ é um processo de Poisson que conta a quantidade de saltos de $X$ e $Y_{i}$ é o tamanho do salto (variáveis estocásticas i.i.d.) e, $W=\left\{W_{t}\right\}$ é um processo de Wiener (movimento Browniano padronizado). Para definir completamente um modelo paramétrico, é necessário especificar a distribuição do tamanho dos saltos $\nu_{0}(x)$. A forma como será determinada o comportamento das caudas da medida $\nu_{0}$ depende das hipóteses relacionadas aos comportamento dos eventos extremos, pois o comportamento da cauda da medida de salto influencia de maneira significativa o comportamento da densidade de probabilidade do processo [Proposições 2.13 e 2.14].

No modelo de Merton, vide Merton (1976), o tamanho dos saltos que ocorrem nas trajetórias do processo do log-retorno $X_{t}$ assume distribuição gaussiana, i.e., $Y_{i} \stackrel{d}{=}$ $\mathcal{N}\left(\mu, \delta^{2}\right)$. Isto permite obter a densidade de probabilidade de $X_{t}$ :

$$
\mathbb{P}\left\{X_{t} \in A\right\}=\sum_{k=0}^{\infty}\left(\mathbb{P}\left\{X_{t} \in A \mid N_{t}=k\right\} \mathbb{P}\left\{N_{t}=k\right\}\right),
$$

o que garante que a densidade de probabilidade de $X_{t}$ satifaz

$$
p_{t}(x)=\exp \{-\lambda t\} \sum_{k=0}^{\infty} \frac{(\lambda t)^{k} \exp \left\{-\frac{(x-\gamma t-k \mu)^{2}}{2\left(\sigma^{2} t+k \delta^{2}\right)}\right\}}{k ! \sqrt{2 \pi\left(\sigma^{2} t+k \delta^{2}\right)}} .
$$

No modelo de Kou, vide Kou (2002), a distribuição do tamanho dos saltos é dada por uma distribuição assimétrica de Laplace com densidade da seguinte forma:

$$
\nu_{0}(d x)=\left[p \lambda_{+} \exp \left\{-\lambda_{+} x\right\} 1_{\{x \geq 0\}}+(1-p) \lambda_{-} \exp \left\{-\lambda_{-}|x|\right\} 1_{\{x<0\}}\right]
$$

com parâmetros $\lambda_{+}>0$ e $\lambda_{-}>0$ que controlam o decaimento das caudas da distribuição dos saltos com tamanhos positivos e negativos e, $p \in[0 ; 1]$ representa a pro- 
Quadro 3.2. Dois modelos de salto-difusão: Merton e Kou.

\begin{tabular}{|c|c|c|}
\hline & Modelo de Merton (1976) & Modelos de Kou (2002) \\
\hline Tipo de Modelo & \multicolumn{2}{|c|}{ Saltos compostos de Poisson + movimento Browniano } \\
\hline $\begin{array}{l}\text { Parâmetros } \\
\text { (excluindo a } \\
\text { tendência } \\
\text { determinística) }\end{array}$ & $\begin{array}{l}4 \text { parâmetros: } \sigma \text { (volatilidade } \\
\text { da difusão), } \lambda \text { (intensidade do } \\
\text { salto), } \mu \text { (tamanho médio do } \\
\text { salto) e } \delta \text { (desvio-padrão do } \\
\text { tamanho do salto) }\end{array}$ & $\begin{array}{l}5 \text { parâmetros: } \sigma \text { (volatilidade } \\
\text { da difusão), } \lambda \text { (intensidade do } \\
\text { salto) e } \lambda_{+}, \lambda_{-}, p \text { (parâmetros } \\
\text { da distribuição do tamanho } \\
\text { do salto) }\end{array}$ \\
\hline $\begin{array}{l}\text { Medida de } \\
\text { Lévy } \nu(x)\end{array}$ & $\frac{\lambda}{\delta \sqrt{2 \pi}} \exp \left\{-\frac{(x-\mu)^{2}}{2 \delta^{2}}\right\}$ & $\begin{array}{c}p \lambda \lambda_{+} \exp \left\{-\lambda_{+} x\right\} 1_{\{x>0\}}+ \\
(1-p) \lambda \lambda_{-} \exp \left\{-\lambda_{-}|x|\right\} 1_{\{x<0\}}\end{array}$ \\
\hline $\begin{array}{l}\text { Expoente carac- } \\
\text { terístico } \Psi(u)\end{array}$ & $\begin{array}{c}-\frac{\sigma^{2} u^{2}}{2}+i b u+ \\
\lambda\left(\exp \left\{-\frac{\delta^{2} u^{2}}{2}+i \mu u-1\right\}\right)\end{array}$ & $\begin{array}{c}-\frac{\sigma^{2} u^{2}}{2}+i b u+ \\
i u \lambda\left(\frac{p}{\lambda_{+}-i u}-\frac{1-p}{\lambda_{-}-i u}\right)\end{array}$ \\
\hline $\begin{array}{l}\text { Densidade de } \\
\text { Probabilidade }\end{array}$ & Equação (3.12) & Não existe forma analítica \\
\hline $\begin{array}{c}\text { Cumulantes: } \\
E\left[X_{t}\right] \\
V\left[X_{t}\right] \\
\kappa_{3}\left[X_{t}\right] \\
\kappa_{4}\left[X_{t}\right]\end{array}$ & $\begin{array}{c}t(b+\lambda \mu) \\
t\left(\sigma^{2}+\lambda \delta^{2}+\lambda \mu^{2}\right) \\
t \lambda\left(3 \delta^{2} \mu+\mu^{3}\right) \\
t \lambda\left(3 \delta^{3}+6 \mu^{2} \delta^{2}+\mu^{4}\right)\end{array}$ & $\begin{array}{c}t\left(b+\lambda p / \lambda_{+}-\lambda(1-p) / \lambda_{-}\right) \\
t\left(\sigma^{2}+\lambda p / \lambda_{+}^{2}-\lambda(1-p) / \lambda_{-}^{2}\right) \\
t\left(p / \lambda_{+}^{3}-(1-p) / \lambda_{-}^{3}\right) \\
t\left(p / \lambda_{+}^{4}-(1-p) / \lambda_{-}^{4}\right)\end{array}$ \\
\hline $\begin{array}{l}\text { Comportamento } \\
\text { da cauda da } \\
\text { densidade de } \\
\text { probabilidade }\end{array}$ & $\begin{array}{l}\text { Caudas mais grossas que a } \\
\text { normal mas os momentos ex- } \\
\text { ponenciais são todos finitos. }\end{array}$ & $\begin{array}{l}\text { Caudas semi-grossas (expo- } \\
\text { nenciais): } p(x) \sim \exp \left\{-\lambda_{+} x\right\} \\
\text { quando } x \rightarrow \infty \text { e, quando } \\
x \rightarrow-\infty, p(x) \sim \exp \left\{-\lambda_{-}|x|\right\} .\end{array}$ \\
\hline
\end{tabular}

babilidade de saltos positivos. A distribuição de probabilidade dos log-retornos neste modelo possuem semi-leptocurtose. A vantagem deste modelo, ao comparar com o modelo proposto por Merton, é que dada a propriedade de falta de memória das variáveis estocásticas exponenciais, expressões analíticas para a esperança podem ser obtidam [Kou \& Wang (2004)]. Os principais fatores relacionados aos modelos de Merton e Kou são sumarizados no Quadro 3.2.

\subsection{Processos de Lévy via Subordinação Browniana}

Seja $S=\left\{S_{t}\right\}_{t \geq 0}$ um subordinador com expoente laplaciano $l(u)$ e, $W=\left\{W_{t}\right\}_{t \geq 0}$ um MB independente de $S$. Ao subordinar o movimento browniano 
com t.d. $\mu$ à $S$, obtém-se um novo processo de Lévy $X=\left\{X_{t}\right\}_{t \geq 0}$ com a seguinte forma

$$
X_{t}=\sigma W\left(S_{t}\right)+\mu S_{t} .
$$

Este processo é um movimento browniano se for observado em uma nova escala de tempo, i.e., a escala de tempo estocástica data por $S$. Conforme Geman et al. (2001), esta nova escala de tempo possui a interpretação de tempo de negócio, ou seja, a taxa integrada de informação que chega ao conhecimento dos participantes do mercado. Esta interpretação torna os modelos baseados na subordinação browniana mais fáceis de serem compreendidos que os outros modelos de Lévy. A equação (3.6) garante que $X$ possui expoente característico $\Psi(u)=l\left(-u^{2} \sigma^{2} / 2+i u \mu\right)$. Isto permite calcular os cumulantes de $X_{t}$ por meio dos cumulantes de $B_{t}$. Ao considerar o caso simétrico $\mu=0$, tem-se que $X_{t}$ é simétrico e possui esperança e assimetria igual a zero e, é possível calcular a sua variância e excesso de curtose:

$$
\begin{aligned}
\mathbb{V}\left[X_{t}\right] & =\sigma^{2} \mathbb{E}\left[S_{t}\right] \\
\alpha_{4}\left[X_{t}\right] & =\frac{3 \mathbb{V}\left[S_{t}\right]}{\mathbb{E}^{2}\left[S_{t}\right]} .
\end{aligned}
$$

Desta maneira, $X_{t}$ é leptocúrtica se o subordinador não for um processo determinístico, i.e., $\mathbb{V}\left[S_{t}\right]=0$.

Apesar da representação via subordinação browniana ser interpretável e permitir calcular os cumulantes, ela impõe algumas limitações na forma da medida de Lévy. O seguinte teorema caracteriza a medida de Lévy dos processos que podem ser representados pela subordinação browniana com tendência determinística.

Teorema 3.3 Seja $\nu$ uma medida de Lévy em $\mathbb{R}$ e $\mu \in \mathbb{R}$. Existe um processo de Lévy $X=\left\{X_{t}\right\}_{t \geq 0}$ com medida de Lévy $\nu$, tal que, $X_{t}=W\left(Z_{t}\right)+\mu Z_{t}$ para certo subordinador $Z=\left\{Z_{t}\right\}_{t \geq 0}$ e certo $M B W=\left\{W_{t}\right\}_{t \geq 0}$ independente de $Z$ se e somente se as seguintes condições forem satisfeitas:

1. $\nu$ é absolutamente contínua em relação à $\mathbb{R} e$, possui densidade $\nu(x)$.

2. $\nu(x) \exp \{-\mu x\}=\nu(-x) \exp \{\mu x\}$ para todo $x$.

3. $\nu(\sqrt{u}) \exp \{-\mu \sqrt{u}\}$ é uma função completamente monotônica ${ }^{10}$ em $] 0 ; \infty[$.

10 Uma função $f:[a ; b] \longrightarrow \mathbb{R}$ é dita completamente monotônica se todas as suas derivadas existirem e 
Este Teorema permite descrever a estrutura dos saltos de um processo que pode ser representado MB de t.d. com tempo modificado (subordinação browniana). Por exemplo, a medida de Lévy de tal processo possui a versão inclinada (simétrica) em $\mathbb{R}$. Dado que a inclinação exponencial afeta essencialmente os grandes saltos, isto significa que os pequenos saltos de tal processo continuarão simétricos.

Seja $\nu$ uma medida de Lévy em $\mathbb{R}^{d}$. Esta medida de Lévy pode ser a medida de um MB (sem t.d.) subordinado se e somente se for simétrica e $\nu(\sqrt{u})$ for uma função estritamente monotônica em $] 0 ; \infty[$. Considere um subordinador com t.d. igual a zero e uma medida de Lévy $\rho$. A equação (3.7) garante que o movimento browniano com t.d. $\mu$ modificado pelo subordinador terá densidade Lévy $\nu(x)$ igual a

$$
\nu(x)=\int_{0}^{\infty} \frac{1}{\sqrt{2 \pi t}} \exp \left\{-\frac{(x-\mu t)^{2}}{2 t}\right\} \rho(d t) .
$$

É possível denotar simbolicamente esta operação por $B S_{\mu}(\rho)=\nu$, sendo que $B S$ é o acrônimo de subordinação browniana. A transformada inversa é representada por $B S_{\mu}^{-1}(\nu)=\rho$. Assim, partindo da equação (3.14) tem-se:

$$
B S_{\mu}^{-1}(\nu)=\exp \left\{\mu^{2} t / 2\right\} B S_{0}^{-1}(v \exp \{-\mu x\}) .
$$

Assim, é possível verificar por meio da representação da medida de Lévy de um movimento browniano com tempo modificado que a inclinação exponencial da medida $\nu$, i.e., $\nu(x) \exp \{-\mu x\}$ é uma modificação simétrica.

Prova do Teorema 3.3 (parte "somente se") A continuidade absoluta da medida $\nu$ em relação à medida $\rho$ decorre naturalmente da equação (3.7), pois a distribuição gaussiana é absolutamente contínua em relação à $\mathbb{R}$. Ao desprezar a constante $(2 \pi)^{-1 / 2}$, a equação (3.14) pode ser reescrita como:

$$
\nu(x) \exp \{-\mu x\}=\int_{0}^{\infty} \exp \left\{-\frac{x^{2}}{2 t}\right\} \exp \left\{-\frac{\mu^{2} t}{2}\right\} t^{-1 / 2} \rho(d t),
$$

o que mostra que $\nu(x) \exp \{-\mu x\}$ deve ser simétrica em $x$. Se efetuarmos a seguinte transformação $u=x^{2} / 2$ e $s=1 / t$ é possível simplificar a notação acima e, desprezando a constante -1 :

$$
\int_{0}^{\infty} \exp \{-u s\} \exp \left\{-\frac{\mu^{2}}{2 s}\right\} s^{-3 / 2} \rho(d s)=\nu(\sqrt{2 u}) \exp \{-\mu \sqrt{2 u}\},
$$

$(-1)^{k} \frac{d^{k} f(u)}{d u^{k}}>0$ para todo $k \geq 1$. 
o que mostra que $\nu(\sqrt{2 u}) \exp \{-\mu \sqrt{2 u}\}$ é a transformada de Laplace de uma medida positiva e, portanto, pelo Teorema de Bernstein [Feller (1971), v.2, p.439] é uma função completamente monotônica.

Prova do Teorema 3.3 (parte "se") Pela continuidade absoluta de $\nu$ e, dado que o Teorema de Bernstein é condição necessária e suficiente para garantir que a medida seja uma função completamente monotônica, é possível provar, pela mesma linha de raciocínio, que existe uma medida $\rho$ em $] 0 ; \infty[$ que garante a existência da equação (3.14). Entretanto, ainda é necessário provar que $\int_{0}^{\infty}(t \wedge 1) \rho(d t)<\infty$. Pode-se supor, sem perder a generalização da prova, que $\mu \geq 0$. A medida de Lévy $\nu$ do processo subordinado satisfaz $\int_{\mathbb{R}}\left(x^{2} \wedge 1\right) \nu(d x)<\infty$. Ao utilizar o Teorema de Fubini, obtémse que:

$$
\int_{0}^{\infty} \rho(d t) t^{-1 / 2} \int_{\mathbb{R}}\left(x^{2} \wedge 1\right) \exp \left\{-\frac{(x-\mu t)^{2}}{2 t}\right\} d x=\int_{0}^{\infty} \rho(d t) g(t)<\infty .
$$

Para completar a prova do teorema, é necessário mostrar que $g(t) \geq c(t \wedge 1)$ para uma certa constante $c>0$. Depois de efetuar a transformação de variável, $x=u \sqrt{t}+\mu t$, tem-se que

$$
\begin{aligned}
g(t)=t^{-1 / 2} & \int_{\mathbb{R}}\left(x^{2} \wedge 1\right) \exp \left\{-\frac{(x-\mu t)^{2}}{2 t}\right\} d x \\
= & \int_{\mathbb{R}}\left((u \sqrt{t}+\mu t)^{2} \wedge 1\right) \exp \left\{-\frac{u^{2}}{2}\right\} d u \\
& \geq \int_{0}^{\infty}\left((u \sqrt{t}+\mu t)^{2} \wedge 1\right) \exp \left\{-\frac{u^{2}}{2}\right\} d u \\
& \geq \int_{0}^{\infty}\left(u^{2} t \wedge 1\right) \exp \left\{-\frac{u^{2}}{2}\right\} d u
\end{aligned}
$$

Se $t \leq 1$, então,

$$
\begin{gathered}
g(t) \geq \int_{0}^{\infty}\left(u^{2} t \wedge 1\right) \exp \left\{-\frac{u^{2}}{2}\right\} d u \geq \int_{0}^{1}\left(u^{2} t \wedge 1\right) \exp \left\{-\frac{u^{2}}{2}\right\} d u \\
=t \int_{0}^{1} u^{2} \exp \left\{-u^{2} / 2\right\} d u=t c_{1},
\end{gathered}
$$

sendo que $c_{1}>0$. Por outro lado, se $t>1$, então,

$$
\begin{gathered}
g(t) \geq \int_{0}^{\infty}\left(u^{2} t \wedge 1\right) \exp \left\{-\frac{u^{2}}{2}\right\} d u \geq \int_{1}^{\infty}\left(u^{2} t \wedge 1\right) \exp \left\{-\frac{u^{2}}{2}\right\} d u \\
=\int_{1}^{\infty} \exp \left\{-\frac{u^{2}}{2}\right\} d u=c_{2}>0
\end{gathered}
$$


Desta maneira, existe uma constante $c>0$ que $g(t) \geq c(t \wedge 1)$, o que prova o resultado desejado.

\subsubsection{Subordinação do Movimento Browniano}

Considere um subordinador estável temperado, i.e., uma versão temperada exponencialmente do subordinador estável [Exemplo 3.2]. Este processo possui três parâmetros e medida de Lévy igual a

$$
\rho(x)=\frac{c \exp \{-\lambda x\}}{x^{\alpha+1}} 1_{\{x>0\}},
$$

sendo que $c$ e $\lambda$ são constantes positivas e $1>\alpha \geq 0$. Para generalizar inclui-se o caso em que $\alpha=0$ (processo gama), apesar de não ser possível obter este processo por um subordinador estável via inclinação exponencial. Um subordinador estável temperado é um processo estável temperado no sentido da equação (3.29). O parâmetro $c$ altera a intensidade dos saltos de todos os tamanhos simultâneamente, em outras palavras, ele muda a escala de tempo do processo $\lambda$ estabelece a taxa de decaimento dos grandes saltos e, $\alpha$ determina a importância relativa dos saltos pequenos durante a trajetória do processo.

Para um processo estável temperado sem t.d. $b=0$, o expoente laplaciano, para $\alpha \neq 0$, é igual a

$$
\begin{aligned}
l(z)= & \gamma z+\int_{0}^{\infty}\left(\exp \{z x\}-1-z x 1_{\{x \leq 1\}}\right) c \exp \{-\lambda x\} x^{-\alpha-1} d x \\
= & \gamma z+c\left(\int_{0}^{\infty} \exp \{(z-\lambda) x\} x^{-\alpha-1} d x-\int_{0}^{\infty} \exp \{-\lambda x\} x^{-\alpha-1} d x\right. \\
& \left.\quad-\int_{x \leq 1} z x \nu(d x)\right) \\
= & \left(\gamma-c \int_{x \leq 1} x \nu(d x)\right) z+c\left(\int_{0}^{\infty} \exp \{-u\} u^{-\alpha-1} d u\right)\left[(\lambda-z)^{\alpha}-\lambda^{\alpha}\right] \\
= & b z+c \Gamma(-\alpha)\left[(\lambda-z)^{\alpha}-\lambda^{\alpha}\right] \\
= & c \Gamma(-\alpha)\left[(\lambda-z)^{\alpha}-\lambda^{\alpha}\right] .
\end{aligned}
$$


Quadro 3.3. Subordinador Gama versus Subordinador Gaussiana Invertida

\begin{tabular}{|l|c|c|}
\hline Subordinador & Processo Gama & Processo Gaussiano Invertido \\
\hline $\begin{array}{l}\text { Densidade } \\
\text { de Lévy } \rho(x)\end{array}$ & $\frac{c \exp \{-\lambda x\}}{c} 1_{\{x>0\}}$ & $\frac{c \exp \{-\lambda x\}}{x^{3 / 2}} 1_{\{x>0\}}$ \\
\hline $\begin{array}{l}\text { Transformada de } \\
\text { Laplace } E\left[\exp \left\{u S_{t}\right\}\right.\end{array}$ & $\left(1-\frac{u}{\lambda}\right)^{-c t}$ & $\exp \{-2 c t \sqrt{\pi}(\sqrt{\lambda-u}-\sqrt{\lambda})\}$ \\
\hline $\begin{array}{l}\text { Densidade de pro- } \\
\text { babilidade } p_{t}(x)\end{array}$ & $\frac{\lambda^{c t}}{\Gamma(c t)} x^{c t-1}$ & $\frac{c t}{x^{3 / 2}} \exp \{2 c t \sqrt{\pi \lambda}\}$ \\
\hline $\exp \{-\lambda x\} 1_{\{x>0\}}$ & $\times \exp \left\{-\lambda x-\pi c^{2} t^{2} / x\right\} 1_{\{x>0\}}$ \\
\hline
\end{tabular}

Para $\alpha=0$, dado que em torno de $\alpha \downarrow 0$ a função $\Gamma[-\alpha]$ comporta-se como $-1 / \alpha$, tem-se que:

$$
\begin{aligned}
\lim _{\alpha \downarrow 0} c \Gamma(-\alpha)\left[(\lambda-z)^{\alpha}-\lambda^{\alpha}\right]=\lim _{\alpha \downarrow 0}(-c) \frac{\alpha[\ln (\lambda-z)-\ln (\lambda)]}{\alpha} \\
=-c[\ln (\lambda-z)-\ln (\lambda)]=-c \ln \left(1-\frac{z}{\lambda}\right) .
\end{aligned}
$$

A densidade de probabilidade de um subordinador estável temperado só é conhecido na sua forma analítica para $\alpha=1 / 2$ (subordinador gaussiano invertido) e $\alpha=0$ (subordinador gama). Estes dois casos são comparados no Quadro 3.3.

O subordinador estável temperado possui a seguinte propriedade de escalonamento. Seja $S=\left\{S_{t}(\alpha, \lambda, c)\right\}_{t \geq 0}$ um subordinador estável temperado com parâmetros $\alpha, \lambda$ e $c$. Assim, para cada $r>0, r S_{t}(\alpha, \lambda, c)$ possui a mesma distribuição que $S_{r^{\alpha} t}(\alpha, \lambda / r, c)$. Devido a esta propriedade de escalonamento e a propriedade de escolamento do MB ( $r W_{t}$ possui a mesma distribuição que $\left.W_{r^{2} t}\right)$, nos modelos subordinados é suficiente considerar apenas os subordinadores estáveis temperados com $\mathbb{E}\left[S_{t}\right]=t$, que formam uma família de processos com apenas dois parâmetros. Para todos os cálculos relacionados a função característica, momentos e cumulantes, é conveniente utilizar a seguinte parametrização, sendo que $\alpha$ é o índice de estabilidade. Nesta nova parametrização $\kappa$ é igual a variância do subordinador no instante de tempo $t=1$, i.e.,

$$
\begin{aligned}
\mathbb{E}\left[S_{t}\right] & =\left.\frac{d}{d z} t c \Gamma(-\alpha)\left[(\lambda-z)^{\alpha}-\lambda^{\alpha}\right]\right|_{z=0} \\
& =-\left.t c \Gamma(-\alpha) \alpha(\lambda-z)^{\alpha-1}\right|_{z=0}=t c \Gamma(1-\alpha) \lambda^{\alpha-1}
\end{aligned}
$$


quando $\mathbb{E}\left[S_{t}\right]=t$, tem-se que

A variância do subordinador é:

$$
c=\frac{\lambda^{1-\alpha}}{\Gamma(1-\alpha)} .
$$

$$
\begin{aligned}
\mathbb{V}\left[S_{1}\right] & =\left.\frac{d^{2}}{d z^{2}} t c \Gamma(-\alpha)\left[(\lambda-z)^{\alpha}-\lambda^{\alpha}\right]\right|_{z=0, t=1} \\
& =\frac{d}{d z}-\left.t c \Gamma(-\alpha) \alpha(\lambda-z)^{\alpha-1}\right|_{z=0, t=1} \\
& =\left.t c \Gamma(-\alpha)(-\alpha)(1-\alpha)(\lambda-z)^{\alpha-2}\right|_{z=0, t=1} \\
& =(1-\alpha) \lambda^{-1}
\end{aligned}
$$

Ao definir o parâmetro $\kappa=\mathbb{V}\left[S_{1}\right]$, tem-se que $\lambda=(1-\alpha) \kappa^{-1}$ e, a medida de Lévy do subordinador pode ser reescrita como:

$$
\rho(x)=\frac{1}{\Gamma(1-\alpha)}\left(\frac{1-\alpha}{\kappa}\right)^{1-\alpha} \frac{\exp \left\{-\frac{(1-\alpha) x}{\kappa}\right\}}{x^{1+\alpha}} .
$$

Como a esperança do subordinador é igual a 1 no instante de tempo $t=1, \kappa$ determina o quão estocástico será a modificação do tempo e, no caso $\kappa=0$, o subordinador será determinística. No caso da variância gama a fórmula pode ser simplificada como

$$
\rho(x)=\frac{1}{\kappa} \frac{\exp \left\{-\frac{x}{\kappa}\right\}}{x},
$$

e no caso do gaussiano invertido,

$$
\rho(x)=\frac{1}{\sqrt{2 \pi \kappa}} \frac{\exp \left\{-\frac{x}{2 \kappa}\right\}}{x^{3 / 2}} .
$$

\subsubsection{Modelos Baseados na Subordinação Browniana}

Ao modificar o tempo de um MB independente (com volatilidade $\sigma$ e t.d. $\theta$ ) por meio de um subordinador estável temperado, é possível obter o processo denominado gaussiano estável temperado ${ }^{11}$. O expoente característico destes processos é igual a

$$
\Psi(u)=\frac{1-\alpha}{\kappa \alpha}\left\{1-\left(1+\frac{\kappa\left(u^{2} \sigma^{2} / 2-i \theta u\right)}{1-\alpha}\right)^{\alpha}\right\}
$$

para o caso geral e,

para o caso da variância gama $(\alpha=0)$.

$$
\Psi(u)=-\frac{1}{\kappa} \ln \left\{1+\frac{u^{2} \sigma^{2} \kappa}{2}-i \theta \kappa u\right\}
$$

11 É comum adicionar a palavra normal ao nome do subordinador para denominar o processo resultante de uma subordinação browniana. 
Conforme Cont \& Tankov (2004b) [Capítulo 4, p.118], a medida de Lévy um um processo gaussiano estável temperado pode ser calculado por meio da equação (3.7)

$$
\begin{aligned}
\nu(x) & =\int_{0}^{\infty} \frac{1}{\sigma t^{1 / 2} \sqrt{2 \pi}} \exp \left\{-\frac{(x-\theta t)^{2}}{2 \sigma^{2} t}\right\}\left(\frac{c \exp \{-\lambda t\}}{t^{\alpha+1}} 1_{\{x>0\}}\right) d t \\
& =\frac{c}{\sigma \sqrt{2 \pi}} \int_{0}^{\infty} \exp \left\{-\frac{(x-\theta t)^{2}}{2 \sigma^{2} t}-\lambda t\right\} \frac{1}{t^{\alpha+3 / 2}} d t \\
& =\frac{2 c}{\sigma \sqrt{2 \pi}}\left(\frac{\sqrt{\theta^{2}+2 \lambda \sigma^{2}}}{|x|}\right)^{\alpha+1 / 2} \exp \left\{\frac{\theta x}{\sigma^{2}}\right\} K_{\alpha+1 / 2}\left(\frac{|x| \sqrt{\theta^{2}+2 \lambda \sigma^{2}}}{\sigma^{2}}\right) \\
& =\frac{C(\alpha, \kappa, \sigma, \theta)}{|x|^{\alpha+1 / 2}} \exp \left\{\frac{\theta x}{\sigma^{2}}\right\} K_{\alpha+1 / 2}\left(\frac{|x| \sqrt{\theta^{2}+\frac{2}{\kappa} \sigma^{2}(1-\alpha)}}{\sigma^{2}}\right),
\end{aligned}
$$

sendo que $C(\alpha, \kappa, \sigma, \theta)=\frac{2}{\Gamma(1-\alpha) \sigma \sqrt{2 \pi}}\left(\frac{1-\alpha}{\kappa}\right)^{1-\alpha}\left(\theta^{2}+\frac{2}{\kappa} \sigma^{2}(1-\alpha)\right)^{\alpha / 2+1 / 4}$ e $K .(\cdot)$ é uma função Bessel modificada do segundo tipo [vide Apêndice E]. De acordo com o Teorema 3.3, esta medida é uma inclinação exponencial de uma medida simétrica. Ao introduzir as taxas de decaimento das caudas $\lambda_{+}=\frac{1}{\sigma^{2}}\left(\sqrt{\theta^{2}+\frac{2}{\kappa} \sigma^{2}(1-\alpha)}-\theta\right) \mathrm{e}$ $\lambda_{-}=\frac{1}{\sigma^{2}}\left(\sqrt{\theta^{2}+\frac{2}{\kappa} \sigma^{2}(1-\alpha)}+\theta\right)$, é possível escrever esta medida da seguinte maneira:

$$
\nu(x)=\frac{C(\alpha, \kappa, \sigma, \theta)}{|x|^{\alpha+1 / 2}} \exp \left\{\frac{x\left(\lambda_{-}-\lambda_{+}\right)}{2}\right\} K_{\alpha+1 / 2}\left(\frac{|x|\left(\lambda_{-}+\lambda_{+}\right)}{2}\right) .
$$

A partir do comportamento assintótico da fórmula (3.28), tem-se que:

$$
\begin{aligned}
\nu(x) & \sim \frac{1}{|x|^{2 \alpha+1}}, \text { para } x \longrightarrow 0, \\
\nu(x) & \sim \frac{1}{|x|^{\alpha+1}} \exp \left\{-\lambda_{+} x\right\}, \text { para } x \longrightarrow+\infty, \\
\nu(x) & \sim \frac{1}{|x|^{\alpha+1}} \exp \left\{-\lambda_{-}|x|\right\}, \text { para } x \longrightarrow-\infty,
\end{aligned}
$$

ou seja, a medida de Lévy possui comportamento quase estável na vizinhança de $x=0$ e, decaimento exponencial, com taxa de decaimento igual $\lambda_{+}$e $\lambda_{-}$nas caudas.

Dado que a densidade de probabilidade do subordinador estável temperado é conhecido na forma analítica para $\alpha=1 / 2$ e $\alpha=0$, os processos subordinados correspondentes possuem propriedades matemáticas desejáveis, sendo possível simular estes processos. O processo denominado variância gama (VG) foi incialmente utilizado por Madan \& Seneta (1990) e Madan et al. (1998) para modelar o log-retorno de ações, enquanto que o processo gaussiano invertivo foi proposto por Barndorff-Nielsen (1997) e 
Quadro 3.4. Dois modelos baseados na subordinação browniana: processo variância gama e processo normal invertido gaussiano.

\begin{tabular}{|c|c|c|}
\hline Nome do Modelo & Variância Gama & Normal Gaussiana Invertida \\
\hline Tipo de Modelo & $\begin{array}{l}\text { Processo de variação finita com } \\
\text { atividade infinita mas baixa ativi- } \\
\text { dade relativa dos saltos pequenos }\end{array}$ & $\begin{array}{l}\text { Processo de variação infinita } \\
\text { com comportamento estável } \\
(\alpha=1) \text { dos saltos pequenos }\end{array}$ \\
\hline $\begin{array}{l}\text { Parâmetros ex- } \\
\text { cluindo a t.d. }\end{array}$ & \multicolumn{2}{|c|}{$\begin{array}{l}3 \text { parâmetros: } \sigma \text { e } \theta \text { (volatilidade e tendência d.t. do MB) e } \kappa \\
\text { variância do subordinador. }\end{array}$} \\
\hline $\begin{array}{l}\text { Medida de } \\
\text { Lévy } \nu(x)\end{array}$ & $\begin{array}{l}\frac{1}{\kappa|x|} \exp \{A x-B|x|\} \text { com } \\
A=\frac{\theta}{\sigma^{2}} \text { e } B=\frac{\sqrt{\theta^{2}+2 \sigma^{2} / \kappa}}{\sigma^{2}}\end{array}$ & $\begin{array}{c}\frac{C}{|x|} \exp \{A x\} K_{1}(B|x|) \text { com } \\
A=\frac{\theta}{\sigma^{2}}, B=\frac{\sqrt{\theta^{2}+2 \sigma^{2} / \kappa}}{\sigma^{2}} \mathrm{e} \\
C=\frac{\sqrt{\theta^{2}+2 \sigma^{2} / \kappa}}{2 \pi \sigma \sqrt{\kappa}}\end{array}$ \\
\hline $\begin{array}{l}\text { Expoente carac- } \\
\text { rístico } \Psi(u)\end{array}$ & $-\frac{1}{\kappa} \ln \left(1+\frac{u^{2} \sigma^{2} \kappa}{2}-i \theta \kappa u\right)$ & $\frac{1}{\kappa}-\frac{1}{\kappa} \sqrt{1+u^{2} \sigma^{2} \kappa-2 i \theta \kappa u}$ \\
\hline $\begin{array}{l}\text { Densidade de pro- } \\
\text { babilidade } p_{t}(x)\end{array}$ & $\begin{array}{c}C|x|^{\frac{t}{\kappa}-\frac{1}{2}} \exp \{A x\} K_{\frac{t}{\kappa}-\frac{1}{2}}(B|x|) \\
\operatorname{com} C=\sqrt{\frac{\sigma^{2} \kappa}{2 \pi}} \frac{\left(\theta^{2} \kappa+2 \sigma^{2}\right)^{\frac{1}{4}-\frac{\theta}{2 \kappa}}}{\Gamma(t / \kappa)}\end{array}$ & $\begin{array}{c}C \exp \{A x\} \frac{K_{1}\left(B \sqrt{x^{2}+t^{2} \sigma^{2} / \kappa}\right)}{\sqrt{x^{2}+t^{2} \sigma^{2} / \kappa}} \\
\operatorname{com} C=\frac{t}{\pi} \exp \left\{\frac{t}{\kappa}\right\} \sqrt{\frac{\theta^{2}}{\kappa \sigma^{2}}+\frac{1}{\kappa^{2}}}\end{array}$ \\
\hline $\begin{array}{r}\text { Cumulantes: } \\
E\left[X_{t}\right] \\
V\left[X_{t}\right] \\
\kappa_{3}\left[X_{t}\right] \\
\kappa_{4}\left[X_{t}\right]\end{array}$ & $\begin{array}{c}\theta t \\
\theta^{2} \kappa t+\sigma^{2} t \\
3 \sigma^{2} \theta \kappa t+2 \theta^{3} \kappa^{2} t \\
3 \sigma^{4} \kappa t+6 \theta^{4} \kappa^{3} t+12 \sigma^{2} \theta^{2} \kappa^{2} t\end{array}$ & $\begin{array}{c}\theta t \\
\theta^{2} \kappa t+\sigma^{2} t \\
3 \sigma^{2} \theta \kappa t+3 \theta^{3} \kappa^{2} t \\
3 \sigma^{4} \kappa t+15 \theta^{4} \kappa^{3} t+18 \sigma^{2} \theta^{2} \kappa^{2}\end{array}$ \\
\hline $\begin{array}{l}\text { Comportamento } \\
\text { da cauda }\end{array}$ & \multicolumn{2}{|c|}{$\begin{array}{l}\text { Medida de Lévy e densidade de probabilidade com caudas exponen- } \\
\text { ciais com taxa de decaimento igual a } \lambda_{+}=B-A \text { e } \lambda_{-}=B+A\end{array}$} \\
\hline
\end{tabular}

Barndorff-Nielsen (1998). As principais propriedades destes dois processos são sumarizadas no Quadro 3.4.

\subsection{Processo Estável Temperado}

O processo estável temperado é obtido ao se multiplicar a medida de Lévy de um processo estável unidimensional por um exponencial descrescente para cada metade do eixo que representa os números $\mathbb{R}$. Após a suavização exponencial, os pequenos saltos mantêm seu comportamento estável, enquanto que, os maiores saltos se tornam maiores (mais violentos). Um processo estável temperado é um processo de Lévy em $\mathbb{R}$ sem o 
componente gaussiano e densidade de Lévy com a seguinte forma:

$$
\nu(x)=\frac{c}{|x|^{1+\alpha}} \exp \left\{-\lambda_{-}|x|\right\} 1_{\{x<0\}}+\frac{c_{+}}{x^{1+\alpha}} \exp \left\{-\lambda_{+} x\right\} 1_{\{x>0\}},
$$

sendo que os parâmetros satisfazem $c_{-}>0, c_{+}>0, \lambda_{-}>0, \lambda_{+}>0$ e $\alpha<2$. Inicialmente, a modelagem baseada em processos estáveis temperados foi introduzida por Konopen (1995).

Diferentemente dos processos estáveis, que são definidos apenas para $\alpha>0$, os processos estáveis temperados não apresentam limite inferior para $\alpha$ e, equação (3.29) produz medidas de Lévy para todo $\alpha<2$. De fato, ao atribuir valores negativos ou mesmo valores diferentes para $\alpha$ quando $x<0$ e $x>0$ pode-se obter processos interessantes para a modelagem. Assim, o termo processos estáveis temperados será utilizado para representar os processos com medida de Lévy dada pela equação (3.29) com $\alpha>0$, pois apenas neste caso os pequenos saltos apresentarão comportamento estável. O termo processos estáveis temperados generalizados séra utilizado aos processos com medida de Lévy dada pela expressão:

$$
\nu(x)=\frac{c}{|x|^{1+\alpha_{-}}} \exp \left\{-\lambda_{-}|x|\right\} 1_{\{x<0\}}+\frac{c_{+}}{x^{1+\alpha_{+}}} \exp \left\{-\lambda_{+} x\right\} 1_{\{x>0\}},
$$

sendo que, $\alpha_{-}<2$ e $\alpha_{+}<2$. Todas as fórmulas que serão apresentadas nesta parte do estudo consideram os processos estáveis temperados generalizados, sendo que os processos estáveis temperados representam apenas um caso particular destes.

A proposição seguinte mostra que os modelos estáveis temperados permitem estruturas mais complexas que os modelos de subordinação do MB.

\section{Proposição 3.1 Representação de um Movimento Browniano com Tempo} Modificado por um Processo Estável Temperado Um processo estável temperado generalizado pode ser representado como a mudança de tempo de um movimento browniano com t.d. se e somente se $c_{-}=c_{+}=$c e $\alpha_{-}=\alpha_{+}=\alpha \geq-1$.

Conforme Abramowitz \& Stegun (1968) [Capítulo 9, p.686], a medida de Lévy do subordinador desta representação pode ser expressa por funções especiais, i.e., a representação é dada por $\mu=\left(\lambda_{-}-\lambda_{+}\right) / 2 \mathrm{e}$

$$
\rho(t)=\frac{c}{t^{\alpha / 2+1}} \exp \left\{\frac{t \mu^{2}}{2}-\frac{t \lambda^{2}}{4}\right\} D_{-\alpha}(\lambda \sqrt{t}),
$$


sendo que $\lambda=\left(\lambda_{-}+\lambda_{+}\right) / 2$, $c$ é uma constante e $D_{-\alpha}(z)$ representa a função do cilindro parabólico de Whittaker [Abramowitz \& Stegun (1968), p.685-700].

A condição imposta sobre os coeficientes significa que os pequenos saltos devem ser simétricos, enquanto que, as taxas de decaimento dos grandes saltos podem ser diferentes. Carr et al. (2003) apresenta a classe de processos estáveis temperados, denominados CGMY (Carr, Geman, Madan e Yor), que podem ser representados por subordinação do MB. A classe de modelos CGMY tem menos flexibilidade que os modelos baseados nos processos estáveis temperados, que possibilitam assimetria nos saltos pequenos. Apesar disto, o impacto da assimetria gerado sobre a precificação de opções é decorrente principalmente da assimetria dos grandes saltos, o que viabiliza a utilização dos modelos CGMY.

Prova da Proposição 3.1 (parte "se ") Dado que a medida de Lévy $\nu(x)$ é dada pela equação (3.30), é possível verificar que a primeira condição (continuidade absoluta) do Teorema 3.3 é satisfeita. A segunda condição pode ser verificada ao inclinar exponencialmente a medida de Lévy $\nu(x)$ por uma modificação simétrica com $\mu=\left(\lambda_{-}-\lambda_{+}\right) / 2$, i.e.,

$$
\tilde{\nu}(x)=\nu(x) \exp \{-\mu x\}=\frac{c_{-} \exp \{-\lambda|x|\}}{|x|^{\alpha_{-}+1}} 1_{\{x<0\}}+\frac{c_{+} \exp \{-\lambda x\}}{x^{\alpha_{+}+1}} 1_{\{x>0\}}
$$

e

$$
\nu(-x) \exp \{\mu x\}=\frac{c_{+} \exp \{-\lambda|x|\}}{|x|^{\alpha_{+}+1}} 1_{\{x<0\}}+\frac{c_{-} \exp \{-\lambda x\}}{x^{\alpha_{-}+1}} 1_{\{x>0\}},
$$

sendo que as duas equações são equivalentes quando $c=c_{-}=c_{+} \mathrm{e}$ $\alpha=\alpha_{-}=\alpha_{+}$. Neste caso, a medida modificada de Lévy pode ser representada por $\tilde{\nu}(x)=c \frac{\exp \{-\lambda|x|\}}{|x|^{\alpha+1}}$. Para terminar a prova é necessário provar a terceira condição, i.e., $\tilde{\nu}(\sqrt{u})=c \frac{\exp \{-\lambda \sqrt{u}\}}{u^{(\alpha+1) / 2}}$ é completamente monotônica em $\left.u \in\right] 0 ; \infty[$ se e somente se $\alpha \geq-1$. Adicionalmente, tem-se que

$$
\frac{d}{d u}\left[u^{-\frac{\alpha+1}{2}}\right]=-\frac{1}{2}(\alpha+1) u^{-\frac{3+\alpha}{2}},
$$

sendo que $\alpha<1$ implica na possibilidade de alternância do sinal da primeira derivada, i.e., $\tilde{\nu}(x)$ não é uma função monotônica. Se $\alpha \geq 1$, é possível verificar que a função $u^{-\frac{\alpha+1}{2}}$ é completamente monotônica. Por outro lado, $\exp \{-\lambda \sqrt{u}\}$ também é comple- 
tamente monotônica pois

$$
(-1)^{n} \frac{d^{n}}{d u^{n}} \exp \{-\lambda \sqrt{u}\}>0 .
$$

Como o produto de duas funções completamente monotônicas produz uma função completamente monotônica tem-se o resultado desejado.

Prova da Proposição 3.1 (parte "somente se") Pelo resultado apresentado na equação (3.15) é possível mostrar que a medida $\tilde{\nu}(x)$ é inversível quando $\mu$ é uma modificação simétrica em $x$, sendo que a medida de Lévy $\rho(t)$ do subordinador é dado pela equação (3.31), onde $c=c_{-}=c_{+}$e $\alpha=\alpha_{-}=\alpha_{+}$

Se forem impostas algumas condições aos parâmetros da medida de Lévy pode-se obter as seguintes proposições:

Proposição 3.2 Um processo estável temperado generalizado possui variação finita se $\alpha_{+}<1$ e $\alpha_{-}<1$.

Prova Para verificar que uma medida de Lévy estável temperada é de variação finita para $\alpha_{-}, \alpha_{+}<1$, pois

$$
\begin{gathered}
\int_{\mathbb{R}}|x| \nu(d x)=c_{-} \int_{\mathbb{R}}|x|^{-\alpha_{-}} 1_{\{x<0\}} \exp \left\{-\lambda_{-}|x|\right\} d x \\
\quad+c_{+} \int_{\mathbb{R}}|x|^{-\alpha_{+}} 1_{\{x>0\}} \exp \left\{-\lambda_{+} x\right\} d x \\
=c_{-} \int_{\mathbb{R}_{+}} x^{-\alpha_{-}} \exp \left\{-\lambda_{-} x\right\} d x \\
\quad+c_{+} \int_{\mathbb{R}_{+}} x^{-\alpha_{+}} \exp \left\{-\lambda_{+} x\right\} d x
\end{gathered}
$$

Aplicando a seguinte transformação $x=u / \lambda_{-,+}$tem-se

$$
\begin{aligned}
& \int_{\mathbb{R}}|x| \nu(d x)= c_{-} \int_{\mathbb{R}_{+}}\left(\frac{u}{\lambda_{-}}\right)^{-\alpha_{-}} \frac{1}{\lambda_{-}} \exp \{-u\} d u \\
&+c_{+} \int_{\mathbb{R}_{+}}\left(\frac{u}{\lambda_{+}}\right)^{-\alpha_{+}} \frac{1}{\lambda_{+}} \exp \{-u\} d u \\
&= c_{-}\left(\lambda_{-}\right)^{\alpha_{-}-1} \int_{\mathbb{R}_{+}} u^{-\alpha_{-}} \exp \{-u\} d u \\
&+c_{+}\left(\lambda_{+}\right)^{\alpha_{+}-1} \int_{\mathbb{R}_{+}} u^{-\alpha_{+}} \exp \{-u\} d u \\
&=c_{-}\left(\lambda_{-}\right)^{\alpha_{-}-1} \Gamma\left[1-\alpha_{-}\right]+c_{+}\left(\lambda_{+}\right)^{\alpha_{+}-1} \Gamma\left[1-\alpha_{+}\right],
\end{aligned}
$$


sendo que $\Gamma[y] \triangleq \int_{0}^{\infty} u^{-y-1} \exp \{-u\} d u$ é uma função gama completa. Quando $y \in\{0,-1,-2, \ldots\}, \Gamma[y]= \pm \infty, \operatorname{logo}, \alpha_{-}, \alpha_{+}<1$ é condição suficiente para $\int_{\mathbb{R}}|x| \nu(d x)<\infty$. Assim, pela Proposição 2.9 que um processo com $\Sigma=0$ e medida de Lévy $\nu(x)$ igual à apresentada pela equação (3.30) com $\alpha_{-}, \alpha_{+}<1$ é um processo de variação finita.

Proposição 3.3 Um processo estável temperado generalizado é um processo composto de Poisson se $\alpha_{+}<0$ e $\alpha_{-}<0$.

Prova Para que seja verificado que o processo de Lévy com uma medida de Lévy estável temperada possui atividade finita. Ao utilizar a mesma transformação de variável aplicada anteriormente é possível observar que:

$$
\int_{\mathbb{R}} \nu(d x)=c_{-}\left(\lambda_{-}\right)^{\alpha_{-}} \Gamma\left[-\alpha_{-}\right]+c_{+}\left(\lambda_{+}\right)^{\alpha_{+}} \Gamma\left[-\alpha_{+}\right] .
$$

Pelas mesmas razões anteriores, é condição suficiente que $\alpha_{-}, \alpha_{+}<0$ para que $\int \nu(d x)<\infty$. Dado que $\gamma=\int_{|x| \leq 1} x \nu(d x)$ em um processo composto de Poisson, tem-se que o expoente característico de um processo temperado estável é igual a:

$$
\begin{aligned}
\psi(z) & =i \gamma z+\int_{\mathbb{R}}\left(\exp \{i z x\}-1-i z x 1_{\{|x| \leq 1\}}\right) \nu(d x) \\
& =i \gamma z-\int_{\mathbb{R}} i z x 1_{\{|x| \leq 1\}} \nu(d x)+\int_{\mathbb{R}}(\exp \{i z x\}-1) \nu(d x) \\
& =i z\left(\gamma-\int_{|x| \leq 1} x \nu(d x)\right)+\int_{\mathbb{R}}(\exp \{i z x\}-1) \nu(d x) \\
& =\int_{\mathbb{R}}(\exp \{i z x\}-1) \nu(d x) .
\end{aligned}
$$

Conforme Proposições 2.8 e 3.2 tem-se que um processo de Lévy com medida de Lévy dada pela equação (3.30) com coeficiente de difusão igual $\Sigma=0$, e $\alpha_{-}, \alpha_{+}<0$ é um processo composto de Poisson.

Proposição 3.4 Um processo estável temperado generalizado é um subordinador se $\alpha_{+}<1, c_{-}=0$ e $\gamma>0$. 
Prova $\operatorname{Se} c_{-}=0$ tem-se que:

$$
\begin{aligned}
\int_{0}^{1} x \nu(d x) & =c_{+}\left(\lambda_{+}\right)^{\alpha_{+}-1}\left(\Gamma\left[1-\alpha_{+}\right]-\Gamma\left[1-\alpha_{+}, 1\right]\right), \\
\int_{1}^{\infty} \nu(d x) & =c_{+}\left(\lambda_{+}\right)^{\alpha_{+}} \Gamma\left[-\alpha_{+}, 1\right],
\end{aligned}
$$

sendo que $\Gamma[y, k] \triangleq \int_{k}^{\infty} u^{-y-1} \exp \{-u\} d u$ é uma função gama incompleta com limite inferior $k$. Dado que $\Gamma\left[-\alpha_{+}, 1\right]<\infty$ para $\forall \alpha_{+} \in \mathbb{R}$, a condição $\int_{0}^{\infty}(x \wedge 1) \nu(d x)<$ $\infty$ é satisfeita quando $\alpha_{+}<1$. Assim, pela Proposição (2.10) o processo de Lévy com medida de Lévy dada pela equação (3.30) é um subordinador se $\Sigma=0, \gamma>0, c_{-}=0$ e $\alpha_{+}<1$.

O caso limite $\alpha_{-}=\alpha_{+}=0$ corresponde a um processo de atividade infinita. Se, adicionalmente, $c_{-}=c_{+}$, então, obtém-se o modelo variância gama.

Se a t.d. for excluída o processo estável temperado generalizado é um modelo paramétrico com seis parâmetros. Por meio da função característica deste processo é possível analisar qual o papel que cada um destes parâmetros desempenha. Ao considerar um processo temperado estável, é possível escrever a representação de LévyKhinchin sem o trucamento dos saltos grandes, i.e.,

$$
\mathbb{E}\left[\exp \left\{i u X_{t}\right\}\right]=\exp \left\{t\left(i u \gamma_{c}+\int_{-\infty}^{\infty}(\exp (i u x)-1-i u x) \nu(d x)\right)\right\} .
$$

É interessante a utilização desta representação pois a taxa de decaimento exponencial das caudas da medida de Lévy pode ser muito pequena (decaimento devagar), sendo que neste caso $\mathbb{E}\left[X_{t}\right]=\gamma_{c} t$. Para calcular a função característica, considere a parte positiva do domínio da medida de Lévy e suponha que $\alpha \neq 1$ e $\alpha \neq 0$. Assim,

$$
\begin{aligned}
\Psi(u)= & \int_{0}^{\infty}(\exp \{i u x\}-1-i u x) \frac{\exp \{-\lambda x\}}{x^{1+\alpha}} d x \\
= & \int_{0}^{\infty} \frac{\exp \{(i u-\lambda) x\}}{x^{1+\alpha}} d x-\int_{0}^{\infty} \frac{\exp \{-\lambda x\}}{x^{1+\alpha}} d x-i u \int_{0}^{\infty} \frac{\exp \{-\lambda x\}}{x^{\alpha}} d x \\
= & (\lambda-i u)^{\alpha} \int_{0}^{\infty} \exp \{-z\} z^{(-1-\alpha)} d z \\
& -\lambda^{\alpha} \int_{0}^{\infty} \exp \{-z\} z^{(-1-\alpha)} d z-i u \lambda^{\alpha-1} \int_{0}^{\infty} \exp \{-z\} z^{-\alpha} d z \\
= & (\lambda-i u)^{\alpha} \Gamma[-\alpha]-\lambda^{\alpha} \Gamma[-\alpha]-i u \lambda^{\alpha-1} \Gamma[1-\alpha] \\
= & \lambda^{\alpha} \Gamma[-\alpha]\left[\left(1-\frac{i u}{\lambda}\right)^{\alpha}-1+\frac{i u \alpha}{\lambda}\right]
\end{aligned}
$$


No caso de $\alpha=1$, tem-se que

$$
\begin{aligned}
\Psi(u) & =\lim _{\alpha \rightarrow 1} \lambda^{\alpha} \Gamma[-\alpha]\left[\left(1-\frac{i u}{\lambda}\right)^{\alpha}-1+\frac{i u \alpha}{\lambda}\right] \\
& =\lim _{\alpha \rightarrow 0} \lambda^{\alpha+1} \alpha^{-1}\left[\left(1-\frac{i u}{\lambda}\right)^{\alpha+1}-1+\frac{i u(\alpha+1)}{\lambda}\right] \\
& =(\lambda-i u) \ln \left(1-\frac{i u}{\lambda}\right)+i u,
\end{aligned}
$$

e no caso de $\alpha=0$,

$$
\begin{aligned}
\Psi(u) & =\lim _{\alpha \rightarrow 0} \lambda^{\alpha} \Gamma[-\alpha]\left[\left(1-\frac{i u}{\lambda}\right)^{\alpha}-1+\frac{i u \alpha}{\lambda}\right] \\
& =\frac{u}{i \lambda}+\ln \left(\frac{i \lambda}{u+i \lambda}\right) .
\end{aligned}
$$

Por meio destes resultados é possível somar as partes do eixo positivo e negativo da medida de Lévy de um processo estável temperado generalizado.

Proposição 3.5 Seja $\left\{X_{t}\right\}_{t>0}$ um processo estável temperado generalizado. No caso de geral $\left(\alpha_{ \pm} \neq 1\right.$ e $\alpha_{ \pm} \neq 0$ ) o expoente característico $\Psi(u)=t^{-1} \log \mathbb{E}\left[\exp \left\{\right.\right.$ iu $\left.\left.X_{t}\right\}\right]$ é igual a

$$
\begin{aligned}
\Psi(u)=i u \gamma_{c} & +\Gamma\left[-\alpha_{+}\right] \lambda_{+}^{\alpha_{+}} c_{+}\left\{\left(1-\frac{i u}{\lambda_{+}}\right)^{\alpha_{+}}-1+\frac{i u \alpha_{+}}{\lambda_{+}}\right\} \\
& +\Gamma\left[-\alpha_{-}\right] \lambda_{-}^{\alpha_{-}} c_{-}\left\{\left(1-\frac{i u}{\lambda_{-}}\right)^{\alpha_{-}}-1+\frac{i u \alpha_{-}}{\lambda_{-}}\right\} .
\end{aligned}
$$

Se $\alpha_{ \pm}=1$

$$
\begin{gathered}
\Psi(u)=i u\left(\gamma_{c}+c_{+}-c_{-}\right)+c_{+}\left(\lambda_{+}-i u\right) \ln \left(1-\frac{i u}{\lambda_{+}}\right) \\
+c_{-}\left(\lambda_{-}-i u\right) \ln \left(1-\frac{i u}{\lambda_{-}}\right)
\end{gathered}
$$

e se $\alpha_{ \pm}=0$

$$
\begin{aligned}
\Psi(u)=i u \gamma_{c} & -c_{+}\left\{-\frac{i u}{\lambda_{+}}+\ln \left(1-\frac{i u}{\lambda_{+}}\right)\right\} \\
-c_{-} & \left\{-\frac{i u}{\lambda_{-}}+\ln \left(1-\frac{i u}{\lambda_{-}}\right)\right\} .
\end{aligned}
$$

Os outros casos, i.e., quando os $\alpha$-s são iguais a 1 ou 0 , podem ser obtidos de maneira similar. 
Conforme Proposição 2.13, tem-se que:

$$
\begin{aligned}
& \mathbb{E}\left[X_{t}\right]=\kappa_{0}= t \gamma_{c}, \\
& \mathbb{V}\left[X_{t}\right]=\kappa_{2}= t \Gamma\left[2-\alpha_{+}\right] c_{+} \lambda_{+}^{\alpha_{+}-2}+t \Gamma\left[2-\alpha_{-}\right] c_{-} \lambda_{-}^{\alpha_{-}-2}, \\
& \kappa_{3}= t \Gamma\left[3-\alpha_{+}\right] c_{+} \lambda_{+}^{\alpha_{+}-3}+t \Gamma\left[3-\alpha_{-}\right] c_{-} \lambda_{-}^{\alpha_{-}-3} \\
& \kappa_{4}=t \Gamma\left[4-\alpha_{+}\right] c_{+} \lambda_{+}^{\alpha_{+}-4}+t \Gamma\left[4-\alpha_{-}\right] c_{-} \lambda_{-}^{\alpha_{-}-4} .
\end{aligned}
$$

De uma maneira geral, $\lambda_{-}$e $\lambda_{+}$determinam o comportamento das caudas referente a parte negativa e positiva do eixo dos $\mathbb{R}$, respectivamente, da medida de Lévy. Eles indicam o quão freqüente ocorrem saltos grandes e, sob o ponto de vista de análise de risco, isto representa o quanto de dinheiro é possível perder (ou ganhar) em um intervalo de tempo pequeno. Os parâmetros $c_{-}$e $c_{+}$determinam a freqüência de ocorrência de saltos negativos e positivos, respectivamente, sejam estes grandes ou pequenos. Por último, $\alpha_{-}$e $\alpha_{+}$representam o comportamento local do processo, i.e., como os preços se comportam entre a ocorrência dos saltos grandes. Quando $\alpha_{-}$e $\alpha_{+}$são próximo de 2, o comportamento do processo tende a parecer com um movimento Browniano, com muitas oscilações pequenas entre os grandes saltos. Por outro lado, quando $\alpha_{-}$ e $\alpha_{+}$são próximos de zero, as mudanças nos preços são decorrentes essencialmente pela ocorrência dos grandes saltos com períodos de tranquilidade entre estes. Quando $\alpha_{-}, \alpha_{+}<1$ as trajetórias são de variação finita e quando $\alpha_{-}, \alpha_{+}<0$ a medida de Lévy é de atividade finita.

O excesso de curtose para distribuições estáveis simétricas [Mardia et al. (1980), p.49], i.e., $\alpha_{+}=\alpha_{-}, \lambda_{+}=\lambda_{-}$e $c_{+}=c_{-}$, que é igual a:

$$
\alpha_{4}\left[X_{t}\right]=\frac{(3-\alpha)(2-\alpha)}{2 \Gamma[2-\alpha] t c \lambda^{\alpha}},
$$

permite observar que o excesso de curtose pode ser reduzido ou à medida que aumentase a taxa de decaimento das caudas $\lambda$ (reduz a ocorrência de grandes saltos), ou quando o processo é contínuo $\alpha=2$, ou quando aumenta a freqüência dos saltos (grandes e pequenos) $c$. 


\subsection{Modelo Hiperbólico Generalizado}

Outra abordagem para construção de processos de Lévy é pela determinação direta da densidade de probabilidade. Considere o subordinador gaussiano invertido apresentado no Quadro 3.3 com a seguinte parametrização:

$$
p(x)=c(\chi, \zeta) x^{-3 / 2} \exp \left\{-\frac{1}{2}\left(\chi x-\frac{\zeta}{x}\right)\right\} 1_{\{x>0\}} .
$$

Ao introduzir um parâmetro adicional nesta distribuição, obtém-se a distribuição denominada gaussiana invertida generalizada (GIG):

$$
p(x)=c(\lambda, \chi, \zeta) x^{\lambda-1} \exp \left\{-\frac{1}{2}\left(\chi x-\frac{\zeta}{x}\right)\right\} 1_{\{x>0\}} .
$$

Conforme foi provado por Halgreen (1979), a distribuição GIG é infinitamente divisível e pode gerar um processo de Lévy (subordinador). Entretanto, a convolução de distribuições GIG não está fechada em uma distribuição GIG. Seja $S$ uma v.e. GIG e $W$ uma v.e gaussiana padronizada independente. Assim, a distribuição $\sqrt{S} W+\mu S$, sendo que $\mu$ é uma constante, é uma mistura da média-variância da distribuição gaussiana com GIG. Isto pode ser visto como uma subordinação estática do MB, i.e., a distribuição do processo subordinado em um instante de tempo $t$ é uma mistura de média-variância, sendo que a distribuição de mistura é a distribuição do subordinador no instante $t$. A classe de distribuições resultantes destas misturas é denominada hiperbólica generalizada $(\mathrm{GH})$ e também são distribuições infinitamente divisíveis [Halgreen (1979)].

Aplicações em finanças foram realizadas por: Eberlein et al. (1998) que discute as hipóteses do modelo BSM e aplicações do modelo GH na gestão de risco; Prause (1999), que aplicou o modelo GH no apreçamento de opções; Eberlein \& Prause (2000) e Eberlein (2001) discutiram as vantagens do modelo GH na modelagem de séries temporais de preços de ativos financeiros em relação ao MB. No Brasil, Fajardo et al. (2001) e Fajardo \& Farias (2004) buscaram estimar os parâmetros da distribuição GH univariada com dados brasileiros para o período de jan/1994 a $\operatorname{dez} / 2001$. 
A distribuição GH univariada possui cinco parâmetros e é geralmente definida via densidade de Lebesgue:

$$
\begin{aligned}
p(x ; \lambda, \alpha, \beta, \delta, \mu)= & C\left(\delta^{2}+(x-\mu)^{2}\right)^{\lambda / 2-1 / 4} \\
& \times K_{\lambda-1 / 2}\left(\alpha \sqrt{\delta^{2}+(x-\mu)^{2}}\right) \exp \{\beta(x-\mu)\} \\
C= & \left(\frac{\alpha^{2}-\beta^{2}}{\alpha^{2}-(\beta+i u)^{2}}\right)^{\lambda / 2} \frac{K_{\lambda}\left(\delta \sqrt{\lambda^{2}-(\beta+i u)^{2}}\right)}{K_{\lambda}\left(\alpha^{2}-\beta^{2}\right)}
\end{aligned}
$$

sendo que, $K$ é uma função Bessel modificada do segundo tipo. Segundo Cont \& Tankov (2004b) [cap.4, p.125], a função característica desta distribuição é:

$$
\Phi(u)=\exp \{i \mu u\}\left(\frac{\alpha^{2}-\beta^{2}}{\alpha^{2}-(\beta+i u)^{2}}\right)^{\lambda / 2} \frac{K_{\lambda}\left(\delta \sqrt{\lambda^{2}-(\beta+i u)^{2}}\right)}{K_{\lambda}\left(\delta \sqrt{\alpha^{2}-\beta^{2}}\right)} .
$$

A principal desvantagem das distribuições GH é que elas não são fechadas sob convoluções, i.e., a soma de duas variáveis estocásticas independentes GH não é uma v.e. GH. Assim, a utilização de distribuições GH é incoveniente para modelar dados com escalas temporais diferentes. Por outro lado, é relativamente fácil simular uma amostra GH e estimar os parâmetros quando os dados encontram-se na mesma escala temporal. Como a distribuição GH é infinitamente divisível, é possível construir um processo de Lévy hiperbólico generalizado com distribuições em instantes de tempo específicos igual a $\Phi(u)^{t}$. Segundo Prause (1999) [Cap.1, p.22] e Raible (2000) [Cap.2, p.30] a representação de Lévy-Khinchin de um processo de Lévy hiperbólico generalizado para $\lambda \geq 0$ é

$$
\begin{aligned}
\Psi(u)= & i u \mu+\int(\exp \{i u x\}-1-i u x) \nu(x) \\
\nu(x)= & \frac{\exp \{\beta x\}}{|x|}\left(\int_{0}^{\infty} \frac{\exp \left\{-\sqrt{2 y+\alpha^{2}}|x|\right\}}{\pi^{2}\left[y J_{\lambda}^{2}(\delta \sqrt{2 y})+Y_{\lambda}^{2}(\delta \sqrt{2 y})\right]} d y\right. \\
& +\lambda \exp \{-\alpha|x|\}) .
\end{aligned}
$$

Lema 3.1 Seja $X=\left\{X_{t}\right\}_{t \geq 0}$ um processo de Lévy com expoente característico $\Psi(u)=\frac{1}{t} \ln \left(\mathbb{E}\left[\exp \left\{i u X_{t}\right\}\right]\right)$ e seja $\tilde{\Psi}(u)=\frac{\Psi(u)+\Psi(-u)}{2}$ o expoente característico simetrizado. 
1. Se X é um processo composto de Poisson, então, $\tilde{\Psi}(u)$ é uma função com limitada.

2. Se X é um processo de variação finita, então, $\frac{\tilde{\Psi}(u)}{u} \longrightarrow 0$ a medida que $u \longrightarrow \infty$.

3. Se $X$ não possuir o componente $M B$, então, $\frac{\tilde{\Psi}(u)}{u^{2}} \longrightarrow 0$ a medida que $u \longrightarrow \infty$.

Prova A partir da representação de Lévy-Khinchin tem-se que:

$$
\tilde{\Psi}(u)=-\frac{1}{2} \sigma^{2} u^{2}-\int_{-\infty}^{\infty}(1-\cos (u x)) \nu(d x) .
$$

1. Se $X$ é um processo composto de Poisson, então, pela Proposição $2.8, \sigma^{2}=0$ e $\int_{-\infty}^{\infty} \nu(d x)<\infty$. Como

$$
\begin{aligned}
|\tilde{\Psi}(u)| & =\left|-\frac{1}{2} \sigma^{2} u^{2}-\int_{-\infty}^{\infty}(1-\cos (u x)) \nu(d x)\right| \\
& =\frac{1}{2} \sigma^{2} u^{2}+\int_{-\infty}^{\infty}(1-\cos (u x)) \nu(d x) \\
& =\int_{-\infty}^{\infty}(1-\cos (u x)) \nu(d x) \leq 2 \int_{-\infty}^{\infty} \nu(d x)<\infty,
\end{aligned}
$$

então, $\tilde{\Psi}(u)$ é limitado.

2. Se $X$ for um processo de variação finita, então, pela Proposição $2.9, \sigma^{2}=0$ e $\int_{-1}^{1}|x| \nu(d x)<\infty$. Como

$$
\begin{aligned}
|\tilde{\Psi}(u)| & =\left|\int_{-\infty}^{\infty}(1-\cos (u x)) \nu(d x)\right| \\
& \leq \int_{-\infty}^{\infty}|(1-\cos (u x))| \nu(d x) \leq \int_{-\infty}^{\infty}|z| \nu(d x),
\end{aligned}
$$

então,

$$
|(1-\cos (u x))| \leq|z|, z \in \mathbb{R}
$$

Assim, tem-se que

$$
\lim _{u \uparrow \infty} \int_{-\infty}^{\infty} \frac{1-\cos (u x)}{u} \nu(d x)=\int_{-\infty}^{\infty} \lim _{u \uparrow \infty} \frac{1-\cos (u x)}{u} \nu(d x)=0 .
$$

3. Se $X$ não possuir o componente do $\mathrm{MB}$, i.e., $\Sigma=0$, tem-se que $|(1-\cos (u x))| \leq$ $z^{2}, z \in \mathbb{R}$, o que implica em

$$
\int_{-\infty}^{\infty} \lim _{u \uparrow \infty} \frac{1-\cos (u x)}{u^{2}} \nu(d x)=0 .
$$


Quadro 3.5. Propriedades do modelo hiperbólico generalizado.

\begin{tabular}{|l|l|}
\hline Tipo de Modelo & Processo de variação infinita sem o componente MB (caso geral). \\
\hline Parâmetros & $\begin{array}{l}5: \delta \text { é o parâmetro de escala, } \mu \text { é o parametro de deslocamento, } \\
\lambda, \alpha \text { e } \beta \text { determinam a forma da distribuição. }\end{array}$ \\
\hline $\begin{array}{l}\text { Médida de } \\
\text { Lévy } \nu(x)\end{array}$ & Equação 3.44. \\
\hline $\begin{array}{l}\text { Função Carac- } \\
\text { terística } \Phi(u)\end{array}$ & Equação 3.43. \\
\hline $\begin{array}{l}\text { Densidade de Pro- } \\
\text { babilidade } p(x)\end{array}$ & $\begin{array}{l}\text { Equação } 3.42 . \\
\begin{array}{l}\text { Cumulantes: } \\
E\left[X_{t}\right]\end{array}\end{array}$ \\
$V\left[X_{t}\right]$ & $\left.\begin{array}{l}\mu+\frac{\mu \delta}{\sqrt{\alpha^{2}-\beta^{2}}} \frac{K_{\lambda+1}(\zeta)}{K_{\lambda}(\zeta)}, \text { sendo que } \zeta=\delta \sqrt{\alpha^{2}-\beta^{2}} \\
\delta^{2}\left(\frac{K_{\lambda+1}(\zeta)}{\zeta K_{\lambda}(\zeta)}+\frac{\beta^{2}}{\alpha^{2}-\beta^{2}}\left\{\frac{K_{\lambda+2}(\zeta)}{K_{\lambda}(\zeta)}-\left(\frac{K_{\lambda+1}(\zeta)}{K_{\lambda}(\zeta)}\right)^{2}\right\}\right)\end{array}\right)$ \\
\hline $\begin{array}{l}\text { Comportamento } \\
\text { das caudas }\end{array}$ & $\begin{array}{l}\text { No caso geral, tanto a medida de Lévy como a densidade de } \\
\text { probabilidade possuem decaimento exponencial com taxa de } \\
\text { decaimento } \lambda_{+}=\alpha-\beta \text { e } \lambda_{-}=\alpha+\beta .\end{array}$ \\
\hline
\end{tabular}

Para a distribuição GH pode-se utilizar as propriedades assintóticas da função Bessel [Apêndice E] e verificar que $\tilde{\Psi}(u) \sim \delta|u|$, para $u \uparrow \infty$. Esta relação é válida de uma maneira geral, entretanto, casos particulares $\operatorname{como} \delta=0$ a identidade não vale. Isto significa que, exceto em casos particulares, a medida de Lévy de um processo de Lévy hiperbólico generalizado sem o componente MB possui variação infinita. A principal vantagem da distribuição GH é a possibilidade de assumir diversas formas. O Quadro 3.6 [Prause (1999), Cap. 1, p.4] sumariza algumas formas particulares da distribuição GH e o Quadro 3.5 sumariza as principais propriedades desta distribuição.

Exemplo 3.3 O Quadro 3.7 sumariza as principais características de 11 séries temporais brasileiras de ativos financeiros. Calcule o retorno em um instante de tempo pelo logarítimo da razão dos preços, i.e., $\ln \left(p_{t} / p_{t-1}\right)$. Considere o método de estimação de máxima verossimilhança

$$
\mathcal{L}=\sum_{i=1}^{n} \ln (p(x ; \lambda, \alpha, \beta, \delta, \mu)),
$$


Quadro 3.6. Casos particulares da distribuição hiperbólica generalizada.

\begin{tabular}{|l|l|}
\hline Distribuição & Parâmetros do modelo GH \\
\hline Normal & $\alpha, \delta \rightarrow \infty, \delta / \alpha \rightarrow \sigma^{2}$ \\
\hline NIG & $\lambda=-1 / 2$ \\
\hline VG & $\delta=0$ e $\mu=0$. \\
\hline t de Student & $\lambda<0, \alpha=\beta=\mu=0$ \\
\hline Cauchy & $\lambda=-1 / 2, \alpha=\beta=\mu=0$ \\
\hline
\end{tabular}

sendo que $p(x ; \lambda, \alpha, \beta, \delta, \mu)$ é a p.d.f. apresentada na equação (3.42) e $n$ é o tamanho da série temporal. Quando $\lambda=1$ obtém a classe de distribuições hiperbólicas, sendo definida como:

$$
\operatorname{hyp}(x ; \alpha, \beta, \delta, \mu)=\frac{\sqrt{\alpha^{2}-\beta^{2}}}{2 \delta \alpha K_{1}\left(\delta \sqrt{\alpha^{2}-\beta^{2}}\right)} \exp \left\{-\alpha \sqrt{\delta^{2}+(x-\mu)^{2}}+\beta(x-\mu)\right\},
$$

sendo que $x, \mu \in \mathbb{R}, 0 \leq \delta$ e $|\beta|<\alpha$. Assim, pelo método de integração numérica Gauss-Kronrod Quadrature [Ueberhuber (1997), p.105] é possível obter as estimativas apresentadas no Quadro 3.8. Fajardo \& Farias (2004) realizaram os testes econométricos, tais como, Qui-quadrado, Distâncias de Kolmogorov, Kupiec e Anderseon \& Darling e verificaram maior aderência aos dados empíricos da distribuição hiperbólica quando comparada com a distribuição gaussiana, para séries temporais de ações brasileiras. A Figura 3.1 permite visualizar a diferença entre a distribuição gaussiana e hiperbólica para os parâmetros estimados pelo método de máxima verossimilhança. Cabe observar que as estimativas foram efetuadas com dados diários e a taxa de juros Selic apresenta flutuações nos preços em bases mensais, o que prejudicou bastante as estimativas dos parâmetros (tanto da distribuição gaussiana como hiperbólica). 
Figura 3.1. Histograma e p.d.f. da distribuição gaussiana e hiperbólica com parâmetros obtidos pelo método de máxima verossimilhança de 11 ativos financeiros brasileiros.
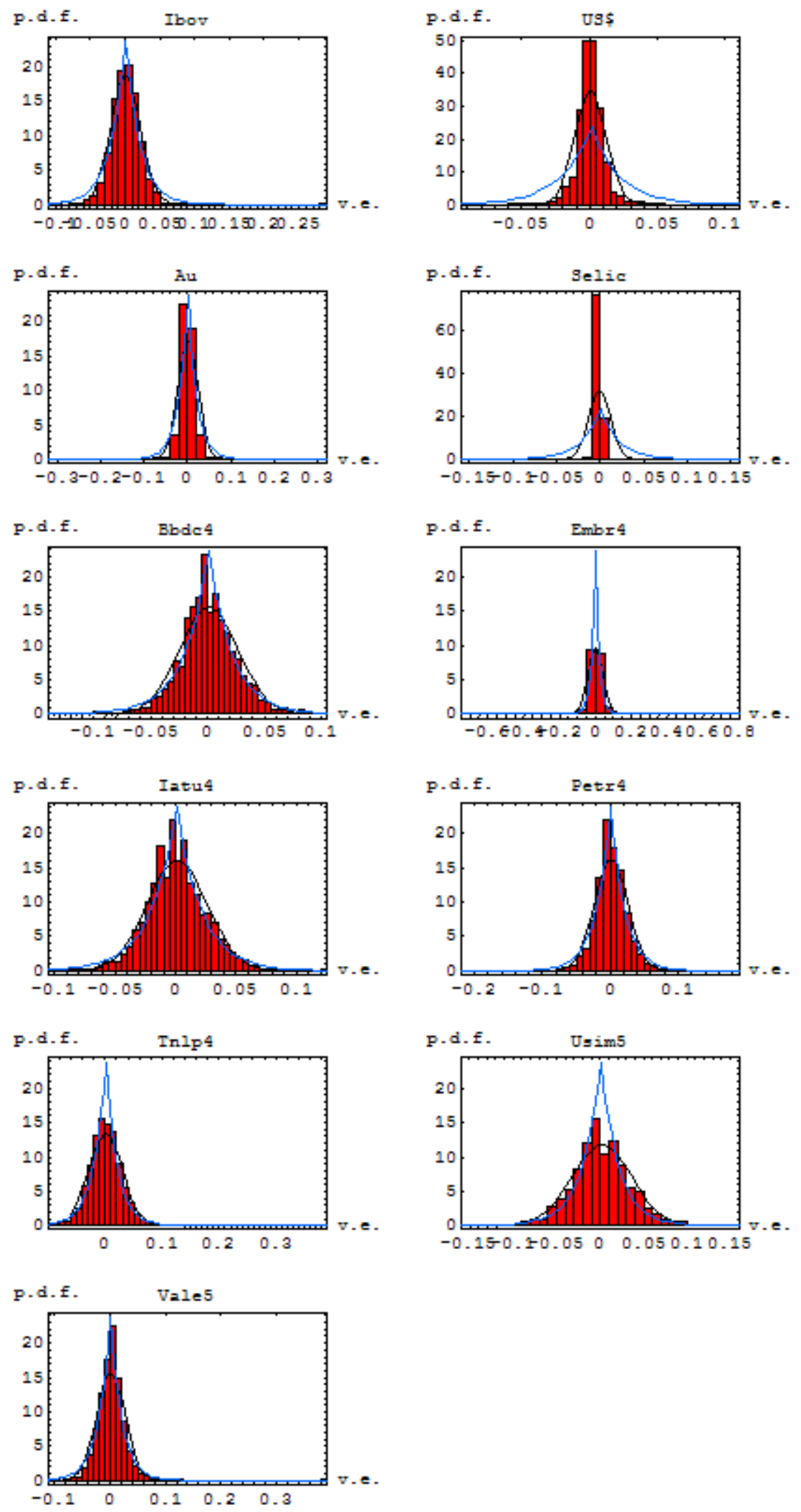
Quadro 3.7. Características das séries temporais de 11 ativos financeiros brasileiros.

\begin{tabular}{|c|c|c|c|c|}
\hline Ticker & Descrição & Fonte & Início & Fim \\
\hline Ibov & Índice Ibovespa & Reuters & 31.12 .1998 & 31.12 .2004 \\
US\$ & Dólar comercial - venda & Sisbacen PTAX800 & 31.12 .1998 & 31.12 .2004 \\
Au & Ouro - 1 grama & BM\&F & 31.12 .1998 & 31.12 .2004 \\
Selic & Taxa de Juros Selic & Sisbacen PTAX860 & 31.12 .1998 & 31.12 .2004 \\
Bbdc4 & Bradesco - PN & Reuters & 31.12 .1998 & 31.12 .2004 \\
Embr4 & Embraer & Reuters & 31.12 .1998 & 31.12 .2004 \\
Itau4 & Itaú - PN & Reuters & 31.12 .1998 & 31.12 .2004 \\
Petr4 & Petrobrás - PN & Reuters & 31.12 .1998 & 31.12 .2004 \\
Tnlp4 & Telemar - PN & Reuters & 31.12 .1998 & 31.12 .2004 \\
Usim5 & Usiminas - PN & Reuters & 31.12 .1998 & 31.12 .2004 \\
Vale5 & Vale do Rio Doce - PNA & Reuters & 31.12 .1998 & 31.12 .2004 \\
\hline
\end{tabular}

Quadro 3.8. Estimação dos parâmetros de 11 ativos financeiros brasileiros pelo método de máxima verossimilhança para a classe de distribuições hiperbólicas $(\lambda=1)$. A esquerda o resultado ótimo da função de verssimilhança $\mathcal{L}$ dado. E as colunas a direita os parâmetros estimados.

\begin{tabular}{|c|c|r|r|r|}
\hline Ticker & $\alpha$ & \multicolumn{1}{|c|}{$\beta$} & \multicolumn{1}{|c|}{$\delta$} & \multicolumn{1}{|c|}{$\mu$} \\
\hline Ibov & 48,2594 & $-1,24193$ & $2.86502660 \times 10^{-8}$ & 0.00132982 \\
US\$ & 33,5537 & 3,23464 & $6,01173561 \times 10^{-5}$ & -0.00215234 \\
Au & 35,6637 & 0.18452 & $5.03060515 \times 10^{-8}$ & 0.00284642 \\
Selic & 94,6533 & $-1,03900$ & $8.65929464 \times 10^{-8}$ & 0.00014194 \\
Bbdc4 & 46,3819 & 1,44441 & $6,47650151 \times 10^{-5}$ & 0.00198179 \\
Embr4 & 33,1307 & $-1,47545$ & $5,79409129 \times 10^{-4}$ & 0.00239407 \\
Itau4 & 51,7688 & 2,68383 & $2,21745083 \times 10^{-3}$ & 0.00110329 \\
Petr4 & 35,1662 & $-1,62952$ & $9,43258199 \times 10^{-5}$ & 0.00215916 \\
Tnlp4 & 32,9636 & $-1,28806$ & $9.04613013 \times 10^{-6}$ & 0.00335502 \\
Usim5 & 39,2418 & 1,91398 & $3.82613292 \times 10^{-8}$ & 0.00012969 \\
Vale5 & 59,9020 & 2,01453 & $5,85349682 \times 10^{-3}$ & 0.00096032 \\
\hline
\end{tabular}




\section{Capítulo 4 \\ Modelagem Multidimensional de Processos Estocásticos de Lévy}

Além da precificação e hedge de opções de um único ativo financeiro, existem várias aplicações de modelos multivariados com dependência entre os componentes, e.g., precificação de uma cesta de opções, otimização de carteiras, simulação de cenário de riscos, etc. Adicionalmente, a ocorrência de saltos no processo log-retornos também pode ser levada em consideração. Entretanto, modelos multidimensionais com saltos são mais difíceis de serem construídos que os modelos unidimensionais. Isto gera uma discrepância entre a quantidade de aplicações com modelos unidimensionais e multidimensionais existentes.

Uma das razões para a utilização dos modelos gaussianos, é que a estrutura de dependência destes modelos pode ser parametrizada em termos da matriz de correlação. Neste caso particular, as propriedades marginais, que são dadas pelas volatilidades, podem ser distinguidas das propriedades de dependência, que são dadas pela matriz de correlação. Outra razão para a utilização de modelos gaussianos é a possibilidade de simular séries temporais gaussianas com matrizes de correlação arbitrárias.

Um método para introduzir saltos em um modelo multidimensional é modificar o tempo de MB multidimensional com um subordinador univariado [Eberlein (2001) e Prause (1999)]. Esta abordagem permite construir e simular modelos multidimensionais, tais como os processos variância gama, normal gaussiana invertida e hiperbólico generalizado. Por outro lado, a modelagem da estrutura de dependência se torna um pouco rígida, pois apenas componentes do mesmo tipo podem ser utilizados, i.e., todos os componentes possuem a mesma distribuição marginal.

No caso de modelos de atividade finita, a estrutura de dependência pode ser obtida ao estabelecer dependências entre os saltos individuais produzidos por processos de Poisson unidimensionais, vide Lindskog $\&$ McNeil (2001). Se os saltos forem normais, a dependência pode ser estabelecida pela matriz de correlação. Se os saltos não forem normais, e.g., se assumirem distribuição de Laplace assimétrica, como no modelo de Kou [Kou (2002) e Kou \& Wang (2004)], então, a dependência pode ser modelada por 
cópulas, entretanto, esta abordagem só faz sentido quando a quantidade de fontes de riscos que produzem os saltos é pequena, e.g., quando todos os componentes (processos de preços dos ativos ativos financeiros) do modelo apresentam saltos no mesmo instante de tempo. Quando a quantidade de fontes de risco é grande esta abordagem não é parcimoniosa e levará em consideração uma quantidade muito grande de parâmetros. Outra limitação desta abordagem, é que todos os componentes do modelo devem ser do mesmo tipo, i.e., todos os componentes devem ser processos compostos de Poisson.

Um modelo multidimensional apropriado deve considerar as seguintes propriedades:

1. Especificação de qualquer modelo unidimensional para qualquer componente individual;

2. O leque de possibilidade de estruturas de dependência deve incluir dependência completa e independência, sendo que a transição entre estes dois extremos deve ser harmoniosa;

3. A dependência deve ser parametrizada de forma parcimoniosa, i.e., com poucos parâmetros.

Estas características podem ser obtidas se a dependência for modelada de maneira independente do comportamento marginal dos componentes, i.e., a modelagem da estrutura de dependência de um vetor de variáveis estocásticas pode ser efetuada de maneira segregada das distribuições marginais por meio de cópulas. Apesar deste procedimento ser algo relativamente recente, dois livros que tratam sobre o assunto são: Nelsen (1999), para o caso bivariado e Joe (1997), para o caso multivariado. Estas duas referências consideram variáveis estocásticas em apenas um determinado instante de tempo, sendo que é necessário estender estes conceitos para o ambiente dinâmico dos processos de Lévy, como será abordado adiante neste estudo. A dependência entre os componentes de um processo de Lévy multidimensional pode ser completamente caracterizada por uma cópula de Lévy, i.e., uma função que possui as mesmas propriedade de uma cópula ordinária, entretanto, é definida em um domínio distinto. Este procedimento fornece as ferramentas necessárias para construir processos de Lévy multidimensionais com dependências específicas. Existem várias famílias 
paramétricas de cópulas de Lévy que podem ser utilizadas para construir processos de Lévy de dimensão $n$, ou seja, ao copular $n$ processos de Lévy unidimensionais por meio de uma cópula parametrizada. Apesar dos modelos construídos por meio da abordagem cópula de Lévy descreverem todas as características de dependência multidimensional, eles podem se tornar matematicamente complexos e difíceis de serem computacionalmente implementados.

\subsection{Modelagem via Subordinação Browniana}

Uma possível generalização do modelo BSM é supor que o preço dos ativos financeiros segue um MB multidimensional com escala temporal modificada (estocástica). Em outras palavras, o mercado percebe o tempo de maneira diferente a nossa percepção, algumas vezes ele anda mais rápido que o nosso relógio, outras vezes ele anda mais de devagar. Suponha que a escala temporal do mercado é modelada por um subordinador $Z=\left\{Z_{t}\right\}_{t \geq 0}$ com expoente laplaciano $l(u)$ e que $B=\left\{B_{t}\right\}_{t \geq 0}$ é um MB $d$-dimensional com matriz de covariância $\Sigma$ e $\mu \in \mathbb{R}^{d}$. Ao modificar a escala temporal de um MB com t.d. $\mu$ por meio de $Z$, obtém-se um novo processo de Lévy $d$ dimensional $X_{t}=B\left(Z_{t}\right)+\mu Z_{t}$. Este processo pode ser utilizado para modelar $d$ ativos financeiros dependentes: $S_{t}^{i}=\exp \left\{X_{t}^{i}\right\}$ para $i=1, \ldots, d$. A função característica de $X_{t}$ pode ser calculada por meio do Teorema 3.2, i.e., para cada $u \in \mathbb{R}^{d}$,

$$
\mathbb{E}\left[\exp \left\{i u \cdot X_{t}\right\}\right]=\exp \left\{t l\left(-\frac{1}{2} u \cdot \Sigma u+i \mu . u\right)\right\}
$$

As fórmulas (3.7) e (3.8) permitem calcular o tripé característico de $X=\left\{X_{t}\right\}_{t \geq 0}$. A distribuição gaussiana condicionada do modelo permite a simulação dos incrementos de $X_{t}$. Isto pode ser feito ao simular, incialmente, os incrementos do subordinador $Z$ e, posteriormente, ao simular os incrementos de um MB $d$-dimensional na escala de tempo dado pelos incrementos simulados do subordinador.

Para sentir as propriedades deste modelo, considere um modelo bidimensional. Seja $S^{1}=\left\{S_{t}^{1}\right\}_{t \geq 0}$ e $S^{2}=\left\{S_{t}^{2}\right\}_{t \geq 0}$ dois processos de ativos financeiros, tal que:

$$
\begin{aligned}
& S_{t}^{1}=\exp \left\{X_{t}^{1}\right\}, X_{t}^{1}=B^{1}\left(Z_{t}\right)+\mu_{1} Z_{t}, \\
& S_{t}^{2}=\exp \left\{X_{t}^{2}\right\}, X_{t}^{2}=B^{2}\left(Z_{t}\right)+\mu_{2} Z_{t},
\end{aligned}
$$


sendo que $B^{1}$ e $B^{2}$ são componentes de um MB bidimensional, com variâncias $\sigma_{1}^{2} \mathrm{e}$ $\sigma_{2}^{2}$ e coeficiente de correlação de Pearson $\rho$. Para $i \in\{1,2\}$ tem-se que a variância do retorno $X_{i}$ é dada por:

$$
\begin{aligned}
\mathbb{V}\left[X_{t}^{i}\right] & =\mathbb{V}\left[B_{t}^{i}\left(Z_{t}\right)+\mu_{i} Z_{t}\right] \\
= & \mathbb{V}\left[B_{t}^{i}\left(Z_{t}\right)\right]+\mathbb{V}\left[\mu_{i} Z_{t}\right]+2 \mathbb{C}\left[B_{t}^{i}\left(Z_{t}\right) ; \mu_{i} Z_{t}\right] \\
= & \mathbb{E}\left[\left(B_{t}^{i}\left(Z_{t}\right)-\mathbb{E}\left[B_{t}^{i}\left(Z_{t}\right)\right]\right)^{2}\right]+\mu_{i}^{2} \mathbb{V}\left[Z_{t}\right] \\
& +2 \mu_{i} \mathbb{E}\left[\left(Z_{t}-\mathbb{E}\left[Z_{t}\right]\right)\left(B_{t}^{i}\left(Z_{t}\right)-\mathbb{E}\left[B_{t}^{i}\left(Z_{t}\right)\right]\right)\right] \\
& =\mu_{i}^{2} \mathbb{V}\left[Z_{t}\right]+\mathbb{E}\left[\mathbb{E}\left[\left(B_{t}^{i}\left(Z_{t}\right)-\mathbb{E}\left[B_{t}^{i}\left(Z_{t}\right)\right]\right)^{2} \mid Z_{t}\right]\right] \\
& +2 \mu_{i} \mathbb{E}\left[\mathbb{E}\left[\left(Z_{t}-\mathbb{E}\left[Z_{t}\right]\right)\left(B_{t}^{i}\left(Z_{t}\right)-\mathbb{E}\left[B_{t}^{i}\left(Z_{t}\right)\right]\right) \mid Z_{t}\right]\right] \\
& =\mu_{i}^{2} \mathbb{V}\left[Z_{t}\right]+\sigma_{i}^{2} \mathbb{E}\left[Z_{t}\right] .
\end{aligned}
$$

A covariância entre os log-retornos $X_{1}$ e $X_{2}$ é dada por:

$$
\begin{aligned}
\mathbb{C}\left[X_{t}^{1}, X_{t}^{2}\right]=\mathbb{E}\left[X_{t}^{1} X_{t}^{2}\right]-\mathbb{E}\left[X_{t}^{1}\right] \mathbb{E}\left[X_{t}^{2}\right] \\
\quad=\mathbb{E}\left[B^{1}\left(Z_{t}\right) B^{2}\left(Z_{t}\right)+\mu_{1} B^{2}\left(Z_{t}\right) Z_{t}+B^{1}\left(Z_{t}\right) \mu_{2} Z_{t}\right] \\
\quad+\mu_{1} \mu_{2} \mathbb{E}\left[Z_{t}^{2}\right]-\mathbb{E}\left[B^{1}\left(Z_{t}\right)+\mu_{1} Z_{t}\right] \mathbb{E}\left[B^{2}\left(Z_{t}\right)+\mu_{2} Z_{t}\right] \\
\quad=\mathbb{E}\left[B^{1}\left(Z_{t}\right) B^{2}\left(Z_{t}\right)\right]+\mathbb{E}\left[\mu_{1} B^{2}\left(Z_{t}\right) Z_{t}+B^{1}\left(Z_{t}\right) \mu_{2} Z_{t}\right] \\
\quad+\mu_{1} \mu_{2} \mathbb{E}\left[Z_{t}^{2}\right]-\mathbb{E}\left[B^{1}\left(Z_{t}\right)\right] \mathbb{E}\left[B^{2}\left(Z_{t}\right)\right]-\mathbb{E}\left[B^{1}\left(Z_{t}\right)\right] \mu_{2} \mathbb{E}\left[Z_{t}\right] \\
\quad-\mu_{1} \mathbb{E}\left[Z_{t}\right] \mathbb{E}\left[B^{2}\left(Z_{t}\right)\right]-\mu_{1} \mathbb{E}\left[Z_{t}\right] \mu_{2} \mathbb{E}\left[Z_{t}\right] \\
\quad=\mathbb{E}\left[\mathbb{E}\left[B^{1}\left(Z_{t}\right) B^{2}\left(Z_{t}\right)\right]-\mathbb{E}\left[B^{1}\left(Z_{t}\right)\right] \mathbb{E}\left[B^{2}\left(Z_{t}\right)\right] \mid Z_{t}\right] \\
\quad+\mathbb{E}\left[\mathbb{E}\left[B^{1}\left(Z_{t}\right) \mu_{2}\left(Z_{t}-\mathbb{E}\left[Z_{t}\right]\right)+\mu_{1} B^{2}\left(Z_{t}\right)\left(Z_{t}-\mathbb{E}\left[Z_{t}\right]\right) \mid Z_{t}\right]\right] \\
\quad+\mu_{1} \mu_{2}\left(\mathbb{E}\left[Z_{t}^{2}\right]-\left(\mathbb{E}\left[Z_{t}\right]\right)^{2}\right) \\
\quad=\sigma_{1} \sigma_{2} \rho \mathbb{E}\left[Z_{t}\right]+\mu_{1} \mu_{2} \mathbb{V}\left[Z_{t}\right] .
\end{aligned}
$$

A correlação dos log-retornos $\rho\left(X_{t}^{1}, X_{t}^{2}\right)$ pode ser calculada ao combinar os resultados (4.1) e (4.2), como segue:

$$
\begin{aligned}
\rho\left(X_{1} ; X_{2}\right) & =\frac{\mathbb{C}\left[X_{t}^{1}, X_{t}^{2}\right]}{\left(\mathbb{V}\left[X_{1}\right]\right)^{1 / 2}\left(\mathbb{V}\left[X_{2}\right]\right)^{1 / 2}} \\
& =\frac{\sigma_{1} \sigma_{2} \rho \mathbb{E}\left[Z_{t}\right]+\mu_{1} \mu_{2} \mathbb{V}\left[Z_{t}\right]}{\left(\mu_{1}^{2} \mathbb{V}\left[Z_{t}\right]+\sigma_{1}^{2} \mathbb{E}\left[Z_{t}\right]\right)^{1 / 2}\left(\mu_{2}^{2} \mathbb{V}\left[Z_{t}\right]+\sigma_{2}^{2} \mathbb{E}\left[Z_{t}\right]\right)^{1 / 2}} .
\end{aligned}
$$

Apenas no caso de simetria completa, i.e., $\mu_{1}=\mu_{2}=0$, tem-se que a correlação $\rho\left(X_{t}^{1}, X_{t}^{2}\right)=\rho$, i.e., a correlação dos log-retornos é igual a correlação do MB que 
está sendo subordinado. No caso de assimetria, i.e., $\mu_{1}, \mu_{2} \neq 0$, a correlação dos log-retornos será diferente da correlação do MB que está sendo subordinado. Mesmo quando os $B^{1}$ e $B^{2}$ são independentes, i.e., $\rho=0$, a correlação dos log-retornos é diferente de zero $\rho\left(X_{t}^{1}, X_{t}^{2}\right) \neq 0$, pois $\mathbb{C}\left[X_{t}^{1}, X_{t}^{2}\right]=\mu_{1} \mu_{2} \mathbb{V}\left[Z_{t}\right]$.

Quando houver independência entre $B^{1}$ e $B^{2}$ combinada com simetria $\mu_{1}=\mu_{2}=0$ tem que os log-retornos são independentes apenas linearmente, ou seja, decorrelacionados. Conforme Cont \& Tankov (2004b) [cap.5, p.134], dado que os componentes do MB dependem do mesmo subordinador, os saltos grandes nos preços dos ativos financeiros tendem a ocorrer juntos, o que significa que valores absolutos dos log-retornos serão correlacionados. Assim, quando $\mu_{1}=\mu_{2}=0$ e $\rho=0$, tem-se que

$$
\mathbb{C}\left[\left(X_{t}^{1}\right)^{2} ;\left(X_{t}^{2}\right)^{2}\right]=\sigma_{1} \sigma_{2} \mathbb{V}\left[Z_{t}\right]
$$

o que indica que o quadrado dos log-retornos são correlacionados se o subordinador não for determinístico. Esta característica induz a erros na avaliação de medidas de risco. $\mathrm{O}$ seguinte exemplo ilustra a situação:

Exemplo 4.1 Seja $X=\left\{X_{t}\right\}_{t \geq 0}$ e $Y=\left\{Y_{t}\right\}_{t \geq 0}$ dois processos de Lévy variância gama simétricos e identicamente distribuídos e, suponha que haja interesse na distribuição de $X_{t}+Y_{t}$ para um horizonte de 10 dias úteis, que aproximadamente corresponte a $1 / 25$ avos de um ano ${ }^{12}$. Considere que os parâmetros de $X_{t}$ e $Y_{t}$ são: volatilidade anualizada de $\sigma=0.30 \mathrm{e}$, excesso de curtose igual a $\kappa_{4}\left[X_{t}\right]=3\left(\mathbb{V}\left[X_{t}\right]\right)^{2}$, o que implica que a variância do subordinador é igual a $\kappa=t$. Assim, é possível comparar que $X_{t}$ e $Y_{t}$ quando forem independentes e decorrelacionados. Os processos independentes podem ser obtidos pela subordinação de MB univariados independentes, sendo cada um, subordinado por um subordinador gama independente e, o processos decorrelacionados podem ser obtidos um MB bivariado subordinado por um único subordinador gama. O Quadro 4.1 ilustra as estatísticas obtidas por simulação de Monte Carlo $^{13}$ com $10^{6}$ observações efetuada no Mathematica 5.1, quando os processos $X$ e $Y$ são independentes e decorrelacionados. O que se observa é que no caso dos processos serem decorrelacionados a soma dos processos, $X_{t}+Y_{t}$, é mais leptocúrtica, e

12 O mercado financeiro convenciona que um ano possui 252 dias úteis (d.u.) no ano, ou 21 d.u. no mês.

13 Para simular o subordinador gama foi utilizado o algoritmo 6.8 ("melhor gerador de variáveis gama" [Cont \& Tankov (2004b), cap.6, p.182]). 
Quadro 4.1. Independência versus decorrelação: processos independentes não podem ser modelados por meio de MB multidimensional utilizando um subordinador unidimensional.

\begin{tabular}{|c|c|c|}
\hline $\begin{array}{c}\text { Estatisticas } \\
\text { de } X_{t}+Y_{t}\end{array}$ & $\begin{array}{c}X_{t} \text { e } Y_{t} \\
\text { independentes }\end{array}$ & $\begin{array}{c}X_{t} \text { e } Y_{t} \\
\text { decorrelacionados }\end{array}$ \\
\hline Volatilidade anual & 0,4389 & 0,4389 \\
\hline Excesso de curtose & 1,5716 & 3,1005 \\
\hline Quantil 5\% & $-0,1432$ & $-0,1425$ \\
\hline Quantil 1\% & $-0,2277$ & $-0,2428$ \\
\hline Quantil 0,1\% & $-0,3441$ & $-0,3914$ \\
\hline
\end{tabular}

os quantil que podem significar perdas maiores, quando os processos representarem log-retornos de ativos financeiros. Assim, a gestão de risco de mercado no contexto do Acordo da Basiléia II $^{14}$, um modelo que contempla uma estrutura de dependência inadequada, i.e., considerar apenas a independência linear (decorrelação) e não a independência completa, pode exigir uma maior quantidade de capital e, conseqüentemente um maior custo para a instituição financeira.

Desta maneira, a subordinação de um MB multidimensional permite construir modelos paramétricos baseados em processos de Lévy, entretanto, estes apresentam duas grandes desvantagens. A primeira, é que não é possível estabelece modelos unidimensionais diferentes para os componentes componentes individuais e, a segunda, as possibilidades de estruturas de dependência são limitadas, pois não contemplam independência completa, i.e., os componentes obtidos são sempre parcialmente dependentes.

\subsection{Modelagem via Processos Compostos de Poisson}

Este modelo permite modelar o processo do log-retorno de ativos financeiros $d$ dimensional que contemple crises de mercado. As datas das crises de mercado podem ser modeladas como tempos de chegada de um processo de Poisson $N=\left\{N_{t}\right\}_{t \geq 0}$. As-

14 Disponível em <http://www.bis.org/publ/index.htm $>$. 
sim, é possível estabelecer o seguinte modelo para o log-retorno de $d$ ativos financeiros:

$$
X_{t}^{i}=\mu^{i} t+B_{t}^{i}+\sum_{j=1}^{N_{t}} Y_{j}^{i}, i=1, \ldots, d
$$

sendo que $B=\left\{B_{t}\right\}_{t \geq 0}$ é um MB $d$-dimensional com matriz de covariância $\Sigma$ e $Y=\left\{Y_{j}\right\}_{j \in \mathbb{N}}$ é um vetor estocático $d$-dimensional que determina o tamanho dos saltos dos ativos individuais durante as crises de mercado. Este modelo contém apenas um processo de Poisson $N$ porque está sendo considerado apenas um tipo de risco de ocorrer saltos, que o risco de ocorreu uma crise de mercado global que afeta todos os ativos simultaneamente. Para construir um modelo paramétrico é necessário especificar a distribuição dos saltos dos ativos individualmente durante a ocorrência das crises de mercado, i.e., a distribuição de $Y^{i}$ para todos os $i$ e a estrutura de dependência entre os saltos destes ativos. Apenas para simplificar, considere que a distribuição dos vetores estocásticos $Y_{j}$ é gaussiana, então, é necessário especificar uma matriz de covariância $\Sigma^{\prime}$ e um vetor de esperança $m$. O resultado é uma versão multivariada do modelo de Merton [Merton (1976)]. Se os saltos não forem gaussianamente distribuídos, é necessário estabelecer uma distribuição para cada componente e uma função de cópula que descreva a estrutura de dependência.

É possível estabelecer um modelo que não considera apenas as crises de mercado, mas que leve em consideração as inovações relacionados aos riscos específicos de cada um dos ativos, ou mesmo riscos setoriais, que afetam apenas uma parcela dos ativos. Assim, é necessário incluir diversos processos de Poisson, o que resultará no seguinte modelo:

$$
X_{t}^{i}=\mu^{i} t+B_{t}^{i}+\sum_{k=1}^{M} \sum_{j=1}^{N_{t}^{k}} Y_{j, k}^{i}, i=1, \ldots, d
$$

sendo que $N_{t}^{1}, \ldots, N_{t}^{M}$ são processos de Poisson que conduzem $M$ inovações independentes e, $Y_{j, k}^{i}$ é o tamanho do salto referente ao $i$-ésimo componente após a ocorrência do $j$-ésimo risco do tipo $k$. Os vetores estocásticos $\left\{Y_{j, k}^{i}\right\}_{i=1}^{d}$ são independentes para quaisquer $j$ e $k$. Para definir completamente um modelo paramétrico, é necessário especificar uma distribuição unidimensional para cada componente e tipo de risco, pois tipos diferentes de inovações influenciam o preço de um ativo financeiro de maneira diferente e, uma cópula $d$-dimensional para tipo de inovação. Isto representa uma quantidade $M \times d$ de distribuições unidimensionais e $M$ cópulas $d$-dimensionais a serem deter- 
minadas. Se houver a necessidade de considerar todas as dependências existentes entre as $M$ cópulas, tem-se adicionalmente uma quantidade de $2^{M}-1$ dependências a serem consideradas. Assim, quando a quantidade de inovações referentes a riscos cresce o problema pode se tornar computacionalmente complicado. Desta maneira, é razoável considerar um modelo deste tipo apenas quando a quantidade $M$ de riscos é pequena. Uma discussão mais detalhada sobre este modelo é feita por Lindskog \& McNeil (2001).

\subsection{Cópulas para Variáveis Estocásticas}

A distribuição de um vetor estocástico bidimensional $\{X, Y\}$ é tipicamente descrito por meio de sua função de densidade acumulada (c.d.f.)

$$
F(x, y)=\mathbb{P}[X \leq x, Y \leq y]
$$

A denominação distribuição marginal ou marginal de $\{X, Y\}$ é dada para as distribuições de $X$ e $Y$ separadamente. Estas distribuições marginais podem ser descritas pelas suas funções de distribuições $F_{X}(x)=\mathbb{P}[X \leq x]$ e $F_{Y}(y)=\mathbb{P}[Y \leq y]$ que por sua vez, podem ser obtidas por meio da c.d.f. bidimensional:

$$
F_{X}(x)=F(x, \infty), F_{Y}(y)=F(\infty, y) .
$$

Para conhecer a distribuição de um vetor estocástico bidimensional, não suficiente conhecer as distribuições marginais. Assim, além das distribuições marginais, $F(x, y)$ também contém a informação sobre a estrutura de dependência entre estas distribuições marginais. Dois vetores com as mesmas distribuições marginais, mas com estruturas de dependência diferentes possuem distribuições distintas. Desta maneira, um vetor estocástico com distribuições marginais $F_{X}(x)$ e $F_{Y}(y)$, será igual a $F(x, y)=F_{X}(x) F_{Y}(y)$ se os seus componentes forem independentes e, $F(x, y)=\min \left(F_{X}(x), F_{Y}(y)\right)$ se eles forem completamente dependentes, i.e., um dos componentes é uma função determinística estritamente crescente do outro componente.

A cópula de um vetor estocástico bidimensional é uma função bivariada que caracteriza a estrutura de dependência e não depende das marginais. O par cópula + marginais descreve a distribuição de um vetor estocástico. A c.d.f. pode ser calculada por 
meio da cópula $C(x, y)$ e de suas marginais, i.e., $F(x, y)=C\left(F_{X}(x), F_{Y}(y)\right)$. Se $F_{X}(x)$ e $F_{Y}(y)$ são contínuas, então, a cópula é unica e pode ser calculada por meio da c.d.f. e das marginais: $C(x, y)=F\left(F_{X}^{-1}(x), F_{Y}^{-1}(y)\right)$.

Dizer que a cópula de uma distribuição não depende das marginais significa que ela é invariante a transformações estritamente crescentes dos componentes do vetor estocástico. Assim, para todo par de funções estritamente crescente $f$ e $g$, a cópula de $X$ e $Y$ é a mesma que a cópula de $f(X)$ e $g(Y)$. O termo estrutura de dependência refere-se as características de uma distribuição que não dependem das marginais e, ao combinar esta estrutura às marginais é possível reconstruir inteiramente a distribuição. Uma abordagem entrópica de medida de dependência é sugerida por Joe (1989).

Seja $F(x, y)$ a distribuição de um vetor estocástico. Esta função possui as seguinte propriedades:

1. Para cada retângulo $B=\left[x_{1} ; x_{2}\right] \times\left[y_{1} ; y_{2}\right]$,

$$
F\left(x_{2}, y_{2}\right)-F\left(x_{2}, y_{1}\right)-F\left(x_{1}, y_{2}\right)+F\left(x_{1}, y_{1}\right)=\mathbb{P}[\{X, Y\} \in B] \geq 0 .
$$

2. Para todo $x, F(-\infty, x)=F(x,-\infty)=0$.

A primeira propriedade pode ser entendida como uma generalização da idéia de função crescente para funções de dimensões superiores, o que permite estabelecer as seguintes definições:

Definição 4.1 Seja $S_{1}$ e $S_{2}$ dois intervalos fechados (possivelmente infinitos) de $\overline{\mathbb{R}}=\mathbb{R} \cup\{\infty\} \cup\{-\infty\}$ e, considere a função $F: S_{1} \times S_{2} \longrightarrow \overline{\mathbb{R}}$.

1. F-volume de um retângulo $B=\left[x_{1} ; x_{2}\right] \times\left[y_{1} ; y_{2}\right] \subset S_{1} \times S_{2}$ é definido como

$$
V_{F}(B)=F\left(x_{2}, y_{2}\right)-F\left(x_{2}, y_{1}\right)-F\left(x_{1}, y_{2}\right)+F\left(x_{1}, y_{1}\right) \text {. }
$$

2. $F$ é 2-crescente se para todo retângulo $B$ em seu domínio $\mathcal{D}(F), V_{F}(B) \geq 0$.

3. $F$ é aderente se para todo $x \in S_{1}, F\left(x, \min S_{2}\right)=0$ e para todo $y \in S_{2}$, $F\left(\min S_{1}, y\right)=0$. 
Se uma função bivariada é crescente em cada um de seus argumentos, em geral ela não é 2-crescente, e.g., $F(x, y)=-\frac{1}{x y}$ para qualquer $x, y>0$ é crescente em $x$ e $y$, mas não é 2-crescente. Se uma função for 2-crescente, ela não é necessariamente crescente em cada um dos seus argumentos, e.g., $F(x, y)=x y$ é 2-crescente mas não é crescente nos seus argumentos se $x, y<0$. Apesar disto, se uma função for 2-crescente e aderente, então, ela é crescente nos seus argumentos.

\section{Lema 4.1 Seja F uma função 2-crescente.}

1. Transformações crescentes de variáveis: se as função $f_{1}$ e $f_{2}$ são crescentes, então, $F\left(f_{X}(x), f_{Y}(y)\right)$ é 2-crescente.

2. Transformação por meio de uma função com derivadas positivas: se $F$ é aderente e $f$ é crescente, convexa e satifaz $f(0)=0$, então, a superposição $f \circ F$ é uma função 2-crescente aderente.

Na teoria de cópulas a definição da função de distribuição é:

Definição 4.2 Uma função abstrata é uma função $F: \overline{\mathbb{R}}^{2} \longrightarrow[0 ; 1]$ aderente, 2 -crescente e satisfaz a condição $F(\infty, \infty)=1$.

Adicionalmente à estas propriedades, a função de distribuição apresentada em (4.3) deve apresentar a seguinte propriedade de continuidade: para todo $x_{0}, y_{0} \in \mathbb{R}$,

$$
\lim _{x \downarrow x_{0}, y \downarrow y_{0}} F(x, y)=F\left(x_{0}, y_{0}\right) .
$$

Segundo Cont \& Tankov (2004b) [cap.5, p.138], é possível mostrar que para cada função abstrata $F$ existe uma única função de distribuição $\tilde{F}$, tal que $V_{F}(B)=V_{\tilde{F}}(B)$ para todo retângulo contínuo $B$ de $F$; sendo que a continuidade do retângulo é definida como um retângulo $B$ para o qual toda seqüência de retângulos convergem para os vértices de $B$; a seqüência de $F$-volumes destes retângulos converge para $V_{F}(B)$. Assim, o termo função asbtrata será substituído por função de distribuição. Ao generalizar a idéia de marginais de uma função de distribuição para qualquer $F: S_{1} \times S_{2} \longrightarrow \overline{\mathbb{R}}$, as funções $F_{X}(x)=F\left(x, \max S_{2}\right)$ e $F_{Y}(x)=F\left(\max S_{1}, y\right)$ são denominadas marginais de $F$. 
Definição 4.3 Cópula Uma cópula bidimensional é uma função $C$ com domínio $\mathcal{D}(C)=[0 ; 1]^{2}$, tal que:

1. C é aderente e 2-crescente.

2. C possui marginais $C_{k}, k=\{1,2\}$, e satisfaz $C_{k}(u)=u$ para todo $u \in[0 ; 1]$.

Sob uma perspectiva probabilística, esta definição significa que uma cópula bidimensional é uma função de distribuição em $[0 ; 1]^{2}$ com marginais uniformes. Assim segue o seguinte teorema:

Teorema 4.1 (Sklar) Seja F uma função de distribuição bidimensional com marginais $F_{1}$ e $F_{2}$. Assim, existe uma cópula bidimensional $C$, tal que para todo $x \in \overline{\mathbb{R}}^{2}$,

$$
F\left(x_{1}, x_{2}\right)=C\left(F_{1}\left(x_{1}\right), F_{2}\left(x_{2}\right)\right) .
$$

Se $F_{1}$ e $F_{2}$ são contínuas $C$ é única, caso contrário, $C$ apresentará domínio $\mathcal{D}(C)$ determinado pelo produto cartesiano das imagens $\mathcal{I}\left(F_{1}\right) \times \mathcal{I}\left(F_{2}\right)$. De maneira contrária, se $C$ é uma cópula e $F_{1}$ e $F_{2}$ são funções de distribuições, então, a função $F$ definida pela equação (4.4) é uma função de distribuição bidimensional com marginais $F_{1}$ e $F_{2}$.

Prova Suponha que $F_{X}$ e $F_{Y}$ sejam contínuas (uma prova para o caso geral pode ser obtida em Kallsen \& Tankov (2005)). Como $F_{X}$ é contínua, é possível achar uma função $F_{X}^{-1}(u)$ que satisfaça $F_{X}^{-1}(0)=-\infty$ e $F_{X}^{-1}(1)=+\infty$, sendo que $F_{X}\left(F_{X}^{-1}(u)\right)=u$ para todo $u \in[0 ; 1]$. A função inversa de $F_{Y}$ pode ser escolhida da mesma maneira. Seja $C(u, v)=F\left(F_{X}^{-1}(u), F_{Y}^{-1}(v)\right)$. Assim, a Propriedade 1 do Lema 4.1 $C$ é 2-crescente. Adicionalmente, $C(0, v)=F\left(F_{X}^{-1}(0), F_{Y}^{-1}(v)\right)=F\left(-\infty, F_{Y}^{-1}(v)\right)=0$ e, $C(1, v)=F\left(F_{X}^{-1}(1), F_{Y}^{-1}(v)\right)=F\left(\infty, F_{Y}^{-1}(v)\right)=F_{Y}\left(F_{Y}^{-1}(v)\right)=v$. Assim, $C$ é uma cópula. Para mostrar a unicidade, suponha a existência de outra cópula $\tilde{C}$ que corresponde a mesma distribuição. Assim, para todo $x, y \in \overline{\mathbb{R}}$, $C\left(F_{X}(x), F_{Y}(y)\right)=\tilde{C}\left(F_{X}(x), F_{Y}(y)\right)$. Desta maneira, pela continuidade de $F_{X}$ e $F_{Y}$, é possível concluir que para todo $u \in[0 ; 1]$ e $v \in[0,1], C(u, v)=\tilde{C}(u, v)$. Em 
relação à observação contrária, pela Propriedade 1 do Lema 4.1, a função definida em (4.4) é 2-crescente. É possível por substituição que $F(x, y)$ é uma função aderente e possui marginais $F_{X}$ e $F_{Y}$ e, que $F(1,1)=1$, o implica que $F(x, y)$ é uma função de distribuição.

\subsubsection{Exemplos de Cópulas}

1. Se $X$ e $Y$ são independentes, a cópula de $\{X, Y\}$ é $C_{\perp}(u, v)=u v$. Se as distribuições de $X$ e/ou $Y$ não forem contínuas, a cópula não é única, logo, $C_{\perp}$ não é a única possibilidade de cópula independente ${ }^{15}$.

2. Se $X=f(Y)$ para certa função determinística estritamente crescente $f$, então, $C_{\|}(u, v)=\min (u, v)$. Esta cópula é denominada cópula de completa dependência ou limite superior de Fréchet, pois para toda $C$ tem-se que $C(u, v) \leq C_{\|}(u, v)$, $\forall u, v$.

3. Se $X=f(Y)$ para certa função determinística estritamente decrescente $f$, então, $C(u, v)=\max (x+y-1,0)$. Este é o caso da cópula completamente negativamente dependente ou limite inferior de Fréchet.

4. A família Clayton de cópulas é uma família determinada por apenas um parâmetro:

$$
C_{\theta}(x, y)=\left(x^{-\theta}+y^{-\theta}-1\right)^{-1 / \theta}, \theta>0 .
$$

A família de Clayton inclui cópulas, tais como de dependência completa ou independência completa, em casos limites:

$$
\begin{aligned}
& C_{\theta} \longrightarrow C_{\|}, \text {quando } \theta \longrightarrow \infty \\
& C_{\theta} \longrightarrow C_{\perp}, \text { quando } \theta \longrightarrow 0
\end{aligned}
$$

5. A família gaussiana de cópulas corresponde à estrutura de dependência de um vetor estocástico gaussiano:

$$
C_{\rho}(x, y)=\mathcal{N}_{2, \rho}\left(\mathcal{N}^{-1}(x), \mathcal{N}^{-1}(y)\right)
$$

15 Esta observação é válida para todos os exemplos a seguir. 
sendo que $\mathcal{N}_{2, \rho}$ é a função de distribuição de uma gaussiana padronizada bivariada com correlação $\rho$ e $\mathcal{N}$ é uma função de distribuição de uma gaussiana padronizada univarida. O caso de $\rho=1$ corresponde a dependência completa e $\rho=0$ corresponde a independência completa.

A Figura 4.1 ilustra a diferença entre a cópula gaussiana e a cópula de Clayton. As figuras representam simulações de Monte Carlo ${ }^{16}$ efetuadas pelo Mathematica 5.1, com $10^{4}$ realizações de duas variáveis estocásticas que possuem distribuições normais padronizadas. A estrututa de dependência das figuras à direita foi modelada pela cópula de Clayton com parâmetro $\theta=3,5$, que resultou em uma correlação igual $\rho=0,8$, e a cópula gaussiana foi modelada com o parâmetro $\rho=0,8$, i.e., ambas as distribuições bivariadas possuem a mesma dependência linear. Entretanto, como pode ser observado, a distribuição bivariada obtida pela cópula de Clayton é assimétrica, i.e., a estrutura da sua calda inferior é diferente da calda superior. A dependência da cauda inferior indica que existe uma maior probabilidade de saltos negativos ocorrerem em ambos os componentes conjuntamente. Esta característica não é observada na cópula gaussiana. A cópula de Clayton é representativa da classe de cópulas de Arquimedes, que pode ser definida pela seguinte proposição:

Proposição 4.1 Seja $\phi:[0 ; 1] \longrightarrow[0 ; \infty]$ uma função contínua estritamente crescente, então,

$$
C(u, v)=\phi^{-1}(\phi(u)+\phi(v))
$$

é uma cópula se e somente se $\phi$ for convexa e $\phi(0) \triangleq 1$.

Uma prova desta proposição e das generalizações multivariadas podem ser encontradas em Nelsen (1999) [cap.4, p.90 e p.121, respectivamente]. A função $\phi$ é denominada gerador da cópula de Arquimedes. $\phi(t)=\left(t^{-\phi}-1\right) / \phi$ produz a família Clayton e $\phi(t)=-\ln (t)$ produz a cópula independente $C_{\perp}$. Para a relação de outras famílias de cópulas pertencentes a classe de cópulas de Arquimedes consultar Nelsen (1999), p.94-97.

16 Método utilizado para simular as variáveis em conjunto é apresentado por Nelsen (1999), p.35. 

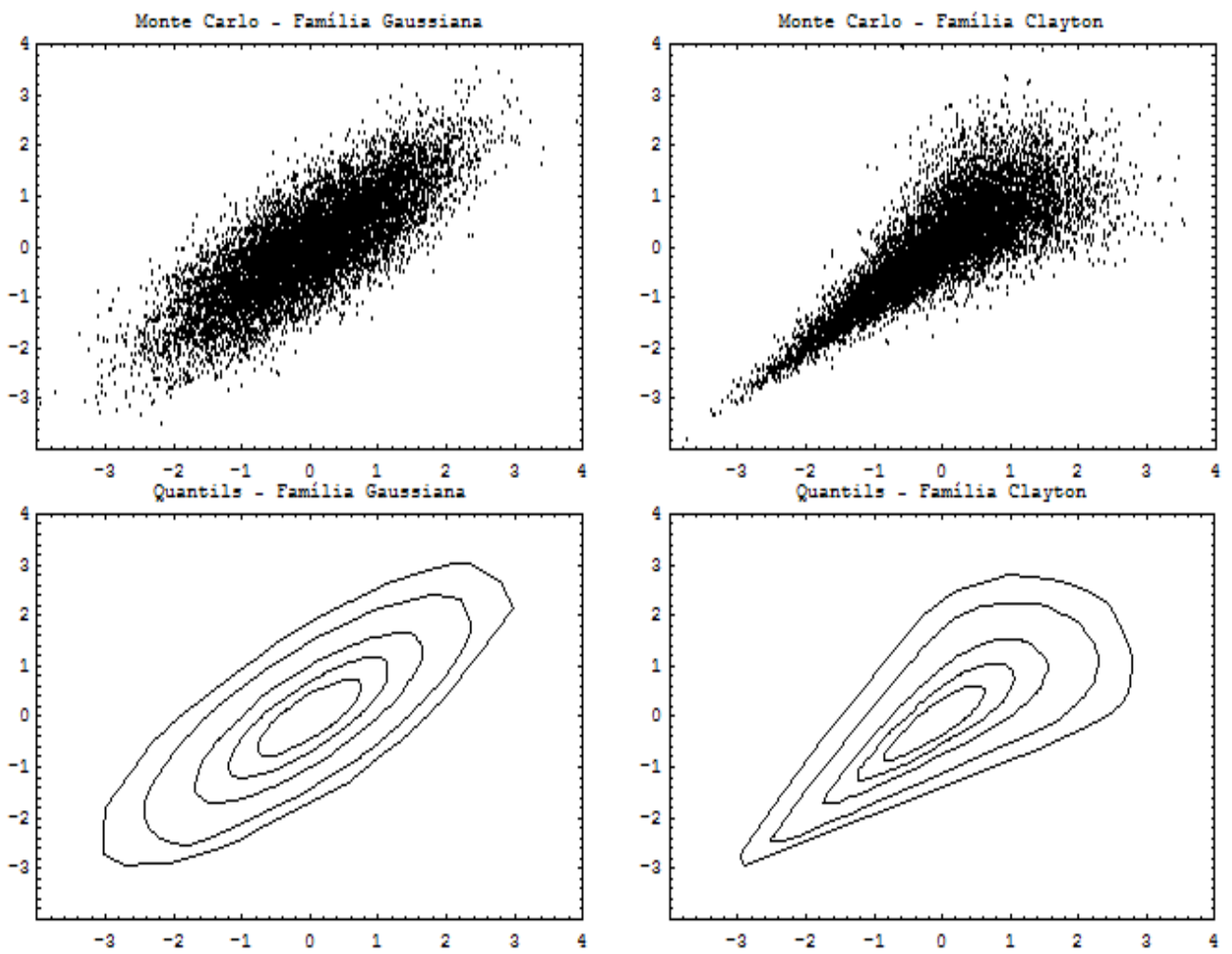

Figura 4.1. Simulação de Monte Carlo de duas marginais normais padronizadas com correlação igual a $\rho=0,8$. Direita superior: simulação da cópula de Clayton. Esquerda superior: simulação da cópula gaussiana. Direita e esquerda inferior quantis $99 \%, 95 \%$, $90 \%, 75 \%, 50 \%$ e $25 \%$ das cópulas acima. 


\subsubsection{Definições Estendidas aos Casos Multivariados}

Conforme Kallsen \& Tankov (2005), segue um sumário das definições estendidas aos casos multivariados:

Definição 4.4 F-Volume Seja $S_{1}, \ldots, S_{n}$ subconjuntos abertos à esquerda não vazios de $\overline{\mathbb{R}} e, F$ uma função real n-variada, tal que, $\mathcal{D}(F)=S_{1} \times \cdots \times S_{n}$ e, para $a, b \in \overline{\mathbb{R}}^{n}$, tal que $a \leq b$, i.e., $a_{k} \leq b_{k}$ para $k=1, \ldots, n$, seja $\left.\left.B=\right] a ; b\right]$ uma $n$-caixa, cujos vértices pertencem a $\mathcal{D}(F)$. Assim, o F-volume de B é definido como

$$
V_{F}(B)=\sum \operatorname{sgn}(c) F(c)
$$

sendo que a soma é realizada em todos os $2^{n}$ vértices $c \in \overline{\mathbb{R}}^{n}$ de $B$ e, $\operatorname{sgn}(c)$ é definido como

$$
\operatorname{sgn}(c) \triangleq\left\{\begin{aligned}
1, & \text { se } c_{k}=a_{k} \text { em quantidade par } \\
-1, & \text { se } c_{k}=a_{k} \text { em quantidade impar }
\end{aligned}\right.
$$

\section{Definição 4.5 função $n$-crescente, função aderente e marginais}

1. Uma função real $F$ de n-variada é denominada $n$-crescente se $V_{F}(B) \geq 0$ para todas as n-caixas $B$ cujos vértices pertencem à $\mathcal{D}(F)$.

2. Suponha que $\mathcal{D}(F)=S_{1} \times \cdots \times S_{n}$, sendo que cada $S_{k}$, $a_{k}=\inf S_{k}$, para $a_{k} \in \overline{\mathbb{R}}$. $F$ é uma função aderente se $F(t)=0, t \in \overline{\mathbb{R}}^{n}$, para todo $t \in \mathcal{D}(F)$ existir $t_{k}=a_{k}$ pelo menos para um $k$.

3. Se cada $S_{k}$ é um subconjunto não-vazio $e, b_{k}=\sup S_{k}$, para $b_{k} \in \overline{\mathbb{R}}$, então, as marginais (unidimensionais) de $F$ são funções $F_{k} \operatorname{com} \mathcal{D}\left(F_{k}\right)=S_{k}$ definida como $F_{k}(x)=F\left(b_{1}, \cdots, b_{k-1}, x, b_{k+1}, \cdots, b_{n}\right)$ para todo $x \in \mathcal{D}\left(F_{k}\right)$.

Definição 4.6 Cópula Uma cópula n-dimensional é uma função $C$ com $\mathcal{D}(C)=[0 ; 1]^{n}$, tal que:

1. Cé uma função aderente e n-crescente. 
2. C possui marginais $C_{k}, k=1, \ldots, n$, que satisfaz $C_{k}(u)=$ u para todo $u \in[0 ; 1]$.

Uma função de distribuição $n$-dimensional é uma função $F$ aderente e $n$-crescente com domínio $\mathcal{D}(F)=\overline{\mathbb{R}}^{n}$, tal que, $F(\infty, \cdots, \infty)=1$. Cópulas podem ser interpretadas como funções de distribuições com marginais uniformes.

Teorema 4.2 Sklar Seja $F$ uma função de distribuição n-dimensional com marginais $F_{1}, \ldots, F_{n}$. Assim, existe uma cópula n-dimensional $C$, tal que, para todo $x \in \overline{\mathbb{R}}^{n}$,

$$
F\left(x_{1}, \cdots, x_{n}\right)=C\left(F_{1}\left(x_{1}\right), \cdots, F_{n}\left(x_{n}\right)\right) .
$$

Se $F_{1}, \ldots, F_{n}$ são todas contínuas, então $C$ é única, caso contrário, $C$ será unicamente determinada em $\mathcal{D}(C)=\mathcal{I}\left(F_{1}\right) \times \ldots \times \mathcal{I}\left(F_{n}\right)$. De maneira inversa, se $C$ é uma $n$-cópula e $F_{1}, \ldots, F_{n}$ são funções de distribuições, então, a função $F$ definida pela equação (4.6) é um função de distribuição n-dimensional com marginais $F_{1}, \ldots, F_{n}$.

Conforme Kolev et al. (2005), uma conseqüência da Definição 4.6, é possibilidade de escrever a Cópula $n$-dimensional da seguinte maneira:

$$
C\left(F_{1}\left(x_{1}\right), \cdots, F_{n}\left(x_{n}\right)\right)=\prod_{i=1}^{n} F_{i}\left(x_{i}\right)+D\left(F_{1}\left(x_{1}\right), \cdots, F_{n}\left(x_{n}\right)\right),
$$

para $D\left(F_{1}\left(x_{1}\right), \cdots, F_{n}\left(x_{n}\right)\right) \in[0 ; 1]^{n}$ e $D\left(F_{1}\left(x_{1}\right), \cdots, F_{n}\left(x_{n}\right)\right)$ representa toda a informação da dependência de $\left\{X_{1}, \cdots, X_{n}\right\}$.

\subsection{Conceitos de Dependência para Processos de Lévy}

A distribuição de um processo de Lévy $X=\left\{X_{t}\right\}_{t \geq 0}$ é completamente determinada pela distribuição de $X_{t}$ para certo $t>0$. Assim, a estrutura de dependência de um processo de Lévy bidimensional $\left\{X_{t}, Y_{t}\right\}_{t \geq 0}$ pode ser parametrizada pela cópula $C_{t}$ de $X_{t}$ e $Y_{t}$ para certo $t>0$. Entretanto, esta abordagem apresenta as seguintes desvantagens:

1. Salvo no caso dos processos estáveis, a cópula $C_{t}$ pode depender de $t . C_{s}$ para certo $s \neq t$ não pode ser calculado a partir de $C_{t}$, pois ela também depende de suas marginais. 
2. Para uma distribuição infinitamente divisível de $X_{t}$ e $Y_{t}$, não é possível definir em quais circunstâncias a cópula $C_{t}$ representará uma distribuição bidimensional infinitamente divisível.

3. As distribuições dos componentes de um processo de Lévy multidimensional são usualmente especificadas pelas suas medidas de Lévy. Assim, levar em consideração apenas as distribuições para determinar a função de cópula não parece ser razoável.

O conceito tradicional de dependência de variáveis estocásticas, que significa que todas as características de uma distribuição são invariantes às transformações estritamente crescentes das marginais, não é adequado para caracterizar o conceito de dependência no contexto dos processos de Lévy. Segundo Cont \& Tankov (2004b) [cap.5, p.143], a propriedade de divisibilidade infinita de uma v.e. é destruída sob tranformações estritamentes crescentes desta v.e. Assim, é necessário redefinir o conceito de dependência, estabelecendo alguma outra forma de transfomação, que mantenha as propriedades da estrututa dinâmica de um processo de Lévy. O seguinte exemplo ilustra a observação:

Exemplo 4.2 - Dependência Completa Dinâmica de um Processo de Lévy Seja $X=\left\{X_{t}\right\}_{t \geq 0}$ um processo de Lévy que se move puramente por saltos e $Y=\left\{Y_{t}\right\}$ um processo de Lévy, construído por meio dos saltos de $\left\{X_{t}\right\}$, i.e., $Y_{t}=\sum_{s \leq t} \Delta X_{s}^{3}$. Pela perspectiva dinâmica, $X$ e $Y$ são completamente dependentes, pois a trajetória de um dos dois processos pode ser inteiramente reconstruída por meio da trajetória do outro. Entretanto, a cópula de $X_{t}$ e $Y_{t}$ não é completamente dependente, pois $Y_{t}$ não é uma função determinística de $X_{t}$, dado que a somatória depende da quantidade de saltos que ocorrem no processo, que é um v.e.

Este exemplo mostra que o importante conceito de dependência entre processos de Lévy é a dependência entre os saltos, que deve ser estudado por meio da medida de Lévy. Conhecimento da dependência dos saltos permite uma visão-micro de um processo de Lévy, que permite caracterizar sua estrutura dinâmica, que é muito importante para o gerenciamento de risco e outras aplicações financeiras. 
Como a estrutura de dependência da parte contínua de um processo de Lévy (MB) é inteiramente caracterizada pela matriz de covariância, é possível modelar a dependência dos saltos de maneira separada, pois pela decomposição de Lévy-Itô, o termo contínuo do processo de Lévy é independente do termo que produz os saltos. Assim, a partir deste ponto será considerado apenas processos de Lévy sem o componente gaussiano, i.e., $\Sigma=0$.

Pela proposição a seguir, é possível mostrar como a independência dos processos de Lévy e suas marginais podem ser representadas em termos de sua medida de Lévy.

Proposição 4.2 Marginais da Medida de Lévy Seja $\left\{X_{t}, Y_{t}\right\}_{t \geq 0}$ um processo de Lévy com tripé $(\Sigma, \nu, \gamma)$. Assim, $\left\{X_{t}\right\}_{t \geq 0}$ possui tripé característico $\left(\Sigma_{X}, \nu_{X}, \gamma_{X}\right)$, sendo que:

$$
\begin{aligned}
\Sigma_{X} & =\Sigma_{11} \\
\nu_{X}(B) & =\nu(B \times]-\infty ; \infty[), \forall B \in \mathcal{B}(\mathbb{R}) \\
\gamma_{X} & =\gamma_{1}+\int_{\mathbb{R}^{2}} x\left(1_{\left\{x^{2} \leq 1\right\}}-1_{\left\{x^{2}+y^{2} \leq 1\right\}}\right) \nu(d x \times d y) .
\end{aligned}
$$

O aspecto mais importante desta proposição é que as marginais de uma medida de Lévy podem ser calculadas da mesma maneira que as marginais de uma medida de probabilidade.

Prova O resultado segue diretamente do Teorema 3.1.

Proposição 4.3 Independência dos Processos de Lévy Seja $\left\{X_{t}, Y_{t}\right\}_{t \geq 0}$ um processo de Lévy com medida de Lévy $\nu$ sem o termo gaussiano. Seus componentes são independentes se e somente se o suporte (domínio) de $\nu$ está contido no conjunto $\{\{x, y\}: x y=0\}$, i.e., se e somente se os componentes não apresentam saltos no mesmo instante de tempo. Neste caso,

$$
\nu(B)=\nu_{X}\left(B_{X}\right)+\nu_{Y}\left(B_{Y}\right)
$$

sendo que $B_{X}=\{x:\{x, 0\} \in B\}$ e $B_{Y}=\{y:\{0, y\} \in B\}$, e $\nu_{X}$ e $\nu_{Y}$ são as medidas de Lévy de $\left\{X_{t}\right\}_{t \geq 0} e\left\{Y_{t}\right\}_{t \geq 0}$. 
Prova - Parte "somente se" Suponha que $\left\{X_{t}\right\}_{t \geq 0}$ e $\left\{Y_{t}\right\}_{t \geq 0}$ são independentes a possuem tripés característicos $\left(0, \nu_{X}, \gamma_{X}\right)$ e $\left(0, \nu_{Y}, \gamma_{Y}\right)$. Assim, utilizando a representação de Lévy-Khinchin para $X_{t}$ e $Y_{t}$ tem-se

$$
\begin{aligned}
\mathbb{E}\left[\exp \left\{i u X_{t}+i v Y_{t}\right\}\right]= & \mathbb{E}\left[\exp \left\{i u X_{t}\right\}\right] \mathbb{E}\left[\exp \left\{i v Y_{t}\right\}\right] \\
= & \exp \left\{t \left(i \gamma_{X} u+i \gamma_{Y} v\right.\right. \\
& +\int_{\mathbb{R}}\left(\exp \{i u x\}-1-i u x 1_{\{|x| \leq 1\}}\right) \nu_{X}(d x) \\
& \left.\left.+\int_{\mathbb{R}}\left(\exp \{i v y\}-1-i v y 1_{\{|y| \leq 1\}}\right) \nu_{Y}(d y)\right)\right\}(4.10)
\end{aligned}
$$

As integrais unidimensionais desta expressão podem ser reescritas na forma bidimensional:

$$
\begin{aligned}
& \int_{\mathbb{R}}\left(\exp \{i u x\}-1-i u x 1_{\{|x| \leq 1\}}\right) \nu_{X}(d x) \\
= & \int_{\mathbb{R}^{2}}\left(\exp \{i u x+i v y\}-1-(i u x+i v y) 1_{\left\{x^{2}+y^{2} \leq 1\right\}}\right) \\
& \times \tilde{\nu}_{X}(d x \times d y),
\end{aligned}
$$

sendo que $\tilde{\nu}_{X}(B)=\nu_{X}\left(B_{X}\right)$, para $\forall B \in \mathcal{B}(\mathbb{R})$. A igualdade apresentada na equação (4.11) é assegurada pois $\tilde{\nu}_{X}$ é suportado pelo conjunto $\{x:\{x, 0\}, x \in \mathbb{R}\}$, que significa que, no integrando, $y$ é igual a zero. Reescrevendo a segunda integral da equação (4.10) de maneira análoga, tem-se o resultado desejado.

Prova - Parte "se" Como a medida de Lévy é suportada pelo conjunto $\{\{x, y\}: x y=0\}$, ela pode ser representada pela equação (4.9), dado certas medidas positivas $\nu_{X}$ e $\nu_{Y}$. Pela Proposição (4.2) fica garantido que estas duas medidas coincidem com as medidas marginais de $\left\{X_{t}\right\}_{t \geq 0}$ e $\left\{Y_{t}\right\}_{t \geq 0}$. Para concluir aplicar a representação de Lévy-Kinchin ao processo $\left\{X_{t}, Y_{t}\right\}_{t \geq 0}$.

\subsection{Cópulas para Processos de Lévy com Saltos Positivos}

Conforme Cont \& Tankov (2004b) [cap.5, p.145], para parametrizar a dependência entre os saltos de processos de Lévy, a medida de Lévy executará o mesmo papel que a medida de probabilidade executa para as variáveis estocásticas. Assim, para modelar a dependência, é necessário criar cópulas para as medidas de Lévy. A principal dife- 
rença da cópula de uma v.e. para a cópula de uma medida de Lévy é que a medida de Lévy não é necessariamente finita, i.e., elas podem ter uma singularidade no zero que não é integrável. Desta maneira, cópulas de Lévy são definidas em intervalos infinitos, ao invés de intervalos em $[0 ; 1]^{2}$. Cópulas bidimensionais de Lévy para processos com saltos positivos são funções $C^{\mathcal{L}}:[0 ; \infty]^{2} \longrightarrow[0 ; \infty]$ e, de uma forma geral, cópulas de Lévy são funções $C^{\mathcal{L}}: \overline{\mathbb{R}}^{2} \longrightarrow \overline{\mathbb{R}}$.

Para o caso de processos de Lévy com saltos positivos, o papel da função de distribuição é exercido pela integral caudal. Inicialmente, não será imposto nenhuma condição de integrabilidade or continuidade.

Definição 4.7 Uma integral caudal d-dimensional é uma função $U:[0 ; \infty]^{d} \longrightarrow$ $[0 ; \infty]$, tal que:

1. $(-1)^{d} U$ é uma função d-crescente;

2. U é igual a zero se algum de seus argumentos for igual a $\infty$;

3. U é finito em todos os lugares exceto no zero e, $U(0, \ldots, 0)=\infty$.

É possível interpretar $U$, como a integral caudal de uma medida de Lévy:

$$
U\left(x_{1}, \cdots, x_{d}\right)=\nu\left(\left[x_{1} ; \infty\left[\times \cdots \times\left[x_{d} ; \infty[), x_{1}, \ldots, x_{d} \in \mathbb{R}_{+}^{d} \backslash\{0\} .\right.\right.\right.\right.
$$

As marginais de uma integral caudal são definidas como:

$$
U\left(0, \cdots, 0, x_{k}, 0, \cdots, 0\right)=U_{k}\left(x_{k}\right) .
$$

Definição 4.8 Cópula de Lévy para Processos com Saltos Positivos Uma cópula de Lévy bidimensional para processos de Lévy com saltos positivos, ou abreviadamente, cópula de Lévy positiva, é um função 2-crescente e aderente $C^{\mathcal{L}}:[0 ; \infty]^{2} \longrightarrow[0 ; \infty]$ com marginais uniformes, i.e., $C^{\mathcal{L}}(x, \infty)=C^{\mathcal{L}}(\infty, x)=x .{ }^{17}$

O seguinte teorema é uma reformulação do Teorema de Sklar para integrais de caudas e cópulas de Lévy. Ela mostra que as cópulas de Lévy estão relacionadas aos in-

17 No caso geral, cópulas para os processos de Lévy serão definidas como funções $F: \overline{\mathbb{R}}^{2} \longrightarrow \overline{\mathbb{R}}$. Apesar disto, cópulas de Lévy positivas podem ser estendidas para o caso geral, ao estabelecer que $F(x, y)=0$ se $x<0$ ou $y<0$. 
tegrais caudais multidimensionais e suas respectivas marginais de maneira equivalente como as cópulas de variáveis estocásticas relacionam a função de distribuição com suas marginais.

Teorema 4.3 Seja U uma integral caudal bidimensional com marginais $U_{1}$ e $U_{2}$. Assim, existe uma cópula de Lévy positiva, tal que,

$$
U\left(x_{1}, x_{2}\right)=C^{\mathcal{L}}\left(U_{1}\left(x_{1}\right), U_{2}\left(x_{2}\right)\right) .
$$

Se $U_{1}$ e $U_{2}$ são contínuas, esta cópula de Lévy é única. Caso contrário, ela será unicamente determinada em $\mathcal{D}\left(C^{\mathcal{L}}\right)=\mathcal{I}\left(U_{1}\right) \times \mathcal{I}\left(U_{2}\right)$, i.e., o produto cartesiano das imagens das integrais caudais unidimensionais. De maneira inversa, se $C^{\mathcal{L}}$ é uma cópula de Lévy positiva e $U_{1}$ e $U_{2}$ são integrais caudais unidimensionais, então, a equação (4.13) define uma integral caudal bidimensional.

Prova Apenas para simplificar o raciocínio, suponha que $U_{1}$ e $U_{2}$ são contínuos. A prova para o caso geral pode ser obtida por meio do Teorema de Sklar [Kallsen \& Tankov (2005)]. Escolha $U_{1}^{-1}$ e $U_{2}^{-1}$, tal que, $U_{1}^{-1}(0)=U_{2}^{-1}(0)=\infty$, $U_{1}^{-1}(\infty)=U_{2}^{-1}(\infty)=0$ e,

$$
C^{\mathcal{L}}\left(y_{1}, y_{2}\right)=U\left(U_{1}^{-1}\left(y_{1}\right), U_{2}^{-1}\left(y_{2}\right)\right)
$$

É possível verificar que $C^{\mathcal{L}}$ é uma cópula de Lévy e que satisfaz a equação (4.13). Suponha que exista uma outra cópula de Lévy $\tilde{C}^{\mathcal{L}}$. Assim, se para cada $x_{1} \in[0 ; \infty]$ e $x_{2} \in[0 ; \infty], C^{\mathcal{L}}\left(U_{1}\left(x_{1}\right), U_{2}\left(x_{2}\right)\right)=\tilde{C}^{\mathcal{L}}\left(U_{1}\left(x_{1}\right), U_{2}\left(x_{2}\right)\right)$. Dado que $U_{1}$ e $U_{2}$ são contínuas, tem-se que para todo $y_{1} \in[0 ; \infty]$ e $y_{2} \in[0 ; \infty], C^{\mathcal{L}}\left(y_{1}, y_{2}\right)=\tilde{C}^{\mathcal{L}}\left(y_{1}, y_{2}\right)$. A observação inversa pode ser obtida de maneira equivalente. 


\subsubsection{Integral Caudal e Medidas de Lévy}

Para toda medida de Lévy $\nu$ em $[0 ; \infty$ [, é possível definir sua integral caudal da seguinte maneira:

$$
\begin{aligned}
U\left(x_{1}, x_{2}\right) & =0 \text { se } x_{1}=\infty \text { ou } x_{2}=\infty \\
U\left(x_{1}, x_{2}\right) & =\nu\left(\left[x_{1} ; \infty\left[,\left[x_{2} ; \infty[) \text { para }\left\{x_{1}, x_{2}\right\} \in \mathbb{R}_{+}^{2} \backslash\{0\}\right.\right.\right.\right. \\
U(0,0) & =\infty .
\end{aligned}
$$

Dado as mesmas propriedades sobre continuidade apresentadas na Definição 4.2, uma integral caudal bidimensional define uma medida positiva $\nu$ em $\mathbb{R}_{+}^{2} \backslash\{0\}$. Entretanto, para que seja uma medida de Lévy, $\nu$ deve satisfazer a condição de integrabilidade apresentada no Proposição 2.7:

$$
\int_{[0 ; \infty]^{2}}\left(|x|^{2} \wedge 1\right) \nu(d x)<\infty=\int_{[0 ; \infty]^{2}}\left(|x|^{2} \wedge 1\right) U(d x)<\infty
$$

sendo que $U$ é uma medida de Lebesgue, que existe porque $U$ é uma função crescente. O seguinte lema mostra quando esta condição de integrabilidade é satisfeita.

Lema 4.2 Seja U uma integral caudal bidimensional com marginais $U_{1}$ e $U_{2}$. $U$ define uma medida de Lévy em $\mathbb{R}_{+}^{2} \backslash\{0\}$, i.e., a condição de integrabilidade apresentada na equação (4.15) é satisfeita se e somente se as marginais de U correspondem a medidas de Lévy em $\mathbb{R}_{+}$, i.e., para $k \in\{1,2\}$,

$$
\int_{0}^{1} x^{2} U_{k}(d x)<\infty
$$


Prova A equivalência segue da seguinte forma:

$$
\begin{aligned}
\int_{[0 ; 1]^{2}}|x|^{2} U(d x) & =\int_{[0 ; 1]^{2}} \sum_{i=1}^{2} x_{i}^{2} U(d x)=\sum_{i=1}^{2} \int_{[0 ; 1]^{2}} x_{i}^{2} U(d x) \\
& =\sum_{i=1}^{2}\left(\int_{[0 ; 1] \times[0 ; \infty]} x_{i}^{2} d U(x)-\int_{[0 ; 1] \times[1 ; \infty]} x_{i}^{2} U(d x)\right) \\
& =\sum_{i=1}^{2}\left(\int_{[0 ; 1]} x_{i}^{2} U\left(d x_{i} \times[0 ; \infty]\right)-C_{i}\right) \\
& =\sum_{i=1}^{2}\left(\int_{[0 ; 1]} x_{i}^{2} U_{i}\left(d x_{i}\right)-C_{i}\right)
\end{aligned}
$$

sendo que a constante, para $i \in\{1,2\}$ :

$$
C_{i}=\int_{[0 ; 1] \times[1 ; \infty]} x_{i}^{2} U(d x) \leq \int_{[0 ; 1] \times[1 ; \infty]} d U(x)
$$

são constantes. Desta maneira, ao utilizar a Definição 4.8, tem-se que

$$
\sum_{i=1}^{2} \int_{[0 ; 1]} x_{i}^{2} U_{i}\left(d x_{i}\right)=\int_{[0 ; 1]^{2}}|x|^{2} U(d x)+\sum_{i=1}^{2} C_{i},
$$

Como é possível verificar que $\int_{[1 ; \infty]^{2}} d U(x)<\infty$ e, se $\int_{0}^{1} x_{i}^{2} U_{i}\left(d x_{i}\right)<\infty$ para $i \in$ $\{1,2\}$, então, a condição de integrabilidade

$$
\int_{[0 ; \infty]^{2}}\left(|x|^{2} \wedge 1\right) U(d x)
$$

é finita, e $U(x)$ representa uma medida de Lévy.

Teorema 4.4 Seja $\left\{X_{t}, Y_{t}\right\}_{t \geq 0}$ um processo de Lévy bidimensional com saltos positivos, integral caudal $U$ e marginais da integral caudal $U_{1}$ e $U_{2}$. Assim, existe uma cópula de Lévy bidimensional $C^{\mathcal{L}}$ que caracteriza a estrutura de dependência de $\left\{X_{t}, Y_{t}\right\}_{t \geq 0}$, i.e., para todo $x_{1}, x_{2} \in[0 ; \infty]$,

$$
U\left(x_{1}, x_{2}\right)=C^{\mathcal{L}}\left(U_{1}\left(x_{1}\right), U_{2}\left(x_{2}\right)\right) .
$$

Se $U_{1}$ e $U_{2}$ forem contínuas, esta cópula de Lévy é única. Caso contrário, ela será unicamente determinada em $\mathcal{D}\left(C^{\mathcal{L}}\right)=\mathcal{I}\left(U_{1}\right) \times \mathcal{I}\left(U_{2}\right)$. De maneira inversa, seja $\left\{X_{t}\right\}_{t \geq 0}$ $e\left\{Y_{t}\right\}_{t \geq 0}$ dois processos de Lévy unidimensionais com saltos positivos que possuem integrais caudais $U_{1}$ e $U_{2}$ e, $C^{\mathcal{L}}$ é uma cópula positiva de Lévy bidimensional. Assim, existe um processo de Lévy com cópula de Lévy $C^{\mathcal{L}}$ com integrais caudais marginais $U_{1}$ e $U_{2}$, sendo que a integral caudal será dada pela equação (4.16). 
Este resultado é uma conseqüência direta do Teorema 4.3 e do Lema 4.2. A primeira parte deste teorema dispõe que todos os tipos de dependência existentes entre processos de Lévy, incluindo dependência completa e independência, podem ser representadas por cópulas de Lévy e, a segunda parte do teorema, mostra que é possível construir modelos baseados em processos de Lévy multivariados ao especificar a estrutura de dependência dos saltos separadamente das distribuições unidimensionais dos componentes do modelo. A distribuição dos componentes podem apresentar estruturas bastante diferentes e, em particular, é possível copular processos compostos de Poisson com os de atividades infinitas.

Conforme Cont \& Tankov (2004b), [cap.5, p. 148], quando a dependência é especificada por meio de uma cópula de Lévy e, a cópula e as integrais caudais marginais são suficientemente suavisadas, a medida de Lévy pode ser calculada pela seguinte diferenciação:

$$
\nu\left(x_{1}, x_{2}\right)=\left.\frac{\partial^{2} C^{\mathcal{L}}\left(y_{1}, y_{2}\right)}{\partial y_{1} \partial y_{2}}\right|_{y_{1}=U_{1}\left(x_{1}\right), y_{1}=U_{2}\left(x_{2}\right)} \nu_{1}\left(x_{1}\right) \nu_{2}\left(x_{2}\right)
$$

\subsubsection{Exemplos de Cópulas de Lévy Positivas}

É possível calcular a cópula de Lévy que corresponde as várias estruturas de dependência básicas.

Exemplo 4.3 - Independência Seja $\left\{X_{t}, Y_{t}\right\}_{t \geq 0}$ um processo de Lévy com componentes independentes. Pela Proposição 4.3, a medida de Lévy deste processo é dada por

$$
\begin{aligned}
\nu(B) & =\nu_{1}\left(B_{X}\right)+\nu_{2}\left(B_{Y}\right) \\
\nu\left(\left[x_{1} ; \infty\right] \times\left[x_{2} ; \infty\right]\right) & =\nu_{1}\left(\left[x_{1} ; \infty\right]\right)+\nu_{2}\left(\left[x_{2} ; \infty\right]\right) \\
& =\nu\left(\left[x_{1} ; \infty\right] \times[0 ; \infty]\right) 1_{\left\{x_{2}=0\right\}}+\nu\left([0 ; \infty] \times\left[x_{2} ; \infty\right]\right) 1_{\left\{x_{1}=0\right\}} \\
U\left(x_{1}, x_{2}\right) & =U\left(x_{1}, 0\right) 1_{\left\{x_{2}=0\right\}}+U\left(0, x_{2}\right) 1_{\left\{x_{1}=0\right\}} \\
& =U_{1}\left(x_{1}\right) 1_{\left\{x_{2}=0\right\}}+U_{2}\left(x_{2}\right) 1_{\left\{x_{1}=0\right\}} .
\end{aligned}
$$


Por meio da equação (4.14) pode-se calcular a cópula de Lévy de processos independentes:

$$
\begin{aligned}
C_{\perp}^{\mathcal{L}}\left(y_{1}, y_{2}\right) & =U\left(U_{1}^{-1}\left(y_{1}\right), U_{2}^{-1}\left(y_{2}\right)\right) \\
& =U_{1}\left(U_{1}^{-1}\left(y_{1}\right)\right) 1_{\left\{U_{2}^{-1}\left(y_{2}\right)=0\right\}}+U_{2}\left(U_{2}^{-1}\left(y_{2}\right)\right) 1_{\left\{U_{1}^{-1}\left(y_{1}\right)=0\right\}} \\
& =y_{1} 1_{\left\{y_{2}=\infty\right\}}+y_{2} 1_{\{y 1=\infty\}} .
\end{aligned}
$$

Se $U_{1}$ e $U_{2}$ não forem contínuos, a equação (4.17) será apenas uma das possibilidades de cópulas de Lévy para o processo bidimensional $\left\{X_{t}, Y_{t}\right\}_{t \geq 0}$.

Para discutir a dependência completa dos saltos ou comonotonicidade dos componentes de um processo de Lévy com saltos positivos, é necessário definir a idéia de um conjunto crescente.

Definição 4.9 Um subconjunto $S \subset \overline{\mathbb{R}}^{2}$ é denominado crescente se para todo dois vetores $\left\{v_{1}, v_{2}\right\} \in S$ e $\left\{u_{1}, u_{2}\right\} \in S$, tanto $v_{k}<u_{k}$ ou $v_{k}>u_{k}$ para $\forall k$.

Um elemento de um conjunto crescente é completamente determinado por apenas uma coordenada, o que motiva a seguinte definição de dependência dos saltos:

Definição 4.10 Seja $X=\left\{X_{t}^{1}, X_{t}^{2}\right\}_{t \geq 0}$ um processo de Lévy com saltos positivos. Seus saltos são completamente dependentes ou comonotônicos se existir um subconjunto crescente $S \in] 0 ; \infty\left[{ }^{2}\right.$, tal que, para todo salto $\Delta X \in S$.

Se os saltos de dois processos de Lévy que se movem puramente por saltos forem completamente dependentes, a trajetória de um deles pode ser reconstruída por meio do conhecimento da trajetória do outro.

Proposição 4.4 Dependência Completa Seja $X=\left\{X_{t}^{1}, X_{t}^{2}\right\}_{t \geq 0}$ um processo de Lévy com saltos positivos. Se os seus saltos forem completamente dependentes, então, a (possivel) cópula de Lévy de X é uma cópula de Lévy completamente dependente, que é definida por:

$$
C_{\|}^{\mathcal{L}}\left(x_{1}, x_{2}\right)=\min \left(x_{1}, x_{2}\right) .
$$


De maneira inversa, se uma cópula de Lévy de $X$ é dado por $C_{\|}^{\mathcal{L}}$ e, as integrais caudais de seus componentes forem contínuas, então, os saltos de $X$ são completamente dependentes.

Prova Os saltos de $X$ são completamente dependentes se e somente se existir um subconjunto crescente $S \in] 0 ; \infty\left[^{2}\right.$, tal que, a medida de Lévy $\nu$ está concentrada em $S$. Desta maneira, para todo $x=\left\{x_{1}, x_{2}\right\}$ e $y=\left\{y_{1}, y_{2}\right\}$,

$$
U(x)=\nu(\{y \geq x\} \cap S)=\int_{\{y \geq x\} \cap S} \nu(d y) .
$$

Por meio desta igualdade é possível obter as desigualdades para as marginais:

$$
U_{k}\left(x_{k}\right)=\nu\left(\left\{y_{k} \geq x_{k}\right\} \cap S\right)=\int_{\left\{y_{k} \geq x_{k}\right\} \cap S} \nu(d y), k \in\{1,2\} .
$$

Conforme Kallsen \& Tankov (2005), é possível verificar que $U(x) \leq U_{k}\left(x_{k}\right)$, para qualquer $k \mathrm{e}$,

$$
\left\{y \in S: y_{k} \geq x_{k}\right\} \cap S=\{y \in S: y \geq x\} \cap S
$$

para certo $k$, o que implica em

$$
U(x)=\min \left(U_{1}\left(x_{1}\right), U_{1}\left(x_{2}\right)\right) .
$$

Por outro lado, quando a integral caudal de $X$ apresenta a forma da equação (4.18) significa que a medida de Lévy está concentrada no conjunto $\left\{\left\{x_{1}, x_{2}\right\}: U_{1}\left(x_{1}\right)=U_{2}\left(x_{2}\right)\right\}$. Se as integrais caudais $U_{1}$ e $U_{2}$ forem contínuas, então este conjunto é crescente.

Exemplo 4.4 - Dependência de Processos Estáveis Cópulas de Lévy de processos estáveis são funções homogêneas de ordem 1, i.e.,

$$
\forall c>0, C^{\mathcal{L}}\left(c x_{1}, c x_{2}\right)=c C^{\mathcal{L}}\left(x_{1}, x_{2}\right) .
$$

Pela fórmula (2.32), a medida de Lévy de processos estáveis satisfaz

$$
\nu(B)=c^{\alpha} \nu(c B), \forall c>0, \forall B \in \mathcal{B}\left(\mathbb{R}^{2}\right) .
$$

Isto significa que a integral caudal satisfaz

$$
U\left(c x_{1}, c x_{2}\right)=c^{-\alpha} U\left(x_{1}, x_{2}\right) .
$$


Ao substituir as marginais $\alpha$-estáveis nesta fórmula, é possível concluir que a cópula de Lévy de um processo estável é homogênea de ordem 1 . A estrutura de dependência de dois processos estáveis de Lévy pode ser especificada pela parte esférica da medida de Lévy de um processo bidimensional [Proposição 2.15]. Neste caso, o processo de Lévy bidimensional também será estável [Cont \& Tankov (2004b), cap.5, p.150]. Cópulas de Lévy também permitem estabelecer uma grande variedade de estruturas de dependência, pois ao utilizar cópulas de Lévy é possível construir processos de Lévy que possuem marginais $\alpha$-estáveis, mas que não é $\alpha$-estável. Para fazer isto é suficiente utilizar uma cópula de Lévy que não seja homogênea, e.g.,

$$
C^{\mathcal{L}}(u, v)=\ln \left(\frac{1-\exp \{-u-v\}}{\exp \{-u\}+\exp \{-v\}-2 \exp \{-u-v\}}\right) .
$$

Este exemplo é uma cópula de Lévy da família Archimedes com gerador $\phi(x)=\frac{\exp \{-x\}}{1-\exp \{-x\}}$ [Proposição 4.6].

\subsubsection{Dependência de Processos Compostos de Poisson}

A dependência de um processo composto de Poisson pode ser descrita por meio de cópulas ordinárias. Assim, uma conseqüência da decomposição de Lévy-Itô é que os processos compostos de Poisson bidimensional $\left\{X_{t}, Y_{t}\right\}_{t \geq 0}$ sempre podem ser divididos em partes dependentes e independentes [Seção 4.2], i.e.,

$$
\begin{aligned}
X_{t} & =X_{t}^{\perp}+X_{t}^{\|}, \\
Y_{t} & =Y_{t}^{\perp}+Y_{t}^{\|}
\end{aligned}
$$

sendo que, $\left\{X_{t}^{\perp}\right\}_{t \geq 0}$ e $\left\{Y_{t}^{\perp}\right\}_{t \geq 0}$ são independentes (parte independente) e, $\left\{X_{t}^{\|}\right\}_{t \geq 0} \mathrm{e}$ $\left\{Y_{t}^{\|}\right\}_{t \geq 0}$ são dependentes, possuem a mesma intensidade e saltam no mesmo instante de tempo (parte dependente). As medidas de Lévy dos componentes estão relacionadas à medida de Lévy $\nu$ de $\left\{X_{t}, Y_{t}\right\}_{t \geq 0}$ :

$$
\begin{aligned}
\nu_{X}^{\perp}\left(B_{X}\right) & =\nu\left(B_{X} \times\{0\}\right), \forall B_{X} \in \mathcal{B}\left(\mathbb{R}_{+}\right), \\
\nu_{Y}^{\perp}\left(B_{Y}\right) & =\nu\left(\{0\} \times B_{Y}\right), \forall B_{Y} \in \mathcal{B}\left(\mathbb{R}_{+}\right), \\
\nu^{\|}(B) & =\nu(B)-\nu\left(B_{X} \times\{0\}\right)-\nu\left(\{0\} \times B_{Y}\right), \forall B \in \mathcal{B}\left(\mathbb{R}_{+}^{2}\right) .
\end{aligned}
$$

Os conjuntos $B_{X}$ e $B_{Y}$ são definidos da maneira apresentada na Proposição (4.3).

Para definir o modelo completamente é necessário especificar: 
1. A intensidade e a distribuição do tamanho do salto de $X^{\perp}$ e $Y^{\perp}$;

2. A intensidade das inovações em comum;

3. A distribuição do tamanho dos saltos de $X^{\|}$e $Y^{\|}$; e

4. A cópula para as distribuições dependentes $X^{\|}$e $Y^{\|}$.

Desta maneira, esta abordagem requer a especificação de diferentes quantidades, sendo que algumas são difíceis de ser especificadas. Adicionalmente, ela não permite separar completamente a dependência das marginais, pois as intensidades e as distribuições dos componentes são determinadas tanto pela estrutura de dependência quanto pelas marginais. Assim, é conveniente utilizar a abordagem da cópula de Lévy. Esta outra abordagem requer a especificação apenas de:

1. Das marginais por meio das intensidades e distribuição dos saltos de $X$ e $Y$ (que é observado); e

2. A estrutura de dependência via cópula de Lévy do processo bidimensional.

Todas as outras quantidades são calculadas por meio destas, sendo que é necessário apenas supor que a cópula é contínua. Seja $C^{\mathcal{L}}$ a cópula de Lévy e $U_{X}$ e $U_{Y}$ integrais caudais de $X$ e $Y$. Assim, $X$ e $Y$ são:

$$
\lambda_{X}=\lim _{x \downarrow 0} U_{X}(x) \text { e } \lambda_{X}=\lim _{y \downarrow 0} U_{Y}(x) .
$$

Não é possível simplesmente escrever $\lambda_{X}=U_{X}(0)$, pois pela Definição 4.7, $U_{X}(0)=$ $\infty$ para todos os processos, incluindo os processos compostos de Poisson. A integral caudal de um processo bidimensional $\left\{X_{t}, Y_{t}\right\}_{t \geq 0}$ é $U(x, y)=C^{\mathcal{L}}\left(U_{X}(x), U_{Y}(y)\right)$. A intensidade é igual a medida de Lévy definida em $\mathbb{R}_{+}^{2} \backslash\{0\}$, o que significa que

$$
\begin{aligned}
\lambda & =\lim _{x, y \downarrow 0}\left(U_{X}(x)+U_{Y}(y)-U(x, y)\right) \\
& =\lim _{x, y \downarrow 0}\left(U_{X}(x)+U_{Y}(y)-C^{\mathcal{L}}\left(U_{X}(x), U_{Y}(y)\right)\right) \\
& =\lambda_{X}+\lambda_{Y}-C^{\mathcal{L}}\left(\lambda_{X}, \lambda_{Y}\right) .
\end{aligned}
$$

Como toda cópula de Lévy positiva satisfaz $0 \leq C^{\mathcal{L}}(x, y) \leq \min (x, y)$, a intensidade está contida entre $\max \left(\lambda_{X}, \lambda_{Y}\right)$ (dependência completa) e $\lambda_{X}+\lambda_{Y}$ (independência). 
A intensidade dos saltos comuns de um processo de Poisson (intensidade de $X_{t}^{\|}$e $Y_{t}^{\|}$) é igual a $C^{\mathcal{L}}\left(\lambda_{X}, \lambda_{Y}\right)$. As integrais caudais dos componentes independentes são

$$
\begin{aligned}
U_{X}^{\perp}(x) & =\nu\left(\left[x ; \infty[\times\{0\})=U(x, 0)-\lim _{y \downarrow 0} U(x, y)\right.\right. \\
& =U_{X}(x)-C^{\mathcal{L}}\left(U(x), \lambda_{Y}\right) \mathrm{e} \\
U_{Y}^{\perp}(y) & =U_{Y}(x)-C^{\mathcal{L}}\left(\lambda_{X}, U(y)\right),
\end{aligned}
$$

para $x, y \in] 0 ; \infty\left[\right.$. Finalmente, a integral caudal de $\left\{X_{t}^{\|}, Y_{t}^{\|}\right\}_{t \geq 0}$ é

$$
U^{\|}(x, y)=\lim _{x^{\prime} \downarrow x, y^{\prime} \downarrow y} U\left(x^{\prime}, y^{\prime}\right)=C^{\mathcal{L}}\left(\min \left(U_{X}(x), \lambda_{X}\right), \min \left(U_{Y}(y), \lambda_{Y}\right)\right)
$$

para $\{x, y\} \in\left[0 ; \infty\left[^{2} \backslash\{0\}\right.\right.$, e a função de sobrevivência ${ }^{18}$ da distribuição dos saltos possui a seguinte forma:

$$
H(x, y)=\frac{C^{\mathcal{L}}\left(\min \left(U_{X}(x), \lambda_{X}\right), \min \left(U_{Y}(y), \lambda_{Y}\right)\right)}{C^{\mathcal{L}}\left(\lambda_{X}, \lambda_{Y}\right)} .
$$

\subsubsection{Construção de Cópulas Positivas de Lévy}

Cópulas de Lévy podem ser calculadas por meio de processos de Lévy multidimensionais ao se utilizar a fórmula (4.14). Entretanto, este método não é muito útil, pois não existem muitos modelos multidimensionais, como já havia sido observado. Assim, é necessário desenvolver outras abordagens:

Proposição 4.5 Seja C uma 2-cópula (ordinária) e $f(x)$ uma função convexa crescente definida como $f:[0 ; 1] \longrightarrow[0 ; \infty]$. Assim,

$$
C^{\mathcal{L}}(x, y)=f\left(C\left(f^{-1}(x), f^{-1}(y)\right)\right)
$$

define uma cópula de Lévy positiva bidimensional.

Prova $\mathrm{O}$ fato de $C^{\mathcal{L}}$ ser uma função 2-crescente provém das Propriedades 1 e 2 do Lema 4.1. Desta maneira, é possível verificar que $C^{\mathcal{L}}$ é aderente e as propriedades das marginais são satisfeitas.

18 A função de sobrevivência $H_{X}(x)$ de um v.e. $X$ é definida como $H_{X}(x)=\mathbb{P}[X>x]$ e a função de sobrevivência conjunta de duas variáveis estocásticas $X$ e $Y$ é $H(x, y)=\mathbb{P}[X>x, Y>y]$. 
Existem diversão funções $f:[0 ; 1] \longrightarrow[0 ; \infty]$ com derivadas positivas de todas as ordens que podem ser utilizadas nesta proposição, e.g., $f(x)=\frac{x}{1-x}$. Por analogia, por meio das cópulas de Arquimedes é possível construir as cópulas de Lévy de Arquimedes.

Proposição 4.6 Seja $\phi(u)$ uma função convexa estritamente descrescente $\phi$ : $[0 ; \infty] \longrightarrow[0 ; \infty]$, tal que, $\phi(0)=\infty$ e $\phi(\infty)=0$. Assim,

$$
C^{\mathcal{L}}(x, y)=\phi^{-1}(\phi(x)+\phi(y))
$$

define uma cópula de Lévy positiva bidimensional.

Prova Esta prova é novamente o resultado das Propriedades 1 e 2 do Lema 4.1, dado que $\phi^{-1}(-u)$ é crescente e convexa.

Exemplo 4.5 Para $\phi(u)=u^{-\theta} \operatorname{com} \theta>0$, obtém-se a seguinte família paramétrica de cópulas de Lévy:

$$
C_{\theta}^{\mathcal{L}}(u, v)=\left(u^{-\theta}+v^{-\theta}\right)^{-1 / \theta}
$$

relembra a família de cópulas de Clayton [equação 4.5]. Esta família apresenta os casos limites de completa dependência $(\theta \longrightarrow \infty)$ e independência $(\theta \longrightarrow 0)$.

\subsubsection{Interpretação Probabilística}

Diferentemente das cópulas ordinárias, as cópulas de Lévy não são funções de distribuições, mas as suas derivadas possuem uma interpretação probabilística. Cont \& Tankov (2004b), [cap.5, p.154], provam o seguinte lema:

Lema 4.3 Seja $C^{\mathcal{L}}$ uma cópula de Lévy bidimensional. Assim, para todo $x \in$ $[0 ; \infty]$, a função

$$
C_{x}^{\mathcal{L}}(y)=\frac{\partial}{\partial x} C^{\mathcal{L}}(x, y)
$$

existe e é contínua para todo $y \in[0 ; \infty]$. Desta maneira, $C_{x}^{\mathcal{L}}(y)$ é uma função de distribuição de uma v.e. positiva, i.e., $C_{x}^{\mathcal{L}}(y)$ é uma função crescente e satisfaz $C_{x}^{\mathcal{L}}(0)=0$ $e C_{x}^{\mathcal{L}}(\infty)=1$. 
Assim o seguinte teorema, mostra que $C^{\mathcal{L}}$ determina uma distribuição de saltos (transformados) do segundo componente, condicionado ao tamanho do salto do primeiro componente. Esta propriedade pode ser útil para simular processos.

Teorema 4.5 Seja $\left\{X_{t}, Y_{t}\right\}_{t \geq 0}$ um processo de Lévy bidimensional com saltos positivos, possuindo integrais caudais marginais $U_{1}$ e $U_{2}$ e cópula de Lévy igual a $C^{\mathcal{L}}$. Seja $\Delta X_{t}$ e $\Delta Y_{t}$ o tamanho dos saltos dos dois componentes do processo no instante de tempo t. Assim, se $U_{1}$ possuir uma densidade não-negativa em $x, C_{U_{1}(x)}^{\mathcal{L}}$ é a função de distribuição de $U_{2}\left(\Delta Y_{t}\right)$ condicionada em $\Delta X_{t}=x$ :

$$
C_{U_{1}(x)}^{\mathcal{L}}(y)=\mathbb{P}\left[U_{2}\left(\Delta Y_{t}\right) \mid \Delta X_{t}=x\right] .
$$

\subsubsection{Definições Estendidas aos Casos Multivariados}

Segue sumário das definições estendidas aos casos multivariados:

Definição 4.11 Cópula de Lévy Positiva Uma cópula de Lévy positiva $n$-dimensional é uma função n-crescente e aderente $C^{\mathcal{L}}:[0 ; \infty]^{n} \longrightarrow[0 ; \infty]$ com marginais $C_{k}^{\mathcal{L}}, k=1,2, \ldots, n$, que satisfazem $C_{k}^{\mathcal{L}}(u)=$ u para todo $u \in[0 ; \infty]$.

Teorema 4.6 Seja U uma integral caudal de um processo de Lévy com saltos positivos n-dimensional e, seja $U_{1}, \ldots, U_{k}$ as integrais caudais de seus componentes. Assim, existe uma cópula de Lévy positiva n-dimensional $C^{\mathcal{L}}$, tal que, todos os vetores $\left\{x_{1}, \cdots, x_{n}\right\}$ em $\mathbb{R}_{+}^{n}$,

$$
U\left(x_{1}, \cdots, x_{n}\right)=C^{\mathcal{L}}\left(U_{1}\left(x_{1}\right), \cdots, U_{n}\left(x_{n}\right)\right) .
$$

Se $U_{1}, \ldots, U_{n}$ são contínuas, então, $C^{\mathcal{L}}$ é unica, caso contrário, é unicamente determinada em $\mathcal{D}\left(C^{\mathcal{L}}\right)=\mathcal{I}\left(U_{1}\right) \times \cdots \times \mathcal{I}\left(U_{n}\right)$. De maneira inversa, se $C^{\mathcal{L}}$ é uma cópula de Lévy positiva n-dimensional e $U_{1}, \ldots, U_{k}$ são as integrais caudais de medidas de Lévy em $[0 ; \infty[$, então, a função $U$ definida acima é uma integral caudal de um processo de Lévy n-dimensional com saltos positivos possuindo marginais $U_{1}, \ldots, U_{n}$. 
A cópula de Lévy independente no caso multivariado possui a seguinte forma:

$$
C_{\perp}^{\mathcal{L}}\left(x_{1}, \cdots, x_{n}\right)=x_{1} 1_{\left\{x_{2}=\infty, \ldots, x_{n}=\infty\right\}}+\cdots+x_{n} 1_{\left\{x_{1}=\infty, \ldots, x_{n-1}=\infty\right\}},
$$

e a cópula de Lévy no caso de dependência completa é

$$
C_{\|}^{\mathcal{L}}\left(x_{1}, \cdots, x_{n}\right)=\min \left(x_{1}, \cdots, x_{n}\right) .
$$

Finalmente, é possível apresentar uma versão multidimensional da Proposição 4.6:

Proposição 4.7 Seja $\phi(u)$ uma função estritamente descrescente $\phi:[0 ; \infty] \longrightarrow$ $[0 ; \infty]$, tal que, $\phi(0)=\infty$ e $\phi^{-1}$ possui derivadas de ordem igual a $n$ em $] 0 ; \infty[$ com sinal que alterna, i.e., $(-1)^{k} \frac{d^{k} \phi^{-1}(t)}{d t^{k}}>0$. Assim,

$$
C^{\mathcal{L}}\left(x_{1}, \cdots, x_{n}\right)=\phi^{-1}\left(\phi\left(x_{1}\right)+\cdots+\phi\left(x_{n}\right)\right)
$$

define uma cópula de Lévy positiva n-dimensional.

\subsection{Cópulas de Processos de Lévy Generalizados}

É possível estender os conceitos, então, estabelecidos para os processos de Lévy que apresentam saltos tanto positivos como negativos, i.e., processos de Lévy generalizados, como segue:

Definição 4.12 $C^{\mathcal{L}}(x, y):[-\infty ; \infty]^{2} \longrightarrow[-\infty ; \infty]$ é uma cópula de Lévy se satisfazer as seguintes propriedades:

1. $C^{\mathcal{L}}$ é 2-crescente;

2. $C^{\mathcal{L}}(0, x)=C^{\mathcal{L}}(x, 0)=0, \forall x$;

3. $C^{\mathcal{L}}(x, \infty)-C^{\mathcal{L}}(x,-\infty)=C^{\mathcal{L}}(\infty, x)-C^{\mathcal{L}}(-\infty, x)=x$.

Por meio desta definição é possível observar que a cópula de Lévy positiva pode ser estendida para uma cópula de Lévy ao estabelecer que $C^{\mathcal{L}}(x, y)=0$ se $x<0$ ou $y<0$. É possível construir modelos com cópulas de Lévy quando a cópula de Lévy e as integrais caudais são relativamente suaves e possuem densidade. 


\subsubsection{Medidas de Lévy com Densidades}

Considere, inicialmente, a idéia de uma integral caudal de uma medida de Lévy definida em $\mathbb{R}$.

Definição 4.13 Seja $\nu$ uma medida de Lévy em $\mathbb{R}$. A integral caudal de $\nu$ é uma função $U: \overline{\mathbb{R}} \backslash\{0\} \longrightarrow[0 ; \infty]$ definida como

$$
\begin{aligned}
U(x) & =\nu([x ; \infty[), x \in] 0 ; \infty[, \\
U(x) & =-\nu([-\infty ; x[), x \in]-\infty ; 0[, \\
U(\infty) & =U(-\infty)=0 .
\end{aligned}
$$

Os sinais foram estabelecidos de tal maneira que a integral caudal é uma função decrescente tanto para $x \in] 0 ; \infty[$, como para $x \in]-\infty ; 0[$. Este resultado permite a construção de densidades de Lévy bidimensionais por meio de cópulas de Lévy suficientemente suaves e densidades de Lévy unidimensionais.

Proposição 4.8 Seja $C^{\mathcal{L}}$ uma cópula de Lévy bidimensional, contínua em $\overline{\mathbb{R}}^{2}$, tal que, $\frac{\partial C^{\mathcal{L}}(u, v)}{\partial u \partial v}$ existe em $\mathbb{R}^{2}$ e, seja $U_{1}$ e $U_{2}$ as integrais caudais unidimensionais com densidades $\nu_{1}$ e $\nu_{2}$. Assim,

$$
\left.\frac{\partial C^{\mathcal{L}}(u, v)}{\partial u \partial v}\right|_{u=U_{1}\left(x_{1}\right), v=U_{2}\left(x_{2}\right)} \nu_{1}\left(x_{1}\right) \nu_{2}\left(x_{2}\right)
$$

é a densidade de Lévy de uma medida de Lévy com densidades de Lévy marginais $\nu_{1} e$ $\nu_{2}$

\subsubsection{Cópulas de Lévy Generalizada}

Se a cópula de Lévy e as integrais caudais marginais não forem suficientemente suaves, a integral caudal multidimensional deve substituir a densidades de Lévy. Dado que a singulariedade encontra-se no centro do domínio de interesse (zero) cada canto da medida de Lévy deve ser trabalhada separadamente, i.e., no caso unidimensional é necessário duas integrais caudais: $U^{+}$e $U^{-}$e, no caso bidimensional, quatro integrais caudais: $U^{++}, U^{+-}, U^{-+}$e $U^{--}$. 
Definição 4.14 Seja $\nu$ uma medida de Lévy em $\mathbb{R}$. Esta medida apresenta duas integrais caudais, $U^{+}:[0 ; \infty] \longrightarrow[0 ; \infty]$ para a parte positiva $e, U^{-}:[-\infty ; 0] \longrightarrow$ $[-\infty ; 0]$ para a parte negativa, definidas como:

$$
\begin{aligned}
& U^{+}(x)=\nu([x ; \infty[), x \in] 0 ; \infty[, \\
& U^{+}(0)=\infty, U^{+}(\infty)=0, \\
& \left.\left.\left.U^{-}(x)=\nu(]-\infty, x\right]\right), x \in\right]-\infty ; 0[, \\
& U^{-}(0)=-\infty, U^{-}(-\infty)=0 .
\end{aligned}
$$

Seja $\nu$ uma medida de Lévy em $\mathbb{R}^{2}$ com integrais caudais marginais $U_{1}^{+}, U_{1}^{-}, U_{2}^{+}$e $U_{2}^{-}$. Esta medida possui quatro integrais caudais, i.e., $U^{++}, U^{+-}, U^{-+} e U^{--}$, sendo que, cada integral caudal é definida no seu respectivo quadrante, incluindo o eixo das coordenadas, como segue:

$$
\begin{aligned}
& U^{++}(x, y)=\nu([x ; \infty[\times[y ; \infty[), x, y \in] 0 ; \infty[ \\
& \left.U^{+-}(x, y)=\nu([x ; \infty[\times]-\infty, y]), x \in\right] 0 ; \infty[, y \in]-\infty ; 0[ \\
& \left.U^{-+}(x, y)=\nu(]-\infty, x\right] \times[y ; \infty[), x \in]-\infty ; 0[, y \in] 0 ; \infty[ \\
& \left.\left.\left.\left.\left.U^{--}(x, y)=\nu(]-\infty, x\right] \times\right]-\infty, y\right]\right), x, y \in\right]-\infty ; 0[.
\end{aligned}
$$

Se $x$ ou y for igual a $-\infty$ ou $+\infty$, a integral caudal correspondente é igual a zero e, se $x$ ou y for igual a 0 , a integral caudal satisfaz as seguintes condições marginais:

$$
\begin{aligned}
& U^{++}(x, 0)-U^{+-}(x, 0)=U_{1}^{+}(x) \\
& U^{-+}(x, 0)-U^{--}(x, 0)=U_{1}^{-}(x) \\
& U^{++}(0, y)-U^{-+}(0, y)=U_{2}^{+}(y) \\
& U^{+-}(0, y)-U^{--}(0, y)=U_{2}^{-}(y) .
\end{aligned}
$$

Quando uma medida de Lévy bidimensional atinge o eixo das coordenadas, sua integral caudal não é unicamente determinada, pois não fica claro qual das integrais caudais deve conter a densidade nos eixos. Desta maneira, conjuntos diferentes para as integrais caudais podem representar a mesma medida de Lévy. 
Teorema 4.7 Seja $\nu$ uma medida de Lévy em $\mathbb{R}^{2}$ com integrais caudais marginais $U_{1}^{+}(x), U_{1}^{-}(x), U_{2}^{+}(y)$ e $U_{2}^{-}(y)$. Assim, existe uma cópula de Lévy $C^{\mathcal{L}}$, tal que, as integrais caudais marginais são integrais de $\nu$, tal que,

$$
\begin{aligned}
& \left.U^{++}(x, y)=C^{\mathcal{L}}\left(U_{1}^{+}(x), U_{2}^{+}(y)\right), x, y \in\right] 0 ; \infty[ \\
& \left.U^{+-}(x, y)=C^{\mathcal{L}}\left(U_{1}^{+}(x), U_{2}^{-}(y)\right), x \in\right] 0 ; \infty[, y \in]-\infty ; 0[ \\
& \left.U^{-+}(x, y)=C^{\mathcal{L}}\left(U_{1}^{-}(x), U_{2}^{+}(y)\right), x \in\right]-\infty ; 0[, y \in] 0 ; \infty[ \\
& \left.U^{--}(x, y)=C^{\mathcal{L}}\left(U_{1}^{-}(x), U_{2}^{-}(y)\right), x, y \in\right]-\infty ; 0[.
\end{aligned}
$$

Se as integrais caudais marginais são absolutamente contínuas e, $\nu$ não atinge o eixo das coordenadas, a cópula de Lévy é unica. De maneira inversa, se $C^{\mathcal{L}}$ é uma cópula de Lévy e, $U_{1}^{+}(x), U_{1}^{-}(x), U_{2}^{+}(y)$ e $U_{2}^{-}(y)$ são integrais caudais unidimensionais de medidas de Lévy, então, as fórmulas acima definem oas integrais caudais da medida de Lévy bidimensional.

Prova A cópula de Lévy pode ser construída em cada um dos quatro quadrantes ao utilizar o Teorema 4.3 e o Lema 4.2. Com esta construção, é possível obter uma função 2 -crescente em cada um dos quadrantes, mas não para todo $\mathbb{R}^{2}$. Apesar disto, o fato de cada uma das quatro partes serem contínuas nos seus respectivos domínios, incluindo o eixo das coordenadas e igual à zero nos extremos das caudas garante que $C^{\mathcal{L}}$ é uma função 2-crescente em $\overline{\mathbb{R}}^{2}$.

Exemplo 4.6 - Processos de Lévy Independentes Dado que a medida de Lévy bidimensional de um processo de Lévy com componentes independentes é suportado pelo eixo das coordenadas, a correspondente cópula de Lévy não única. Neste caso, a Proposição 4.3 garante que para todo $x$ e $y$, tal que, $x y \neq 0$, integral caudal correspondente é igual a zero. Assim, $C^{\mathcal{L}}(x, y)=0$ se $x$ e $y$ forem finitos, as fórmulas (4.23) mostram que para cada cópula de Lévy que satisfaçam estas propriedades é uma cópula de Lévy independente. Exemplos de possíveis cópulas de Lévy independentes são $x 1_{\{y=\infty\}}+y 1_{\{x=\infty\}},-x 1_{\{y=-\infty\}}-y 1_{\{x=-\infty\}}$, etc.

\section{Exemplo 4.7 - Dependência Completa}

Ao estabelecer processos de Lévy com saltos de sinais arbitrários, é possível distinguir dois tipos de dependência completa. $\mathrm{O}$ 
primeiro caso (dependência completa positiva) existe um subconjunto crescente $S \subset$ $\mathbb{R}^{2}$, tal que, cada salto $\Delta X$ de um processo bidimensional pertence ao conjunto $S$. Nesta situação, ao utilizar o mesmo método da Proposição 4.4, tem-se

$$
\begin{aligned}
& U^{++}(x, y)=\min \left(U_{1}^{+}(x), U_{2}^{+}(y)\right) \\
& U^{--}(x, y)=\min \left(-U_{1}^{-}(x),-U_{2}^{-}(y)\right) \\
& U^{+-}(x, y)=U^{-+}(x, y) \triangleq 0 .
\end{aligned}
$$

Desta maneira, $C_{\uparrow \uparrow}^{\mathcal{L}}(x, y)=\min (|x|,|y|) 1_{\{x y \geq 0\}}$ é uma possível cópula para este processo. No caso de dependência completa negativa, os saltos de um processo bidimensional devem estar contidos em um subconjunto descrescente em $\mathbb{R}^{2}$, i.e., o conjunto $S$, tal que para todo dois vetores $\left\{v_{1}, v_{2}\right\} \in S$ e $\left\{u_{1}, u_{2}\right\} \in S$, tanto $\nu_{1}>u_{1}$ e $v_{2}<u_{2}$ ou, $\nu_{1}<u_{1}$ e $v_{2}>u_{2}$. Neste caso:

$$
\begin{aligned}
& U^{+-}(x, y)=-\min \left(U_{1}^{+}(x),-U_{2}^{-}(y)\right) \\
& U^{-+}(x, y)=-\min \left(-U_{1}^{-}(x), U_{2}^{+}(y)\right) \\
& U^{++}(x, y)=U^{--}(x, y) \triangleq 0
\end{aligned}
$$

e $C_{\uparrow \downarrow}^{\mathcal{L}}(x, y)=-\min (|x|,|y|) 1_{\{x y \leq 0\}}$.

Exemplo 4.8 Considere a cópula de Lévy apresentada na equação (4.21). Assim, é possível definir uma família de cópulas de Lévy com apenas um parâmetro, que inclui a independência e as dependências completas positiva e negativa.

$$
C_{\theta}^{\mathcal{L}}(u, v)=\left\{\begin{array}{cc}
\left(|u|^{-\theta}+|v|^{-\theta}\right)^{-1 / \theta} 1_{\{x y \geq 0\}} & \text { se } \theta>0 \\
-\left(|u|^{\theta}+|v|^{\theta}\right)^{1 / \theta} 1_{\{x y \leq 0\}} & \text { se } \theta<0
\end{array} .\right.
$$

Ao calcular os limites, tem-se que

$$
\begin{aligned}
& C_{\theta}^{\mathcal{L}} \longrightarrow C_{\uparrow \downarrow}^{\mathcal{L}}, \text { quando } \theta \longrightarrow-\infty \\
& C_{\theta}^{\mathcal{L}} \longrightarrow C_{\perp}^{\mathcal{L}}, \text { quando } \theta \longrightarrow 0 \\
& C_{\theta}^{\mathcal{L}} \longrightarrow C_{\uparrow \uparrow}^{\mathcal{L}}, \text { quando } \theta \longrightarrow \infty .
\end{aligned}
$$




\subsubsection{Construção de Cópulas de Lévy}

A construção de processos de Lévy generalizados pode ser bastante complexa, pois as Proposições 4.5 e 4.6 não podem ser estendidas para os casos generalizados. Uma possível solução é construir as cópulas de Lévy generalizadas por meio de agrupamento de cópulas de Lévy positivas, i.e., especificar a dependência dos saltos de diferentes sinais separadamente. Seja $C^{\mathcal{L}++}, C^{\mathcal{L}--}, C^{\mathcal{L}-+}$ e $C^{\mathcal{L}+-}$ cópulas de Lévy positivas e, considere a seguinte expressão:

$$
\begin{aligned}
C^{\mathcal{L}}(x, y)= & C^{\mathcal{L}++}\left(c_{1}|x|, c_{2}|y|\right) 1_{\{x \geq 0, y \geq 0\}}+C^{\mathcal{L}--}\left(c_{3}|x|, c_{4}|y|\right) 1_{\{x \leq 0, y \leq 0\}} \\
& -C^{\mathcal{L}+-}\left(c_{5}|x|, c_{6}|y|\right) 1_{\{x \geq 0, y \leq 0\}}-C^{\mathcal{L}-+}\left(c_{7}|x|, c_{8}|y|\right) 1_{\{x \leq 0, y \geq 0\}},
\end{aligned}
$$

sendo que $C^{\mathcal{L}}$ é 2 -crescente se as constantes $c_{1}, \ldots, c_{8}$ forem positivas. As condições marginais implicam em quatro restrições adicionais, e.g., para $x>0$ tem-se que $C^{\mathcal{L}}(x, \infty)-C^{\mathcal{L}}(x,-\infty)=c_{1} x+c_{5} x=x$, o que implica em $c_{1}+c_{5}=1$. As outras constantes $c_{3}+c_{7}=1, c_{2}+c_{8}=1 \mathrm{e} c_{4}+c_{6}=1$. Desta maneira, é possível reescrever a equação acima como:

$$
\begin{aligned}
C^{\mathcal{L}}(x, y)= & C^{\mathcal{L}++}\left(c_{1}|x|, c_{2}|y|\right) 1_{\{x \geq 0, y \geq 0\}}+C^{\mathcal{L}--}\left(c_{3}|x|, c_{4}|y|\right) 1_{\{x \leq 0, y \leq 0\}} \\
& -C^{\mathcal{L}+-}\left(\left(1-c_{1}\right)|x|,\left(1-c_{4}\right)|y|\right) 1_{\{x \geq 0, y \leq 0\}} \\
& -C^{\mathcal{L}-+}\left(\left(1-c_{3}\right)|x|,\left(1-c_{2}\right)|y|\right) 1_{\{x \leq 0, y \geq 0\}}
\end{aligned}
$$

define uma cópula de Lévy se as constantes $c_{1}, c_{2}, c_{3}, c_{4} \in[0 ; 1]$.

Para entender melhor o significado desta construção, considere as medidas de Lévy marginais $\nu_{1}$ e $\nu_{2}$ e observe a medida de Lévy bidimensional obtida pela cópula de Lévy [equação (4.25)]. A integral caudal do primeiro quadrante é

$$
U^{++}(x, y)=C^{\mathcal{L}++}\left(c_{1} U_{1}^{+}(x), c_{2} U_{2}^{+}(y)\right) .
$$

Isto significa que o primeiro quadrante de uma medida de Lévy corresponde a um processo de Lévy de saltos positivos, sendo que a cópula de Lévy será $C^{\mathcal{L}++}$ e as medidas de Lévy marginais são $c_{1} \nu_{1}(d x) 1_{\{x>0\}}$ e $c_{2} \nu_{2}(d y) 1_{\{y>0\}}$. Ao trabalhar os outros quadrantes de maneira análoga, é possível concluir que o processo de Lévy com a cópula apresentada pela equação (4.25) é a soma de quatro partes independentes, sendo que cada uma destas partes corresponde a um quadrante da medida de Lévy. Os componentes referentes à cópula de Lévy do primeiro quadrante apresentam apenas 
saltos positivos e possuem medidas de Lévy $c_{1} \nu_{1}(d x) 1_{\{x>0\}}$ e $c_{2} \nu_{2}(d x) 1_{\{x>0\}}$ sendo que a estrutura de dependência entre estas medidas é dada por $C^{\mathcal{L}++}$. A segunda parte independente refere-se ao segundo quadrante. O seu primeiro componente apresenta saltos positivos e possui medida de Lévy $\left(1-c_{1}\right) \nu_{1}(d x) 1_{\{x>0\}}$, enquanto que o segundo componente apresenta saltos negativos e possui medida de Lévy igual a $\left(1-c_{4}\right) \nu_{4}(d x) 1_{\{x<0\}}$. Este dois componentes são relacionados pela cópula de Lévy positiva $C^{\mathcal{L}+-}$. As outras duas partes independentes relativas ao terceiro e quarto quadrantes podem ser construídas de maneira análoga.

Dado que a proporção de saltos do primeiro componente que está relacionado aos saltos positivos do segundo componente não depende do tamanho dos saltos (depende apenas do seu sinal), a cópula apresentada pela equação (4.25) é denominada cópula de Lévy de proporções constantes. As cópulas de Lévy de processos estáveis sempre podem ser representadas pela forma dada pela equação (4.25). Por meio dos resultados apresentados no Exemplo 4.4, é possível mostrar que as cópulas de Lévy de processos estáveis são homogêneas de ordem 1 . Seja $C^{\mathcal{L}}(x, y)$ uma cópula de Lévy que satisfaça $C^{\mathcal{L}}(x, \infty)=x C^{\mathcal{L}}(1, \infty)$. Isto significa que

$$
\tilde{C}^{\mathcal{L}}(x, y)=C^{\mathcal{L}}\left(\frac{x}{C^{\mathcal{L}}(1, \infty)}, \frac{y}{C^{\mathcal{L}}(1, \infty)}\right), x, y \geq 0
$$

é uma cópula positiva de Lévy. Ao trabalhar os outros quadrantes de $C^{\mathcal{L}}$ da mesma maneira, é possível representar a cópula de Lévy de um processo estável pela forma apresentada na equação (4.25).

\subsubsection{Definições Estendidas aos Casos Multivariados}

Segue sumário das definições estendidas aos casos multivariados:

Definição 4.15 Cópula de Lévy Uma cópula de Lévy n-dimensional é uma função $C^{\mathcal{L}}: \overline{\mathbb{R}}^{n} \longrightarrow \overline{\mathbb{R}}$ com as seguintes propriedades:

1. $C^{\mathcal{L}}$ én-crescente;

2. $\quad C^{\mathcal{L}}$ é igual a zero se pelo menos um dos seus argumentos for igual a zero;

3. $C^{\mathcal{L}}$ possui marginais uniformes. 
Para construir uma medida de Lévy multidimensional por meio de cópulas de Lévy, é necessário introduzir o seguinte intervalo:

$$
\mathbf{I}(x)=\left\{\begin{array}{rl}
{[x ; \infty[,} & \text { se } x>0 \\
]-\infty ; x], & \text { se } x<0
\end{array} .\right.
$$

Ao utilizar esta notação, é possível construir as integrais caudais de uma medida de Lévy em qualquer ponto, salvo nos pontos que estão sob os eixos, como segue:

$$
\nu\left(\mathbf{I}\left(x_{1}\right) \times \cdots \times \mathbf{I}\left(x_{n}\right)\right)=(-1)^{\left(\operatorname{sgn} x_{1} \times \cdots \times \operatorname{sgn} x_{n}\right)} C^{\mathcal{L}}\left(U_{1}^{\operatorname{sgn} x_{1}}\left(x_{1}\right), \ldots, U_{n}^{\operatorname{sgn} x_{n}}\left(x_{n}\right)\right),
$$

sendo que $U_{1}^{+}, U_{1}^{-}, \ldots, U_{n}^{+}, U_{n}^{-}$são as integrais caudais marginais unidimensionais. $\mathrm{O}$ fato da não unicidade nos eixos pode ser resolvido como foi feito no caso bidimensional.

\subsection{Construindo Modelos Multivariados por Meio de Cópulas de Lévy}

Como foi observado anteriormente, a dependência multivariada de um processo de Lévy pode ser descrita por meio do conceito de cópula de Lévy, que exerce um papel semelhante ao das cópulas ordinárias para os processos de Lévy. Como no caso das cópulas ordinárias, a escolha apropriada da cópula de Lévy permite distinguir a dependência linear de outras formas de dependências para modelar os log-retornos de ativos financeiros. Esta distinção possui aplicações em modelos de gestão de risco, onde os movimentos conjuntos de saltos negativos nos log-retornos de ativos financeiros são importantes.

É possível modelar o preço de dois ativos financeiros $S_{t}^{1}$ e $S_{t}^{2}$ pelo exponencial de processos variância gama de Lévy. Um método seria representar $\ln S_{t}^{1}$ e $\ln S_{t}^{2}$ como dois componentes de um movimento browniano bivariado correlacionado, com tempo modificado por um único subordinador gama. Neste método, a estrutura de dependência é descrita por um único parâmetro $\rho$, que é coeficiente de correlação de Pearson de um MB. Assim, o processo que descreve estes ativos financeiros sempre apresentará o mesmo parâmetro de estrutura de saltos (i.e., $\kappa$ ) e, serão dependentes mesmo se os movimentos brownianos dos ativos financeiros forem decorrelacionados. 
Um método alternativo é construir o modelo por meio de cópulas de Lévy. Assim, tem-se que

$$
\begin{aligned}
X_{t} & =\ln S_{t}^{1}, \\
Y_{t} & =\ln S_{t}^{2},
\end{aligned}
$$

sendo que $X_{t}$ e $Y_{t}$ são dois processos gama arbitrários com dependência especificada pela cópula de Lévy $C^{\mathcal{L}}$. É possível assumir a forma da equação (4.25), i.e., cópula de Lévy de proporção constante. Neste caso, para especificar a estrutura da dependência completamente é necessário escolher 4 cópulas de Lévy positivas $C^{\mathcal{L}++}$ (dependência entre os saltos positivos de $X$ com os saltos positivos de $Y$ ), $C^{\mathcal{L}+-}$ (dependência entre os saltos positivos de $X$ com saltos negativos de $Y$ ), $C^{\mathcal{L}-+}$ (dependência entre os saltos negativos de $X$ com os saltos positivos de $Y$ ) e $C^{\mathcal{L}--}$ (dependência entre os saltos negativos de $X$ com os saltos negativos de $Y$ ). Adicionalmente, é preciso especificar quatro parâmetros positivos $c_{1}, c_{2}, c_{3}$ e $c_{4}$ que assumem valores entre 0 e 1 . Se todas as cópulas de Lévy positiva pertencerem a uma família de cópulas de Lévy parametrizadas com apenas um parâmetro, então, será necessário especificar um total de 8 parâmetros.

Exemplo 4.9 É possível assumir que os saltos positivos de $X_{t}$ são independentes dos saltos negativos de $Y_{t}$ e vice-versa. Entretanto, esta hipótese não é razoável quando os ativos financeiros são representadas por moedas. Adicionalmente, a estrutura de dependência de saltos positivos pode ser descrita pela família de Clayton de cópula de Lévy com parâmetro $\theta^{+}$e estrutura dependência dos saltos negativos pelo parâmetro $\theta^{-}$. Neste caso o modelo apresentara a seguinte forma

$$
\begin{aligned}
& \ln S_{t}^{1}=X_{t}=X_{t}^{+}-X_{t}^{-}, \\
& \ln S_{t}^{2}=Y_{t}=Y_{t}^{+}-Y_{t}^{-},
\end{aligned}
$$

sendo que $X_{t}^{+}$e $Y_{t}^{+}$representam os saltos positivos e $X_{t}^{-}$e $Y_{t}^{-}$os negativos. A cópula $\left\{X_{t}^{+}, Y_{t}^{+}\right\}_{t \geq 0}$ e $\left\{X_{t}^{-}, Y_{t}^{-}\right\}_{t \geq 0}$ são independentes, $\left\{X_{t}^{+}\right\}_{t \geq 0}$ e $\left\{Y_{t}^{+}\right\}_{t \geq 0}$ são dependentes com cópula de Lévy $C_{\theta^{+}}^{\mathcal{L}}$ e, $\left\{X_{t}^{-}\right\}_{t \geq 0}$ e $\left\{Y_{t}^{-}\right\}_{t \geq 0}$ são dependentes com cópula de Lévy $C_{\theta^{-}}^{\mathcal{L}}$. Assim, a estrutura de dependência de $\left\{X_{t}, Y_{t}\right\}_{t \geq 0}$ é descrita por dois parâmetros, $\theta^{+}$(descreve os saltos positivos) e $\theta^{-}$(descreve os saltos negativos). Este procedimento permite especificar estruturas de dependências diferentes para os saltos positivos e negativos, que seria impossível com um modelo baseado na subordinação 


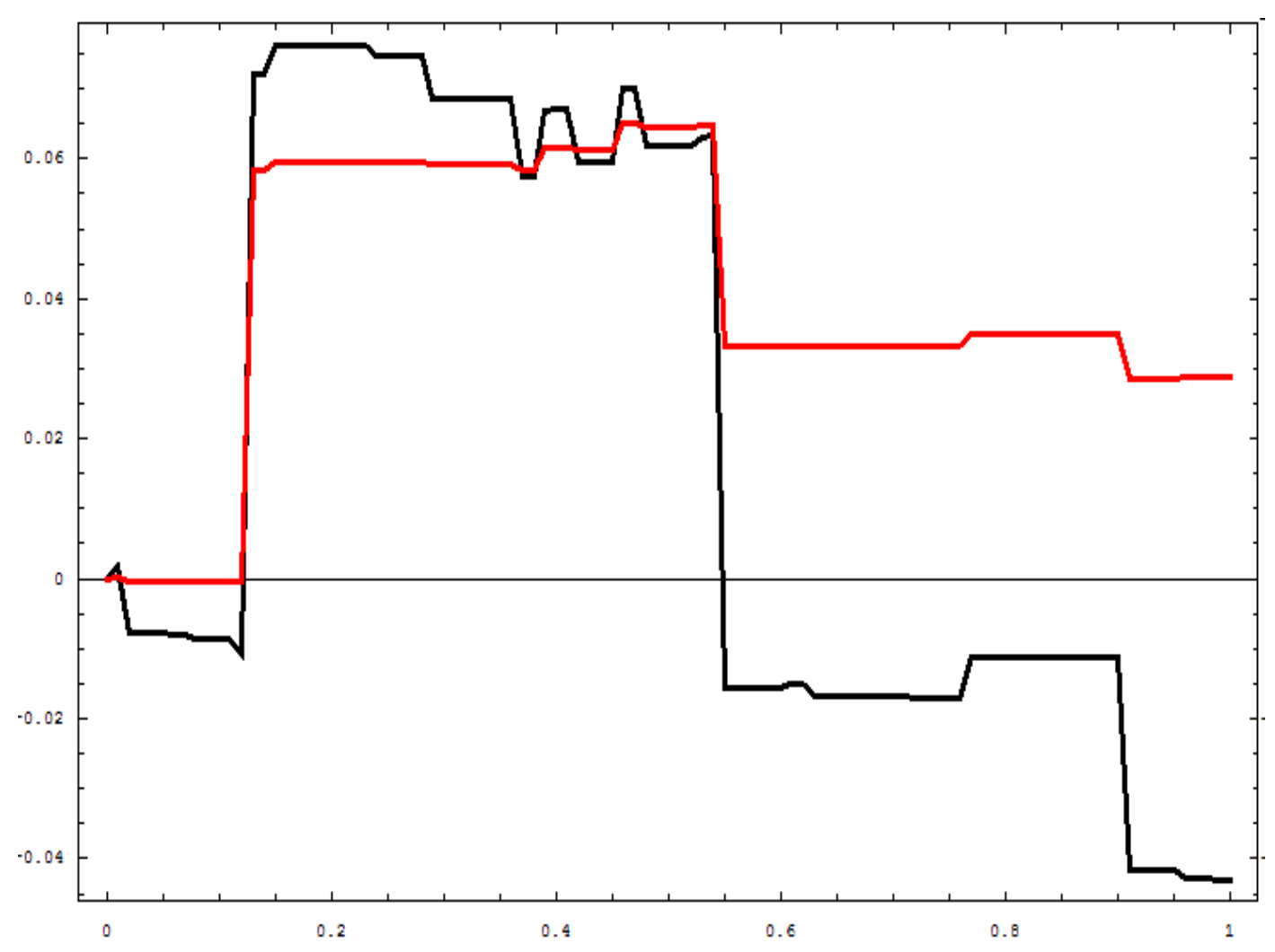

Figura 4.2. Trajetória de um processo variância gama bidimensional com estruturas de dependência diferentes para os movimentos positivos e negativos.

de um MB. Adicionalmente, neste modelo $\left\{X_{t}\right\}_{t \geq 0}$ e $\left\{Y_{t}\right\}_{t \geq 0}$ podem ser representados por processos variância gama completamente independentes (caso limite que ocorre quando $\theta^{+}$ou $\theta^{-}$aproximam-se de zero). Se $\left\{X_{t}\right\}_{t \geq 0}$ é um processo variância gama com parâmetros $\sigma, \theta$ e $\kappa$, então, $\left\{X_{t}^{+}\right\}_{t>0}$ será um subordinador gama com parâmetros $c_{+}=1 / \kappa \mathrm{e} \lambda_{+}=\left(\sqrt{\theta^{2}+2 \sigma^{2} / \kappa}-\theta\right) / \sigma^{2} \mathrm{e},\left\{X_{t}^{-}\right\}_{t \geq 0}$ será descrito pelos parâmetros $c_{-}=1 / \kappa$ e $\lambda_{-}=\left(\sqrt{\theta^{2}+2 \sigma^{2} / \kappa}+\theta\right) / \sigma^{2}$. A medida de Lévy do processo $\left\{X_{t}, Y_{t}\right\}_{t \geq 0}$ será sempre suportada pelo conjunto $\{\{x, y\}: x y \geq 0\}$, i.e., pelos saltos que estiverem localizados no primeiro e terceiro quadrantes. A Figura 4.2 descreve uma trajetória simulada ${ }^{19}$ de um processo variância gama, com parâmetros $\sigma=0,3$, $\theta=-0,1, \kappa=0,5, \theta^{+}=1$ e $\theta^{-}=5$. É possível comparar este modelo com o modelo obtido por subordinação browniana de um processo variância gama.

19 O algorítmo utilizado para simular o subordinador gama é o apresentado em Cont \& Tankov (2004b), p.201, e o valor de precisão da simulação foi $\tau=10^{2}$. 


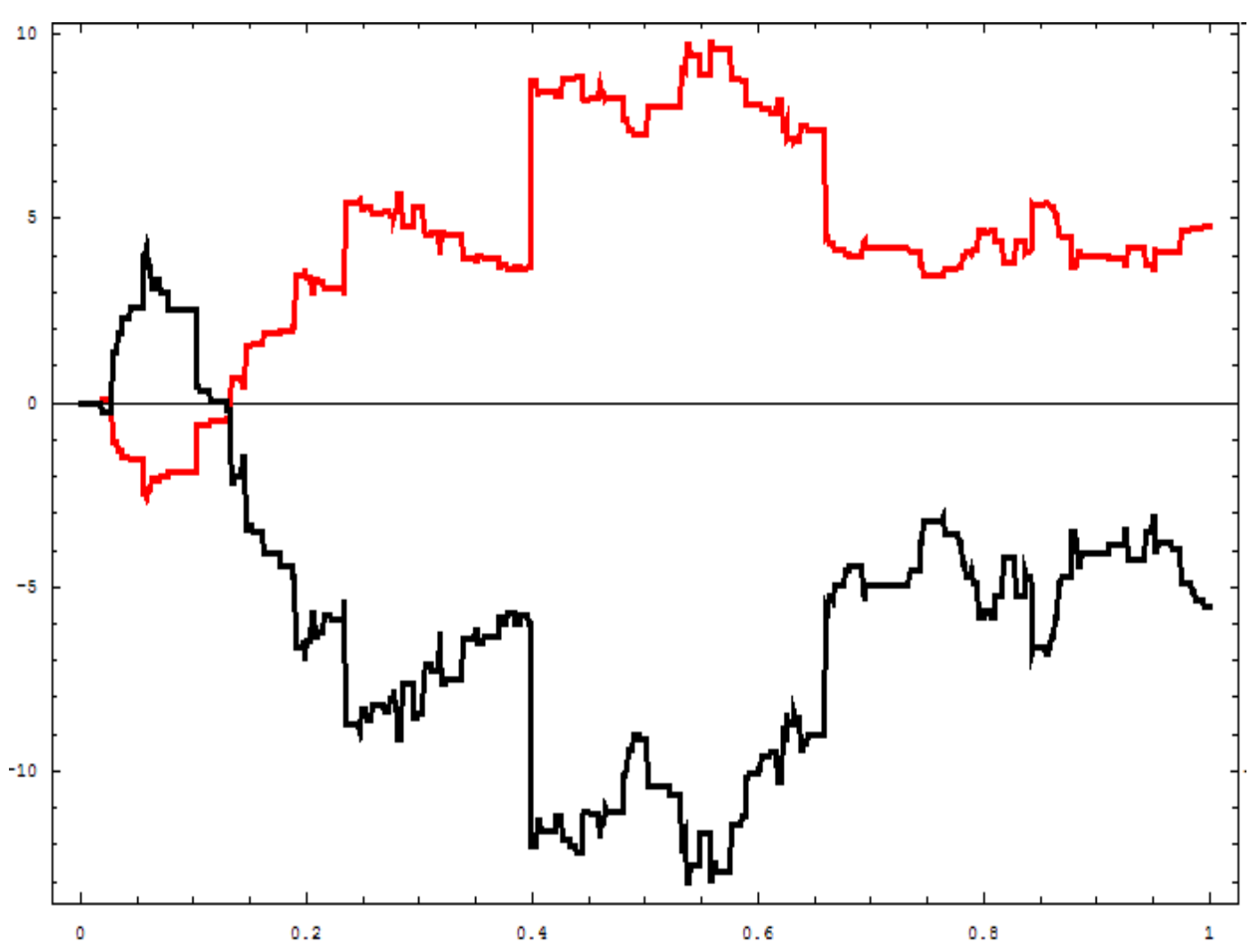

Figura 4.3. Trajetórias de dois processos variância gama simétricos sendo os saltos positivos copulados com os negativos e vice-versa.

Exemplo 4.10 No mercado de moedas (câmbio), os saltos negativos e positivos podem ser mais simétricos e apresentar uma maior dependência negativa. Assim, é possível representar a estrutura de dependência pela cópula de Lévy apresentada na equação (4.24). A Figura 4.3 representa a simulação de dois processos variância gama simétricos com $\lambda=1$ e $c=30$ e um parâmetro de dependência da família de Clayton igual $\theta=-3$.

Para $\theta>0$ os ativos financeiros apresentarão movimentos na mesma direção, enquanto que para $\theta<0$, os ativos financeiros apresentarão movimentos em direções diferentes. 


\section{Conclusões}

O presente estudo apresenta uma abordagem generalizadora de modelagem de séries temporais de ativos financeiros. Esta abordagem evita a discussão sobre se as séries temporais são efetivamente orientadas por um MB ou não [Osborne (1959), Pagan (1996), Campbell et al. (1997), Cont (2001) e Gourieroux (2001)], i.e., ao modelar as séries temporais por processos de Lévy é possível incluir características no modelo, tais como leptocurtose e assimetria na distribuição do processo, que geralmente não é possível por meio da modelagem via movimento browniano (MB). $\mathrm{O}$ Quadro 0.1 relaciona os principais conceitos de mais de três décadas de modelagem financeira e gerenciamento de risco e compara os modelos de difusão com os modelos de salto-difusão. Esta breve comparação mostra que, apesar de várias observações empíricas, computacionais e estatísticas que motivam a utilização dos modelos de difusão, os modelos com descontinuidades carregam informações qualitativas sobre os principais aspectos de hedge, replicação de carteiras e risco que são importantes na modelagem. Em certas circunstâncias os modelos de difusão apresentados nas equações $(0.3),(0.5)$ e (0.6) podem ser pertinentes, entretanto, estes modelos não apresentam as propriedades qualitativas desejadas e, portanto, carregam informações equivocadas sobre as flutuações dos preços e o risco resultante destas flutuações.

Uma das justificativas para a execução deste estudo, é que os modelos supra mencionados, podem ser entendidos como casos particulares dos processos de Lévy, que é o foco de todo o trabalho. Assim, os modelos de difusão são casos particulares dos modelos de salto-difusão. O Quadro 2.3 relaciona as principais categorias de processos abordados neste estudo, i.e., os processos gaussianos, os processos estáveis, os processos auto-similares e os processos de Lévy. Os modelos de salto-difusão, i.e., soma de um composto de Poisson e MB com t.d., e os modelos de difusão, i.e., MB com t.d. (drift), são casos particulares dos processos de Lévy. Os processos estáveis são processos com medidas de Lévy de atividade infinita e também são casos particulares dos processos de Lévy.

O estudo também apresentou os principais modelos de salto-difusão na literatura, i.e., os modelos de Kou e Merton, sendo que as principais características destes mo- 
delos podem ser observadas no Quadro 3.2. Estes modelos constituem a maneira mais fácil de se incluir descontinuidades no processo. Outra maneira bastante interessante é a construção de processos de Lévy por meio da subordinação browniana. A forma mais generalizadora desta abordagem é modelo hiperbólico generalizado $(\mathrm{GH})$. Espera-se que esta distribuição, que incorpora a maioria dos fatos empíricos observados nas séries financeiras [Prause (1999), Cont (2001) e Fajardo et al. (2001)], permita um ganho relativamente grande na qualidade dos indicadores de risco amplamente utilizados no gerenciamento de risco de operações de tesouraria pelo nosso mercado financeiro, o VaR (Value at Risk) [Jorion (1996)], bem como pela necessidade de se criarem modelos de precificação de instrumentos financeiros negociados em mercados pouco líquidos, i.e., baixo volume de negociação e pouca quantidade de preços de mercado disponíveis. Este modelo consiste na modificação do tempo de um MB por meio de um subordinador GIG. Boa parte das distribuições plausíveis para a modelagem de séries temporais financeiras, tais como a t de student, gaussiana, Laplace, hiperbólicas, NIG, VG são casos particulares do modelo GH. O Quadro 3.5 apresenta as principais características do modelo GH e o Quadro 3.6 ilustra que valores os parâmetros do modelo GH deve assumir para obter os principais casos particulares. O Exemplo 3.3 aborda o método de máxima verossimilhança para estimação dos parâmetros do modelo hiperbólico $(\lambda=1)$. Seria interessante em projetos futuros expandir o leque de métodos de estimação tais como os métodos GMM (Generalized Methods of Moments), ou o XMM (Extended Methods of Moments), proposto recentemente por Gourieroux et al. (2005), o que possibilitaria combinar a teoria sobre processos de Lévy apresentada neste estudo e técnicas inovadoras de estimação.

Muitos problemas em finanças quantitativas envolvem a especificação da distribuição conjunta de uma carteira composta por diversos ativos financeiros. Nos modelos gaussianos multivariados, a dependência entre os log-retornos é representada pelos coeficientes de correlação de Pearson. Quando são introduzidos saltos para representar estresses de mercado, são necessárias ferramentas específicas para especificar a dependência entre os saltos. Assim, o estudo apresentou alguns modelos [Madan \& Seneta (1990), Geman et al. (2001) e Carr \& Wu (2003)] que representam os ativos financeiros por movimentos brownianos subordinados ou por modelos de fatores orientados por processos compostos de Poisson, i.e., generalizaçõs dos mode- 
los de Merton e Kou. A modelagem por meio de subordinação browniano permite construir processos de Lévy analiticamente versáteis. Entretanto, a estrutura de dependência é um pouco inflexível, pois não permite a possibilidade de independência entre os log-retornos dos ativos financeiros e estabelecer dinâmicas diferentes para os ativos financeiros individuais. A modelagem por meio de inovações de Poisson é possível para modelos de dimensão pequena e quando os saltos ocorrerem simultaneamente no processo dos log-retornos de todos os ativos. A inclusão de inovações de Poisson independentes para cada um dos ativos financeiros pode superparametrizar o modelo, o que dificulta a estimação e exige uma quantidade de dados relativamente grande.

Fatos empíricos em finanças [Carr et al. (2002)] indicam que os saltos representam o risco sistêmico de mercado e não podem ser independentes entre os ativos que compõem a carteira em análise. Assim, uma maneira sistematizada de descrever a estrutura de dependência entre duas variáveis estocásticas é por meio de funções de cópulas. O estudo apresentou o desenvolvimento recente da teoria de cópulas [Kallsen \& Tankov (2005)], que estende o conceito da cópula ordinária, i.e., cópula de uma v.e., para o caso de processos de Lévy, i.e., a cópula de Lévy. A cópula de Lévy permite a construção de processos de Lévy multidimensionais. A construção de modelos por meio de cópulas de Lévy é uma abordagem que permite especificar a estrutura de dependência entre os log-retornos de maneira bastante flexível e, adicionalmente, permite especificar as distribuições marginais de cada um dos ativos financeiros individualmente. Técnicas de simulação de Monte Carlo possibilitaram realizar os exemplos apresentados no Capítulo 4 - Modelos Multidimensionais [Cont \& Tankov (2004b), Cap.6].

Aplicações dos processos de Lévy na área de finanças quantitativas são bastante extensas, e por si só já merecem um estudo individual. Assim, uma extensão natural do estudo seria a precificação de derivativos via transformadas de Esscher [Gerber \& Shiu (1994a)], pelo qual é possível formalizar consistentemente uma maneira de obter medidas neutralizadoras de risco, i.e., processos de Lévy que sejam martingales. Os conceitos apresentados neste estudo também seriam úteis para a precificação de outras opções, como as opções de taxas de juros, que utilizam processos estacionários com reversão à média, tal como o processo de Ornstein-Ühlenbeck 
[Prause (1999)], ou como as opções americanas que dependem da trajetória do preço até o vencimento da opção [Gerber \& Shiu (1994b)]. Outras extensões seriam os modelos gerais de precificação, tais como o APT e CAPM, que poderiam considerar hipóteses robustas, contemplando as reais características dos ativos financeiros. Outra extensão do estudo é abordar o gerenciamento de risco e a formação de estratégias de hedge. Os conceitos de utilidade, equivalente monetário determinístico e divergência direcionada de Kullback-Leibler (entropia relativa) combinados com as técnicas de controle ótimo são essenciais para estabelecer estratégias de mitigação de risco no contexto de mercados incompletos.

Os processos de Lévy, por considerar o intervalo de tempo estocástico entre um salto e outro, i.e., tempo estocástico, permitem modelar a inadimplência, que é essencial para o estudo do risco de crédito e dos derivativos de crédito, que são instrumentos utilizados em mercados incompletos para distribuir o risco de crédito entre as instituições do mercado financeiro no Brasil. Este risco trata principalmente do risco de não-recebimento dos fluxos de caixas terminais de um determinado ativo financeiro. $\mathrm{O}$ estudo do risco operacional que é o risco de incorrer em prejuízos em decorrências de falhas humanas ou computacionais nos processos operacionais está muito relacionado a eventos extremos [Embrechts et al. (1997)] e apresenta várias aplicações para a teoria de cópulas, no que diz respeito à modelagem da dependência entre os agentes de riscos.

Assim, de uma maneira geral, o Acordo da Basiléia II $^{20}$ vem orientando as instituições financeiras a realizarem análises quantitativas de riscos de mercado, crédito, liquidez e operacional [Cherubini et al. (2004)], exigindo que os modelos de risco sejam robustos às hipóteses tradicionais de mercado, principalmente em países emergentes como o Brasil, onde a economia não é considerada estável, o que significa que os modelos gaussianos nem sempre são adequados. Desta maneira, o trabalho não esgota o assunto, pois limita-se à apresentação dos conceitos relacionados aos processos de Lévy multidimensionais, à estimação dos parâmetros do p.g.d. e a análise da dependência entre os componentes de processos multidimensionais por meio de cópulas de Lévy.

Seguem as principais contribuições do autor:

1. Esclarecimento da Definição 1.11. [p.24]

20 Disponível em <http://www.bis.org/publ/index.htm $>$. 
2. Esclarecimento da Definição 1.12. [p.25]

3. Esclarecimento da Prova da Proposição 1.5. [p.29]

4. Esclarecimento da Prova da Propriedade 8 da Proposição 1.6. [p.31]

5. Esclarecimento da Prova da Proposição 2.4. [p.58]

6. Esclarecimento da Prova da Propriedade 2 da Proposição 2.5. [p.60]

7. Esclarecimento da Prova da Propriedade 3 da Proposição 2.5. [p.60]

8. Esclarecimento da Prova do Lema 2.1. [p.62]

9. Esclarecimento da Prova da Proposição 2.6. [p.63]

10. Esclarecimento da Prova da Proposição 2.7. [p.65]

11. Esclarecimento da Prova do Teorema 3.1. [p.93]

12. Esclarecimento da Prova do Teorema 3.2. [p.96]

13. Desenvolvimento da equação (3.18). [p.105]

14. Desenvolvimento da equação (3.19). [p.106]

15. Desenvolvimento da equação (3.20). [p.106]

16. Desenvolvimento da equação (3.21). [p.107]

17. Desenvolvimento da Proposição 3.2. [p.112]

18. Desenvolvimento da Prova da Proposição 3.2. [p.112]

19. Desenvolvimento da Proposição 3.3. [p.113]

20. Desenvolvimento da Prova da Proposição 3.3. [p.113]

21. Desenvolvimento da Proposição 3.4. [p.113]

22. Desenvolvimento da Prova da Proposição 3.4. [p.113]

23. Desenvolvimento da equação (3.33). [p.114] 
24. Desenvolvimento da equação (3.34). [p.115]

25. Desenvolvimento da equação (3.35). [p.115]

26. Esclarecimento da equação (3.36). [p.115]

27. Esclarecimento da equação (3.37). [p.115]

28. Esclarecimento da equação (3.38). [p.115]

29. Desenvolvimento da equação (3.39). [p.116]

30. Desenvolvimento da equação (4.3). [p.131]

31. Desenvolvimento da equação (4.4). [p.134]

32. Desenvolvimento do algoritmo referente ao Quadro 3.8. [p.180]

33. Desenvolvimento do algoritmo referente ao Quadro 4.1. [p.182]

34. Desenvolvimento do algoritmo referente à Figura 0.1. [p.183]

35. Desenvolvimento do algoritmo referente à Figura 0.2. [p.184]

36. Desenvolvimento do algoritmo referente à Figura 1.1. [p.184]

37. Desenvolvimento do algoritmo referente à Figura 1.2. [p.185]

38. Desenvolvimento do algoritmo referente à Figura 2.1. [p.187]

39. Desenvolvimento do algoritmo referente à Figura 2.2. [p.188]

40. Desenvolvimento do algoritmo referente à Figura 4.1. [p.190]

41. Desenvolvimento do algoritmo referente à Figura 4.2. [p.191]

42. Desenvolvimento do algoritmo referente à Figura 4.3. [p.192] 


\section{Referências Bibliográficas}

ABRAMOWITZ, M.; STEGUN, I. Handbook of Mathematical Functions. New York: Dover, 1968.

AGGOUN, Lakhdar; ELLIOT, Robert. Measure Theory and Filtering: introduction and applications. Cambridge: Cambridge, 2004.

APARÍCIO, F.M.; ESTRADA, J. Empirical Distributions of Stock Returns: European Securities Markets. European Journal of Finance. [S.1.], 7:1-21, 2001.

ARDITTI, Fred D. Derivatives: a comprehensive resource for options, futures, interest rate swaps, and mortgage securities. Boston: Harvard Business, 1996.

ARFKEN, George B.; WEBER, Hans J. Mathematical Methods for Physicists. 6.ed. Oxford: Elsevier, 2005.

AVELlanedA, Marco; LAURENCE, Peter. Quantitative Modeling of Derivative Securities: from theory to practice. New York: Chapman \& Hall/CRC, 2000.

BACHELIER, L. Théorie de la Spéculation. Annales de l 'Ecole Normale Supérieure. Paris, 3:21-86, 1900.

BARNDORFF-NIELSEN, O.E. Exponentially Decreasing Distributions for the Logarithm of Particle Size. Procceding of the Royal Society of London. London, A353:401-419, 1977.

. Normal Inverse Gaussian Distributions and Stochastic Volatility Modelling. Scandinavian Journal of Statistics. [S.1.], 24:1-13, 1997. $68,1998$.

. Processes of Normal Inverse Gaussian Type. Finance Stochastics. [S.1.], 2:41-

BATES, David S. The Crash of '87: Was It Expected? The Evidence from Options Markets. The Journal of Finance. [S.1.], 46(3):1009-1044, 1991.

. Jumps and Stochastic Volatility: Exchange Rate Processes Implicit in Deutsche Market Options. Review of Financial Studies.[S.1.], 9(1):69-107, 1996.

. Post ' 87 Crash Fears in the S\&P 500 Futures Option Market. Journal of Econometrics. [S.1.], 94:181-239, 2000.

BAXTER, Martin W.; RENNIE, Andrew, J. O. Financial Calculus: An introduction to derivative pricing. Cambridge: Cambridge, 2003. 
BLACK, Fischer; SCHOLES, Myron. The Pricing of Options and Corporate Liabilities The Journal of Political Economy. Chicago, 81(3):637-654, 1973.

CAMPBELL, John Y. et al. The Econometrics of Financial Markets. Princeton: Princeton, 1997.

CARR, Peter et al. The Fine Structure of Asset Returns: An Empirical Investigation. Journal of Business. [S.1.], 75(2):305-332, 2002.

CARR, Peter et al. Stochastic Volatility for Lévy Processes. Mathematical Finance. [S.1.], 13(3):345-382, 2003.

CARR, Peter; WU, Liuren. What Type of Process Underlies Options? A Simple Robust Test. The Journal of Finance. [S.1.], 58(6):2581-2610, 2003.

CHAN, Terence. Pricing Contingent Claims on Stocks Driven by Lévy Processes. The Annals of Applied Probability. [S.1.], 9(2):504-528, 1999.

CHERUBINI, Umberto et al. Copulas Methods in Finance. London: Wiley, 2004.

COCHRANE, John H. Asset Pricing. 2.ed. Princeton: Princeton, 2005.

CONT, Rama. Empiral Properties of Asset Returns: Stylized Facts and Statistical Issues. Quantitative Finance. [S.1.], 1:223-236, 2001.

CONT, Rama; TANKOV, Peter. Nonparametric Calibration of Jump-Diffusion Option Pricing Models. Journal of Computational Finance. [S.1.], 7:1-49, 2004.

2004 .

CONT, Rama et al. Option Pricing Models with Jumps: Integro-Differential Equations and Inverse Problems. Artigo em apresentação, 2004.

COX, John C.; ROSS, Stephen A. The Valuation of Options for Alternative Stochastic Processes. Journal of Financial Economics. North-Holland, 3:145-166, 1976.

COX, John et al. Option Pricing: A Simplified Approach. Journal of Financial Economics. North-Holland, 7:229-263, 1979.

DAVIDSON, Russell; MACKINNON, James G. Econometric Theory and Methods. Oxford: Oxford, 2003.

DERMAN, E; KANI, I. Riding on a Smile. RISK. [S.1.], 7:32-39, 1994. 
DIXIT, Avanash K. The art of smooth pasting, v. 55, Fundamentals of pure and applied economics. Amsterdam: Harwood Academic, 1993.

DUPIRE, B. Pricing with a Smile, RISK. [S.1.], 7:18-20, 1994.

DUARTE, J.; MENDEZ, B.V.M. Robust Estimation for ARCH Models. Brazilian Review of Econometrics. [S.1.], 19:139-180, 1999.

EBERLEIN, Ernst. Application of Generalized Hyperbolic Lévy Motions in Finance. In: BARNDORFF-NIELSEN, Ole E. et al. (org.). Lévy Processes: theory and applications. Boston: Birkhäuser, 319-336, 2001.

EBERLEIN, Ernst et al. New Insights into Smiles, Mispricing and Value at Risk: The Hyperbolic Model. Journal of Business. [S.1.], 71(3):371-405, 1998.

EBERLEIN, Ernst; KELLER, Ulrich. Hyperbolic Distributions in Finance. Bernoulli. [S.1.], 1:281-299, 1995.

EBERLEIN, Ernst; PRAUSE, Karsten. The Generalizes Hyperbolc Model: Financial Derivatives and Risk Measures. In: GEMAN, H. et al. (org.). Mathematical Finance - Bachelier Congress 2000. [S.1.]: Springer-Verlag, 245-267, 2000.

EBERLEIN, Ernst; RAIBLE, Sebastian. Some Analytic Facts on the Generalized Hyperbolic Model. In: CASACUBERTA, C. et al. (org.). European Congress of Mathematics (Progress in Mathematics, v.202). [S.1.], 2:189-202, 2001.

EINSTEIN, Albert. Investigations on the Theory of the Brownian Movement. [S.1.]: Courier Dover, 1905.

EMBRECHTS, Paul et al. Modelling Extremal Events, vol. 33 of Applications of Mathematics. Berlin: Springer, 1997.

EMBRECHTS, Paul; MAEJIMA, Makoto. Selfsimilar Processes, Princeton Series in Applied Mathematics. Princeton: Princeton, 2002.

ENDERS, Walter. Applied Econometric Time Series. 2.ed. New York: Wiley, 2003.

FAJARDO, José et al. Lévy Process and the Brazilian Market. Brazilian Review of Econometrics. [S.1.], 21(2):263-289, 2001.

FAJARDO, José; FARIA, Aquiles. Generalized Hyperbolic Distributions and Brazilian Data. Brazilian Review of Econometrics. [S.1.], 24(1):1-21, 2004.

FELLER, W. An Introduction to Probability Theory and its Applications, vol II. New York: Wiley, 1971. 
FERNANDEZ, Pedro Jesus. Medida e Integração. Rio de Janeiro: Projeto Euclides, 2002.

FOUQUE, Jean-Pierre et al. Derivatives in Financial Markets with Stochastic Volatility. Cambridge: Cambridge, 2001.

GEMAN, Hélyette et al. Time Changes for Lévy Processes. Mathematical Finance. [S.1.], 11(1):79-96, 2001.

GERBER, Hans U.; SHIU, Elias, S.W. Option Pricing by Esscher Transforms. Transactions of Society of Actuaries. [S.1.], 46:99-191, 1994b.

. Martingale Approach to Pricing Perpetual American Options. ASTIN Bulletin. [S.1.], 24(2): 195-220, 1994.

GOURIEROUX, Christian; JASIAK, Joann. Financial Econometrics. Princeton: Princeton, 2001.

GOURIEROUX, Christian et al. Efficient Derivative Pricing by Extended Method of Moments. Discussion Paper. University of St. Gallen, 2005.

GREENE, Willian H. Econometric Analysis. 5.ed. Upper Saddle River: Prentice-Hall, 2002.

HAMILTON, James D. Time Series Analysis. Princeton: Princeton, 1994.

HARRISON, J. Michael; KREPS, David M. Martingales and Arbitrage in Multiperiod Securities Markets. Journal of Economic Theory. [S.1.], 20:381-408, 1979.

HARRISON, J. Michael; PLISKA, Stanley R. A Stochastic Calculus Model of Continuous Trading: Complete Markets. Stochastic Processes and Their Applications. North-Holland, 15:313-316, 1983.

. Martingales and Stochastic Integrals in the Theory of Continuous Trading. Stochastic Processes and Their Applications. North-Holland, 11:215-260, 1981.

HALGREEN, Christian. Self-Decomposability of the Generalized Inverse Gaussian and Hyperbolic Distributions. Probability Theory and Related Fields. [S.1.], 47(1):13$17,1979$.

HESTON, S. A Closed-Form Solution for Options with Stochastic Volatility with Applications to Bond and Currency Options. Review of Financial Studies. [S.1.], 6:327-343, 1993.

HULL, John; WHITE, Alan. The Pricing of Options on Assets with Stochastic Volatilities. Journal of Finance. [S.1.], 42(2):281-300, 1987. 
JÄQUEL, Peter. Monte Carlo Methods in Finance. Indianopolis: Wiley, 2002.

JACOD, Jean; SHIRYAEV, Albert N. Limit Theorems for Stochastic Processes. 2.ed. Berlim: Springer-Verlag, 2002.

JOE, Harry. Relative entropy measures of multivariate dependence. Journal of the American Statistical Association. [S.1.], 84:157-164, 1989.

. Multivariate Models and Dependence Concepts, Monographs on Statistics and Applied Probability, v.73. London: Chapman \& Hall/CRC, 1997.

JORION; Philippe. Value at Risk: The New Benchmark for Controlling Derivatives Risk. [S.1.]: Irwin Professional, 1996.

KARATZAS, Ioannis; SHREVE, Steven. Brownian Motion and Stochastic Calculus. 2.ed. New York: Springer-Verlag, 1997.

KALLENBERG, Olav. Foundations of Modern Probability. 2.ed. New York: Springer, 2002.

KALLSEN, Jan; TANKOV, Peter. Characterization of Dependence of Multidimensional Lévy Processes using Lévy copulas. Artigo em apresentação, 2005.

KOLEV, Nikolai et al. Copula Associated to Order Statistics. Relatório do Departamento de Estatística IME-USP. São Paulo: RT-MAE 2004-16, 2004.

Copulas: a Review and Recent Developments. Relatório do Departamento de Estatística IME-USP. São Paulo: RT-MAE 2005-7, 2005.

KOLEV, Nikolai; ANJOS, Ulisses. Copulas with Given Nonoverlapping Multivariate Marginals. Relatório do Departamento de Estatística IME-USP. São Paulo: RTMAE 2005-2, 2005.

. Representation of Bivariate Copulas via Local Measure of Dependence. Relatório do Departamento de Estatística IME-USP. São Paulo: RT-MAE 2005-3, 2005.

KONOPEN, Ismo. Analytic Approach to the Problem of Convergence of Truncated Lévy Flights Toward Gaussian Stochastic Process. Physical Review E. [S.1.], 52(1): 1197-1199, 1995.

KOU, Steven G. A Jump-Diffusion Model for Option Pricing. Management Science. [S.1.], 48(8):1086-1101, 2002.

KOU, Steven G.; WANG, Hui. Option Pricing Under a Double Exponential Jump Difusion Model. Management Science. [S.1.]: 50(9):1178-1192, 2004. 
LEWIS, Alan L. Option Valuation under Stochastic Volatility with Mathematica Code. Newport Beach: Finance Press, 2000.

LINDSKOG, Filip; McNEIL, Alexander J. Common Poisson Shock Models Applications to Insurance and Credit Risk Modelling. Artigo em apresentação, 2001. Disponível em $<$ www.risklab.ch $>$.

LUENBERGER, David G. Investment Science. Oxford: Oxford, 1997.

MADAN, Dilip B. et al.The Variance Gamma Processes and Option Pricing. European Finance Review. [S.1.], 2:79-105, 1998.

MADAN, Dilip B.; SENETA, E. Chebyshev Polynomial Approximations and Characteristic Function Estimation. Journal of the Royal Statistical Society. London, Series B, 49(2):163-169, 1987.

. The Variance Gamma (V.G.) Model for Share Market Returns. Journal of Business. [S.1.], 63:511-524, 1990.

MANDELBROT, B. The Variation of Certain Speculative Prices. The Journal of Business. [S.1.], 36:394-419, 1963.

MARDIA, K.V. et al. Multivariate Analysis, Probability and Mathematical Statistics. [SI]: Academic Press, 1980.

MERTON, Robert, C. Theory of Rational Option Pricing. The Bell Journal of Economics and Management Science. [S.1.], 4(1):141-183, 1973.

MERTON, Robert, C. Option Pricing when the Underlying Stock Returns are Discontinuous. Journal of Financial Economics. [S.1.], 3:125-144, 1976.

MILLS, Terence C. The Econometric Modelling of Financial Time Series. 2.ed. Cambridge: Cambridge, 1999.

MUSIELA, Marek; RUTKOWSKI, Marek. Martingales Methods in Financial Modelling: Theory and Applications. New York: Springer. 1997.

NEFTCI, Salih N. An Introduction to the Mathematics of Financial Derivatives. 2.ed. San Diego: Academic, 2000.

NELSEN, Roger B. An Introduction to Copulas. New York: Springer, 1999.

NELSON, Daniel, B. ARCH Models as Difusion Approximations. Jounal of Econometrics. North-Holland, 45:7-38, 1990. 
$\emptyset$ KSENDAL, Bernt. Stochastic Differential Equations: an introduction with application. 6.ed. New York: Springer, 2003.

$\emptyset$ KSENDAL, Bernt; LUND, Diderik. Stochastic Models and Options Values. New York: Elsevier Science Publishers, 1991.

ØKSENDAL, Bernt; SULEM, Agnès. Applied Stochastic Control of Jump Diffusions. New York: Springer, 2004.

OREY, Steven. On Continuity Properties of Infinitily Divisible Distributions. Annals of Mathematical Statistics. [S.1.], 39(3):936-937, 1968.

OSBORNE, M.F.M. Brownian motion in the Stock Market. Operations Research. [S.1.], 7:145-173, 1959.

PAGAN, Adrian. The Econometrics of Financial Markets. Journal of Empirical Finance. [S.1.], 3:15-102, 1996.

PRAETZ, Peter D. The Distribution os Share Price Changes. The Journal of Business. Chicago, 45(1):49-55, 1972.

PRATT, John W. et al. Introduction to Statistical Decision Theory. Cambridge: MIT, 1996.

PRAUSE, Karsten. The Generalized Hyperbolic Model: Estimation, Financial Derivatives, and Risk Measures. Freiburg, 1999. Tese de Doutorado - Faculdade de Matemática da Universidade de Freiburg, Alemanha.

PRESS, S.J. A Compound Events Model for Security Prices. Journal of Business. [S.1.], 40:317-335, 1967.

PRESS, William H. et al. Numerical Recipes in C++: The Art of Scientific Computing. 2.ed. Cambridge: Cambridge, 2002.

PROTTER, Philip. Stochastic Integration and Differential Equations: a new approach. Berlin: Springer-Verlag, 1990.

. A Partial Introduction to Financial Asset Pricing Theory. Stochastic Processes and Their Applications. [S.1.], 91:169-203, 2001.

RAIBLE, Sebastian. Lévy Processes in Finance: Theory, Numerics, and Empirical Facts. Freiburg, 2000. Tese de Doutorado - Faculdade de Matemática da Universidade de Freiburg, Alemanha.

REBONATO, R. Volatility and Correlation in Pricing of Equity, FX and Interest Rate Options. Indianopolis: Wiley, 1999. 
REVUZ, D.; YOR, M. Continuous Martingale and Brownian Motion. Berlin: Spring, 1999.

ROSS, Sheldon M. Applied Probability Models with Optimization Applications. New York: Dover, 1970.

. Stochastic Processes. 2.ed. New York: Wiley, 1996.

. A First Course in Probability. 6.ed. Upper Saddle River: Prentice-Hall, 2002.

ROSS, Stephen A. Options and Efficiency. Quaterly Journal of Economics. [S.1.], 90(1):75-99, 1976.

SAMORODNITSKY, G.; TAQQU, M. Stable Non-Gaussian Random Processes. New York: Chapman \& Hall/CRC, 1994.

SAMUELSON, Paul. Rational Theory of Warrant Pricing. Industrial Management Review. [S.1.], 6:13-32, 1965.

SATO, Ken-Iti. Lévy Processes and Infinitely Divisible Distributions. Cambridge: Cambridge, 1999.

SHIRYAEV, Albert N.; PROKHOROV, Yu. V. Probability Theory III. Stochastic Calculus. Berlim: Springer, 1989.

SIQUEIRA, José O. Determinação Entrópica do Preço Racional da Opção Européia Simples Ordinária sobre Ação e Bond: uma aplicação da teoria da informação em finanças em condição de incerteza. São Paulo, 1999. Tese de Doutorado - Faculdade de Economia, Administração e Contabilidade da Universidade de São Paulo.

S $\emptyset$ RENSEN, M. et al. Diffusion-Type Models with Given Marginal Distribution and Autocorrelation Function. Bernoulli. [S.1.], 2:191-220, 2005.

SPANOS, Aris. Probability Theory and Statistical Inference: econometric modeling with observational data. Cambridge: Cambridge, 2000.

TSAY, Ruey S. Analysis of Financial Time Series. 2.ed. Indianopolis: Wiley, 2005.

UEBERHUBER, C.W. Numerical Computation 2: Methods Sofware and Analysis. Berlin: Springer-Verlag, 1997.

WOLFRAM, Stephen. Mathematica Book. 5.ed. Champaign: Wolfram Media, 2003. 


\title{
Apêndice A \\ Algoritmos em Mathematica 5.1
}

\author{
A.1 Algoritmo referente ao Quadro 3.8 \\ $<<$ Graphics 'Graphics' \\ $<<$ Statistics 'Descriptivestatistics' \\ $<<$ Statistics 'ContinuousDistributions' \\ SetDirectory ["C: \data \dis"]; \\ data=Import ["base.csv"] ; \\ $\operatorname{Do}[\operatorname{asset}[j]=\operatorname{data}[[A l l, j]],\{j, \operatorname{Dimensions}[\operatorname{data}][[2]]\}]$; \\ $\mathrm{K}\left[\right.$ Lambda_, $\left.\mathrm{x}_{-}\right]:=0.5 \mathrm{NIntegrate}\left[\mathrm{y}^{\wedge}(\right.$ Lambda-1) \\ $\operatorname{Exp}\left[-0.5 \times\left(y+y^{\wedge}(-1)\right)\right],\{y, 0, \operatorname{Infinity}\}$, \\ Method->Gausskronrod, AccuracyGoal->6, \\ MaxRecursion->12] \\ hyp [x_,Alpha_,Beta_,Delta_,Mu_]:=Sqrt [Alpha^2-Beta^2] \\ / (2 Delta Alpha K[1, Delta \\ Sqrt [Alpha^2 -Beta^2]]) Exp [-Alpha \\ Sqrt [Delta^2+(x-Mu)^2]+Beta $(x-M u)]$ \\ L $\left[y_{-}\right.$, Alpha_,Beta_,Delta_,Mu_] :=Sum[Log [ \\ Sqrt[Alpha^2-Beta^2]]-Log[2 Delta Alpha \\ K[1, Delta Sqrt[Alpha^2 -Beta^2]]]-Alpha \\ Sqrt [Delta^2+(y[ [i] ] -Mu)^2]+Beta (y[ [i] ]-Mu), \\ $\{i$, Length $[y]\}]$ \\ Clear [Alpha, Beta, Delta, Mu ] \\ $\operatorname{Do}[\operatorname{ND}[j]=\{$ Mean $[\operatorname{asset}[j]]$, \\ StandardDeviationMLE[asset [j] ] \}, \\ $\{j$, Dimensions [data] [ [2] ] \}] \\ Do [res $[j]=$ NMaximize [ $\{$ Lasset $[j]$, Alpha,Beta, \\ Delta, Mu], Abs[Beta]<Alpha \&\&
}


Delta $>0 \& \&$ Alpha $>5\},\{$ Alpha, Beta, Delta, Mu,

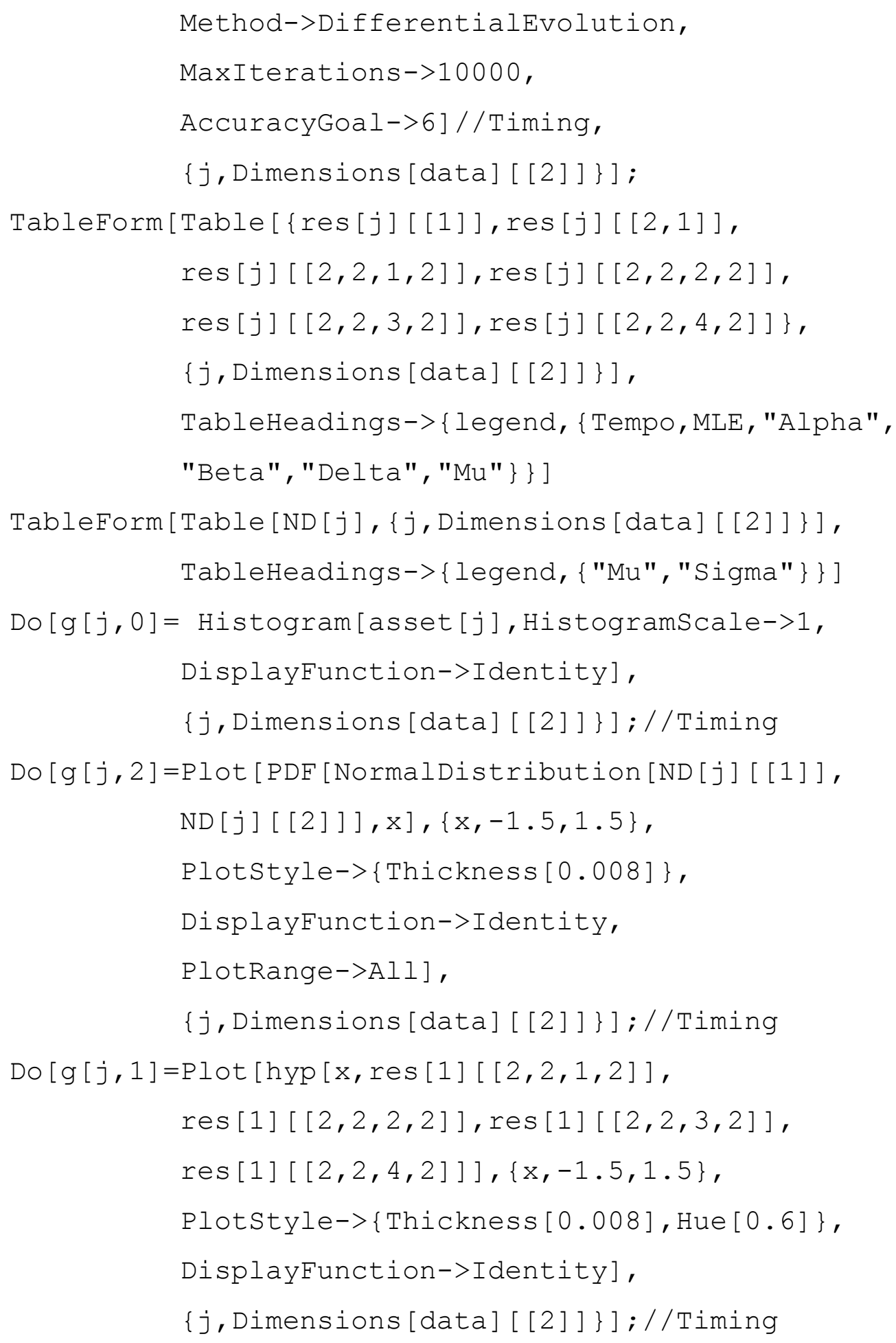




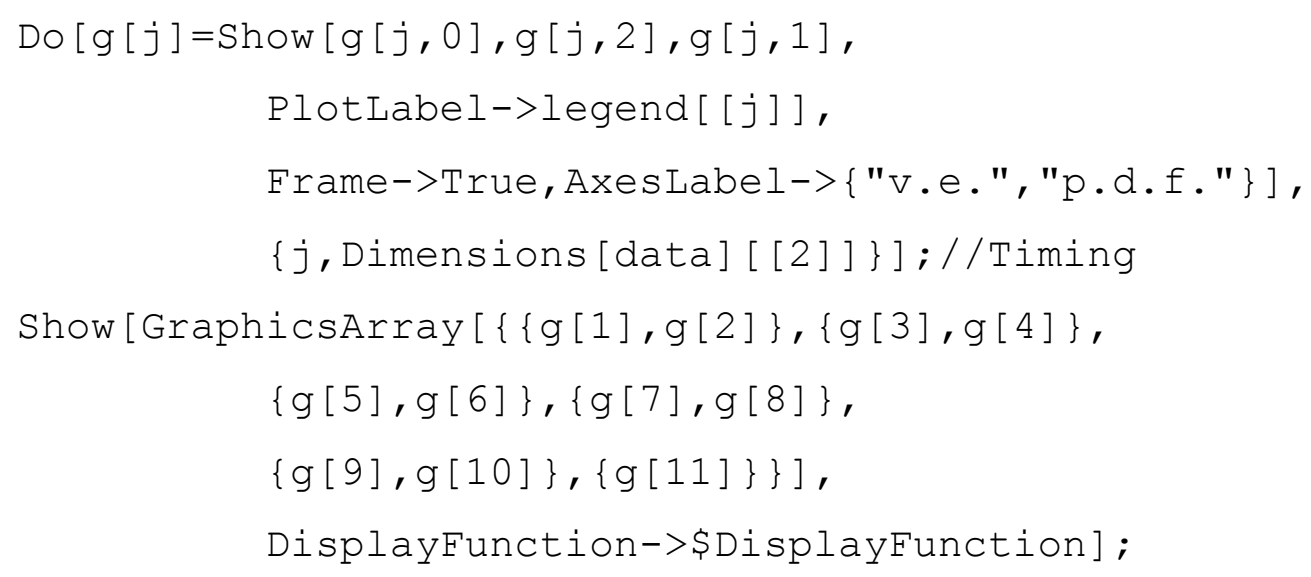

\section{A.2 Algoritmo referente ao Quadro 4.1}

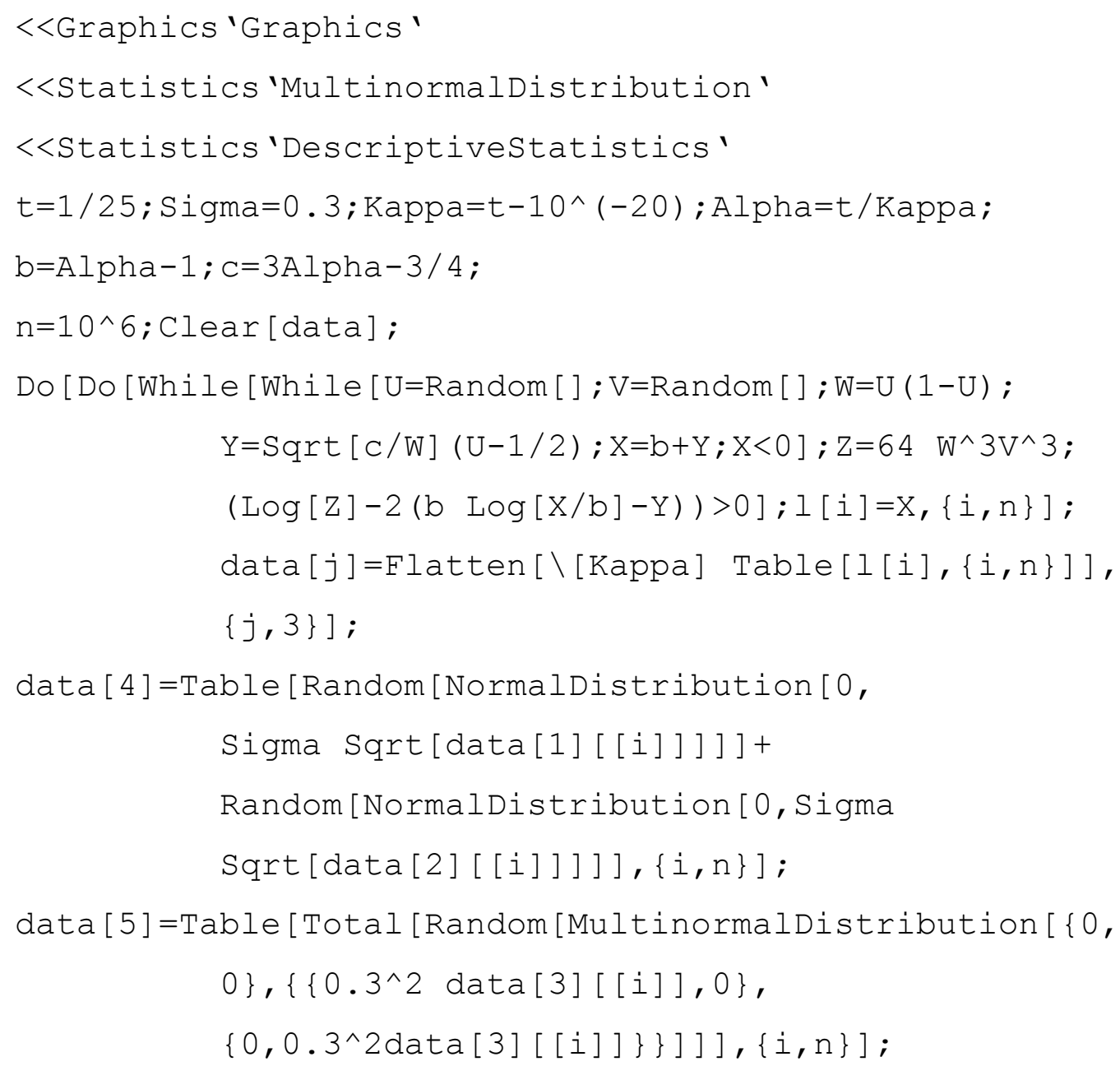




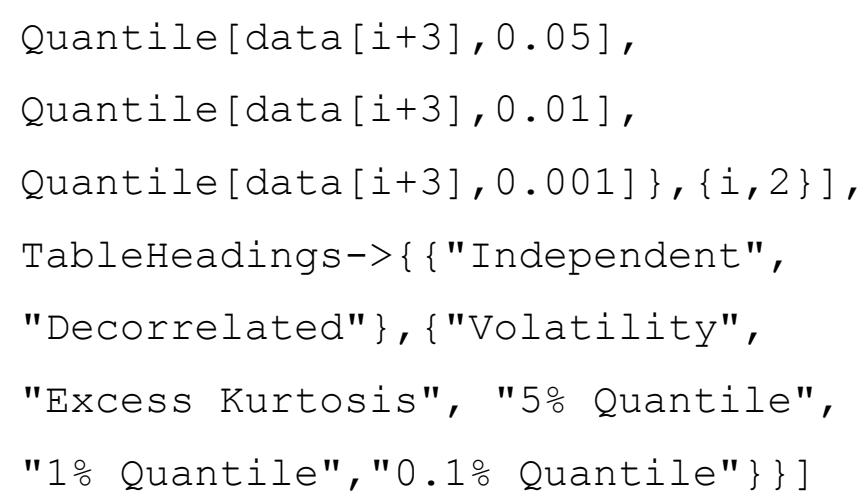

\section{A.3 Algoritmo referente à Figura 0.1}

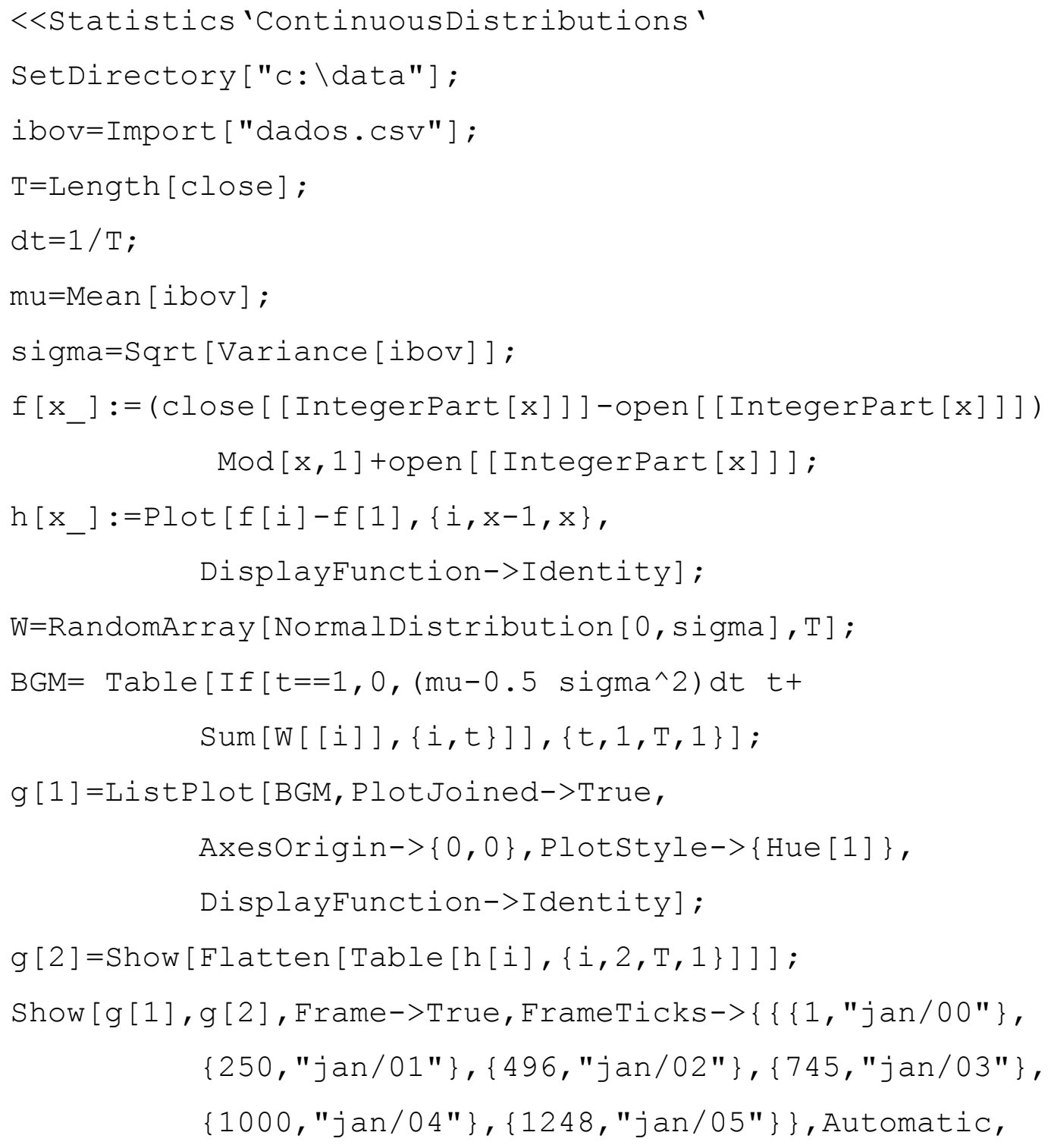




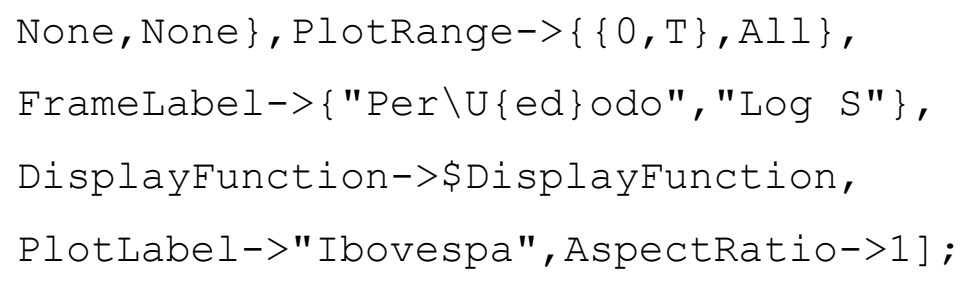

\section{A.4 Algoritmo referente à Figura 0.2}

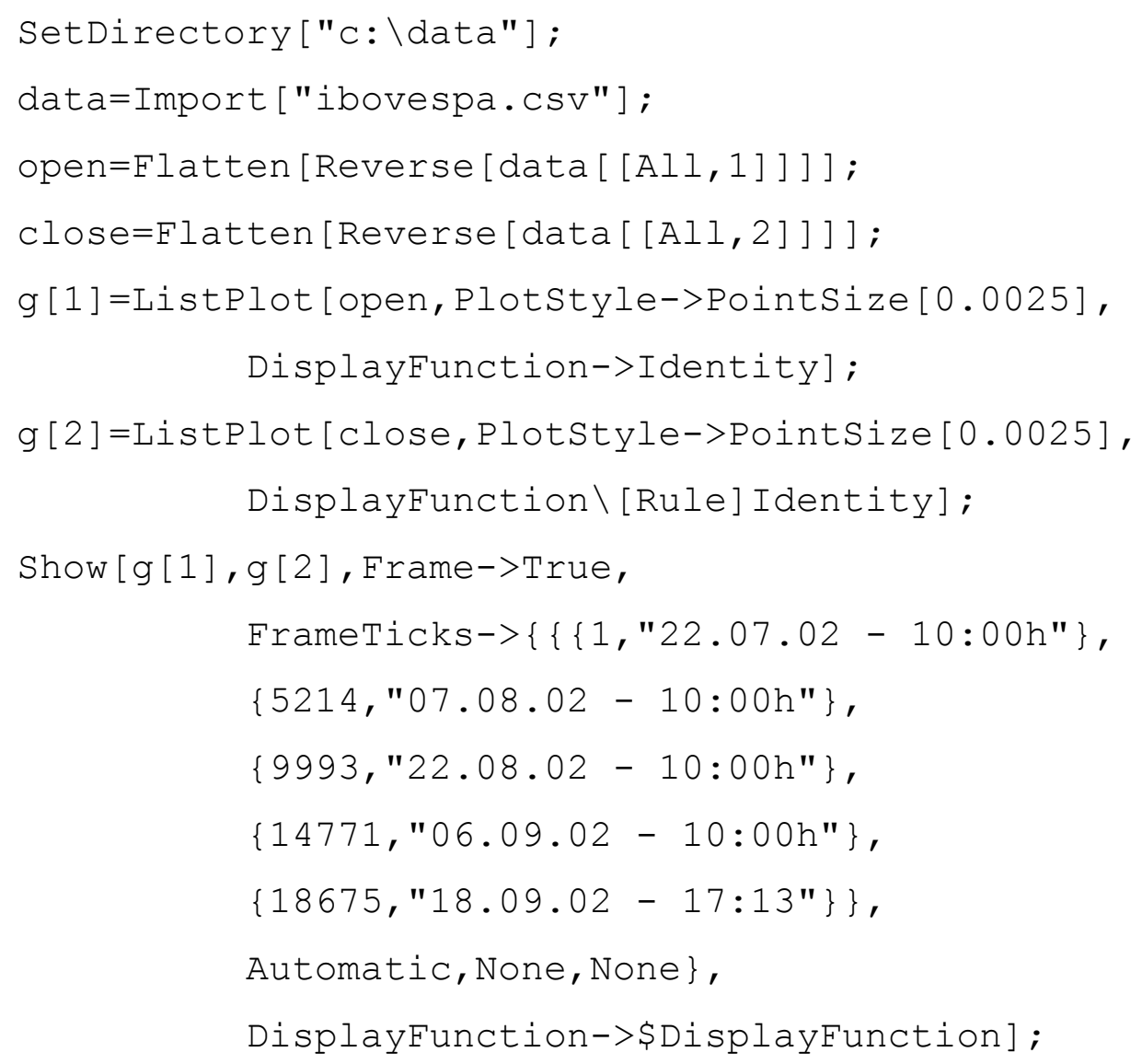

\section{A.5 Algoritmo referente à Figura 1.1}

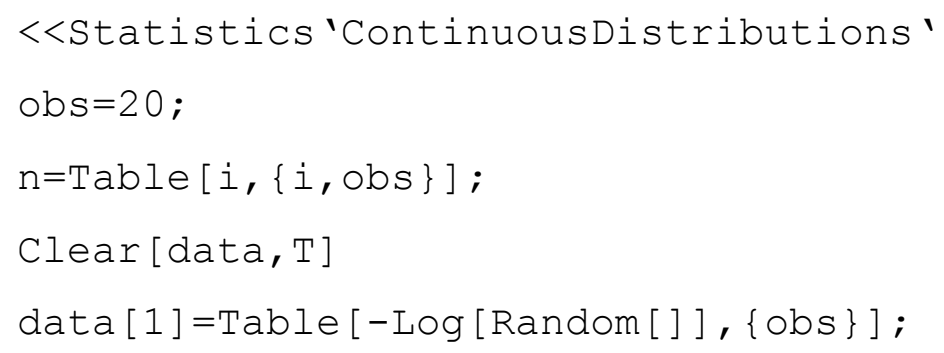


$\mathrm{T}[1]=\mathrm{Tab} l e[\operatorname{Sum}[\operatorname{data}[1][[i]],\{i, j\}],\{j, \mathrm{obs}\}]$;

g[1]=Graphics $[\{$ Thickness $[0.005], \operatorname{Line}[\{\{\mathrm{T}[1][[1]], 0\}$, $\{0,0\}\}]\}]$;

$g[2]=$ Graphics $[\{$ Dashing $[\{0.005\}], \operatorname{Line}[\{\{\mathrm{T}[1][[1]], 0\}$, $\{\mathrm{T}[1][[1]], \mathrm{n}[[1]]\}\}]\}]$;

g [3] =Table [Graphics [ Thickness [0.005], Line [\{ \{ T [1] [ [i] ], $n[[i-1]]\},\{T[1][[i-1]], n[[i-1]]\}\}]\}]$, $\{i, 2, o b s\}]$;

g[4] =Table [Graphics [ Dashing [ $\{0.005\}]$, Line [\{ $\{\mathrm{T}[1][[i]]$, $\mathrm{n}[[\mathrm{i}-1]]\},\{\mathrm{T}[1][[\mathrm{i}]], \mathrm{n}[[\mathrm{i}]]\}\}]\}],\{i, 2, \mathrm{obs}-1\}]$; data $[2]=$ Table $[-\log [$ Random []$],\{$ obs $\}]$; $\mathrm{T}[2]=\mathrm{Table}[\operatorname{Sum}[\operatorname{data}[2][[i]],\{i, j\}],\{j, \mathrm{obs}\}]$; g [5] =Graphics [ $\{$ Thickness [0.005], Hue [1], Line $[\{\{\mathrm{T}[2][[1]], 0\},\{0,0\}\}]\}]$; g[6] =Graphics $[\{$ Dashing $[\{0.005\}], \operatorname{Line}[\{\{\mathrm{T}[2][[1]], 0\}$, $\{\mathrm{T}[2][[1]], \mathrm{n}[[1]]\}\}]\}]$; g[7]=Table [Graphics [ $\{$ Thickness [0.005], Hue [1], Line $[\{\{\mathrm{T}[2][[i]], \mathrm{n}[[i-1]]\},\{\mathrm{T}[2][[i-1]]$, $n[[i-1]]\}\}]\}],\{i, 2$, obs $\}]$; g [8]=Table [Graphics [ $\{$ Dashing $[\{0.005\}]$, Line $[\{\{\mathrm{T}[2][[i]], \mathrm{n}[[i-1]]\}$, $\{\mathrm{T}[2][[\mathrm{i}]], \mathrm{n}[[\mathrm{i}]]\}\}]\}],\{\mathrm{i}, 2, \mathrm{obs}-1\}]$; $g[0]=\operatorname{Graphics}[\operatorname{Line}[\{\{0,0\},\{\operatorname{Max}[\mathrm{T}[1][[\mathrm{obs}]]$, $\mathrm{T}[2][[\mathrm{obs}]]], 0\}\}]]$; Show $[g[0], g[1], g[2], g[3], g[4], g[5], g[6], g[7], g[8]$, Frame->True,FrameLabel->\{"Tempo", "Contagem" , AspectRatio->0.65];

\section{A.6 Algoritimo referente à Figura 1.2}

$<<$ Statistics 'ContinuousDistributions' 


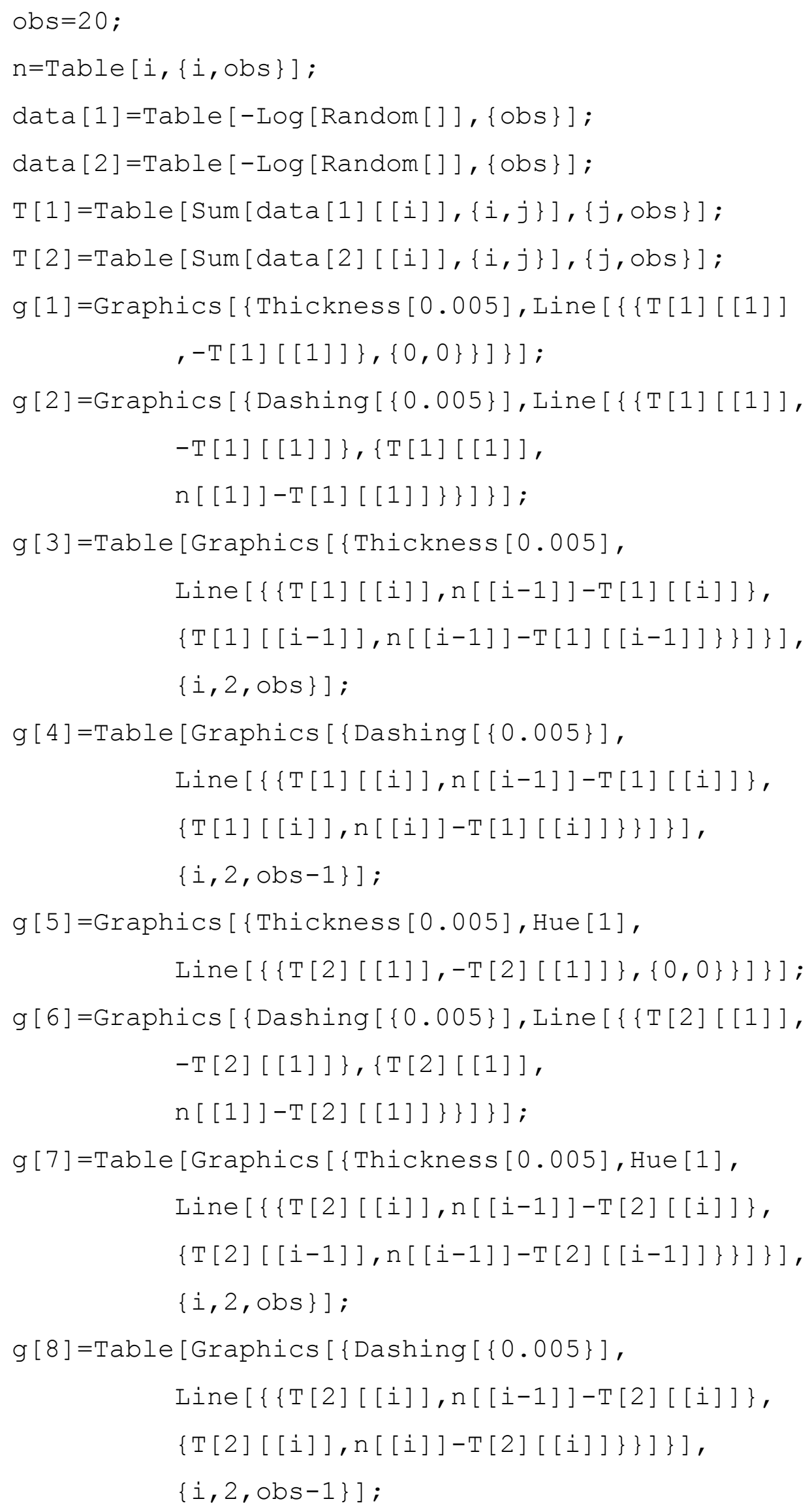


$g[0]=\operatorname{Graphics}[\operatorname{Line}[\{\{0,0\},\{\operatorname{Max}[\mathrm{T}[1][[\mathrm{obs}]]$,

$\mathrm{T}[2][[\mathrm{obs}]]], 0\}\}]]$;

Show $[g[0], g[1], g[2], g[3], g[4], g[5], g[6], g[7], g[8]$,

Frame->True, FrameLabel->\{"Tempo", "Contagem"\}, AspectRatio->0.65];

\section{A.7 Algoritmo referente à Figura 2.1}

$<<$ Statistics 'ContinuousDistributions'

$<<$ Statistics 'DiscreteDistributions'

Clear [obs, n]

obs [1] =Random [PoissonDistribution [10] ];

z [1] =RandomArray [NormalDistribution [0,1], obs [1] ] ;

$n[1]=\operatorname{Tab} l e[\operatorname{Sum}[z[1][[j]],\{j, i\}],\{i$, obs $[1]\}]$;

obs [2] =Random [PoissonDistribution [10] ] ;

z [2] =RandomArray [NormalDistribution [0,1], obs [2] ] ;

$n[2]=$ Table $[\operatorname{Sum}[z[2][[j]],\{j, i\}],\{i$, obs [2] $\}]$;

Clear [data, $\mathrm{T}$ ]

data $[1]=$ Table $[-\log [\operatorname{Random}[]],\{$ obs [1] $\}$;

$\mathrm{T}[1]=\mathrm{Tab} l e[\operatorname{Sum}[\operatorname{data}[1][[i]],\{i, j\}],\{j, \mathrm{obs}[1]\}]$;

$g[1]=$ Graphics $[\{$ Thickness $[0.005]$, Line $[\{\{\mathrm{T}[1][[1]], 0\}$,

$\{0,0\}\}]\}]$;

$g[2]=\operatorname{Graphics}[\{$ Dashing $[\{0.005\}], \operatorname{Line}[\{\{\mathrm{T}[1][[1]], 0\}$,

$\{\mathrm{T}[1][[1]], \mathrm{n}[1][[1]]\}\}\}] ;$

g[3]=Table [Graphics [ Thickness [0.005], Line [\{ \{ T [1] [ [i] ],

$n[1][[i-1]]\},\{\mathrm{T}[1][[i-1]], \mathrm{n}[1][[i-1]]\}\}]\}]$,

$\{i, 2$,obs [1] $\}$;

g [4]=Table [Graphics [ Dashing [ $\{0.005\}]$, Line [\{ $\{\mathrm{T}[1][[\mathrm{i}]]$,

$n[1][[i-1]]\},\{\mathrm{T}[1][[i]], \mathrm{n}[1][[i]\}\}]\}]$,

$\{i, 2$,obs [1]-1\}]; data [2]=Table [-Log [Random[] ], $\{$ obs [2] \}]; 
$\mathrm{T}[2]=\mathrm{Tab} l e[\operatorname{Sum}[\operatorname{data}[2][[i]],\{i, j\}],\{j, \mathrm{obs}[2]\}]$; g [5] =Graphics [ Thickness [0.005], Hue [1], Line [\{ T [2] [ [1] ],0\}, $\{0,0\}\}]\}]$;

g[6]=Graphics $[\{$ Dashing $[\{0.005\}]$, Line $[\{\{\mathrm{T}[2][[1]], 0\}$, $\{\mathrm{T}[2][[1]], \mathrm{n}[2][[1]\}\}]\}] ;$ g[7] =Table [Graphics [ Thickness [0.005], Hue [1], Line $[\{\{[2][[i]], n[2][[i-1]]\},\{T[2][[i-1]]$, $n[2][[i-1]]\}\}]\}],\{i, 2$, obs [2] $\}]$;

g[8]=Table [Graphics [ $\{$ Dashing $[\{0.005\}]$, Line $[\{\{\mathrm{T}[2][[i]]$,

$n[2][[i-1]]\},\{\mathrm{T}[2][[i]], \mathrm{n}[2][[i]]\}\}]\}]$,

$\{i, 2$, obs $[2]-1\}]$; g[0]=Graphics $[\operatorname{Line}[\{\{0,0\},\{\operatorname{Max}[\mathrm{T}[1][[\mathrm{obs}[1]]]$, $\mathrm{T}[2][[\mathrm{obs}[2]]]], 0\}\}]]$; Show $[g[0], g[1], g[2], g[3], g[4], g[5], g[6], g[7], g[8]$, Frame->True, FrameLabel->\{"Tempo", "Contagem"\}, AspectRatio->0.65];

\section{A.8 Algoritmo referente à Figura 2.2}

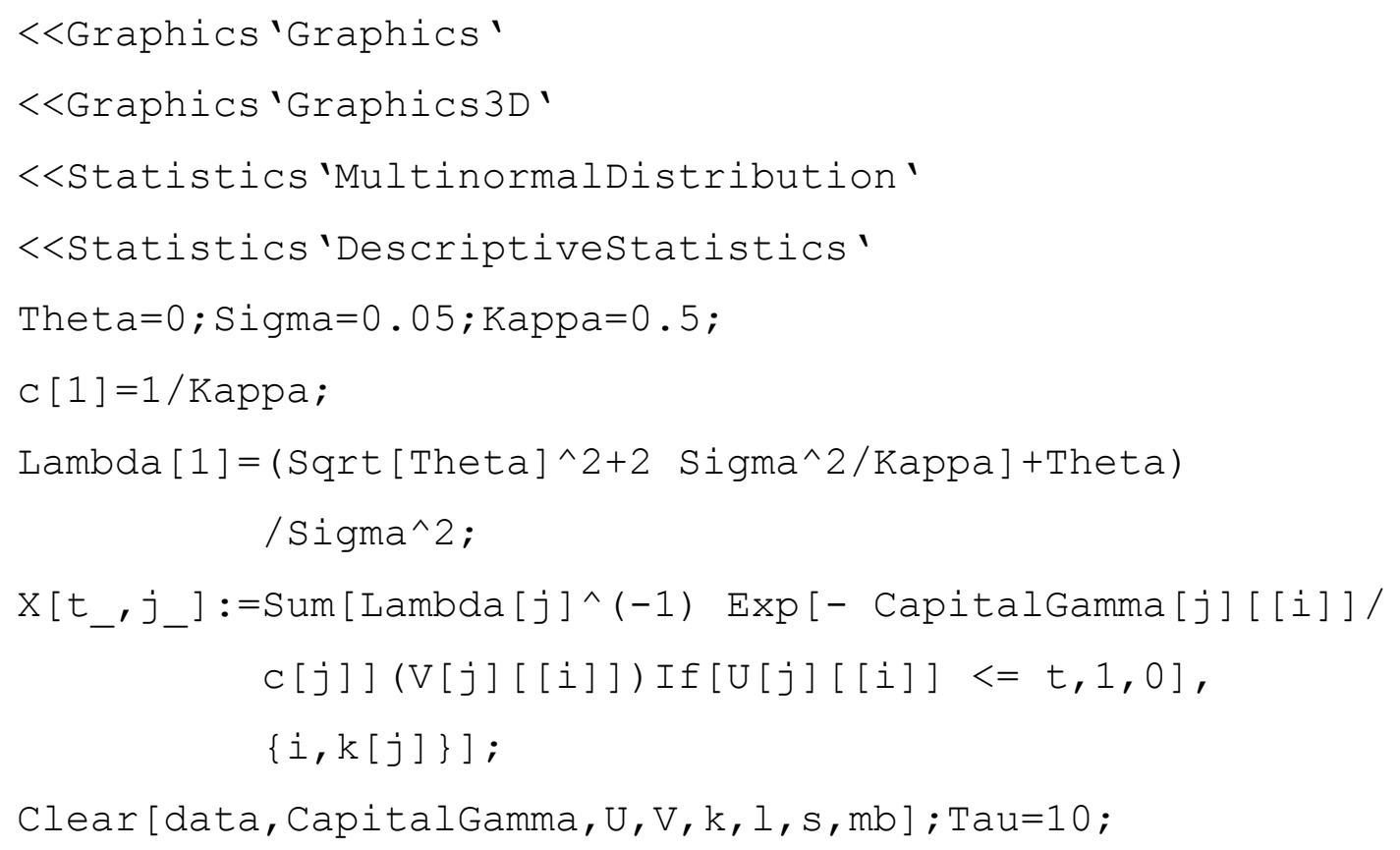


$\mathrm{Do}[\mathrm{mb}[0]=0$;

$m b[s]=m b\left[s-10^{\wedge}(-6)\right]+$ Random [NormalDistribution [s Theta,

Sqrt [s] Sigma] ] /10^3, $\left.\left\{\mathrm{s}, 10^{\wedge}(-6), 1,10^{\wedge}(-6)\right\}\right]$;

DATA $[0]=$ Table $\left[\{\mathrm{s}, \operatorname{mb}[\mathrm{s}]\},\left\{\mathrm{s}, 0,1,10^{\wedge}(-6)\right\}\right]$;

g[0]=ListPlot [DATA [0],PlotStyle->PointSize[0.001],

Frame->True, DisplayFunction->Identity] ;

Do $[\mathrm{mb}[0]=0$;

$m b[s]=m b\left[s-10^{\wedge}(-6)\right]$ +Random [NormalDistribution [s Theta,

Sqrt $\left.[\mathrm{s}] \mathrm{Sigma}]] / 10^{\wedge} 3,\left\{\mathrm{~s}, 10^{\wedge}(-6), 1,10^{\wedge}(-6)\right\}\right]$;

$\operatorname{DATA}[1]=\mathrm{Tab} l e\left[\{\mathrm{~s}, \mathrm{mb}[\mathrm{s}]\},\left\{\mathrm{s}, 0,1,10^{\wedge}(-6)\right\}\right]$;

g[1]=ListPlot [DATA [1],PlotStyle->PointSize[0.001],

Frame->True, DisplayFunction->Identity] ;

Clear $[\mathrm{s}]$; Do $[1[j][0]=0 ; \mathrm{s}=0$;

While $[s=s+1 ;(1[j][s]=1[j][s-1]-\log [\operatorname{Random}[]])<\operatorname{Tau}]$;

$\mathrm{k}[j]=s-1$; CapitalGamma [j]=Table [1 [j] [i],

$\{i, k[j]\}] ; U[j]=T a b l e[R a n d o m[],\{k[j]\}] ;$

$\mathrm{V}[j]=\mathrm{Table}[\operatorname{Sum}[-\log [\operatorname{Random}[]],\{z\}],\{\mathrm{z}, \mathrm{k}[j]\}]$;

data $[j]=$ Table $\left[x[i, j],\left\{i, 0,1,10^{\wedge}(-6)\right\}\right]$,

$\{j, 1\}]$;

DATA $[2]=$ Table $\left[\left\{i, \operatorname{data}[1]\left[\left[\left(i+10^{\wedge}(-6)\right) 1^{\wedge} 6\right]\right]\right\}\right.$,

$\left.\left\{i, 0,1,10^{\wedge}(-6)\right\}\right]$;

g[2]=ListPlot [DATA [2],PlotStyle->PointSize[0.005],

PlotRange->All, Frame->True,

DisplayFunction->Identity] ;

$\operatorname{DATA}[3]=\operatorname{Table}\left[\left\{i, \operatorname{data}[1]\left[\left[\left(i+10^{\wedge}(-6)\right) 1^{\wedge} 6\right]\right]+\operatorname{mb}[i]\right\}\right.$,

$\left.\left\{i, 0,1,10^{\wedge}(-6)\right\}\right] ; / /$ Timing

g[3]=ListPlot [DATA [3],PlotStyle->PointSize[0.001],

PlotRange->All, Frame->True,

DisplayFunction->Identity] ;

Show [GraphicsArray $[\{\{g[0]\},\{g[3]\}\}]$,

DisplayFunction->\$DisplayFunction]; 


\section{A.9 Algoritmo referente à Figura 4.1}

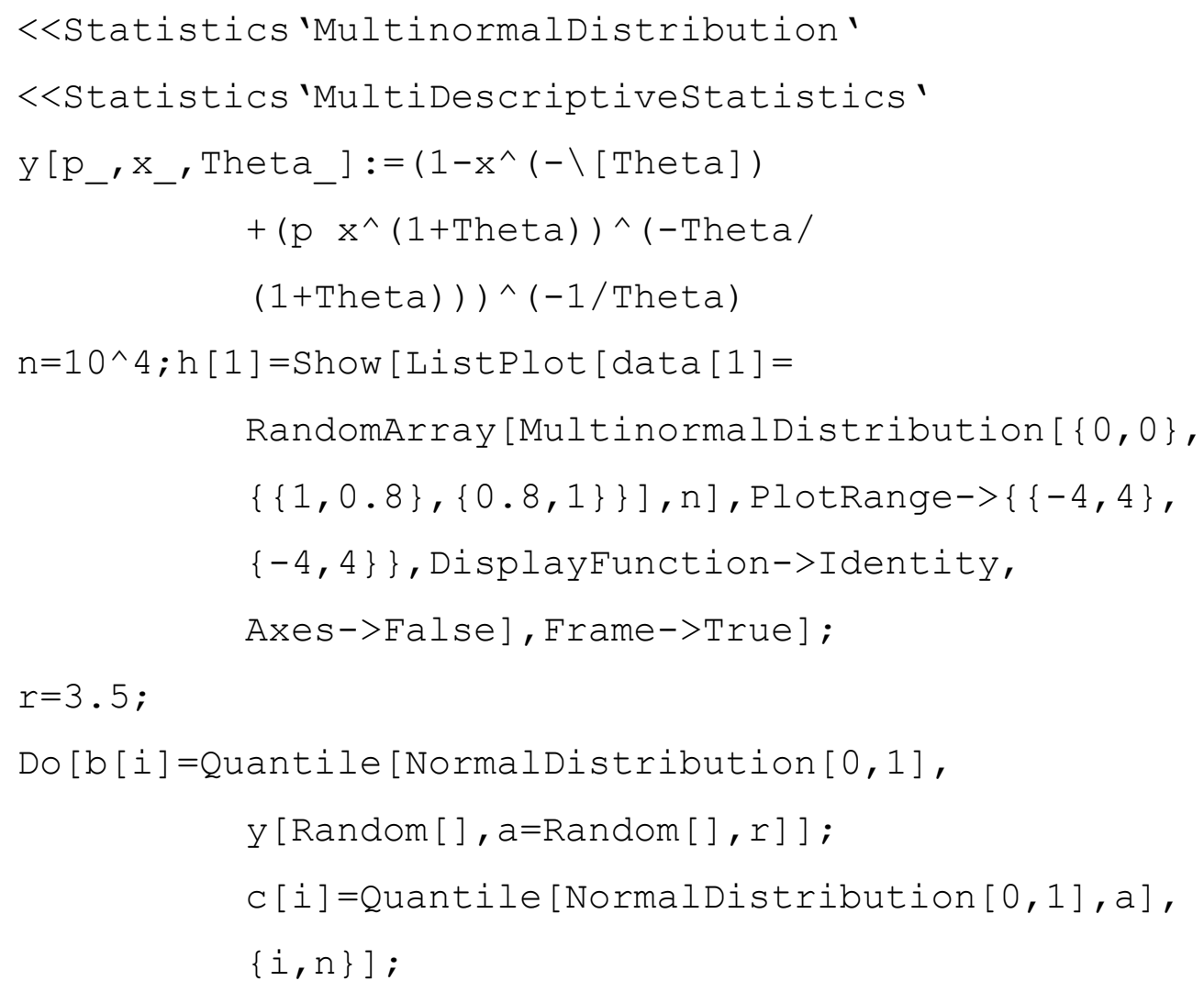


Show [GraphicsArray $[\{\{h[1], h[2]\},\{h[3], h[4]\}\}$,

GraphicsSpacing->\{0,0\}],

DisplayFunction->\$DisplayFunction] ;

\section{A.10Algoritmo referente à Figura 4.2}

Clear [Lambda, c ] ;

$\mathrm{Nu}\left[\mathrm{C}_{-}\right.$, Lambda_, $\left.\mathrm{x}_{-}\right]:=\mathrm{C} \operatorname{Exp}[-$ Lambda $\mathrm{x}] / \mathrm{x}$

$\mathrm{F}\left[\mathrm{v}_{-}, \mathrm{u}_{-}, \mathrm{Xi} \mathrm{i}_{-}\right]:=(1+(\mathrm{u} / \mathrm{v}) \wedge \mathrm{Xi})^{\wedge}(-1-1 / \mathrm{Xi})$

Finv $\left[y_{-}, u_{-}, \backslash[X i]_{-}\right]:=u\left(y^{\wedge}(-X i)\right.$

$(1+\backslash[\mathrm{Xi}]))-1)^{\wedge}(-1 / \mathrm{Xi})$

Tau=10^3; Theta=-0.1; Sigma=0.3; Kappa=0.5；

$\mathrm{Xi}[1]=1 ; \mathrm{Xi}[2]=5$;

c [1]=1/Kappa; c [2]=1/Kappa;

Lambda[1] =(Sqrt[Theta^2+2 Sigma^2/Kappa]

-Theta)/Sigma^2;

Lambda [2] $=($ Sqrt [Theta^2+2 Sigma^2/Kappa ]

+Theta)/Sigma^2;

Clear[Nuint, h]

Do [Nuint $[j]=$ FunctionInterpolation [Integrate [Nu [c [j],

Lambda $[j], x],\{x, y, 5\}],\left\{y, 10^{\wedge}(-10), 5\right\}$,

MaxRecursion->200, Interpolationorder->2],

$\{j, 2\}]$;

$h\left[x_{-}, j_{-}\right]:=\operatorname{If}\left[x>5,5, \operatorname{If}\left[x<10^{\wedge}(-16), 10^{\wedge}(-16)\right.\right.$,

Nuint $[j][\mathrm{x}]]]$

Uinv [Gamma_, j_] :=If $[$ Gamma $>32$, FindRoot $[\mathrm{h}[\mathrm{x}, \mathrm{j}]==32$,

$\{x, 1\}][[1,2]]$, FindRoot $[h[x, j]==$ Gamma,

$\{x, 1\}][[1,2]]]$

$1[0]=0 ; k=0 ;$ While $[k=k+1 ;(1[k]=1[k-1]$

-Log [Random [ ] ] ) $<$ Tau ] ; k=k-1;

CapitalGamma $[1]=$ Table $[1[\mathrm{i}],\{\mathrm{i}, \mathrm{k}\}]$; 
CapitalGamma [2]=Finv [Random [], CapitalGamma [1],

$$
\mathrm{Xi}[1]] ;
$$

$1[0]=0 ; k=0 ;$ While $[\mathrm{k}=\mathrm{k}+1 ;(1[\mathrm{k}]=1[\mathrm{k}-1]$

-Log [Random [ ] ] ) $<\mathrm{Tau}] ; \mathrm{k}=\mathrm{k}-1$;

CapitalGamma [3]=Table $[1[\mathrm{i}],\{\mathrm{i}, \mathrm{k}\}]$;

CapitalGamma [4]=Finv [Random [],

CapitalGamma [3],Xi[2]];

Do [UInv [j] =Table [Uinv [CapitalGamma [j] [ [i] ],

If $[j>2,2,1]]$,

$\{i$, Length [CapitalGamma $[j]]\}],\{j, 4\}]$

Do $[V[j]=$ Table [Random [ ],

$\{$ Length [CapitalGamma $[j+1]]\}],\{j, 2\}]$;

$\mathrm{X}\left[\mathrm{s}_{-}, j_{-}\right]:=\operatorname{Sum}[\mathrm{UInv}[j][[i]] \operatorname{If}[\mathrm{V}[1][[i]]<\mathrm{s}, 1,0]$,

$\{i$, Length [UInv [j] ] \} ]

$Y\left[s_{-}, j_{-}\right]:=\operatorname{Sum}[\operatorname{UInv}[j+2][[i]] \operatorname{If}[\mathrm{V}[2][[i]]<s, 1,0]$,

$\{i, \operatorname{Length}[\mathrm{UInv}[j+2]]\}]$

Do $[g[j]=L i s t P l o t[T a b l e[\{i, X[i, j]-Y[i, j]\}$,

$\left.\left\{i, 0,1,10^{\wedge}(-2)\right\}\right]$,

PlotStyle->\{Thickness [0.005], If [j==2, Hue [1],

GrayLevel[0] ] \},PlotJoined->True,

DisplayFunction->Identity], $\{j, 2\}]$;

Show $[g[1], g[2]$, DisplayFunction->\$DisplayFunction,

Frame->True];

\section{A.11Algoritmo referente à Figura 4.3}

Clear [c, Theta, Xi]

$\mathrm{Nu}\left[\mathrm{C}_{-}\right.$, Lambda_, $\left.\mathrm{x}_{-}\right]:=\mathrm{C} \operatorname{Exp}[-$ Lambda $\mathrm{x}] / \mathrm{x}$

Finv $\left[y_{-}, u_{-}\right.$, Theta_ $]:=\left(-u^{\wedge}\right.$ Theta+ $\left(-u^{\wedge}(1-\text { Theta }) \quad y\right)^{\wedge}(-$ Theta

$(-1+\backslash$ Theta $)))^{\wedge}(1 /$ Theta $)$

$\mathrm{Tau}=10^{\wedge} 2 ; \mathrm{c}=30 ; \mathrm{Lambda}=1 ; \mathrm{Xi}=-3$; 
Uinv[Gamma_] :=If [Gamma>Tau, 0, FindRoot [ Integrate [ $\mathrm{Nu}[\mathrm{c}$, Lambda, $\mathrm{x}],\{\mathrm{x}, \mathrm{y}, \operatorname{Infinity}\}]==$ Gamma, $\left.\left.\left\{\mathrm{y}, 10^{\wedge}(-15)\right\}\right][[1,2]]\right]$

$1[0]=0 ; k=0 ;$ While $[k=k+1 ;(1[k]=1[k-1]$

$-\log [$ Random [ ] ] ) $<$ Tau $] ; k=k-1$;

CapitalGamma [1] =Table $[1[\mathrm{i}],\{\mathrm{i}, \mathrm{k}\}]$;

CapitalGamma [2]=Re [Finv[Random [],

CapitalGamma[1],Xi]];

$1[0]=0 ; k=0 ;$ While $[k=k+1 ;(1[k]=1[k-1]$

$-\log [$ Random [ ] ] ) $<$ Tau ] ; k=k-1;

CapitalGamma [3] =Table [l[i], \{i,k\}] ;

CapitalGamma [4]=Re [Finv[Random[],

CapitalGamma[3],Xi]];

Do[UInv[j]=Table [Uinv[CapitalGamma[j] [ [i] ] ],

$\{i$, Length [CapitalGamma $[j]]\}],\{j, 4\}]$

Do $[V[j]=$ Table [Random [ ],

$\{$ Length [CapitalGamma $[j+1]]\}],\{j, 2\}]$;

$\mathrm{X}\left[\mathrm{s}_{-}, j_{-}\right]:=\operatorname{Sum}[\mathrm{UInv}[j][[i]] \operatorname{If}[\mathrm{V}[1][[i]]<\mathrm{s}, 1,0]$,

$\{i$, Length [UInv $[j]]\}]$

$Y\left[s_{-}, j_{-}\right]:=\operatorname{Sum}[\operatorname{UInv}[j+2][[i]] \operatorname{If}[\mathrm{V}[2][[i]]<s, 1,0]$,

$\{i, \operatorname{Length}[\mathrm{UInv}[j+2]]\}]$

g[1]=ListPlot $[$ Table $[\{i, X[i, 1]-Y[i, 1]\}$,

$\left.\left\{i, 0,1,10^{\wedge}(-3)\right\}\right]$,

PlotStyle->\{Thickness [0.005], Hue [1]\},

PlotJoined->True, Frame->True,

Displayfunction->Identity] ;

$g[2]=L i s t P l o t[$ Table $[\{i,-X[i, 2]+Y[i, 2]\}$,

$\left.\left\{i, 0,1,10^{\wedge}(-3)\right\}\right]$,

Plotstyle->\{Thickness[0.005],

GrayLevel[0] \},PlotJoined->True,

Frame->True, DisplayFunction->Identity] ;

Show [g[1],g[2], DisplayFunction->\$DisplayFunction] ; 


\section{Apêndice B \\ Teoria da Medida e Integração}

\section{B.1 $\sigma$-álgebra e Medida}

A noção de medida pode ser entendida como uma generalização de conceitos conhecidos como distância, área e volume. Considere, por exemplo, um conjunto $E$, o qual é definido em $\mathbb{R}^{d}$, ou em alguma função de valor definido em $\mathbb{R}^{d}$. A medida $\mu$ definida em $E$ associa a certos subconjuntos $A \subset E$, que são ditos conjuntos mensuráveis, um número $\mu(A) \in[0 ; \infty]$ denominado medida de $A$. Por analogia à idéia de volume é natural dizer que um conjunto vazio $\varnothing$ tem medida nula, i.e., $\mu(\varnothing)=0$. Adicionalmente, se $A$ e $B$ são conjutos mensuráveis desconectados, i.e., $A \cap B=\varnothing$, então, $A \cup B$ deve obrigatoriamente ser mensurável, tal que sua medida apresenta a propriedade de aditividade, ou seja, ela é igual a $\mu(A \cup B)=\mu(A)+\mu(B)$. De forma geral, uma seqüência finita de subconjuntos mensuráveis desconectados $\left(A_{n}\right)_{n \in \mathbb{N}}$ apresenta a seguinte propriedade:

$$
\mu\left(\bigcup_{n \geq 1} A_{n}\right)=\sum_{n \geq 1} \mu\left(A_{n}\right) .
$$

Se $\mu(E)<\infty$, então, para qualquer conjunto mensurável $A$ e seu complemento $A^{c}$ também mensurável, i.e. $A \cup A^{c}$, a propriedade de aditividade pode ser aplicada ao seu complemento como

$$
\mu\left(A^{c}\right)=\mu(E)-\mu(A) .
$$

Assim, é possível resumir o conceito de medida dizendo que o domínio de uma medida definida no conjunto mensurável $E$ é composto por uma coleção de subconjuntos de $E$, tal que a coleção de subconjuntos $\mathcal{E}$

1. contenha o conjunto vazio: $\varnothing \in \mathcal{E}$.

2. é estável nas operações de união:

$$
A_{n} \in \mathcal{E},\left(A_{n}\right)_{n \geq 1} \text { desconectados } \Rightarrow \bigcup_{n \geq 1} A_{n} \in \mathcal{E}
$$

3. contenha o complemento de todos os elementos: $\forall A \in \mathcal{E}, A^{c} \in \mathcal{E}$. 
A coleção de subconjuntos $\mathcal{E}$ é denominado $\sigma$-álgebra. Será dotado a fonte caligrafia para denominar $\sigma$-álgebras, e.g., $\mathcal{E}, \mathcal{B}$ ou $\mathcal{F}$. Um conjunto mensurável será, desta maneira, um elemento da $\sigma$-álgebra. Para construir uma $\sigma$-álgebra é necessário identificar a coleção de conjuntos que se quer medir, e.g., intervalos definidos nos reais. Depois disto é necessário formar os conjuntos obtidos pela união e complementariedade e obter a $\sigma$-álgebra desejada.

Proposição B.1 Dada uma coleção $\mathcal{A}$ de subconjuntos de E, então, existirá uma única $\sigma$-álgebra denominada $\sigma(\mathcal{A})$ que apresentará a seguinte propriedade: se qualquer $\sigma$-álgebra $\mathcal{F}$ contém $\mathcal{A}$, então, $\sigma(\mathcal{A}) \subset \mathcal{F}$. $\sigma(\mathcal{A})$ é a menor $\sigma$-álgebra que contém $\mathcal{A}$ e é dita $\sigma$-álgebra gerada por $\mathcal{A}$.

Um exemplo da Proposição B.1 é quando $E=\mathbb{R}^{d}$ é uma espaço topológico que é composto por intervalos abertos e fechados. Seja a coleção de todos os intervalos abertos $\mathcal{E} \in E$, a $\sigma$-álgebra gerada por $\mathcal{E}$ é denominada $\sigma(\mathcal{E})$. Se $\mathcal{E} \subset \sigma(E)$, então, pela Proposição B.1, $\sigma(\mathcal{E}) \subset \sigma(E)$. A $\sigma$-álgebra gerada por todos os intervalos abertos $\sigma(\mathcal{E})$ é denominada $\sigma$-álgebra de Borel, $\mathcal{B}(E)$, ou simplesmente $\mathcal{B}$. Esta denominação é utilizada, pois através dos operadores união, complemento e vazio, necessários para gerar uma $\sigma$-álgebra, é possível provar que $\sigma(\mathcal{E})=\sigma(E)$. Isto implica dizer que, qualquer elemento $B \in \mathcal{B}$ (denominado conjunto Borel) pode ser representado por intervalos abertos em $\mathcal{B}$, mesmo sendo um intervalo fechado, pois a quantidade de informação de $\sigma(\mathcal{E})$, que considera a $\sigma$-álgebra apenas da coleção dos intervalos abertos, é igual a quantidade de informação de $\sigma(E)$, que contempla a $\sigma$-álgebra referente à coleção dos intervalos abertos e dos fechados. Medidas definidas em $\mathcal{B}$ irão garantir que todos os conjuntos abertos e fechados sejam mensuráveis.

Definição B.1 Medida Seja $\mathcal{E}$ uma $\sigma$-álgebra de subconjuntos de $E$ e $(E, \mathcal{E})$ denominado espaço mensurável. Uma medida (positiva) em $(E, \mathcal{E})$ é definida como uma função

$$
\begin{aligned}
\mu: \mathcal{E} & \longrightarrow[0 ; \infty] \\
A & \longmapsto \mu(A),
\end{aligned}
$$

sendo que: 
1. $\mu(\varnothing)=0$.

2. Para qualquer seqüência de conjuntos desconectados $A_{n} \in \mathcal{E}$ vale a propriedade aditiva, i.e.

$$
\mu\left(\bigcup_{n \geq 1} A_{n}\right)=\sum_{n \geq 1} \mu\left(A_{n}\right)
$$

Um elemento $A \in \mathcal{E}$ é dito um conjunto mensurável e $\mu(A)$ é a sua medida.

Nesta definição foi indicado que $\mu(A)$ é uma medida positiva. Entretanto, existem medidas sinalizadas que apresentam partes positivas e partes negativas. Se $\mu_{+} \mathrm{e}$ $\mu_{-}$são, respectivamente, as partes positiva e negativa da medida sinalizada $\mu$, então, a propriedade apresentada na Definição B.1 vale para $\mu=\mu_{+}-\mu_{-}$. Uma aplicação importante da Definição B.1 é a medida de Lebesgue em espaço $\mathbb{R}^{d}$. Esta medida é definida na $\sigma$-álgebra de Borel $\mathcal{B}\left(\mathbb{R}^{d}\right)$ e corresponde à idéia do volume de dimensão $d$. De forma geral, para qualquer função contínua positiva $\rho: \mathbb{R}^{d} \longrightarrow \mathbb{R}^{+}$é possível definir uma medida em $\mathbb{R}^{d}$, tal que

$$
\begin{aligned}
\mu: \mathcal{B}\left(\mathbb{R}^{d}\right) & \longrightarrow[0 ; \infty] \\
A & \longmapsto \int_{A} \rho(\lambda) d \lambda=\int_{A} \rho(x) 1_{A} d x .
\end{aligned}
$$

A função $\rho$ é dita densidade de $\mu$ em relação a medida de Lebesgue $\lambda$. A equação (B.4) define uma medida positiva para qualquer função mensurável $\rho$ (funções mensuráveis serão definidas posteriormente).

Medidas de Dirac são também importantes exemplos de medidas. A medida de Dirac $\delta_{x}$ associada a um ponto $x \in E$ é definida como: $\delta_{x}(A)=1$ se $x \in A$ e $\delta_{x}=0$ se $x \notin A$. Dado um conjunto enumerável de pontos $X=\left\{x_{i}, i=0,1,2, \ldots\right\} \subset E$, a medida de contagem $\mu_{X}=\sum_{i} \delta_{x_{i}}$ é definida como: para qualquer $A \subset E, \mu_{X}(A)$ conta-se o número de pontos $x_{i}$ em $A$, tal que

$$
\mu(A)=\sum_{i \geq 1} 1_{\left\{x_{i} \in A\right\}}
$$

Um conjunto mensurável pode ter medida nula sem ser um conjunto vazio. A existência de conjuntos de medida igual a zero é de grande importância para a teoria da medida. Se $A$ é um conjunto mensurável com $\mu(A)=0$, então, é intuitivo dizer que $\mu(B)=0$ para qualquer $B \subset A$. Subconjuntos de conjuntos de medida igual a 
zero são denominados conjuntos nulos (n). Se todos os conjuntos nulos não estiverem contidos em $\mathcal{E}$, então, ao adicioná-los à $\mathcal{E}$ tem-se que a $\sigma$-álgebra é dita completa. Uma medida $\mu$ é dita de valor inteiro, se para qualquer conjunto mensurável $A, \mu(A)$ é um valor inteiro (positivo). Um exemplo de medida de valor inteiro é a medida de Dirac. De uma forma geral, qualquer medida de contagem é uma medida de valor inteiro.

Uma medida $\mu \mathrm{em}(E, \mathcal{E})$ é dita finita se $\mu(\mathcal{E})<\infty$, o que significa que $\mu(A)$ é finita para qualquer conjunto mensurável $A \subset \mathcal{E}$. A quantidade $\mu(\mathcal{E})$ é dita massa de $\mu$. Por exemplo, a medida de Dirac é finita e possui massa igual a 1 . A medida de contagem $\mu_{X}$ em $E$ associada ao conjunto $X=\left\{x_{1}, x_{2}, \ldots\right\}$ é finita se $X$ é finito e possui massa $\mu_{X}(E)$ igual ao número de elementos de $X$.

Uma medida finita com massa igual à 1 é dita medida de probabilidade Neste caso $(E, \mathcal{E}, \mu)$ é dito espaço probabilístico. Nem todas as medidas são medidas finitas. Um exemplo é a medida de Lebesgue em $\mathbb{R}^{d}$, que possui massa total infinita. Um conceito mais flexível é a medida de Radon:

Definição B.2 Medida de Radon Seja $E \subset \mathbb{R}^{d}$. Uma medida de Radon no espaço mensurável $(E, \mathcal{B})$ é uma medida $\mu$, tal que, para qualquer conjunto mensurável compacto (com contorno) $B \subset \mathcal{B}, \mu(B)<\infty$.

A medida de Lebesgue em $\mathbb{R}$ é uma medida de Radon, pois o tamanho de qualquer intervalo é finito. As medidas de Dirac e qualquer combinação linear finita de medidas de Dirac também são medidas de Radon. De uma maneira geral, uma medida $\mu$ em $E \subset \mathbb{R}^{d}$ é dita finita se:

$$
E=\bigcup_{i=1}^{\infty} E_{i} \operatorname{com} \mu\left(E_{i}\right)<\infty .
$$

Esta condição vale quando $\mu$ é uma medida de Radon. As medidas de Lebesgue e Dirac são diferentes em vários aspectos, pois a medida de Dirac é baseada em uma quantidade finita de pontos e a medida de Lebesgue atribui valor zero de massa para um conjunto finito. A medida $\mu_{0}$, que atribui valor zero de massa para qualquer ponto, é dita medida difusa ou ausente de massa, i.e.

$$
\forall x \in E, \mu_{0}(\{x\})=0 .
$$

Medidas de Lebesgue em $\mathbb{R}$ são medidas difusas, pois atribuem massa zero para qualquer ponto individualmente, mas não obrigatoriamente atribuem massa zero para todos 
os intervalos. Medidas de Lebesgue em $\mathbb{R}^{d}$ de densidade contínua são também medidas difusas. Estes resultados mostram que a medida de Radon pode ser decomposta em medidas difusas e em somas de medidas de Dirac.

Proposição B.2 Decomposição de medidas de Radon Qualquer medida de Radon $\mu$ pode ser decomposta na soma de uma medida difusa e combinações lineares de medidas de Dirac:

$$
\mu=\mu_{0}+\sum_{j \geq 1} b_{j} \delta_{x_{j}} \quad x_{j} \in E, b_{j}>0
$$

\section{B.2 Medidas e Funções: Integração}

Considere dois espaços mensuráveis $(E, \mathcal{E})$ e $(F, \mathcal{F})$. Muitas vezes é de interesse avaliar uma medida em um conjunto $A \subset \mathcal{E}$, tal como

$$
f(A)=\{f(x): x \in A\} .
$$

Para uma função $f$ qualquer, não é possível garantir que esta função seja mensurável. Assim, é importante definir o que é uma função mensurável.

Definição B.3 Função mensurável $\operatorname{Sejam~}(E, \mathcal{E}) e(F, \mathcal{F})$ dois espaços mensuráveis. A função $f: E \longrightarrow F$ é dita mensurável se, para qualquer conjunto $A^{\prime} \subset \mathcal{F}$, o conjunto

$$
f^{-1}\left(A^{\prime}\right)=\left\{x: x \in E, f(x) \in A^{\prime}\right\},
$$

sendo que $f^{-1}\left(A^{\prime}\right)$ é um subconjunto (mensurável) de $\mathcal{E}$.

Ao considerar medidas em conjuntos que possuem métricas ou estruturas topológicas, tal como $\mathbb{R}^{d}$ e sua medida euclidiana, obtêm resultados interessantes. Nestes espaços o conceito de continuidade para funções é bem definido e coincide com a Definição B.3, i.e., a imagem de $f^{-1}$ de um conjunto aberto em $F$ é um conjunto aberto em $E$. Isto explica porque o $\sigma$-algebra de Borel $\mathcal{B}$ é um dos conjuntos mais utilizados como $\sigma$-álgebra de espaços mensuráveis. Outros exemplos de funções 
mensuráveis são funções do tipo $f: E \longrightarrow \mathbb{R}$

$$
f=\sum_{j=1}^{n} c_{j} 1_{A_{j}},
$$

sendo que $\left(A_{j}\right)$ são conjuntos mensuráveis e $c_{j} \in \mathbb{R}$. Estes tipos de funções são conhecidas como funções simples. A integral de funções simples em relação a uma medida $\mu$ é definida como

$$
\mu(f)=\sum_{j=1}^{n} c_{j} \mu\left(A_{j}\right) .
$$

É possível estender o conceito de integração para qualquer função mensurável $\mu: E \longrightarrow \mathbb{R}$ ao estabelecer que

$$
\mu(f)=\sup \{\mu(\varphi), \varphi \text { função simples, } \varphi \leq f\} .
$$

A integral $\mu(f)$ pode assumir valor infinito. Assim, qualquer função mensurável $f$ : $E \longrightarrow \mathbb{R}$ pode ser escrita como a soma das suas partes positivas e negativas, i.e., $f=f^{+}-f^{-}$, onde $f, f^{+}$e $f^{-} \geq 0$. Pode-se definir separadamente $\mu\left(f^{+}\right)$e $\mu\left(f^{-}\right)$. Se $\mu\left(f^{+}\right)$e $\mu\left(f^{-}\right)<\infty$, então, $f$ é uma função integrável na medida $\mu$ ( $\mu$-integrável) e, diz-se que $\mu(f)=\mu\left(f^{+}\right)-\mu\left(f^{-}\right)$é a integral $\mu$ em relação a função $f$. Quando $\mu$ é a medida de Lebesgue, então, $\mu(f)$ é a integral de Lebesgue de $f$, que possui representação diferencial

$$
\mu(f)=\int_{x \in E} f(x) \mu(d x) .
$$

Se a medida $\mu$ puder ser decomposta da forma apresentada na Proposição B.2, então, a integral apresentada em (B.11) pode ser interpretada como

$$
\mu(f)=\int f(x) \mu_{0}+\sum_{j \geq 1} b_{j} f\left(x_{j}\right) .
$$

Duas funções mensuráveis $f$ e $g$ em $E$ são consideradas iguais quase em todos os locais em relação a medida $\mu$ ( $\mu$-q.1.) se elas divergirem apenas nos conjuntos nulos:

$$
f=g, \mu \text {-q.1. } \Longleftrightarrow \mu(\{x \in E, f(x) \neq g(x)\})=0 .
$$

Se $f$ e $g$ são $\mu$-integráveis, então,

$$
f=g, \mu \text {-q.1. } \Rightarrow \int_{E} f(x) \mu(d x)=\int_{E} g(x) \mu(d x) .
$$




\section{B.3 Continuidade Absoluta e Densidade}

Sejam as medidas $\mu_{1}$ e $\mu_{2}$ definidas no espaço mensurável $(E, \mathcal{E})$. Para comparar tais medidas é necessário introduzir a definição de continuidade absoluta:

Definição B.4 Continuidade Absoluta A medida $\mu_{2}$ é dita ser absolutamente contínua em relação à medida $\mu_{1}$ se para qualquer conjunto mensurável $A$

$$
\mu_{1}(A)=0 \Rightarrow \mu_{2}(A)=0 .
$$

A Definição B.4 permite estabelecer a seguinte proposição:

Proposição B.3 Teorema de Radon-Nikodym Se $\mu_{2}$ é absolutamente contínua em relação à $\mu_{1}$, então, existe uma função mensurável $Z: E \longrightarrow[0 ; \infty[$ tal que para qualquer conjunto mensurável $A$

$$
\mu_{2}(A)=\int_{A} Z d \mu_{1}=\mu_{1}\left(Z 1_{A}\right) .
$$

A função $Z$ é dita densidade ou derivada de Radon-Nikodym de $\mu_{2}$ em relação $\mu_{1}$, e é representada por $\frac{d \mu_{2}}{d \mu_{1}}$. Para qualquer função $f \mu_{2}$-integrável tem-se que

$$
\mu_{2}(f)=\int_{E} f d \mu_{2}=\mu_{1}(f Z)=\int_{E} f Z d \mu_{1} .
$$

Desta maneira, se $\mu_{2}$ é absolutamente contínua em relação a $\mu_{1}$, então, a integral em relação à $\mu_{2}$ é uma integral ponderada por uma função densidade $Z$ em relação à $\mu_{1}$. Se ambas as medidas são absolutamente contínuas em relação a outra, então, elas são denominadas medidas equivalentes. Quando as medidas $\mu_{1}$ e $\mu_{2}$ são equivalentes, então, necessariamente $Z>0$. 


\section{Apêndice C \\ Teoria de Probabilidade}

\section{C.1 Variável Estocástica e Espaço Probabilístico}

Considere um conjunto $\Omega$, denominado conjunto de cenários, com $\sigma$-álgebra $\mathcal{F}$. No contexto da modelagem financeira, $\Omega$ representa os diferentes cenários de mercado que podem ocorrer, sendo que cada cenário $\omega \in \Omega$ pode ser descrito como a evolução dos preços de diferentes instrumentos financeiros. Uma medida probabilística em $(\Omega, \mathcal{F})$ é uma medida positiva finita $\mathbb{P}$ de massa total igual a $1 .(\Omega, \mathcal{F}, \mathbb{P})$ é denominado espaço probabilístico. Um conjunto mensurável $A \in \mathcal{F}$ é dito evento e é um conjunto de cenários ao qual pode ser atribuída uma probabilidade. Uma medida probabilística atribui uma probabilidade entre 0 e 1 para cada evento:

$$
\begin{aligned}
\mathbb{P}: & \mathcal{F} \longrightarrow[0 ; 1] \\
A & \longmapsto \mathbb{P}(A) .
\end{aligned}
$$

Medidas probabilísticas serão denominadas por letras tais como $\mathbb{P}$ e $\mathbb{Q}$. Um evento $A$ com probabilidade $\mathbb{P}(A)=1$ é dito de ocorrência quase-certa (q.c.). Se $\mathbb{P}(A)=0$, o evento $A$ é entendido como impossível. Ao trabalhar com diversas medidas de probabilidade, definidas no mesmo conjunto mensurável, então, é necessário indicar qual medida de probabilidade está sendo referida. Desta maneira, diz-se $\mathbb{P}$-q.c. ou quasecertamente em $\mathbb{P}$. Um conjunto $\mathbb{P}$-nulo é um subconjunto de um conjunto de eventos impossível. Como foi mencionado anteriomente, é possível completar $\mathcal{F}$ incluindo todos os conjuntos nulos. Isto significa atribuir probabilidade zero para subconjuntos impossíveis, o que parece ser intuitivamente razoável. Neste sentido, é interessante considerar neste estudo $\sigma$-álgebras completas. Ao dizer que uma propriedade ocorre $\mathbb{P}$-q.c., tem-se que o conjunto $\omega \in \Omega$ para qual a propriedade não vale é um conjunto nulo.

Como foi apresentado na Definição B.4 duas medidas probabilísticas $\mathbb{P}$ e $\mathbb{Q}$ em $(\Omega, \mathcal{F})$ são equivalentes (ou comparáveis) se elas possuírem os mesmos eventos impos- 
síveis, i.e.

$$
\mathbb{P} \sim \mathbb{Q} \Longleftrightarrow[\forall A \in \mathcal{F}, \mathbb{P}(A)=0 \Longleftrightarrow \mathbb{Q}(A)=0]
$$

Uma variável estocástica (v.e.) $X$ assumindo valores em $E$ é uma função mensurável

$$
\begin{aligned}
X: & \Omega \longrightarrow E \\
\omega & \longmapsto X(\omega),
\end{aligned}
$$

sendo que $(\Omega, \mathcal{F}, \mathbb{P})$ é um espaço probabilístico. Um elemento $\omega \in \Omega$ é um cenário de estocasticidade, e, $X(\omega)$ representa uma observação da v.e. se o cenário $\omega$ ocorrer. $X(\omega)$ deve ser entendido como uma realização de $X$ no cenário $\omega$. Se $X$ e $Y$ são duas v.e., então, $X=Y \mathbb{P}$-q.c., se $\mathbb{P}\{\omega \in \Omega, X(\omega)=Y(\omega)\}=1$. A distribuição de $X$ é a medida probabilística em $E$ definida como

$$
\mu_{X}(A)=\mathbb{P}(X \in A) .
$$

Se $\mu_{X}=\mu_{Y}$, então, $X$ e $Y$ são idênticas em probabilidade, i.e., $X \stackrel{d}{=} Y$. Parece razoável dizer que quando $X=Y$-q.c., então, $X \stackrel{d}{=} Y$. Quando $E \subset \mathbb{R}$, tem-se que $X: \Omega \longrightarrow E$ é uma função real. A integral de uma v.e. $X$ em relação a $\mathbb{P}$, que pode ser representada como

$$
\mathbb{E}^{\mathbb{P}}[X]=\int_{E} X(\omega) d \mathbb{P}(\omega)
$$

ou será um número positivo ou $+\infty$. Se $\mathbb{E}^{\mathbb{P}}[X]<\infty$, então, $X$ é $\mathbb{P}$-integrável. Se decompusermos qualquer v.e. $Y$ definida nos reais em suas partes positivas e negativas, i.e., $Y=Y_{+}-Y_{-}$, é possível perceber que, se $\mathbb{E}^{\mathbb{P}}[|Y|]<\infty$, então, $Y_{+}$e $Y_{-}$são integráveis a sua esperança $\mathbb{E}^{\mathbb{P}}[Y]=\mathbb{E}^{\mathbb{P}}\left[Y_{+}\right]-\mathbb{E}^{\mathbb{P}}\left[Y_{-}\right]$será bem comportada. O conjunto de variáveis estocásticas $Y$ (defindas em $\mathbb{R})$, que apresentam a propriedade $\|Y\|_{1}=$ $\mathbb{E}^{\mathbb{P}}[|Y|]<\infty$ são denominadas $\boldsymbol{L}^{1}(\Omega, \mathbb{P})$. Em algumas situações é interessante permitir que variáveis estocásticas assumam valores infinitos, i.e., $E=[0, \infty[\cup\{+\infty\}$, entretanto, se $Y \in \boldsymbol{L}_{1}(\Omega, \mathbb{P})$, então, $Y$ é q.c. finita.

\section{C.2 O Espaço Probabilístico $(\Omega, \mathcal{F}, \mathbb{P})$}

É possível utilizar vários operadores estatísticos, tais como esperança e variância, sem a necessidade de usar espaços probabilísticos. Entretanto, a idéia de espaço probabilístico e v.e. é central para a teoria moderna de probabilidade. Assim, é im- 
portante saber quando estes conceitos são relevantes. Sob a perspectiva de modelagem em finanças, o ponto de partida é o conjunto de observações que assumem valores em $E$, e.g. $E=\mathbb{R}$, para o qual queremos construir um modelo estocástico. É possível representar observações amostrais de uma v.e. $X$, definida no espaço probabilístico $(\Omega, \mathcal{F}, \mathbb{P})$, por $x_{1}, x_{2}, \ldots, x_{n}$. Assim, o único elemento paupável é $E$, que é o conjunto de valores que a v.e. pode assumir, i.e., é a imagem da função $X: \Omega \longrightarrow E$. O conjunto de eventos $\Omega$ não é conhecido previamente. Em algumas circunstâncias, é conveniente relacionar $\Omega$ com $E$, i.e., identificar $\omega \in \Omega$ e observar qual o valor que está sendo atribuído a v.e. $X(\omega)$. O objeto $\omega \in \Omega$ (estocasticidade) atribuirá um determinado valor à v.e. Tem-se que $\Omega$ é denominado estocasticidade ou aleatoriedade e o subconjunto $\omega$ é conhecido por cenário estocástico ou cenário aleatório.

Considere, por exemplo, que os possíveis resultados do experimento estocástico "lançar uma vez um dado e associar a cada evento o valor de face do dado" é a v.e. $X$. Nesta situação é possível identificar, pela definição do experimento, que os possíveis eventos são iguais aos possíveis resultados do experimento: $\Omega:\{1,2,3,4,5,6\}$. No caso de $X(\omega)=\omega$, o resultado da estocasticidade é igual à própria estocasticidade. Nesta situação a escolha de $\Omega$ é dita espaço canônico para a v.e. $X$. No caso em que a v.e. é simplesmente $X(\omega)=\omega$, a medida de probabilidade $\mathbb{P}$ é igual a distribuição de $X$. Neste caso $X$ é um mapeamento de relação um para um com a estocasticidade. Dada uma observação de $X$, é possível identificar qual cenário $\omega$ ocorreu, de tal maneira que, qualquer outra v.e. $Y$ é totalmente determinada por $X$. Assim, ao se construir um espaço canônico é impossível estabelecer qualquer outra variável estocástica no mesmo espaço probabilístico que não seja dependente de $X$. A v.e. $X$, desta maneira, será a única fonte de estocasticidade para todas as outras variáveis estocásticas neste modelo. Como pode ser observado, no caso particular do espaço canônico, que é a forma mais simples de construir um espaço probabilístico para representar uma v.e., a estocasticidade do modelo se confunde com a própria v.e. Em finanças, é interessante distinguir $\Omega$, o conjunto de cenários estocásticos, de $E$, o conjunto de valores da v.e.

No caso da modelagem em finanças, pode-se querer modelar o preço de mercado de ações em uma determinada data futura $T$ como uma v.e. $S_{1}$, sendo que o valor da ação é influenciado por vários fatores, tais como, informação disponível nos meios de comunicação, oferta e demanda de mercado, indicadores econômicos, estabilidade 
política, entre outros. Todos estes fatores podem ser consolidados em uma entidade abstrata denominada $\omega$ (estocasticidade), que pode nem sequer ter uma representação numérica. Ela corresponde ao cenário (futuro) de evolução do mercado. $S_{1}(\omega)$, neste contexto, é o valor da ação caso o cenário de mercado $\omega$ ocorra em $T$. Se a única quantidade de interesse for o valor da ação, então, associa-se o cenário $\omega$ ao valor da ação $S_{1}(\omega)$, que representa o conjunto de cenários que $S_{1}$ assume o mesmo valor. Nesta situação, é possível construir um espaço canônico. Entretanto, ao considerar um modelo mais complexo e mais realístico, nos quais existem diversas ações, tais como $S_{1}, S_{2}, \ldots$, é apropriado distinguir o cenário $\omega$ das variáveis estocáticas $S_{1}(\omega), S_{2}(\omega), \ldots$, sendo que, não necessariamente, assumem valores iguais e, conseqüentemente, torna-se difícil (ou impossível) identificar a fonte de incerteza (estocasticidade) que originou os preços. Neste outro contexto, é possível adicionar quantas variáveis estocásticas forem necessárias, sem ter que modificar o espaço probabilístico incialmente estabelecido para o modelo.

Desta forma, a descrição probabilística de uma v.e. $X$ pode ficar reduzida apenas ao conhecimento de sua distribuição $\mu_{X}$ quando a v.e. $X$ é a única fonte de incerteza (espaço canônico). Em todos as outras situações nos quais houver mais de uma v.e., a medida probabilística $\mathbb{P}$ subjacente à v.e. $X$ possui muito mais informação do que apenas a sua distribuição. A medida probabilística $\mathbb{P}$ conterá toda a informação de dependência entre a v.e. $X$ e as outras variáveis estocásticas do modelo. Logo, $\mathbb{P}$ representa a distribuição conjunta de todas as variáveis estocásticas construídas a partir de $\Omega$, e.g., conhecendo as distribuições $\mu_{X}$ e $\mu_{Y}$ de duas variáveis $X$ e $Y$, não permite conhecer seus comomentos.

\section{C.3 Função Característica}

A função característica de uma v.e. é a transformada de Fourier de sua distribuição. Muitas propriedades de uma v.e. estão relacionadas às propriedades de sua função característica, justificando, a introdução deste conceito no presente estudo. 
Definição C.1 Função Característica A função característica de um vetor de variáveis estocásticas $X$ de dimensão $(d \times 1)$ definida em $\mathbb{R}^{d}$ é uma função $\Phi_{X}$ : $\mathbb{R}^{d} \longrightarrow \mathbb{R}$ definida como

$$
\forall z \in \mathbb{R}^{d}, \Phi_{X}(z)=\mathbb{E}[\exp (i z \cdot X)]=\int_{\mathbb{R}^{d}} \exp (i z \cdot x) d \mu_{X}(x) .
$$

A função característica de uma v.e. determina totalmente a sua distribuição. Duas variáveis estocásticas com a mesma função característica são identicamente distribuídas (i.d.). Uma função característica é sempre contínua e $\Phi_{X}(0)=1$. Adicionalmente, a propriedade de suavidade da função $\Phi_{X}$ depende da existência dos momentos da v.e. $X$. O momento de ordem $n$ de uma v.e. $X$ definida em $\mathbb{R}$ é definido como $m_{n}(X)=$ $\mathbb{E}\left[X^{n}\right]$. Os momentos absolutos de $X$ são definidos como $m_{n}(|X|)=E\left[|X|^{n}\right]$. O momento centrado de ordem $n$ é definido como

$$
\mu_{n}(X)=\mathbb{E}\left[(X-\mathbb{E}[X])^{n}\right], \text { para } n=0,1, \ldots .
$$

Os momentos de uma v.e. podem ou não existir, dependendo de como a distribuição $\mu_{X}$ decai quando $X$ se aproxima de infinito. Um caso interessante é o da distribuição t de Student com $\nu$ g.l., que possui momentos apenas de ordem abaixo de $\nu$, i.e, para que exista no mínimo curtose, é necessário que $\nu>4$ [p.144, Spanos (2000); p.199, Pratt et al. (1996)]. A distribuição exponencial é bem comportada e apresenta todos os momentos finitos para $\alpha>0$. O Quadro 2.1 apresenta algumas funções características de distribuições conhecidas. Os momentos de uma v.e. estão relacionados com a derivada no ponto 0 de sua função característica.

\section{Proposição C.1 Função característica e momento}

1. Se $\mathbb{E}\left[|X|^{n}\right]<\infty$, então, $\Phi_{X}$ possui derivadas contínuas no ponto $z=0$ até ordem $n, e$

$$
\forall k=1,2, \ldots, n, \quad m_{k} \triangleq \mathbb{E}\left[X^{k}\right]=\left.\left[\frac{1}{i^{k}} \frac{\partial^{k}}{\partial z^{k}} \Phi_{X}(z)\right]\right|_{z=0} .
$$

2. Se $\Phi_{X}$ possui derivadas contínuas no ponto $z=0$ até ordem $2 n$, então, $\mathbb{E}\left[|X|^{2 n}\right]<\infty, e$

$$
\forall k=1,2,4 \ldots, 2 n, \quad m_{k} \triangleq \mathbb{E}\left[X^{k}\right]=\left.\left[\frac{1}{i^{k}} \frac{\partial^{k}}{\partial z^{k}} \Phi_{X}(z)\right]\right|_{z=0}
$$


3. $X$ possui momentos finitos de todas as ordens iff $z \longmapsto \Phi_{X}(z)$ é uma função da classe $\mathcal{C}^{\infty}$ no ponto $z=0$. Assim, os momentos de $X$ estão relacionados as derivadas de $\Phi_{X}$ da seguinte forma:

$$
m_{n} \triangleq \mathbb{E}\left[X^{k}\right]=\left.\left[\frac{1}{i^{k}} \frac{\partial^{k}}{\partial z^{k}} \Phi_{X}(z)\right]\right|_{z=0}
$$

Observação Se $\left\{X_{i}\right\}_{i \in \mathbb{N}}$ são variáveis estocásticas independentes, então, a função característica de $S_{n}=\sum_{i=1}^{n} X_{i}$ é o produto das funções características individuais de cada v.e.:

$$
\Phi_{S_{n}}(z)=\prod_{i=1}^{n} \Phi_{X_{i}}(z) .
$$

Outra maneira de entender esta propriedade [p.12, Davidson \& Mackinnon (2003)] é: se $\left\{X_{i}\right\}_{i \in \mathbb{N}}$ são variáveis estocásticas independentes, então, a função densidade conjunta $f\left(x_{1}, \ldots, x_{n}\right)$ pode ser decomposta no produto das funções densidades marginais $f\left(x_{i}\right)$, para $i \in \mathbb{N}$, i.e.,

$$
f\left(x_{1}, \ldots, x_{n}\right)=\prod_{i=1}^{n} f\left(x_{i}\right) .
$$

\section{C.4 Função Geratriz de Momentos}

Define-se uma função geratriz de momentos (m.g.f.) como:

\section{Definição C.2 Função Geratriz de Momentos A função geratriz de momen-} tos de um vetor de variáveis estocásticas definida em $\mathbb{R}^{d}$ é uma função $M_{X}$ definida como

$$
\forall u \in \mathbb{R}^{d}, M_{X}=\mathbb{E}[\exp (u . X)]
$$

Ao contrário da função característica, que é sempre bem definida como uma transformada de Fourier da medida probabilística, a m.g.f. nem sempre é bem definida. A equação (C.11) pode não convergir para certos valores de $u$. Quando $M_{X}$ é bem definida, ela se relaciona formalmente com a função característica $\Phi_{X}$ como segue:

$$
M_{X}(u)=\Phi_{X}(-i u) .
$$


Se a m.g.f. $M_{X}$ de um v.e. $X$ em $\mathbb{R}$ é bem definida na vizinhança $[-\epsilon ; \epsilon]$ do zero (continuidade local), então, nesta situação particular, todos os momentos de $X$ são finitos e podem ser recuperados a partir das derivadas de $M_{X}$, como segue:

$$
m_{n}=\left.\frac{\partial^{n}}{\partial u^{n}} M_{X}(0)\right|_{u=0} \text {. }
$$

\section{C.5 Função Geratriz de Cumulantes}

Seja $X$ uma v.e. e $\Phi_{X}$ sua função característica. Como mencionado anteriormente, $\Phi_{X}(0)=1$ e $\Phi_{X}$ é contínua em $z=0$, tal que $\Phi_{X}(z) \neq 0$ na vizinhança de $z=0$. É possível estabelecer uma versão contínua do $\log$ de $\Phi_{X}$. Assim, existirá uma única função contínua $\Psi_{X}$ definida na vizinhança do ponto zero, i.e.,

$$
\Psi_{X}(0)=0 \quad \text { e } \quad \Psi_{X}(z)=\ln \left[\Phi_{X}(z)\right] .
$$

A função $\Psi_{X}$ é denominada função geratriz de cumulantes (c.g.f.) ou log da função característica de $X$. Cabe ressaltar que, se $\Phi_{X}(z) \neq 0$ para todo $z$, então, a função geratriz de cumulantes pode ser estendida para $\mathbb{R}^{d}$. O cumulante de $X$ é definido como

$$
\kappa_{n}(X)=\left.\left[\frac{1}{i^{n}} \frac{\partial^{n}}{\partial z^{n}} \Psi_{X}(z)\right]\right|_{z=0} .
$$

Aplicando o resultado (C.12), i.e., $z=i u$, e expandindo a função geratriz de cumulantes por Taylor, em torno do ponto $u=0$, tem-se que é possível representar os cumulantes de ordem $n$ por funções polinomiais de momentos $m_{k}(X)$ ou momentos centrados $\mu_{k}(X)$, para $k=0,1,2, \ldots, n$. Os primeiros cumulantes podem ser expressos como:

$$
\begin{aligned}
\kappa_{0}(X) & =m_{0}(X)=\mathbb{E}[X], \\
\kappa_{2}(X) & =\mu_{2}(X)=m_{2}(X)-m_{1}(X)^{2}=\mathbb{V}[X], \\
\kappa_{3}(X) & =\mu_{3}(X)=m_{3}(X)-3 m_{2}(X) m_{1}(X)+2 m_{1}(X)^{3}, \\
\kappa_{4}(X) & =\mu_{4}(X)-3 \mu_{2}^{2}(X) .
\end{aligned}
$$

Versões de cumulantes de escalas padronizadas podem ser obtidas ao normalizar $\kappa_{n}$ pelo desvio padrão elevado à potência $n$-ésima:

$$
\alpha_{3}(X)=\frac{\kappa_{3}(X)}{\kappa_{2}^{3 / 2}(X)}, \quad \alpha_{4}(X)=\frac{\kappa_{4}(X)}{\kappa_{2}^{2}(X)} .
$$


A quantidade $\alpha_{3}(X)$ é conhecida como coeficiente de assimetria de $X$. Se $\alpha_{3}(X)>0$, então, $X$ possui assimetria positiva. A quantidade $\alpha_{4}(X)$ é conhecido como excesso de curtose de $X$. A v.e. $X$ é leptocúrtica se $\alpha_{4}(X)>0$. Cabe ressaltar que, se $X$ possui distribuição gaussiana, $\Psi_{X}=-\frac{1}{2}\left[\ln (2 \pi \sigma)+\left(\frac{x-\gamma}{\sigma}\right)^{2}\right]$ é um polinômio de segundo grau, tal que, $\forall n \geq 3, \kappa_{n}(X)=0$. Assim, é possível interpretar $\alpha_{3}(X)$, $\alpha_{4}(X)$ e outros cumulantes padronizados de ordem superior, como medidas de desvios da distribuição gaussiana. Por construção, os coeficientes de assimetria e a curtose são invariantes às mudanças de escala:

$$
\forall \lambda>0, \quad \alpha_{3}(\lambda X)=\alpha_{3}(X) \quad \text { e } \quad \alpha_{4}(\lambda X)=\alpha_{4}(X) .
$$

A função geratriz de cumulantes de variáveis independentes podem ser somadas quando as variáveis são somadas. Esta propriedade é facilmente obtida através do resultado (C.9). Se $\left\{X_{i}\right\}_{i=1,2, \ldots n}$ são variáveis estocáticas independentes, então,

$$
\Psi_{X_{i}+X_{2}+\ldots+X_{n}}(z)=\sum_{i=1}^{n} \Psi_{X_{i}}(z) .
$$




\section{Apêndice D \\ Convergência de Variáveis Estocásticas}

Ao considerar seqüências ou famílias de variáveis estocásticas, é possível obter alguns tipos diferentes de convergência. As famílias de distribuições apresentadas nas definições a seguir estão indexadas por números inteiros. Apesar disso, elas também são aplicadas à indexadores contínuos, i.e., $\mathbb{R}$.

\section{D.1 Convergência Quase-Certa}

Considere uma seqüência de variáveis estocásticas $\left(X_{n}\right)_{n \geq 1}$ que assume valores em um espaço vetorial normalizado $E$, i.e., $E=\mathbb{R}^{d}$. Dado que uma v.e. é uma função $X: \Omega \longrightarrow E$ da estocasticidade $\omega \in \Omega$, existem vários tipos de convergência de uma seqüência de variáveis estocásticas. O conceito mais simples de convergência de funções é a convergência pontual, no qual para cada $\omega \in \Omega$, a seqüência $\left\{X_{n}(\omega)\right\}_{n \geq 1}$ converge para $X(\omega) \in E$. Este conceito é forte em diversas situações, já que exige convergência para qualquer cenário estocástico $\omega \in \Omega$, inclusive para os cenários impossíveis, i.e., de probabilidade zero. É importante, desta maneira, introduzir ao estudo, o conceito de convergência quase-certa (q.c.), que considera a convergência pontual apenas para os cenários estocásticos possíveis (probabilidade maior que zero).

Definição D.1 Convergência quase-certa A seqüência $\left\{X_{n}\right\}_{n \geq 1}$ de variáveis estocásticas definidas em $(\Omega, \mathcal{F}, \mathbb{P})$ converge quase-certamente para uma v.e. $X$ se

$$
\mathbb{P}\left(\lim _{n \rightarrow \infty} X_{n}=X\right)=1 .
$$

Esta definição faz sentido para as variáveis $\left\{X_{n}\right\}_{n \geq 1}$, quando estas estão definidas no mesmo espaço probabilístico $(\Omega, \mathcal{F}, \mathbb{P})$. Cabe ressaltar que, a convergência quasecerta não implica convergência de momentos, i.e., os momentos da v.e. $X$ resultante da convergência não serão necessariamente finitos. Se $X_{n} \longrightarrow X$ converge quase- 
certamente, então, os momentos $\mathbb{E}\left[X_{n}^{k}\right]<\infty$ são bem definidos para $1 \leq n<\infty$. Entretanto, o limite $\lim _{n \rightarrow \infty} \mathbb{E}\left[X_{n}^{k}\right]=\mathbb{E}\left[X^{k}\right]$ pode não ser uma quantidade finita.

\section{D.2 Convergência em Probabilidade}

Enquanto a convergência quase-certa de $\left\{X_{n}\right\}_{n \geq 1}$ considera o comportamento do processo dado um cenário estocástico $\left\{X_{n}(\omega)\right\}_{n \geq 1}$, o conceito de convergência em probabilidade apenas estabelece a probabilidade do evento, quando $n \longrightarrow \infty$.

Definição D.2 Convergência em probabilidade Uma seqüência $\left\{X_{n}\right\}_{n \geq 1}$ de variáveis estocástiscas em $(\Omega, \mathcal{F}, \mathbb{P})$ converge em probabilidade para um v.e. $X$, se para qualquer $\epsilon>0$

$$
\lim _{n \rightarrow \infty} \mathbb{P}\left(\left|X_{n}-X\right|>\epsilon\right)=0
$$

A convergência em probabilidade também é denominada como

$$
X_{n} \underset{n \rightarrow \infty}{\stackrel{\mathbb{P}}{\longrightarrow}} X
$$

Convergência quase-certa implica convergência em probabilidade, mas o contrário não é verdadeiro. Cabe ressaltar que, convergência em probabilidade também exige que as variáveis estocásticas sejam definidas no mesmo espaço probabilístico $(\Omega, \mathcal{F}, \mathbb{P})$.

\section{D.3 Convergência em Distribuição}

Em algumas situações, o valor que a v.e. $X$ assume não é observadado. O que se observa é a esperança de funções que dependam desta v.e., i.e., $\mathbb{E}[f(X)]$. Estas esperanças são equivalentes para variáveis estocásticas que possuam a mesma distribuição. Assim, $\mathbb{E}\left[f\left(X_{n}\right)\right]$ converge para $\mathbb{E}[f(X)]$ para um determinado conjunto de funções $f: E \longrightarrow \mathbb{R}$. Se o conjunto funções for realmente grande, então, deve existir uma única distribuição para $X$. Um conjunto adequado de funções é o conjunto das funções contínuas contornadas $f: E \longrightarrow \mathbb{R}$., que são representados por $\mathcal{C}^{b}(E, \mathbb{R})$. 
Definição D.3 Convergência em distribuição A seqüência $\left\{X_{n}\right\}$ de variáveis estocásticas defnidas em E converge em distribuição de uma v.e. X se, para qualquer função contínua contornada $f: E \longrightarrow \mathbb{R}$

$$
\mathbb{E}\left[f\left(X_{n}\right)\right] \underset{n \rightarrow \infty}{\stackrel{d}{\longrightarrow}} \mathbb{E}[f(X)] .
$$

Ao representar por $\mu_{n}$ a distribuição de $X_{n}$, onde $\mu_{n}$ é uma medida probabilística em $E$, a equação (D.4) é equivalente a

$$
\forall f \in \mathcal{C}^{b}(E, \mathbb{R}), \quad \int_{E} d \mu_{n}(x) f(x) \underset{n \rightarrow \infty}{\stackrel{d}{\longrightarrow}} \int_{E} d \mu(x) f(x) .
$$

Ao contrário da convergência quase-certa, é possível perceber que a definição de convergência em distribuição, ao contrário da convergência quase-certa, não depende da variáveis estocástica, mas apenas de sua distribuição. A convergência em distribuição de $\left\{X_{n}\right\}_{n \geq 1}$ também é denominada convergência fraca da medidas $\left\{\mu_{n}\right\}_{n \geq 1}$ em $E$. Assim,

$$
\mu_{n} \underset{n \rightarrow \infty}{\stackrel{d}{\rightleftharpoons}} \mu \quad \text { ou } \quad X_{n} \underset{n \rightarrow \infty}{\stackrel{d}{\longrightarrow}} X .
$$

Uma importante característica da convergência em distribuição é que, ao contrário das outras formas de convergência, não é necessário que as variáveis estocásticas sejam definidas no mesmo espaço probabilístico. Entretanto, nos casos nos quais as variáveis estocásticas são definidas no mesmo espaço probabilístico, convergência em distribuição implica convergência em probabilidade:

Proposição D.1 Se $\left\{X_{n}\right\}$ converge em distribuição para $X$, então, para qualquer conjunto A com contorno $\partial A$,

$$
\mu(\partial A)=0 \Longrightarrow\left[\mathbb{P}\left(X_{n} \in A\right)=\mu_{n}(A) \underset{n \rightarrow \infty}{\stackrel{d}{\longrightarrow}} \mathbb{P}(X \in A)=\mu(A)\right]
$$

Cabe ressaltar que, diferentemente das outras formas de convergência, a convergência em distribuição não é estável em operações de soma, i.e., se $\left\{X_{n}\right\}$ e $\left\{Y_{n}\right\}$ convergem em distribuição para $X$ e $Y$, então, não é verdade que $\left\{X_{n}+Y_{n}\right\}$ converge em distribuição para $(X+Y)$. Entretanto, pela própria Definição D.3, se $X_{n} \stackrel{d}{\longrightarrow} X$, então, para qualquer função contínua $g$, então, $g\left(X_{n}\right) \stackrel{d}{\longrightarrow} g(X)$. 
A idéia de convergência fraca é relevante para o estudo de aproximações numéricas originadas através de discretizações de modelos contínuos. Na modelagem em finanças, por exemplo, o preço de opções pode ser expresso como a esperança dos fluxos de caixas futuros na medida neutralizadora do risco. A convergência fraca do processo do preço do ativo-objeto discretizado no tempo implica que o preço da opção no modelo discreto convergirá para o preço obtido no modelo contínuo.

Também é possível relacionar a convergência em distribuição com a convergência pontual de funções características, como segue:

Proposição D.2 $\left\{X_{n}\right\}_{n \geq 1}$ converge em distribuição para $X$, iff para qualquer $z \in \mathbb{R}^{d}$

$$
\Phi_{X_{n}}(z) \longrightarrow \Phi_{X}(z)
$$

Cabe ressaltar que, a convergência pontual de $\left\{\Phi_{X_{n}}\right\}$ não implica existência de um limite para $\left\{X_{n}\right\}$, pois o limite pontual de $\left\{\Phi_{X_{n}}\right\}$ não é necessariamente uma função característica. Convergência em distribuição não necessariamente garante convergência de momentos, pois não é possível assumir que $f$ é uma função polinomial, pois funções polinomiais não são definidas em intervalos fechados. Na verdade, os momentos de $X_{n}$ e $X$ podem nem existir. 


\section{Apêndice E \\ Funções Bessel Modificadas}

As funções Bessel modificadas são funções Bessel de argumentos imaginários. Elas ssoão funções especiais e, por já terem sido bastantes estudadas já existem algoritimos rápidos para serem calculadas e implementadas [Press (2002) et al., p.241-256]. Considere a seguinte equação:

$$
z^{2} \frac{d^{2} w}{d z^{2}}+z \frac{d w}{d z}-\left(z^{2}+\nu^{2}\right) w=0 .
$$

A função Bessel modificada do primeiro tipo $I_{\nu}(z)$ para $z \geq 0$ e $\nu \geq 0$ é igual a solução para equação diferencial E.1, que possui limite finito para $z \longrightarrow 0$. A função Bessel modificada de segundo tipo $K_{\nu}(z)$ para $z \geq 0$ e $\nu \geq 0$ é igual a solução para a equação E.1 que possui limite finito para $z \longrightarrow \infty$. Estas funções são sempre linearmente independentes e adimitem a seguinte série de expansão:

$$
\begin{aligned}
I_{\nu}(z) & =\sum_{k=0}^{\infty} \frac{(z / 2)^{\nu+k}}{k ! \Gamma[k+\nu+1]}, \quad \nu \notin\left\{\mathbb{Z}_{-} \backslash\{0\}\right\}, \\
I_{-n}(z) & =I_{n}(z), \quad n \in \mathbb{N}, \\
K_{\nu}(z) & =\frac{\pi}{2} \frac{I_{-\nu}(z)-I_{\nu}(z)}{\sin (\pi \nu)} .
\end{aligned}
$$

Para valores inteiros de $\nu$ a última expressão pode ser entendida para casos limites. Para $\nu \in \mathbb{Z}$, tem-se que $K_{-\nu}(z)=K_{\nu}(z)$.

\section{E.1 Comportamento assintótico}

Para $z \longrightarrow+\infty$ tem-se

$$
\begin{aligned}
I_{\nu}(z) & =\frac{\exp \{z\}}{\sqrt{2 \pi z}}\left[1+O\left(\frac{1}{z}\right)\right], \\
K_{\nu}(z) & =\exp \{-z\} \sqrt{\frac{\pi}{2 z}}\left[1+O\left(\frac{1}{z}\right)\right] .
\end{aligned}
$$


Para $z \longrightarrow 0$ tem-se

$$
\begin{aligned}
I_{\nu}(z) & \sim \frac{(z / 2)^{\nu}}{\Gamma[\nu+1]}, \quad \nu \notin\left\{\mathbb{Z}_{-} \backslash\{0\}\right\}, \\
K_{0}(z) & \sim-\ln z, \\
K_{\nu}(z) & \sim \frac{1}{2} \Gamma[\nu](z / 2)^{-\nu}, \quad \nu>0 .
\end{aligned}
$$

\section{E.2 Expressões analíticas de funções Bessel modificadas definidas nos naturais}

$$
\begin{aligned}
I_{n-\frac{1}{2}}(z) & =\sqrt{\frac{2}{\pi z}} z^{n}\left(\frac{1}{z} \frac{d}{d z}\right)^{n} \cosh z, \quad n \in \mathbb{N}, \\
K_{n-\frac{1}{2}}(z) & =\sqrt{\frac{\pi}{2 z}} z^{n}\left(-\frac{1}{z} \frac{d}{d z}\right)^{n} \exp \{-z\}, \quad n \in \mathbb{N},
\end{aligned}
$$

sendo que $\cosh y \triangleq \frac{1}{2}(\exp \{y\}+\exp \{-y\})$ é denominado coseno hiperbólico.

\section{E.3 Representação por integral}

Representação por integral de Poisson:

$$
\begin{aligned}
I_{\nu}(z) & =\frac{(z / 2)^{\nu}}{\sqrt{\pi} \Gamma\left[\nu+\frac{1}{2}\right]} \int_{-1}^{1}\left(1-t^{2}\right)^{\nu-1 / 2} \cosh (z t) d t \\
K_{\nu}(z) & =\frac{\exp \{-z\}}{\Gamma\left[\nu+\frac{1}{2}\right]} \sqrt{\frac{\pi}{2 z}} \int_{0}^{\infty} \exp \{-t\} t^{\nu-1 / 2}\left(1+\frac{t}{2 z}\right)^{\nu-1 / 2} d t .
\end{aligned}
$$

A representação de $K_{\nu}$ por meio da integral de Sommerfeld é:

$$
\begin{aligned}
K_{\nu}(z) & =\frac{1}{2} \int_{-\infty}^{\infty} \exp (-z \cosh \psi+\nu \psi) d \psi \\
& =\frac{1}{2}\left(\frac{z}{2}\right)^{\nu} \int_{0}^{\infty} \exp \left(-t-\frac{z^{2}}{4 t}\right) t^{-\nu-1} d t .
\end{aligned}
$$

Esta representação permite calcular a integral da seguinte maneira:

$$
\int_{0}^{\infty} \exp \left(-\frac{\alpha^{2} t}{2}-\frac{\beta^{2}}{2 t}\right) \frac{d t}{t^{+\nu+1}}=2\left(\frac{\alpha}{\beta}\right)^{\nu} K_{\nu}(\alpha \beta) .
$$




\section{E.4 Relação entre as funções Bessel e o argumento real}

A função Bessel modificada do primeiro tipo pode ser representada por meio da função Bessel do primeiro tipo com argumento imaginário:

$$
I_{\nu}(z)=\exp \{-i \pi \nu / 2\} J_{\nu}(i z),
$$

e a função Bessel modificada de segundo tipo pode ser representada por meio de função Hankel do primeiro tipo:

$$
K_{\nu}(z)=\frac{\pi}{2} \exp \{i \pi(\nu+1) / 2\} H_{\nu}^{(1)}(i z) .
$$

